This document is downloaded from DR-NTU (https://dr.ntu.edu.sg) Nanyang Technological University, Singapore.

Design and implementation of variable digital filters with low computational complexity

Xu, Wei Jing

2014

https://hdl.handle.net/10356/61719

https://doi.org/10.32657/10356/61719 


\title{
Design and Implementation of Variable Digital Filters with Low Computational Complexity
}

$\underline{\text { Xu Wei Jing }}$

School of Electrical \& Electronic Engineering

\author{
A thesis submitted to the Nanyang Technological University \\ in partial fulfillment of the requirement for the degree of \\ Doctor of Philosophy
}




\section{Acknowledgments}

This research work was carried out at Temasek Laboratory of Nanyang Technological University during the years 2008 - 2013. I am deeply grateful to my supervisor, Prof. Yu Ya Jun, for her constant encouragement and inspiring suggestions during all phases of my work so that I can overcome the difficulties encountered in my research and become a capable researcher. Besides, her persistence on research work of high standard influences me profoundly on my attitude towards research work as well as my life.

I am also grateful to Dr. Huang Chaogeng, Dr. Shi Dong, Dr. Lee Jun Wei, Dr. Yang Chun Zhu, Mr. Leong Jenn Gan, Mr. Zhu Zhi Dong, Miss Xu Sai Hua, Mr. Shen Tian and other people I have met in Temasek Lab, for a pleasant and inspiring atmosphere. At Temasek Lab, I have had many interesting discussions with these experienced scientists. Besides, I wish to thank the visiting scholars Prof. Tian-Bo Deng (Toho University) with whom I had fruitful discussions on Lagrange interpolations, Dr. Oscar Gustafsson (Linköping University) for his kind help in the practical issues, and Dr. Huang Chao Geng for his help solving latex issues.

Finally, my warmest thanks go to my parents, who have constantly encouraged me to study as much as possible, and supported me to live through the toughest period. 


\section{Summary}

Variable digital filters (VDFs) have various applications in digital communications, sampling rate conversion, array signal processing, etc. The useful variable characteristics of digital filters include variable bandedges and variable fractional delays (VFDs). However, the computational complexity of VDFs is usually higher than that of the filters with fixed characteristics. The objective of this research is to investigate the design and implementation of VDFs with low computational complexity. Same as the fixed filter design, there are closed-form approaches and numerical optimization techniques available for the design of VDFs. In this thesis, both the closed-from approach and numerical optimization techniques are studied.

In the closed-form design, the Lagrange interpolation, implemented in the Farrow structure, is one of the popular approaches for the design of VFD filters and the researches are focused on the reduction of the computational complexity by using matrix transformations. In this thesis, the dependance between the subfilter coefficients of the Lagrange interpolation is investigated and discovered. An efficient implementation structure making use of the coefficient dependance was proposed to significantly reduce the computational complexity.

While the closed-form VFD filters are convenient to design, it can achieve VFD only for a narrow frequency range. When wider variation range and more accurate control on the variable characteristics are required, numerical optimization techniques are generally adopted. In numerical optimizations, an objective function is minimized subject to some constraints. In the existing researches, the objective functions to be minimized include 
frequency response error, phase error, phase delay error and group delay error. However, in the most common applications of the VFDs, the time domain instantaneous delayed samples are estimated. In this thesis, for such applications, the relations between the above frequency domain errors (in particular the frequency response error, phase error and group delay error), and the time domain errors are investigated. The design criteria of the VFD filters for this particular application are identified.

Besides the investigation on the design criteria, a new VFD filter design approach is proposed. In this approach, the full band input signal is split into several subbands by a newly proposed filter bank. By shifting each subband a proper phase, the VFD is realized by combining necessary subbands. The proposal VFD technique can be incorporated with the variable bandedge characteristic with little extra complexity. Hence, the filters with simultaneously variable bandedges and fractional delays (VBFDs) are obtained. In the design of the VBFD filters, the split subbands can be either kept or discarded to form the variable bandedges. However, this requires the bandwidth of the individual band of the filter bank to be narrower than the transition bandwidth of the VBFD filters. By introducing a shaping filter to the last retained band to form the transition band of the VBFD filters, the bandwidth of the individual band of the filter bank may be relaxed to about twice of the transition bandwidth of the VBFD filters. Compared with the existing VBFD filter design techniques, the proposed approaches significantly reduce the computational complexity of the VBFD filters. 


\section{Contents}

Acknowledgments $\quad$ i

Summary ii

List of Figures $\quad$ viii

List of Tables $\quad$ xiii

List of Abbreviations $\quad$ xv

1 Introduction 1

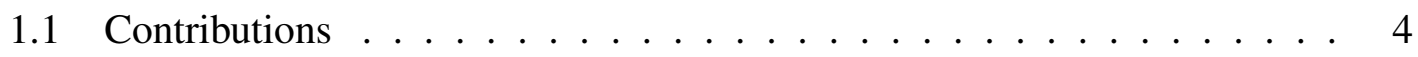

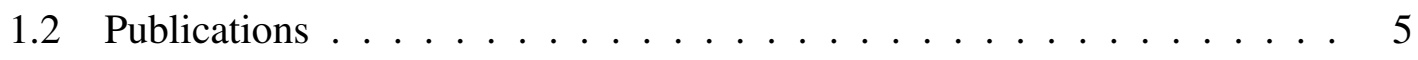

1.2.1 Journal Papers . . . . . . . . . . . . . . . 5

1.2.2 Conference Papers ................ 5

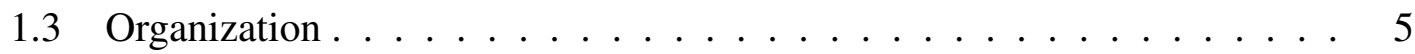

2 Basic concepts and literature reviews $\quad 7$

2.1 Variable Bandedge Filters . . . . . . . . . . . . . . . . 7

2.2 Design of Variable Bandedge Filters . . . . . . . . . . . . . . . . 8

2.3 Fractional Delay Filters . . . . . . . . . . . . . . . 9

2.3.1 Phase Shifts and Time Domain Sample Delays . . . . . . . . . 9

2.3.2 Phase Delay and Group Delay . . . . . . . . . . . . . 11

2.3.3 Fractional Delay .................. 13

2.3.4 Fractional Delay Filters . . . . . . . . . . . . . . . . . 14

2.3.5 Applications of Fractional Delays . . . . . . . . . . . . . . 17 
2.4 Variable Fractional Delay Filters . . . . . . . . . . . . . . . . 19

2.5 Design of Variable Fractional Delay Filters . . . . . . . . . . . . 21

2.5.1 Closed-form Approaches . . . . . . . . . . . . . 23

2.5.2 Optimization Approaches ................. 24

2.6 Variable Bandedge and Fractional Delay Filters . . . . . . . . . . . 26

2.7 Design of Variable Bandedge and Fractional Delay Filters . . . . . . . . . 29

2.8 Frequency Response Masking and Fast Filter Bank . . . . . . . . . . . 31

2.8.1 Frequency Response Masking . . . . . . . . . . . . . . . . 32

2.8.2 Fast Filter Bank . . . . . . . . . . . . . . . . . . . . 34

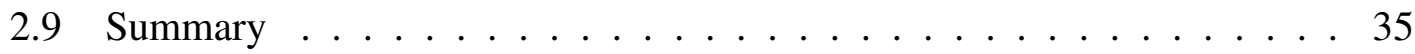

3 Polynomial Implementation of Lagrange-type Variable Fractional Delay Fil$\begin{array}{ll}\text { ters } & 37\end{array}$

3.1 The Farrow Structure Based Lagrange-Type Variable Fractional Delay

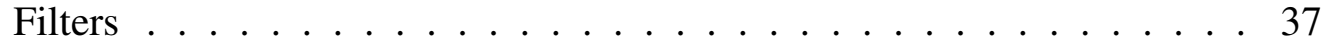

3.2 Properties of the Transformed Coefficient Matrix . . . . . . . . . . . 40

3.3 Polynomial Implementation Strategy . . . . . . . . . . . . . . . . . . 44

3.3.1 Polynomial Implementation for even order Lagrange-type VFD

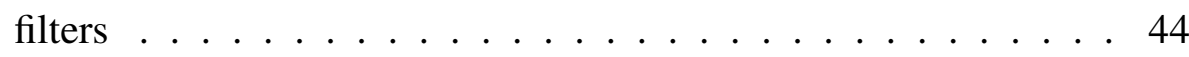

3.3.2 Polynomial Implementation for odd order Lagrange-type VFD

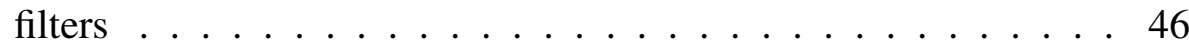

3.4 Numerical Examples . . . . . . . . . . . . . . . . . 48

3.4.1 Implementation of a 5th order Lagrange-type VFD filter . . . . . 48

3.4.2 Design of a 6 th order Lagrange-type VFD filter . . . . . . . . 51

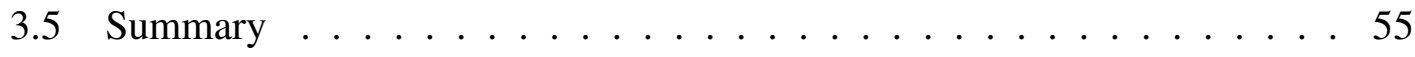

4 Investigation on the Optimization Criteria for the Design of VFD Filters 57

4.1 Review on Design Criteria of VFD Filters . . . . . . . . . . . 58

4.2 Theoretical Analysis ........................ 59 
4.3 Verification by Numerical Examples ............... 63

4.3.1 Single Frequency Sinusoid Signal . . . . . . . . . . . . 63

4.3.2 White Noise Signal . . . . . . . . . . . . . . 65

4.3 .3 Chirp Signal ...................... 68

4.3.4 Real World Signal ... . . . . . . . . . . 72

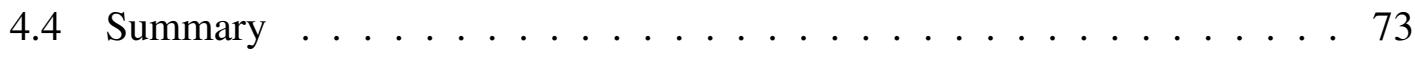

5 Fast Filter Bank Approach for the Design of Variable Fractional Delay Filters $\quad 75$

5.1 Proposed Mixed-radix Fast Filter Bank . . . . . . . . . . . . . 76

5.1.1 Problem Formulation . . . . . . . . . . . . . 77

5.1.2 Design of Filter Banks . . . . . . . . . . . . 78

5.1 .3 Implementation Issue . . . . . . . . . . . . . . 82

5.1 .4 Computational Complexity . . . . . . . . . . . . 84

5.2 Optimization Design of Prototype Filter $G_{0}(z)$ in FFB $\quad \ldots \ldots$. . . . 85

5.3 Design Parameters . . . . . . . . . . . . . . . . 89

5.3.1 Determination of the stage number of the FFB . . . . . . 89

5.3.2 Determination of $\delta$ for the estimation of the Prototype Filter orders 89

5.3.3 Summary of Design Steps . . . . . . . . . . . . 93

5.4 Design Example . . . . . . . . . . . . . . . . 93

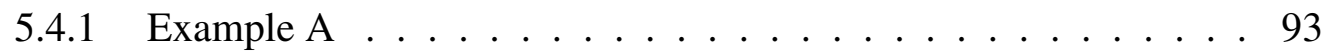

5.4 .2 Example B ........................ 95

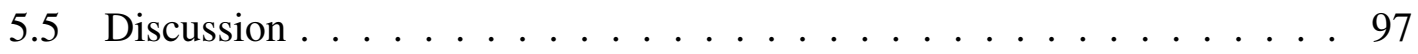

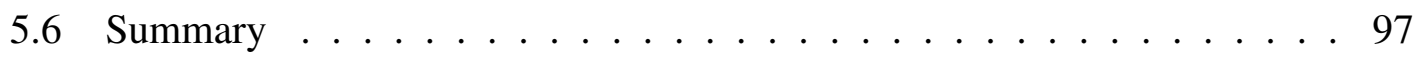

6 Fast Filter Bank Approach for the Design of Variable Bandedge and Fractional Delay Filters $\quad 99$

6.1 Approach 1: Band Combination for the Synthesis of VBFD . . . . . . . 100

6.2 Approach 2: Band Shaping for the Synthesis of VBFD . . . . . . . . . 104 
6.2.1 Construction of Shaping Filters . . . . . . . . . . . 105

6.2.2 Sharing of the Shaping Filters . . . . . . . . . . . 107

6.3 Complexity Analysis . . . . . . . . . . . . . . . . 110

6.3.1 Computational Complexity . . . . . . . . . . . 112

6.3.2 Implementation Complexity . . . . . . . . . . . . 113

6.3.3 Switch Complexity . . . . . . . . . . . . . . 114

6.4 Design Parameters . . . . . . . . . . . . . . . 115

6.4.1 Determination of the Number of the FFB Stages . . . . . . 115

6.4.2 Adoption of the Shaping Filters . . . . . . . . . . . . 116

6.4.3 Summary of Design Steps . . . . . . . . . . . . 116

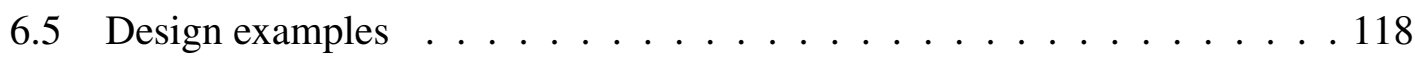

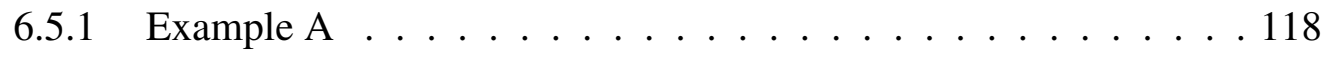

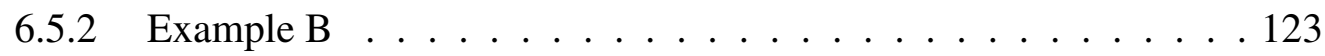

6.5.3 Example $\mathrm{C} \ldots \ldots . \ldots . \ldots . \ldots . \ldots \ldots$

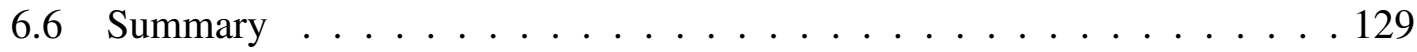

7 Conclusion and Future Work 133

7.1 Research Work Conclusion . . . . . . . . . . . . . 133

7.2 Future Work Briefing . . . . . . . . . . . . . . . . 134

$\begin{array}{ll}\text { References } & 139\end{array}$ 


\section{List of Figures}

2.1 The specifications of a lowpass filter with variable bandedges. . . . . . 8

2.2 The relation between time domain sample delay and phase shifts. . . . . . 10

2.3 Plots of signal $x_{n}$ (solid line), its envelop $x_{e}$ (dash-dot line), $y_{n}$ (dot line) and the envelop $y_{e}$ (dash line). . . . . . . . . . . . 12

2.4 The concept of fractional delay. . . . . . . . . . . . . . . . 13

2.5 Impulse responses $\hat{h}(n)$ of an ideal delay filter with a delay (a) $D=1$ and (b) $D=1.3$, respectively. . . . . . . . . . . . . 16

2.6 Fractional delay in beamforming . . . . . . . . . . . . . 17

2.7 Fractional delay in sampling rate conversion . . . . . . . . . . 18

2.8 Block diagram of an incommensurate-ratio sampling-rate converter . . . . 18

2.9 The specifications of a VFD filter. . . . . . . . . . . 20

2.10 The Farrow structure. . . . . . . . . . . . . . . . 22

2.11 Specifications for (a) magnitude response and (b) phase response of a lowpass filter with variable passband edge $\phi$ and variable fractional delay $d .28$

2.12 The parallel implementation of VBFD filters. . . . . . . . . . 30

2.13 The nested Farrow structure for the implementation of VBFD filters . . . 31

2.14 Implementation structure for VBFD filters proposed in [91] . . . . . . . . 32

2.15 The structure of the frequency response masking filter. . . . . . . . 33

2.16 The frequency responses of FRM sub-filters. . . . . . . . . . . . 33

2.17 The structure of a 3 -stage FFB. . . . . . . . . . . . . . . 34

2.18 The frequency responses of the filters in an 8-channel FFB. . . . . . . 36 
3.1 The even-odd structure for the Lagrange interpolation for (a) even $N$ and (b) odd $N$, respectively. . . . . . . . . . . . . . . . . . . 40

3.2 Polynomial structure for even $N$ th order Lagrange-type VFD filters. . . . 46

3.3 Polynomial structure for odd $N$ th order Lagrange-type VFD filters. . . . . 47

3.4 The implementation structure of the polynomials (in dash box) as well as the 5 th order Lagrange-type VFD filter. . . . . . . . . . . . . . . . 49

3.5 Example A: the (a) frequency response errors (b) magnitude errors and (c) phase errors of the 5th order Lagrange-type VFD filters implemented by the proposed technique and the technique in [34]. . . . . . . . 50

3.6 Polynomial structure for the 6th order $(N=6)$ Lagrange-type VFD filters. 52

3.7 MCM techniques applied to the constant matrix multiplication. . . . . . . 53

3.8 Example B: the (a) frequency response errors, (b) magnitude errors and (c) phase errors of the 6th order Lagrange-type VFD filters implemented by the proposed technique and the technique in [34]. . . . . . . . . 54

4.1 The time domain approximation system, where $(.)^{2}$ denotes the square

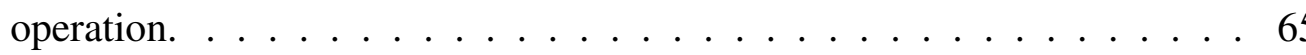

4.2 Frequency spectrum of signal segments of a chirp signal $x_{n}$ given in (4.19) for (a) $n=0, \ldots, 999$, (b) $n=1000, \ldots, 1999$, (c) $n=2000, \ldots, 2999$ and (d) $n=3000, \ldots, 3999 \ldots \ldots \ldots$

4.3 The frequency spectrum of the data samples of the music for test. . . . . . 74

5.1 The conceptual diagram of the filter bank approach for the design of VFD filters, where $\theta=\frac{2 \pi}{N} d . \ldots \ldots \ldots$. . . . . . . . . . . . . . . . .

5.2 Illustration of a filter bank with uniform bands of triangular frequency

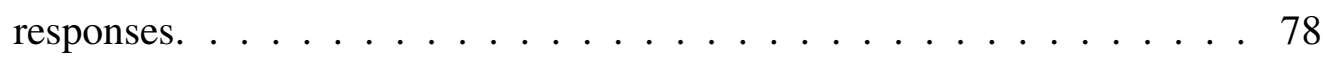

5.3 The structure of a 3 -stage mixed-radix $(3 / 2)$ FFB. . . . . . . . . . . . 79 
5.4 The frequency response of (a) the prototype filter $G_{0}\left(e^{j \omega}\right)$, and its compressed and shifted versions of (b) $G_{0}\left(e^{j 2^{R-1} \omega}\right)$, (c) $G_{0}\left(e^{j\left(2^{R-1} \omega-\frac{2 \pi}{N}\right)}\right)$, and (d) $G_{0}\left(e^{j\left(2^{R-1} \omega-\frac{4 \pi}{N}\right)}\right)$. The frequency response of (e) $G_{1,0}^{a}\left(e^{j \omega}\right)$ and (f) $G_{2,0}^{a}\left(e^{j \omega}\right) . \quad N=3 \times 2^{R-1}$ for an $R$-stage mixed-radix (3/2) FFB. In this example, $R=3 . \ldots \ldots \ldots$. . . . . . . . . . . 80

5.5 The refined implementation structure for VFD filter, where $\tilde{X}_{n}\left(e^{j \omega}\right)$ represents $X_{n}\left(e^{j \omega}\right)$ or $X_{-n}^{*}\left(e^{j \omega}\right)$ for $n=1, \ldots, \frac{N}{2}-1 \ldots \ldots . \ldots 3$

5.6 The maximum frequency response errors vs. the number of stages of the FFB $R$ and the passband edge $\omega_{p} \ldots \ldots \ldots$. . . . . . . . . . 90

5.7 The principle of the synthesis of VFD using filter bank. (a) the frequency responses of two adjacent bands $k-1$ and $k$, (b) the vector representations of the superposition of the phase shifted signals and (c) the error effect. . 91

5.8 Example A: The frequency response errors (Left column), magnitude errors (Middle column) and phase errors (Right column) of the designed VFD filter for $d=0, \pm 0.1, \ldots, \pm 0.5$ (rows from top to bottom). . . . . . 94

5.9 The frequency response (Left column), magnitude (Middle column) and phase errors (Right column) of the VFD filter designed by the proposed technique for $d=0, \pm 0.1, \ldots, \pm 0.5$ (rows from top to bottom). . . . . . 96

6.1 The structure of an $R$-stage $N$-band mixed-radix (3/2) FFB to synthesize the VBFD filter. . . . . . . . . . . . . . . . . 102

6.2 Structure of the variable filter. (a) Overall structure; (b) an " $\mathrm{A}+\mathrm{S}$ " unit; (c) and (d) the switch status of the "A+S" unit for different bands, where $\tilde{X}_{n}\left(e^{j \omega}\right)$ represents $X_{n}\left(e^{j \omega}\right)$ or $X_{-n}^{*}\left(e^{j \omega}\right)$ for $n=1, \ldots, \frac{N}{2}-1 . \ldots 103$

6.3 The structure of an $R$-stage $N$-band mixed-radix (3/2) FFB with a shaping filter $F_{k}\left(e^{j \omega}\right)$ for band $k \ldots \ldots \ldots$. . . . . . . . . . . . . . . . 
6.4 Block diagram of the synthesis of the VBFD filter with a configurable shaping structure. . . . . . . . . . . . . . . . 106

6.5 The configurable shaping structure to be cascaded to each band. . . . . . 107

6.6 The implementation of the delay and shaping filter of a shaping structure in Figure 6.4. . . . . . . . . . . . . . . . . . . 107

6.7 Frequency responses of bandpass shaping filters for bands $k$ and $k+3$. . 109

6.8 The frequency responses of (a) the prototype filter (b) $B_{0}\left(e^{j \omega}\right)$ (c) $B_{1}\left(e^{j \omega}\right)$ (d) $B_{2}\left(e^{j \omega}\right) \ldots \ldots \ldots \ldots \ldots \ldots$

6.9 The structure of (a) an $R$-stage $N$-band mixed-radix FFB with the technique of shaping filter sharing and $(b)(c)(d)$ the multiplier blocks of the 3 shaping filters, respectively. . . . . . . . . . . . . . . 111

6.10 The maximum (a) magnitude and (b) phase errors vs. $\Delta \omega$ for $R=3,4,5$

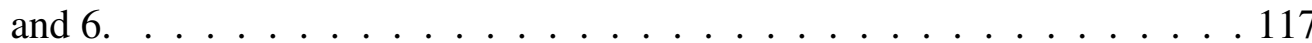

6.11 Example A: the maximum magnitude errors vs. $d$ and $\phi$, of the VBFD filters designed by (a) the Proposed ${ }_{1}$ (b) the Proposed $_{2}$ (c) the technique

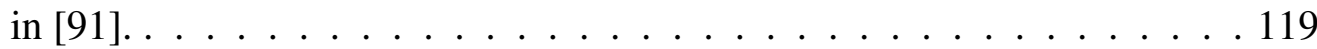

6.12 Example A: the maximum phase errors vs. $d$ and $\phi$, of the VBFD filters designed by (a) the Proposed $_{1}$ (b) the Proposed $_{2}$ (c) the technique in [91]. 120

6.13 Example A: (a)(c)Magnitude errors and (b)(d) phase errors of the filters

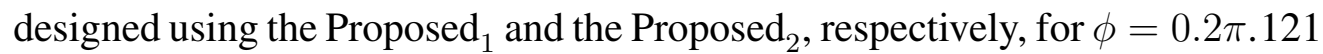

6.14 Example A: the magnitude responses of the VBFD filters with passband edge $\phi$ varying from $0.1 \pi$ to $0.42 \pi$ and an arbitrarily selected fractional delay $d=0.4$ using (a) the Proposed Pand (b) the Proposed $_{2}$, respectively. 122

6.15 Example B: the maximum (a) magnitude errors and (b) phase errors vs. $d$ and $\phi$ of the VBFD filters designed by the Proposed Pr $_{1} \ldots$. . . . . . . . 124

6.16 Example C: the maximum magnitude errors vs. $d$ and $\phi$, of the VBFD filters designed by (a) the Proposed ${ }_{1}$ and (b) the Proposed ${ }_{2}$, respectively. . 126 
6.17 Example C: the maximum phase errors vs. $d$ and $\phi$, of the VBFD filters designed by (a) the Proposed $_{1}$ and (b) the Proposed ${ }_{2}$, respectively. . . . . 127

6.18 Example C: the magnitude responses of the VBFD filters with passband edge $\phi$ varying from $0.06 \pi$ to $0.64 \pi$ and fixed fractional delay $d= \pm 0.3$ using (a) the Proposed ${ }_{1}$ and (b) the Proposed $_{2}$, respectively. . . . . . . . 128

7.1 The implementation of tunable notch filters based on the FFB technique. . 135

7.2 The implementation of simultaneously tunable fractional delay and notch filters based on the FFB technique. . . . . . . . . . . . . 136

7.3 (a) The magnitude response and (b) the phase delay of the FFB based VFD filters. . . . . . . . . . . . . . . . . 137 


\section{List of Tables}

3.1 The numbers of the multiplier block adders required for the synthesis of the coefficient matrices from the proposed technique and the technique in [34] with 9-, 10- and 11-bit precisions, respectively. . . . . . . . 53

4.1 Frequency response error $\delta_{f}$, magnitude error $\delta_{m}$, phase error $\delta_{\Phi}$ and group delay error $\delta_{g}$ at $\omega_{1}$ of $F A$ and $F B$, and the corresponding maximum sample estimation error $\epsilon_{m}$ and the total time domain error energy $e_{t}^{2}$ for single frequency sinusoid input signal. . . . . . . . . . . . 63

4.2 Frequency response error $\delta_{f}$, magnitude error $\delta_{m}$, phase error $\delta_{\Phi}$ and group delay error $\delta_{g}$ at $\omega_{2}$ of $F C$ and $F D$, and the corresponding maximum sample estimation error $\epsilon_{m}$ and the total time domain error energy $e_{t}^{2}$ for single frequency sinusoid input signal. . . . . . . . . . . . . 64

$4.3 e_{f}^{2}$ and $e_{g}^{2}$ given in (4.18), of $F A$ and $F B$ for, and the corresponding time

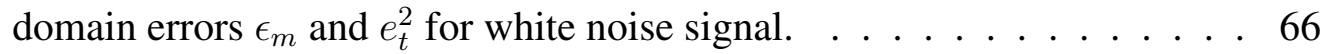

$4.4 e_{f}^{2}$ and $e_{g}^{2}$ given in (4.18), of $F C$ and $F D$ for, and the corresponding time domain errors $\epsilon_{m}$ and $e_{t}^{2}$ for white noise signal. . . . . . . . 67

$4.5 e_{f}^{2}, e_{g}^{2}$ given in (4.18), $\hat{e}_{f}^{2}, \hat{e}_{g}^{2}$ given in (4.20) and (4.21), of $F A$ and $F B$, and the time domain errors $\epsilon_{m}$ and $e_{t}^{2}$ for chirp input signal. . . . . 70

$4.6 e_{f}^{2}, e_{g}^{2}$ given in (4.18), $\hat{e}_{f}^{2}, \hat{e}_{g}^{2}$ given in (4.20) and (4.21), of $F C$ and $F D$, and the time domain errors $\epsilon_{m}$ and $e_{t}^{2}$ for chirp input signal. . . . . . 71 
4.7 The frequency domain error $\hat{e}_{f}^{2}, \hat{e}_{g}^{2}$ given in (4.20) and (4.21), of $F A$ and $F B$, and the time domain errors $\epsilon_{m}$ and $e_{t}^{2}$ for the music input signal. . . 72

4.8 The frequency domain error $\hat{e}_{f}^{2}, \hat{e}_{g}^{2}$ given in (4.20) and (4.21), of $F C$ and $F D$, and the time domain errors $\epsilon_{m}$ and $e_{t}^{2}$ for the music input signal. . . 73

5.1 The complexities of the VFD filters using the proposed technique and the techniques in [65], respectively. . . . . . . . . . . . . . . . 95

5.2 The complexity and the maximum frequency response error of the VFD filter using the proposed technique, the techniques in [70] and [88], respectively. . . . . . . . . . . . . . . . . . 97

6.1 Switch states for delay and shaping filter block $k$ and $\bar{k}=\left\lceil\frac{N(\phi+\gamma)}{2 \pi}\right\rceil$, where $\phi$ is the passband edge, for the shaping structure in Figure 6.6. . . . 108

6.2 Computational complexity and Implementation complexity of Example

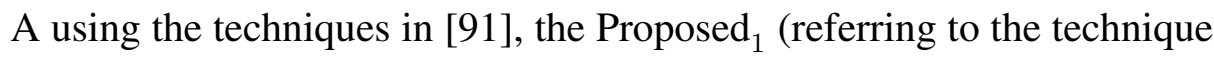

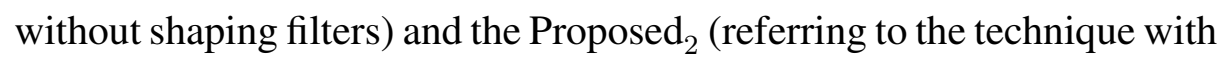
shaping filters). . . . . . . . . . . . . . . . . 130

6.3 Computational complexity and Implementation complexity of Example

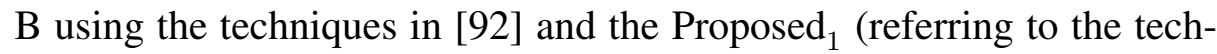
nique without shaping filters). . . . . . . . . . . . 131

6.4 Computational complexity and Implementation complexity of Example

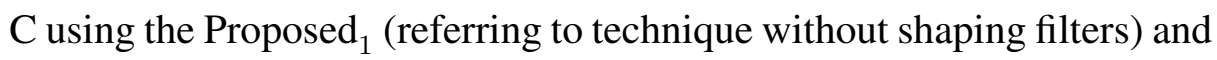
Proposed $_{2}$ (referring to the technique with shaping filters) techniques. . . 132 


\section{List of Abbreviations}

$\begin{array}{ll}\text { DSP } & \text { Digital Signal Processing } \\ \text { FFB } & \text { Fast Filter Bank } \\ \text { FRM } & \text { Frequency Response Masking } \\ \text { IDTFT } & \text { Inverse Discrete-Time Fourier Transform } \\ \text { LP } & \text { Linear Phase } \\ \text { MCM } & \text { Multiple Constant Multiplications } \\ \text { WLS } & \text { Weighted-Least-Squares } \\ \text { VFD } & \text { Variable Fractional Delay } \\ \text { VBFD } & \text { Variable Bandedge and Fractional Delay } \\ \text { XOR } & \text { Exclusive OR }\end{array}$




\section{Chapter 1}

\section{Introduction}

The development of digital filters has been accompanied with the advent and advance of digital signal processing (DSP) [1-6] since last century. Over the ensuing 40 years, digital filters [7-9] have become both a facilitating and an enabling technology, playing DSP roles. We have been blessed to be able to witness and participate in the ongoing phase of the evolution of digital filters.

Digital filters are devices with specified amplitude and phase behaviors to alter the attributes of a signal in digital domain. According to whether the frequency responses of the filters are changeable or not, digital filters can be categorized into two types as fixed digital filters and variable digital filters [10-94]. Generally, compared with variable digital filters, the design of fixed digital filters is relatively straightforward and the complexity of the filters are also low. However, the immutable characteristic of the fixed digital filters limits their practical applications in digital synchronization [95, 96], real-time sampling rate conversions [97-103], speech coding [104-106], modeling and synthesis of musical instruments [107-109], etc [110-116]. In these applications, the desired frequency responses are required to be changed during the signal processing. Hence, variable digital filters have received great attentions from researchers and considerable researches have been done in the design of variable digital filters. Among them, tunable notch 
filters [117-119], variable bandedge filters [10-32], variable fractional delay (VFD) filters [33-88], both variable bandedge and fractional delay (VBFD) filters [89-94], variable fractional order integrator and differentiator [116], variable two-dimensional [22] and three-dimensional [32] digital filters have been studied in-depth. Due to the development of the Farrow structure [48] in 1988, the polynomial approximation of variable characteristics has become very popular in the implementation of variable digital filters to achieve real-time update of coefficient values and accurate control of variable frequency characteristics. The polynomial based Farrow structure has been successfully employed in the syntheses of variable bandedge filters, VFD filters and VBFD filters.

The conventional approaches for the polynomial approximation of VFD filters can be categorized into closed-form approaches and optimization approaches. In the closedform approaches [33-44,73-77], the Lagrange interpolation [33-36, 38-43] is the most attractive technique because it can approximate the required fractional delays accurately if only fractional delays at low frequency are concerned [49]. Many researches have been done to exploit the relations between the filter coefficients to reduce the filter implementation complexity, and the original Farrow structure is modified such that the structure can be used to implement the Lagrange interpolation VFD filter [33-36, 38, 39].

In optimization approaches, the filter coefficients are obtained by minimizing the errors of the frequency responses of the filters deviated from the ideal responses in weighted-least-squares (WLS) sense [60-71, 81-87, 89, 90] or minimax sense [50-59, $79,80,88]$. In the optimization techniques, besides the frequency response errors, the phase errors $[80,81]$, phase delay errors [52, 54, 79], group delay errors $[55,57,71,88]$, or some combinations of the above three errors $[53,59,85]$ have been additionally considered, resulting in different VFD filter performances.

The prevalent polynomial based technique is also applicable to the design of VBFD filters, where two-dimensional (2-D) polynomial approximations of variable bandedges and fractional delays simultaneously are required [89-92]. The resultant VBFD filter is 
further implemented in the nested Farrow structure, i.e., each subfilter in the original Farrow structure is implemented as a Farrow structure. Thus, both bandedge and fractional delay are independently and simultaneously tunable. However, the computational and implementation complexities of the VBFD filters designed using this technique are very high.

The objective of this research is to investigate the design of VFD filters and VBFD filters with low computational and implementation complexities. Throughout this thesis, the computational complexity of a filter refers to the numbers of real multiplications and/or real additions required to generate an output sample, and the implementation complexity of a filter refers to the numbers of real multipliers, adders and delay units required to be implemented when an output sample is generated per clock cycle.

In this thesis, first, the closed-form design of VFD filters using the Lagrange interpolation is investigated. The relation between the subfilter coefficients of the Lagrange interpolation is studied and a more efficient implementation structure is proposed. Second, in the optimization approaches for the design of VFD filters, the different design criteria for the most common application of VFD filters, i.e., the application estimating the instantaneous delayed samples, are investigated. Third, a filter bank approach for the design of VFD filters is proposed. The basic idea of the approach is to split the full band input signals into subbands and each subband is, respectively, shifted by a phase, which is determined by the required fractional delay. The overall fractional delay is achieved by combining all subbands except the band across the normalized frequency $\pi$. This proposed VFD filter can be used effectively to synthesize VBFD filters. A shaping filter technique is introduced into the design of VBFD filters such that the complexity of the VBFD filters is further reduced. 


\subsection{Contributions}

The contributions of this thesis mainly lie in the following:

1. The relations between subfilter coefficients of the Lagrange interpolation are investigated and an efficient implementation structure is proposed to reduce the computational and implementation complexities.

2. An investigation upon the design criteria of VFD filters, including the frequency response error, phase error and group delay error is conducted. For the applications estimating the instantaneous delay samples, the theoretic analysis indicates that the phase error, instead of the group error, is related to the time domain estimation errors. In addition, numerical simulations of real VFD filters with different types of input signals verify the theoretic analysis.

3. A mixed-radix fast filter bank (FFB) is introduced for the design of VFD filters. In this approach, the full band input signal is split into several subbands by the FFB. By shifting each subband a proper phase, VFD is realized by combining necessary subbands. Compared with the existing design techniques, the proposal technique may not achieve the design with less complexity, but it can be incorporated with the variable bandedge characteristic with little extra complexity.

4. Variable bandedge characteristic is incorporated into the FFB approach for the design of VBFD filters. The synthesis of the VBFD filters is achieved by either keeping or discarding the necessary subbands to form the variable bandedges. However, this requires the bandwidth of the individual band of the filter bank is narrower than the transition bandwidth of the VBFD filters. By introducing a shaping filter to the last retained band to form the transition band of the variable digital filters, the bandwidth of the individual band of the filter bank may be relaxed to about twice of the transition bandwidth of the VBFD filters. Compared with the existing VBFD filter design techniques, the proposed approaches significantly reduce the computational complexity of the VBFD filters. 


\subsection{Publications}

The main content of the research contributions can be found in the following papers which have been published or submitted for consideration of publications.

\subsubsection{Journal Papers}

- W. J. Xu and Y. J. Yu, and Håkan Johansson, ”Improved Filter Bank Approach for the Design of Variable Bandedge and Fractional Delay Filters," IEEE Trans. Circuits Syst. I, vol. 61, pp. 764-777, Mar, 2014.

- Y. J. Yu and W. J. Xu, ”Optimization criteria for the design of variable fractional delay filters," IEEE Trans. Circuits Syst. II, vol. 60, pp. 522-526, Aug, 2013.

- Y. J. Yu and W. J. Xu, "Mixed-radix Fast Filter Bank approach for the design of variable digital filters with simultaneously tunable bandedge and fractional delay," IEEE Trans. Signal Processing, vol. 60, pp. 100-111, Jan, 2012.

\subsubsection{Conference Papers}

- W. J. Xu and Y. J. Yu, ”Polynomial implementation structure for Lagrange-type variable fractional-delay filters," in Proc. 2010 IEEE Int. Symp. Circuits and Systems, Paris, France, May 2010, pp. 733-736.

\subsection{Organization}

The remainder of the thesis is organized as follows.

Chapter 2 reviews the basic concepts and the design techniques of variable bandedge filters, VFD filters and VBFD filters.

In Chapter 3, the coefficient relations of the Lagrange-type VFD filters are investigated and an efficient structure is proposed for the implementation. 
The design criteria of VFD filters for the application of instantaneous time domain estimation of delayed samples are investigated in Chapter 4. The frequency response error, phase error and group delay error are considered. The time domain estimation error is theoretically analyzed. It shows that the time domain error is determined by the phase error, instead of the group delay error. In the meanwhile, numerical simulations of VFD filters with some typical input signals verify the theoretic analysis.

In Chapter 5, a novel technique for the design of VBFD filters is proposed, where a mixed-radix FFB is introduced to split the full band input signal into several subbands. By shifting each subband a proper phase and combining necessary subbands, VFD is realized. The 0th stage of the FFB splits the signals into 3 channels by 3 filters, which are compressed and shifted version of the prototype filter of the 0th stage. The optimization of the 0th stage prototype filter of the FFB is proposed, where not only the stopband ripples of the prototype filter is constrained, but the ripples introduced by the other stages in the FFB are also considered.

In Chapter 6, variable bandedge characteristic is introduced into the FFB approach for the design of VFD. The synthesis of the VBFD filters are achieved by either directly combining the necessary bands of the phase shifted FFB or introducing and applying shaping filters to the last retained band to relax the design constraints of the FFB when necessary. In the synthesis of the VBFD filters, the optimization of the prototype filter of the FFB is modified to restrict the magnitude of side lobes.

Chapter 7 concludes the thesis. 


\section{Chapter 2}

\section{Basic concepts and literature reviews}

In this chapter, the basic concepts related to variable digital filters are illustrated. The design approaches of variable digital filters, including variable bandedge filters, VFD filters and VBFD filters are reviewed.

\subsection{Variable Bandedge Filters}

In many applications including telecommunications, digital audio equipment, medical electronics, radar, sonar and control systems, adaptive and tracking systems, spectrum and vibration analyses, format speech synthesizers, it is often desirable to design and implement a digital filter whose bandedge (cutoff frequency) is variable [19].

Taking a lowpass variable bandedge filter with a passband edge $\phi$ varying in a frequency range of $\left[\omega_{l}, \omega_{u}\right]$ as an example, the specifications are generally given as

$$
1-\delta_{m, p} \leq\left|H\left(e^{j \omega}, \phi\right)\right| \leq 1+\delta_{m, p}, \omega \in[0, \phi]
$$

and

$$
\left|H\left(e^{j \omega}, \phi\right)\right| \leq \delta_{m, s}, \omega \in[\phi+\Delta \omega, \pi]
$$

for $\omega_{l} \leq \phi \leq \omega_{u}, 0 \leq \omega_{l}<\omega_{u} \leq \pi-\Delta \omega$ and $\Delta \omega>0$. In (2.1) and (2.2), $\delta_{m, p}$ and $\delta_{m, s}$ denote the passband and stopband magnitude ripple tolerances, respectively, and $\Delta \omega$ is the transition width. In many applications, such as sampling rate conversion, 


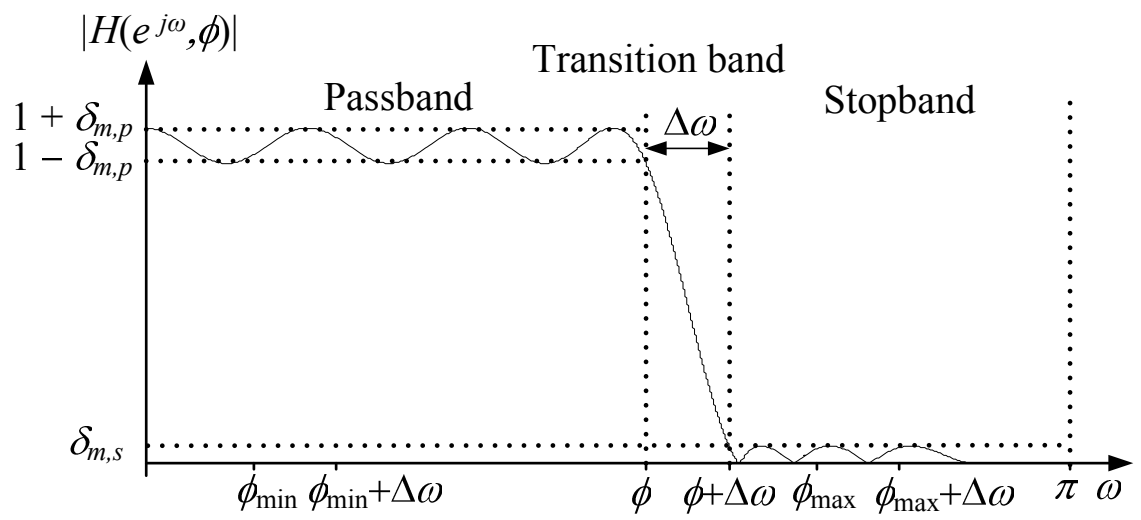

Figure 2.1. The specifications of a lowpass filter with variable bandedges.

image interpolation, etc. $\Delta \omega$ may be fixed. The set of the specifications is illustrated in Figure 2.1 and this is the case studied in this thesis.

\subsection{Design of Variable Bandedge Filters}

The earliest variable bandedge technique, the frequency transformation approach [1015], was first proposed in 1970s and further developed in early 1980s. In this method, each delay element of a prototype filter is replaced by a first-order all pass network. The resulting filter then has an identical frequency response as that of the prototype filter, but on a distorted frequency scale. The frequency transform method suffers from the inherent limitation that the passband width and transition bandwidth cannot be independently controlled. In addition, this method requires special filter structures and the filter length generally increases.

A trigonometric approximation was proposed in $[16,17]$ for the design of variable bandedge filters. In this approach, the filter coefficients were expressed as trigonometric functions of the variable bandedge. Due to the direct control of the filter coefficients, the frequency bands can be independently controlled, but the stopband attenuation is generally small.

Polynomial approximation was proposed in [18] and it received great attentions in the design of variable bandedge filters [19-21,23-30]. In polynomial approximation, 
the filter coefficients are expressed as polynomials of the variable characteristics, i.e., the variable bandedges. The polynomial approximation of the filter coefficients can be efficiently implemented in the Farrow structure [48], which is reviewed in Section 2.5.

The polynomial approximation can accurately control the variable frequency response and conveniently update the coefficients. However, the computational complexity of the Farrow structure is very high.

Recently, frequency response masking (FRM) [120] and fast filter bank (FFB) [121] were used in the design of variable bandedge filters with very sharp transition band and low complexities [31]. In [31], the input signal was decomposed into several bands by using the FFB. The band involved with the transition band of the variable bandedge filter is shifted and shaped by a fixed shaping filter and then shifted back to produce the required transition band. The shaping filter was designed with a very sharp transition band using the FRM technique. Finally the overall variable bandedge filter is achieved by summing up the shifted and shaped bands and the bands in the passband range of the variable bandedge filters. The principles of the FRM and the FFB are reviewed in Section 2.8 .

\subsection{Fractional Delay Filters}

In this section, the concepts of delays including the phase shift, phase delay, group delay and fractional delay are illustrated. Two applications of fractional delay are presented. The design specifications of VFD filters are discussed in the end.

\subsubsection{Phase Shifts and Time Domain Sample Delays}

The frequency components of a signal may be delayed when they pass through a device such as a filter, an amplifier, a loudspeaker, or propagate through space or a medium, such as air. If the time domain sample delay of each frequency component is the same, the device has the property of linear phase; otherwise, different frequency components 


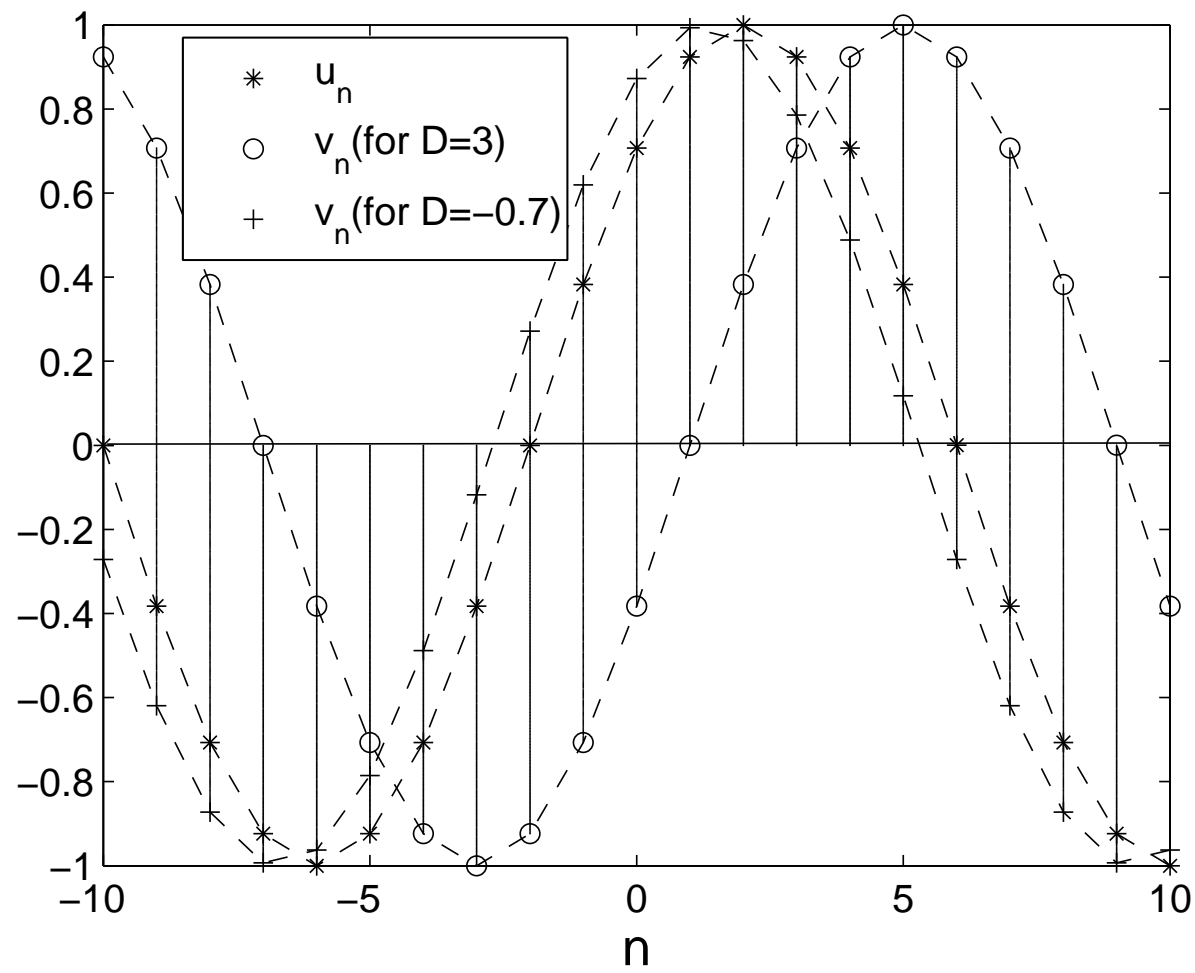

Figure 2.2. The relation between time domain sample delay and phase shifts.

may be delayed by different number of samples. Thus the signals consisting of multiple frequency components may suffer from distortion due to the variation of phase shifts.

The following shows the relation between the phase shift and sample delay of a signal.

Given a signal $u_{n}$ as

$$
u_{n}=A \sin \left(\omega_{0} n+\Phi_{0}\right) \text {, }
$$

its $D$-sample delayed signal $v_{n}$ is obtained as

$$
v_{n}=u_{n-D}=A \sin \left(\omega_{0}(n-D)+\Phi_{0}\right)=A \sin \left(n \omega_{0}-D \omega_{0}+\Phi_{0}\right),
$$

where $D$ is a real number. If $D$ is positive, then the signal $v_{n}$ goes behind $u_{n}$; otherwise advance of $u_{n}$. According to (2.3) and (2.4), it can be found that $v_{n}$ physically has an amount of phase shift $-D \omega_{0}$ compared with $u_{n}$, resulting in the sample delay of $D$. Figure 2.2 illustrates the signals $u_{n}$ and $v_{n}$ for $A=1, \omega_{0}=\frac{\pi}{8}, \Phi_{0}=0.25 \pi, D=3$ and $D=-0.7$. 


\subsubsection{Phase Delay and Group Delay}

The phase response $\Phi(\omega)$ of a digital filter $H\left(e^{j \omega}\right)$, is given as

$$
\Phi(\omega)=\angle H\left(e^{j \omega}\right)
$$

It applies a shift to the phase of each sinusoid component of the input signal.

Phase delay and group delay, denoted as $\tau_{p}$ and $\tau_{g}$, are defined as

$$
\tau_{p}=-\frac{\Phi(\omega)}{\omega}
$$

and

$$
\tau_{g}=-\frac{\partial \Phi(\omega)}{\partial \omega}
$$

respectively.

From (2.6), it is obvious that the phase delay is the normalized phase shift by the frequency. On the other hand, the physical meaning of the group delay of (2.7) is not so straightforward and can be illustrated by a signal $x_{n}$ containing two frequency components $\omega_{1}$ and $\omega_{2}$, i.e., $\omega_{1}=0.025 \pi$ and $\omega_{2}=0.03 \pi$, given as

$$
x_{n}=\cos \left(\omega_{1} n\right)+\cos \left(\omega_{2} n\right) .
$$

The waveform of $x_{n}$ is shown in Figure 2.3 in the solid line. By using a trigonometric identity, $x_{n}$ can be written as

$$
\begin{aligned}
x_{n} & =2 \cos \left(\frac{\omega_{1}-\omega_{2}}{2} n\right) \cos \left(\frac{\omega_{1}+\omega_{2}}{2} n\right) \\
& =x_{e} \cos \left(\frac{\omega_{1}+\omega_{2}}{2} n\right)
\end{aligned}
$$

where $x_{e}=2 \cos \left(\frac{\omega_{1}-\omega_{2}}{2} n\right)$ is the envelop of $x_{n}$ as shown in Figure 2.3(a) in the dash-dot line. If the signal of the two frequency components $\omega_{1}$ and $\omega_{2}$ are delayed by different amounts of phases, e.g., $\Phi_{1}=0.15 \pi$ and $\Phi_{2}=0.5 \pi$, respectively, without any magnitude attenuation, the output $y_{n}$ is given by

$$
y_{n}=\cos \left(\omega_{1} n-\Phi_{1}\right)+\cos \left(\omega_{2} n-\Phi_{2}\right) \text {, }
$$




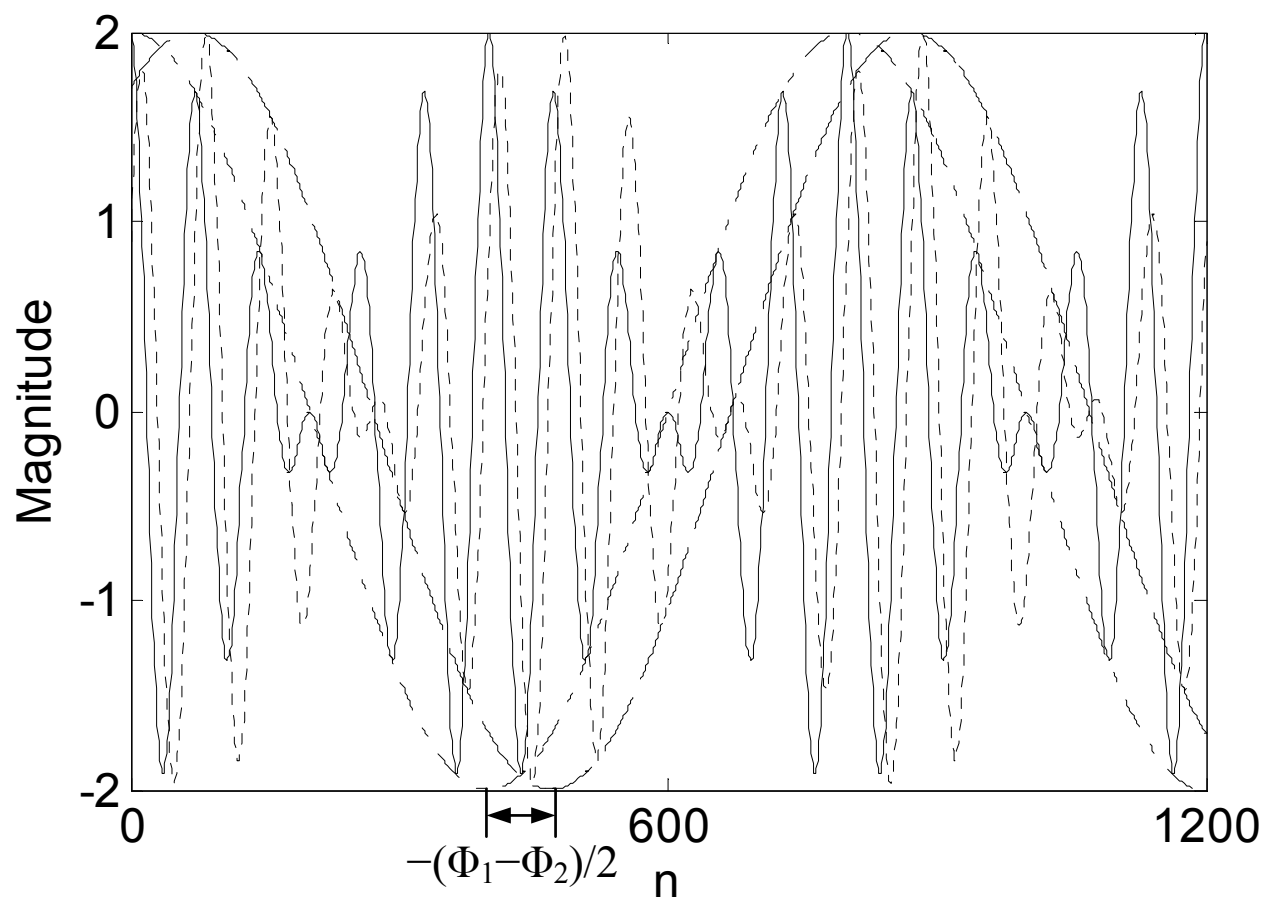

Figure 2.3. Plots of signal $x_{n}$ (solid line), its envelop $x_{e}$ (dash-dot line), $y_{n}$ (dot line) and the envelop $y_{e}$ (dash line).

as shown in Figure 2.3 in the dot line. Using the same trigonometric identity to (2.10), (2.10) is rewritten as

$$
\begin{aligned}
y_{n} & =2 \cos \left(\frac{\omega_{1}-\omega_{2}}{2} n-\frac{\Phi_{1}-\Phi_{2}}{2}\right) \cos \left(\frac{\omega_{1}+\omega_{2}}{2} n-\frac{\Phi_{1}+\Phi_{2}}{2}\right) \\
& =y_{e} \cos \left(\frac{\omega_{1}+\omega_{2}}{2} n-\frac{\Phi_{1}+\Phi_{2}}{2}\right)
\end{aligned}
$$

where $y_{e}=2 \cos \left(\frac{\omega_{1}-\omega_{2}}{2} n-\frac{\Phi_{1}-\Phi_{2}}{2}\right)$ is the envelop of $y_{n}$, as plotted in Figure 2.3 in the dash line. From Figure 2.3, it can be found that the phase shift between $y_{e}$ and $x_{e}$ is $-\frac{\Phi_{1}-\Phi_{2}}{2}$. In the meanwhile, from (2.8) and (2.11), it can be found that the group delay $\tau_{g}$

$$
\tau_{g}=-\frac{\Phi_{1}-\Phi_{2}}{\omega_{1}-\omega_{2}}=-\frac{\Delta \Phi}{\Delta \omega}
$$

measures the delay of the envelop as shown in Figure 2.3. Meanwhile, the signal sample delay cannot be identified from the figure because the two frequency components are delayed by different sample delays, i.e., the signal is distorted due to the nonlinear phase. 


\subsubsection{Fractional Delay}

It is conceptually simple to delay a continuous-time signal $x_{c}(t)$ by an amount $t_{D}$. Denoting $L_{c}$ as a linear operator for a continuous-time ideal delay, the output $y_{c}(t)$ is yielded as

$$
y_{c}(t)=L_{c}\left\{x_{c}(t)\right\}=x_{c}\left(t-t_{D}\right)
$$

When an uniformly sampled bandlimited digital signal is delayed by a noninteger sample $D$ in time domain, the situation is more complicated. For a non-integer value $D$, consisting of an integer part and a fraction part as

$$
D=\operatorname{Int}(D)+\operatorname{Fra}(D)
$$

if (2.13) is converted into discrete time by sampling $x_{c}(t)$ at time instant $t=n T$, where $n$ is an integer and $T$ is the sampling interval, (2.13) in the form of discrete time can be written as

$$
y(n)=x(n-D) .
$$

When $D$ is an integer value, the output sample can be produced by passing the input signal through a delay chain. However, when $D$ is a noninteger value, $x(n-D)$ is not defined and its value lies somewhere between two discrete samples. In this case, interpolation has to be applied to obtain $x(n-D)$. Figure 2.4 illustrates the input and output samples for $D=1.3$.

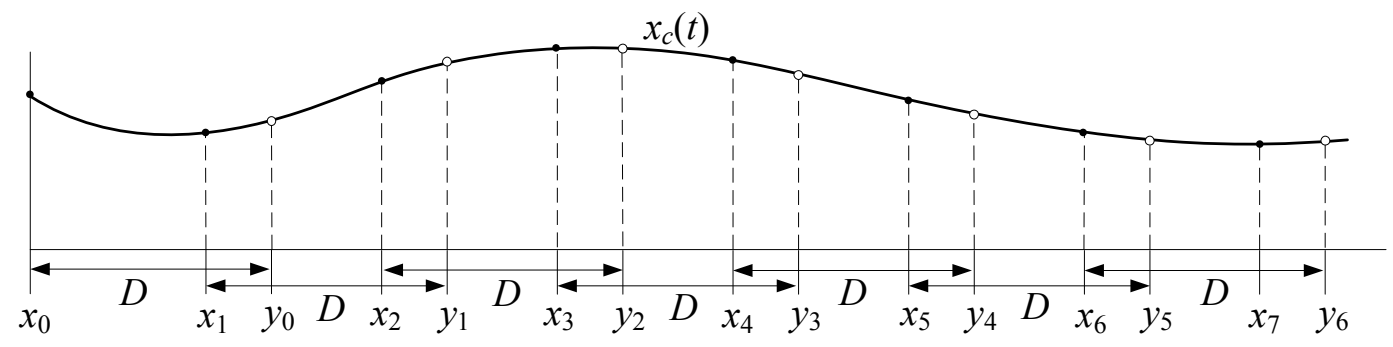

Figure 2.4. The concept of fractional delay. 
In digital signal processing, a fractional delay can be realized by a fractional delay filter. In the following sections, fractional delay filters and variable fractional delay filters are introduced.

\subsubsection{Fractional Delay Filters}

To realize the time domain delay described in (2.15), an ideal fractional delay filter has a $z$-transform transfer function given by

$$
\hat{H}(z)=\frac{Y(z)}{X(z)}=z^{-D}
$$

where $Y(z)$ and $X(z)$ are the $z$-transform of the output and input signal of a fractional delay filter.

Setting $z=e^{j \omega}$, the frequency response of the ideal fractional delay filter is given by

$$
\hat{H}\left(e^{j \omega}\right)=e^{-j \omega D},|\omega|<\pi
$$

The frequency response is a complex-valued function which specifies the magnitude and phase responses as

$$
\left|\hat{H}\left(e^{j \omega}\right)\right|=1
$$

and

$$
\hat{\Phi}(\omega)=-D \omega
$$

respectively, where $\hat{\Phi}(\omega)$ is the phase response of the ideal fractional delay filter, given by

$$
\hat{\Phi}(\omega)=\angle \hat{H}\left(e^{j \omega}\right)
$$

It can be seen that the ideal fractional delay filter is a linear-phase allpass system. Its phase delay and group delay, denoted as $\hat{\tau}_{p}(\omega)$ and $\hat{\tau}_{g}(\omega)$, are given by

$$
\begin{gathered}
\hat{\tau}_{p}(\omega)=-\frac{\hat{\Phi}(\omega)}{\omega}=D \\
\hat{\tau}_{g}(\omega)=-\frac{\partial \hat{\Phi}(\omega)}{\partial \omega}=D .
\end{gathered}
$$


respectively. From these results, it can be seen that ideally, a fractional delay filter passes all the frequency components of the incoming signal with the same sample delay $D$.

The impulse response of the ideal fractional delay filter can be derived from (2.17) by the inverse discrete-time Fourier transform (IDTFT), given as

$$
\hat{h}(n)=\frac{1}{2 \pi} \int_{-\pi}^{\pi} \hat{H}\left(e^{j \omega}\right) e^{j \omega n} d \omega=\frac{1}{2 \pi} \int_{-\pi}^{\pi} e^{j \omega(n-D)} d \omega=\operatorname{sinc}(n-D) .
$$

According to (2.23), due to the zero-crossing property of the sinc function at integer values except 0 , the impulse response $\hat{h}(n)$ is zero at all $n \neq D$ and $\hat{h}(D)=1$ for an integer delay $D$, i.e.,

$$
\hat{h}(n)= \begin{cases}1, & \text { for } n=D \\ 0, & \text { otherwise }\end{cases}
$$

When $D$ is a noninteger value, the impulse response $\hat{h}(n)$ has non-zero value for any $n$, i.e., $\hat{h}(n) \neq 0$ for any $n$.

As a result, the impulse response $\hat{h}(n)$ for a noninteger delay $D$ is a shifted and sampled version of the sinc function with infinite length. In other words, the ideal fractional delay cannot be implemented causally due to the infinite length of the impulse response. Therefore, finite impulse response (FIR) approximation for the sinc function has to be employed to obtain a realizable fractional delay filter.

The ideal impulse responses of the integer delay filter for $D=1$ and the truncated ideal impulse response of the fractional delay filter for $D=1.3$ are shown in Figures 2.5(a) and (b), respectively, for $n=-3,-2, \ldots, 5$. Practically, fractional delay filters are usually realized using traditional time-domain or frequency-domain interpolation methods $[37,49,122]$. 


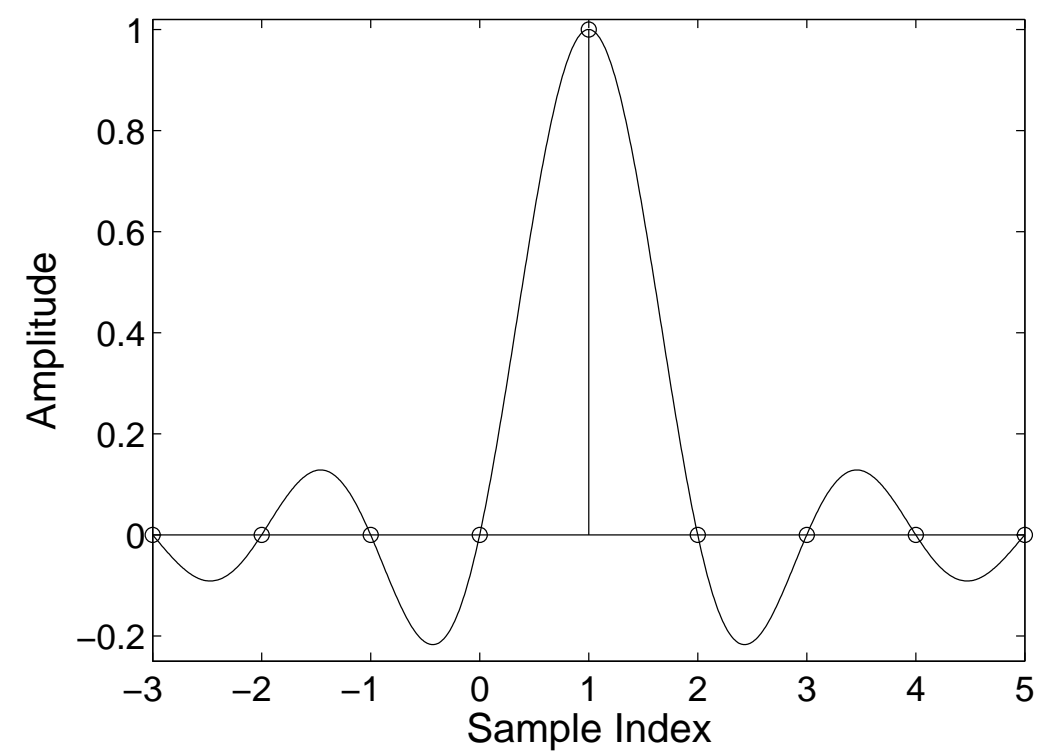

(a)

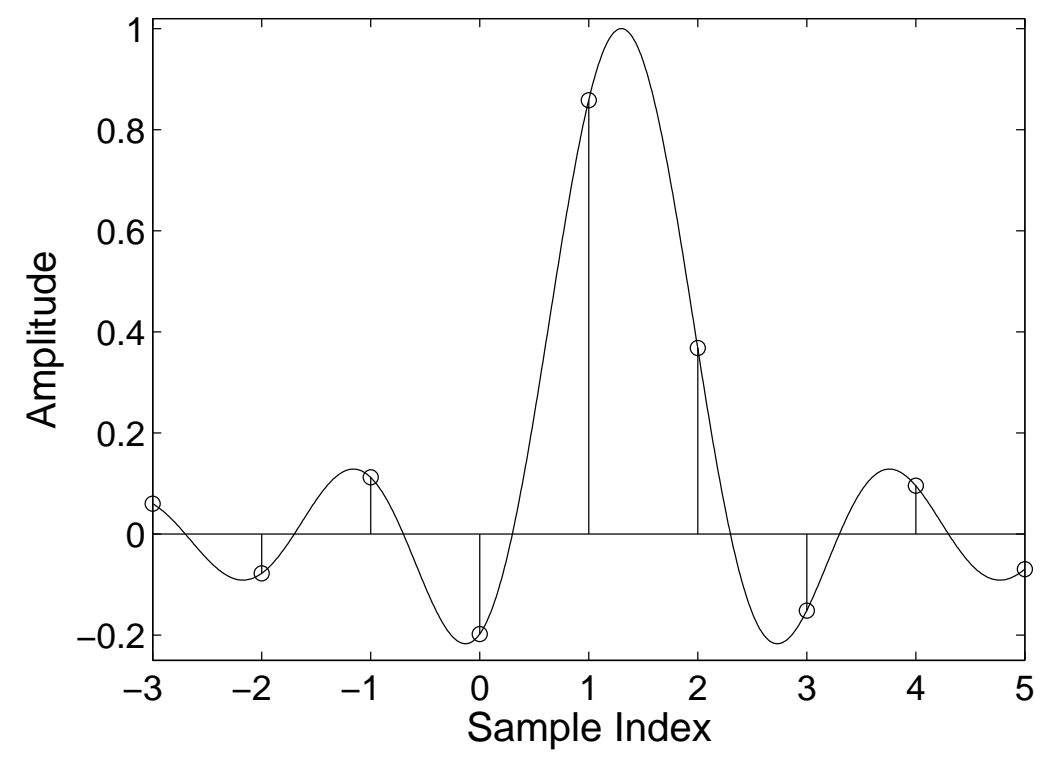

(b)

Figure 2.5. Impulse responses $\hat{h}(n)$ of an ideal delay filter with a delay (a) $D=1$ and (b) $D=1.3$, respectively. 


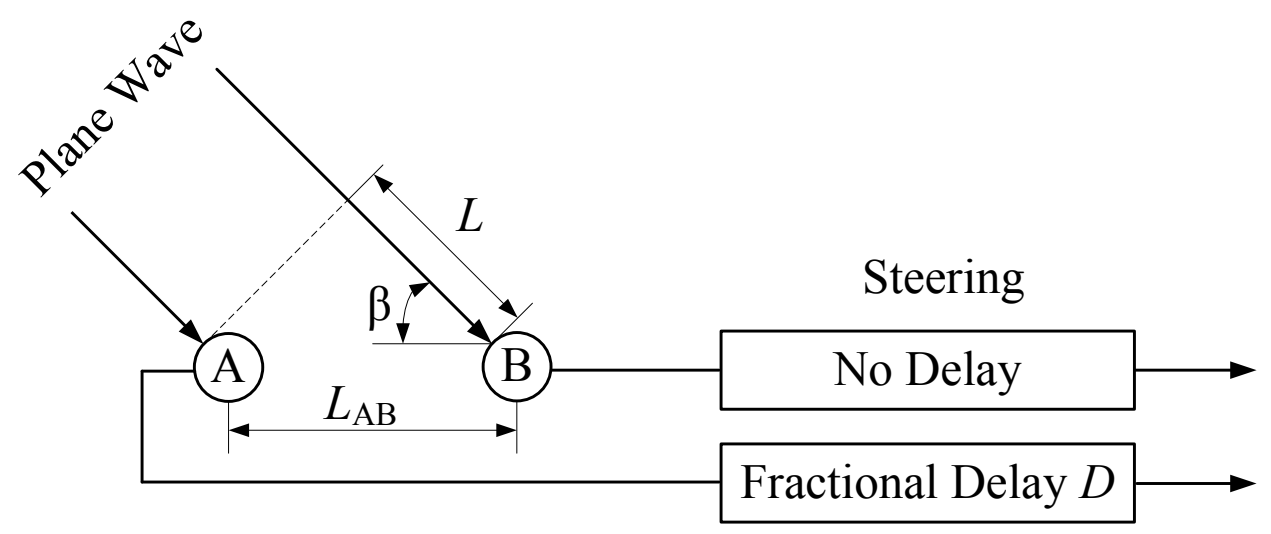

Figure 2.6. Fractional delay in beamforming

\subsubsection{Applications of Fractional Delays}

Fractional delay filters basically are used to estimate the time domain samples that do not occur at the sampling moment. They have many applications in DSP, including samplingrate conversion, timing adjustment of digital modems, music synthesis, array beamforming, etc. Among many others, two of the important applications of fractional delay filters are illustrated in the following.

\subsubsection{Array Beamforming}

Conventionally, beamforming in antenna or transducer arrays is realized by delaying and summing input signals received by the array elements. In such beamforming systems, the requirement that the signals are to be delayed by a fraction of a sample period is a critical part of array beamforming. Figure 2.6 shows two microphones A and B with a plane wave approaching. The wavefront reaches microphone A earlier than microphone B. To virtually steer the array beam to point to the plane wave's direction of arrival, the signal received at microphone A must be delayed properly.

Given the microphone distance of $L_{A B}=0.25 \mathrm{~m}$ and the plane wave arrival direction of $\beta=\frac{\pi}{4}$, the wavefront travels a further distance $L=L_{A B} \cos \beta=0.1768 \mathrm{~m}$ to reach microphone $\mathrm{B}$ after it reaches microphone A. Therefore, to align the two microphone 


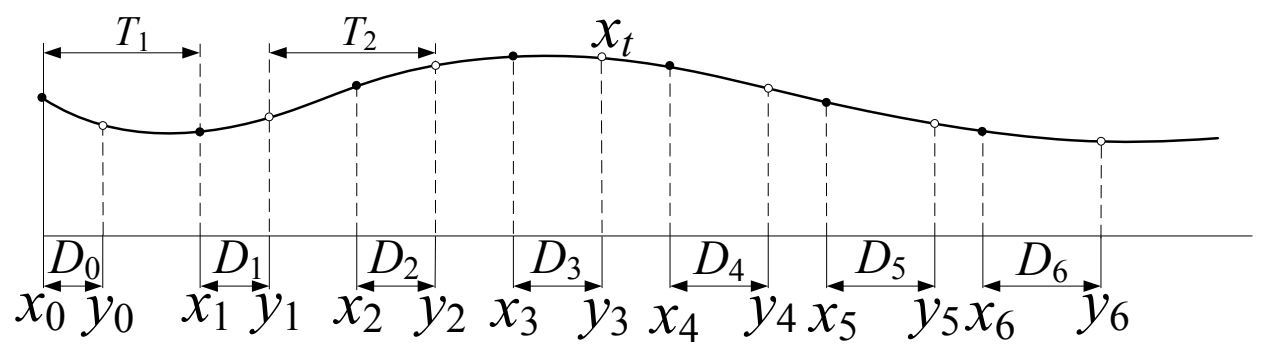

Figure 2.7. Fractional delay in sampling rate conversion

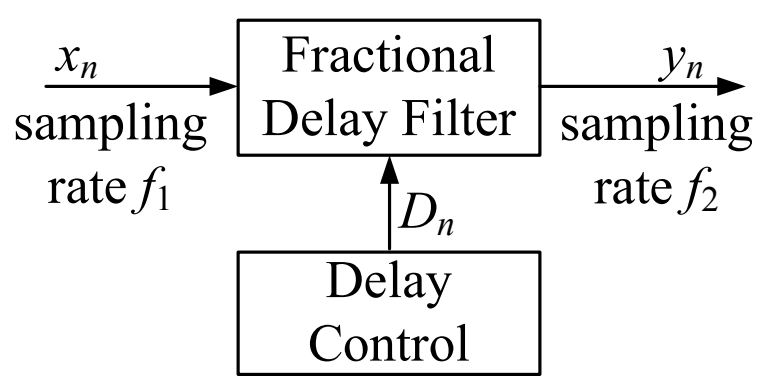

Figure 2.8. Block diagram of an incommensurate-ratio sampling-rate converter

signals, the signal from microphone A must be delayed by $0.5149 \mathrm{~ms}$ given that the speed of sound is $343.3 \mathrm{~m} / \mathrm{s}$. If the microphone signals are sampled at a rate of 48000 samples per second, the delay of $0.5149 \mathrm{~ms}$ is equivalent to a delay of $D=24.717$ samples and hence a fractional sample delay is required.

\subsubsection{Incommensurate Sampling Rate Conversion}

Sampling rate conversion is one of the standard problems in digital signal processing. A well-known example is the conversion between the different sampling frequencies used in digital audio. Typical sampling rates for digital audios are $44.1 \mathrm{kHz}$ (used in the CDquality audio), $48 \mathrm{kHz}$ (used by the DAT recorders), $32 \mathrm{kHz}, 22.05 \mathrm{kHz}$ and $11.025 \mathrm{kHz}$ among others (see, e.g., [123]).

Sampling rate conversion of a digital signal can be realized by down and/or up sampling by integer factors, if the sampling conversion factor is an integer or a ratio of small 
integers $[1,3]$. However, such approach is not feasible if the conversion factor is incommensurate. A possible solution is to use fractional delay filters [102, 124-127].

As shown in Figure 2.7, let $x_{n}$ for $n=0,1, \ldots, 6$ be the digital sequence of the continuous-time signal $x_{t}$ sampled at time interval $T_{1}$. If the digital sequence $y_{n}$ for another sampling interval $T_{2}$ is desired, $y_{n}$ can be obtained by delaying $x_{n}$ by different fractional delay $D_{n}$ as shown in Figure 2.7.

The arbitrary sample rate conversion thus can be implemented by the structure given in Figure 2.8. The delay control unit computes the required fractional delay for each sample, and the fractional delay filter approximates the delayed samples in real time.

From this application, it can be seen that in many cases, not only fractional delays are required, the fractional delays may also be variable in the course of processing.

\subsection{Variable Fractional Delay Filters}

The example in Section 2.3.5.2 shows that the delay of the fractional delay filter is required to vary during the course of signal processing. Some other applications may be found in [104-116]. Hence, the design of variable fractional delay (VFD) filters is necessary.

In the design of VFD filters, given the frequency response of the ideal VFD filters $\hat{H}\left(e^{j \omega}, D\right)=e^{-j \omega D}$ in the frequency range of interest, e.g., $\left[0, \omega_{u}\right]$, the specifications of an $N$ th VFD filters $H\left(e^{j \omega}, D\right)$ are generally given as

$$
-\delta_{m} \leq\left|H\left(e^{j \omega}, D\right)\right|-\left|\hat{H}\left(e^{j \omega}, D\right)\right| \leq \delta_{m}
$$

and

$$
|\Phi(\omega, D)-(-\omega D)| \leq \delta_{\Phi}
$$

where $\omega \in\left[0, \omega_{u}\right]$ and $D \in[0, N]$. In (2.25) and (2.26), $\delta_{m}$ and $\delta_{\Phi}$ denote the allowable maximum passband magnitude and phase errors, respectively. The set of the specifications is illustrated in Figure 2.9. 


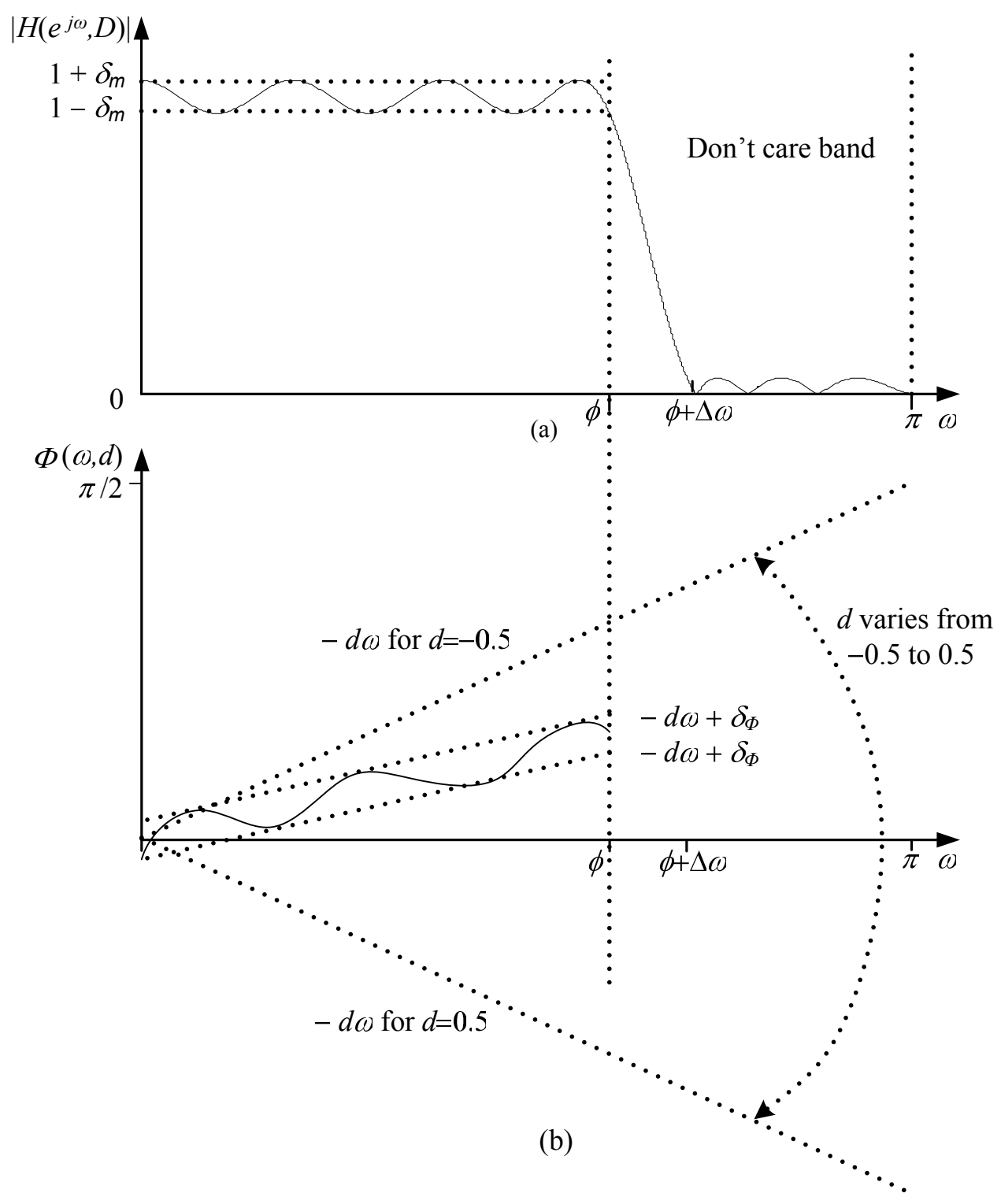

Figure 2.9. The specifications of a VFD filter. 
In some literature, the group delay and/or phase delay errors are also constrained as

$$
\left|-\frac{\partial \Phi(\omega, D)}{\partial \omega}-D\right| \leq \delta_{g}
$$

and

$$
\left|-\frac{\Phi(\omega, D)}{\omega}-D\right| \leq \delta_{p}
$$

where $\delta_{g}$ and $\delta_{p}$ denote the group delay and phase delay error tolerance, respectively. In Chapter 4, an investigation upon the impacts of these three measurements on the time domain sample estimation is presented.

\subsection{Design of Variable Fractional Delay Filters}

The accurate approximation of the ideal variable frequency response of the VFD filter became practical after Farrow proposed the technique to represent each FIR filter coefficient as a polynomial of the fractional delay [48], i.e.,

$$
h(n, D)=\sum_{m=0}^{M} c_{m}(n) D^{m}, n=0, \ldots, N \text { and } 0 \leq D \leq N .
$$

In (2.29), $h(n, D)$ is the $n$th coefficient of the VFD filter, $M$ and $N$ are the polynomial order and the filter order, respectively, and $c_{m}(n)$ is the $m$ th polynomial coefficient. Thus, the $z$-transform transfer function of the VFD filter is expressed as

$$
\begin{aligned}
H(z, D) & =\sum_{n=0}^{N} h(n, D) z^{-n} \\
& =\sum_{n=0}^{N} \sum_{m=0}^{M} c_{m}(n) D^{m} z^{-n} \\
& =\sum_{m=0}^{M} \sum_{n=0}^{N} c_{m}(n) z^{-n} D^{m} \\
& =\sum_{m=0}^{M} C_{m}(z) D^{m} .
\end{aligned}
$$

where

$$
C_{m}(z)=\sum_{n=0}^{N} c_{m}(n) z^{-n}
$$




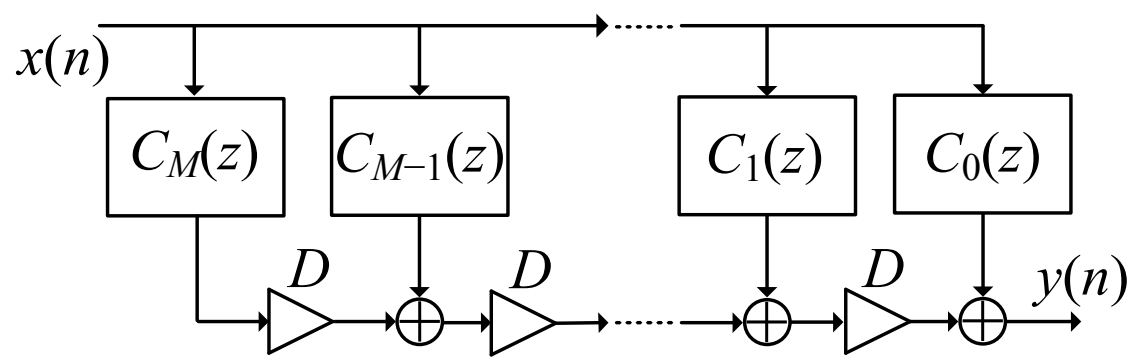

Figure 2.10. The Farrow structure.

The polynomial approximation of the VFD can be efficiently implemented by the structure proposed by Farrow [48]. The structure later was named after Farrow as the Farrow structure as shown in Figure 2.10.

In Figure 2.10, $C_{m}(z)$ for $m=0, \ldots, M$ is the $m$ th subfilter of the Farrow structure. The polynomial approximation error $E\left(e^{j \omega}, D\right)$ is given by

$$
E\left(e^{j \omega}, D\right)=H\left(e^{j \omega}, D\right)-\hat{H}\left(e^{j \omega}, D\right)
$$

where $H\left(e^{j \omega}, D\right)$ is the frequency response of (2.30), given by

$$
H\left(e^{j \omega}, D\right)=\sum_{n=0}^{N} h(n, D) e^{-j \omega n} .
$$

A two-rate approach [88] based on the Farrow structure was recently proposed to more effectively realize the VFD filters. In this approach, the input signal is first upsampled by a factor of two, then filtered through a half band filter $Q(z)$ cascaded by a fractional delay filter with delay $2 D$, and finally downsampled to the original signal sampling rate. With such arrangements, the bandwidth requirement of the VFD filter is relaxed and hence the filter complexity is reduced.

Besides the polynomial approximation based on the Farrow structure, the VFD filters may also be designed using differentiator [72]. This technique requires fewer distinct filter coefficients and less optimization complexities, but the computational complexity required for each output sample is not reduced.

Since the development of the polynomial approximation and Farrow structure, many polynomial approximation techniques for the design of VFD filters have been proposed, 
including both the closed-form approaches and the optimization approaches.

\subsubsection{Closed-form Approaches}

The most popular polynomial based technique for the closed-form designs of VFD filters is the maximally flat FIR filters [33-44]. In the maximally flat technique, the coefficients of the $N$-th order VFD filter are obtained by setting the error function in (2.32) and its $k$-th derivatives for $k=1, \ldots, N$ to zero at the frequency $\omega=\omega_{0}$ of interest, i.e.,

$$
\left.\frac{\partial^{k} E\left(e^{j \omega}, D\right)}{\partial \omega^{k}}\right|_{\omega=\omega_{0}}=0 \text { for } k=0,1,2, \cdots, N
$$

Substituting (2.17) and (2.33) into (2.34), (2.34) can be simplified as

$$
\left.\frac{\partial^{k}}{\partial \omega^{k}}\left[\sum_{n=0}^{N} h(n, D) e^{-j \omega n}-e^{-j \omega D}\right]\right|_{\omega=\omega_{0}}=0 \text {, for } k=0,1,2, \cdots, N .
$$

The $N+1$ simultaneous equations in (2.35) can be simplified to

$$
\sum_{n=0}^{N} e^{-j \omega_{0} n} n^{k} h(n, D)=D^{k} e^{-j \omega_{0} D} \text { for } k=0,1,2, \cdots, N
$$

resulting in a closed-form solution, given by

$$
h(n, D)=e^{j \omega_{0}(n-D)} \prod_{k=0 \text { and } k \neq n}^{N} \frac{D-k}{n-k}, \text { for } n=0,1,2, \cdots, N .
$$

A special case of the maximally flat technique for the design of VFD filters is the Lagrange interpolation [33-36,38-43], which was independently developed by Hermanowicz [40]. In the Lagrange interpolation, given a set of $N+1$ known samples $f\left(x_{n}\right)$ of the variable $x_{n}$ at values $n=0, \ldots, N$, the interpolated sample at $x$ is given by an $N$-th order polynomial $y(x)$

$$
y(x)=\sum_{n=0}^{N} l_{n}(x) f\left(x_{n}\right)
$$

where

$$
l_{n}(x)=\frac{\left(x-x_{0}\right) \cdots\left(x-x_{n-1}\right)\left(x-x_{n+1}\right) \cdots\left(x-x_{N}\right)}{\left(x_{n}-x_{0}\right) \cdots\left(x_{n}-x_{n-1}\right)\left(x_{n}-x_{n+1}\right) \cdots\left(x_{n}-x_{N}\right)} .
$$


In (2.39), the numerator is an $N$-th order polynomial having zeros at $x=x_{m}$ for all $m$ from 0 to $N$, except $m=n$. The denominator is a constant normalizing $l_{n}\left(x_{n}\right)$ to 1 . Thus, (2.39) can be simplified as

$$
l_{n}\left(x_{m}\right)=\delta_{n m}= \begin{cases}1, & n=m \\ 0, & n \neq m\end{cases}
$$

Therefore, given the $N+1$ samples of the frequency response of the ideal VFD filter for noninteger delay $D(0 \leq D \leq N)$, the function $\hat{H}\left(e^{j \omega}, D\right)$ can be approximated by

$$
H\left(e^{j \omega}, D\right)=\sum_{n=0}^{N} h(n, D) e^{-j \omega n}
$$

using the Lagrange interpolation, where $h(n, D)$ for $n=0, \ldots, N$ are the Lagrange fractional delay impulse response, given by

$$
h(n, D)=\prod_{k=0 \text { and } k \neq n}^{N} \frac{D-k}{n-k}, \text { for } n=0,1, \cdots, N .
$$

Comparing (2.37) and (2.42), it shows that the Lagrange interpolator is a maximally flat filter with the maximally flat frequency at $\omega_{0}=0$. A further review of the Lagrange interpolation VFD filter is given in Chapter 3.

The maximally flat design can approximate the fractional delayed sample with low errors for signals at frequency $\omega=\omega_{0}$. However, the frequency response is deteriorated when the frequencies are away from $\omega_{0}$. Moreover, it should be noted that except the desired frequencies of $\omega_{0}=0$ and $\omega_{0}=\pi$, the coefficient values $h(n, D)$ are complex. Complex FIR filters are computationally more expensive than real-coefficient filters of the same order.

\subsubsection{Optimization Approaches}

While the closed-form approaches can design VFD filter quickly, they cannot control the response precisely for given frequency ranges; numerical optimization approaches 
are generally used when accurate control of the response is necessary. In the optimization techniques, the frequency response errors in (2.32) are minimized in the weightedleasted-square sense or minimax sense.

The weighted-leasted-square method was proposed for the design of VFD filters by Tarczynski et al. [61]. In this method, the filter coefficients were obtained by minimizing the objective function $J$, given as

$$
J=\sum_{\omega_{m} \in \boldsymbol{\Omega}} \sum_{p_{n} \in \mathbf{P}} w\left(\omega_{m}, p_{n}\right)\left|E\left(e^{j \omega_{m}}, p_{n}\right)\right|^{2}
$$

where $\Omega$ and $\mathbf{P}$ are the sets containing dense grids of the normalized frequency and the fractional delays over the desired range, respectively. In $(2.43), w\left(\omega_{m}, p_{n}\right)$ is a nonnegative separable two-dimensional weighting function, which is the product of two weighting functions $w_{1}\left(\omega_{m}\right)$ and $w_{2}\left(p_{n}\right)$, i.e.,

$$
w\left(\omega_{m}, p_{n}\right)=w_{1}\left(\omega_{m}\right) w_{2}\left(p_{n}\right)
$$

In $[62,64]$, a technique without discretization of frequency and fractional delay grid points is proposed by minimizing

$$
J=\int_{0}^{\omega_{u}} \int_{0}^{0.5} w(\omega, p)\left|E\left(e^{j \omega}, p\right)\right|^{2} d p d \omega .
$$

This was achieved by using matrix trace properties to evaluate $J$. Compared with the method in [61], the WLS method proposed in $[62,64]$ reduces the computational complexity required in the design process and achieves better design accuracy. Later, the symmetric property of the coefficients of the subfilters was exploited in $[34,65,66]$, further reducing the complexity both in the design process and filter implementations. In [69], the peak-constrained WLS method was proposed, where the objective function $J$ in (2.45) was minimized and in the meanwhile, the maximum derivation of the frequency response was constrained.

Besides the WLS approach, minimax strategies are also developed for the deign of 
VFD filters. In [51,52], the subfilter coefficients were obtained by minimizing the maximum phase delay errors with the constraint of the maximum magnitude errors, while in [55-58], the maximum frequency response errors were minimized and the group delay errors were constrained additionally in $[55,57]$. In the meanwhile, the modified Farrow structure was introduced by properly changing the delay parameter $D$ to $d=D-\frac{N}{2}$ so that each subfilter was linear phase and hence the complexity was reduced due to the coefficient symmetry. In [53], the linear-phase subfilters were designed separately in minimax sense and the overall filter was further optimized using nonlinearly optimization algorithms. In this technique, the subfilters were designed with different orders and the overall arithmetic complexity was reduced. Before that, the subfilters in the Farrow structure were all of the same length. In [54], two subfilters of the Farrow structure were first designed and then the remaining ones were generated by properly combining these two subfilters with some additional short filters, pure delay units, adders, and multipliers. This technique further reduced the computational and implementation complexities.

Besides the above WLS and minimax designs, other techniques $[44,47]$ have been proposed as well. In [44], a differentiator bank was proposed for the design of VFD filters. In this approach, the problem was transformed into the design of differentiators with different orders using Taylor series expansion. In [47], Pei et al. proposed a cepstrum-based approach for the design of VFD filters in the maximally-flat sense, where the criteria of the VFD filter on the frequency responses were transformed to a set of linear equations to solve the truncated complex cepstrum.

\subsection{Variable Bandedge and Fractional Delay Filters}

Some applications, e.g., sampling rate conversion between different frequency pairs, require both the bandedge and the fractional delay of the digital filter to be tunable. A straightforward way to realize the variable bandedge and fractional delay (VBFD) filters 
is to cascade a variable bandedge filter with an VFD filter. However, this approach results in transient problem. Hence a filter system with simultaneously variable bandedge and VFD characteristics is highly desirable.

In this thesis, a lowpass VBFD filter is considered. The specifications of the variable filter including magnitude and phase requirements are given as follows. For magnitude response, the variable filter is a lowpass filter with a variable passband edge $\phi$ and a fixed transition width $\Delta \omega$. The maximum passband and stopband magnitude ripples are $\delta_{m, p}$ and $\delta_{m, s}$, respectively. $\phi$ is variable in a range of $\left[\omega_{l}, \omega_{u}\right]$, whereas $\Delta \omega, \delta_{m, p}, \delta_{m, s}, \omega_{l}$ and $\omega_{u}$ are fixed for a given design. Meanwhile, the ideal phase response of the variable filter is $-d \omega$ (i.e., a variable fractional delay) for a fraction $d$ varying in a range of $[-0.5,0.5]$; the maximum deviation of the phase from the ideal phase is $\delta_{\Phi}$ in the passband. In some designs $[55,57,71]$, the maximum deviation of the group delay from the ideal delay $\delta_{g}$ in the passband is also concerned. Thus, for the frequency response of the variable filter given as

$$
H\left(e^{j \omega}, \phi, d\right)=\left|H\left(e^{j \omega}, \phi, d\right)\right| e^{j \Phi(\omega, \phi, d)},
$$

it satisfies the following constraints:

$$
\begin{aligned}
|| H\left(e^{j \omega}, \phi, d\right)|-1| & \leq \delta_{m, p}, \text { for } \omega \in[0, \phi] \\
\left|H\left(e^{j \omega}, \phi, d\right)\right| & \leq \delta_{m, s}, \text { for } \omega \in[\phi+\Delta \omega, \pi] \\
|\Phi(\omega, \phi, d)-(-d \omega)| & \leq \delta_{\Phi}, \quad \text { for } \omega \in[0, \phi] \\
\left|\frac{\partial \Phi(\omega, \phi, d)}{\partial \omega}-(-d)\right| & \leq \delta_{g}, \quad \text { for } \omega \in[0, \phi]
\end{aligned}
$$

for $\omega_{l} \leq \phi \leq \omega_{u},-0.5 \leq d \leq 0.5,0 \leq \omega_{l}<\omega_{u} \leq \pi-\Delta \omega$, and $\Delta \omega>0$. The magnitude specifications and phase specifications are illustrated in Figures 2.11. 


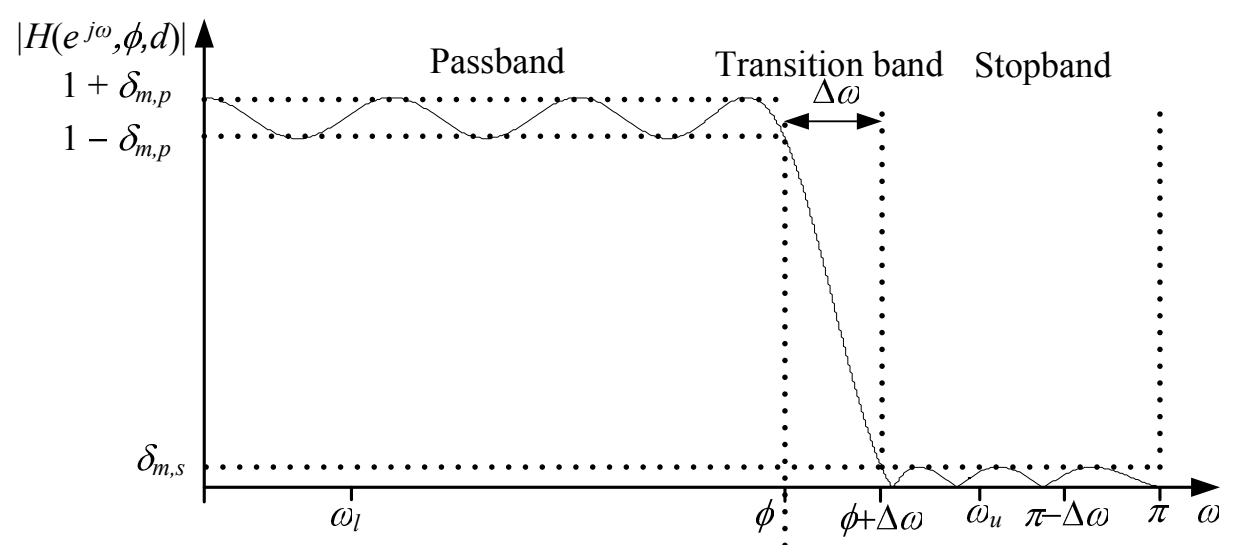

(a)

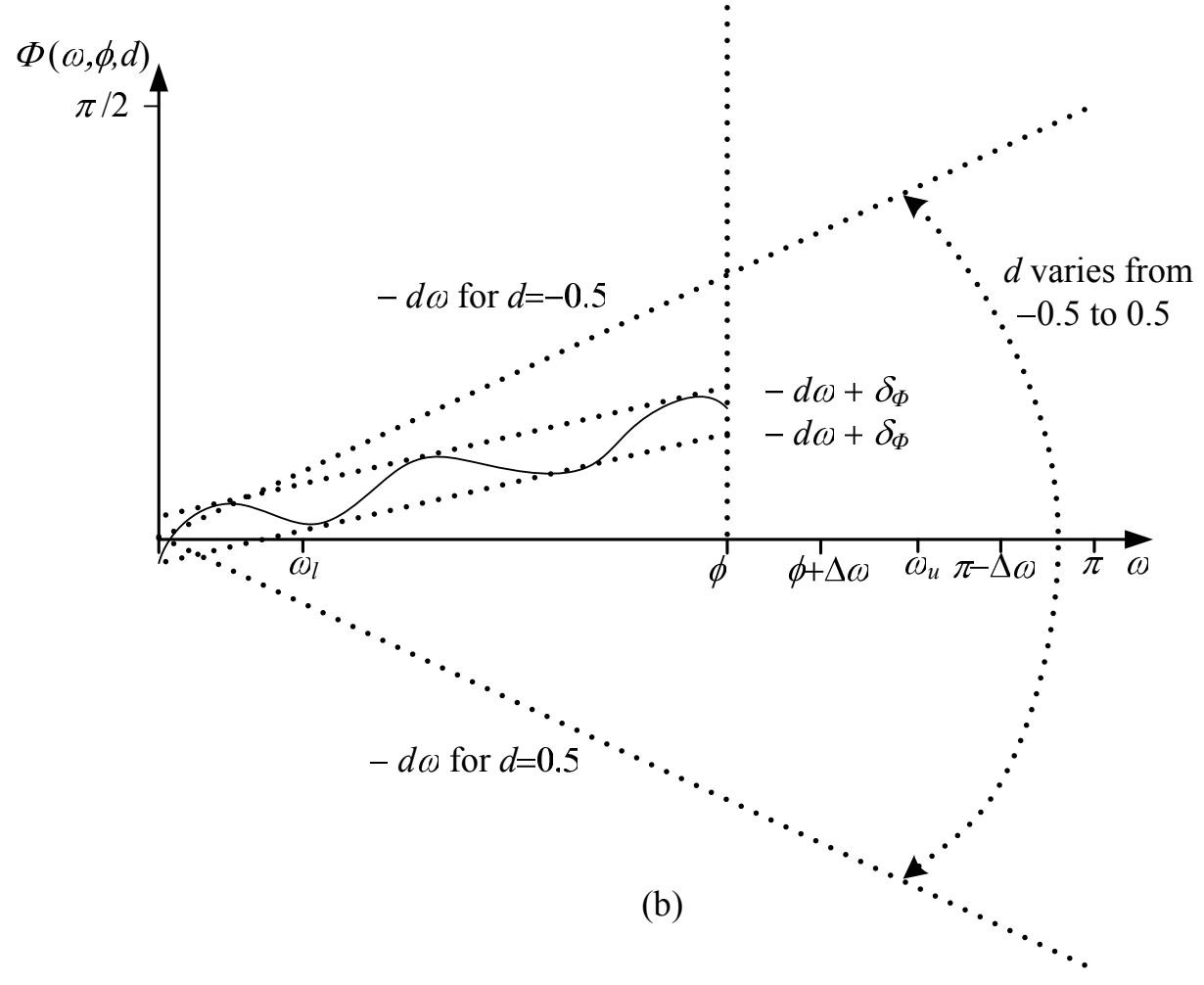

Figure 2.11. Specifications for (a) magnitude response and (b) phase response of a lowpass filter with variable passband edge $\phi$ and variable fractional delay $d$. 


\subsection{Design of Variable Bandedge and Fractional Delay Filters}

Deng [89] for the first time proposed a WLS method for the design of VBFD filters. In

this method, the $k$ th filter coefficient $h_{k}(\phi, d)$ is represented as a 2-D polynomial of the variables bandedge $\phi$ and fractional delay $d$ independently, given as

$$
h_{k}(\phi, d)=\sum_{p=0}^{P} \sum_{q=0}^{Q} a(k, p, q) \phi^{p} d^{q},
$$

where $P$ and $Q$ stand for the polynomial orders of the variables $\phi$ and $d$, respectively.

Hence, for a $K$ th order VBFD filter, the $z$-transform transfer function of the VBFD filter is obtained as

$$
\begin{aligned}
H(z, \phi, d) & =\sum_{k=-K / 2}^{K / 2} h_{k}(\phi, d) z^{-k} \\
& =\sum_{k=-K / 2}^{K / 2} \sum_{p=0}^{P} \sum_{q=0}^{Q} a(k, p, q) \phi^{p} d^{q} z^{-k} \\
& =\sum_{p=0}^{P} \sum_{q=0}^{Q} \sum_{k=-K / 2}^{K / 2} a(k, p, q) z^{-k} \phi^{p} d^{q} \\
& =\sum_{p=0}^{P} \sum_{q=0}^{Q} H_{p q}(z) \phi^{p} d^{q} .
\end{aligned}
$$

To implement the 2-D polynomial representations, a parallel structure was proposed in $[89,90]$ to update the filter coefficients in a fast manner. The parallel structure is shown in Figure 2.12.

To reduce the implementation complexity, (2.49) can be rewritten as

$$
\begin{aligned}
H(z, \phi, d) & =\sum_{p=0}^{P} \sum_{q=0}^{Q} H_{p q}(z) \phi^{p} d^{q} \\
& =\sum_{p=0}^{P} G_{p}(z, d) \phi^{p}
\end{aligned}
$$

where $G_{p}(z, d)$ is given as

$$
G_{p}(z, d)=\sum_{q=0}^{Q} H_{p q}(z) d^{q}
$$




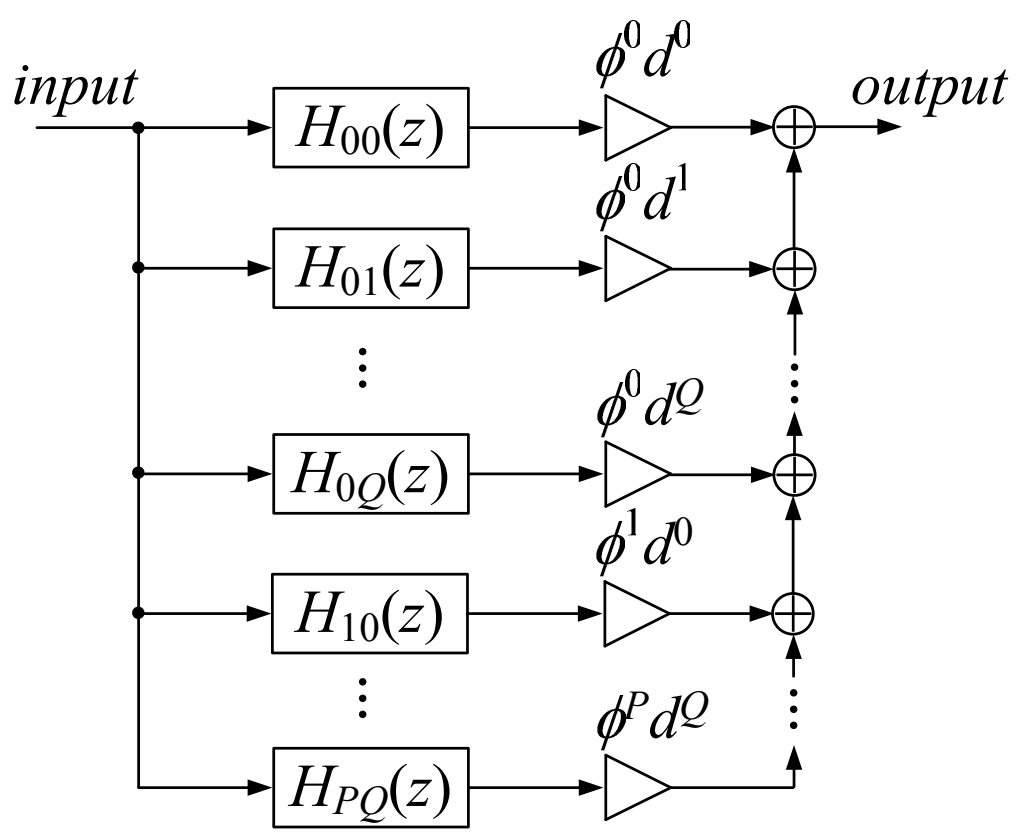

Figure 2.12. The parallel implementation of VBFD filters.

Thus, $H(z, \phi, d)$ can be implemented by a nested Farrow structure proposed in [91]. The implementation of $H(z, \phi, d)$ and $G_{p}(z, d)$ are shown in Figures 2.13(a) and (b), respectively. In this nested Farrow structure, each subfilter in the original Farrow structure was implemented by a sub Farrow structure. Compared with the parallel structure, although the nested Farrow structure has reduced the computational and implementation complexities significantly, it still requires $(K / 2+1)(P+1)(Q+1)$ multiplications per sample output.

A further improvement to the 2-D polynomials approximation of VBFD is proposed in [92], where the subfilter $H_{p q}(z)$ in (2.50) is separated into the cascade of two subfilters, given as

$$
H_{p q}(z)=U_{p}(z) V_{q}(z)
$$

Hence, (2.50) can be rewritten as

$$
H(z, \phi, d)=\sum_{p=0}^{P} \sum_{q=0}^{Q} U_{p}(z) V_{q}(z) \phi^{p} d^{q}=\sum_{p=0}^{P} U_{p}(z) V(z, d) \phi^{p}
$$




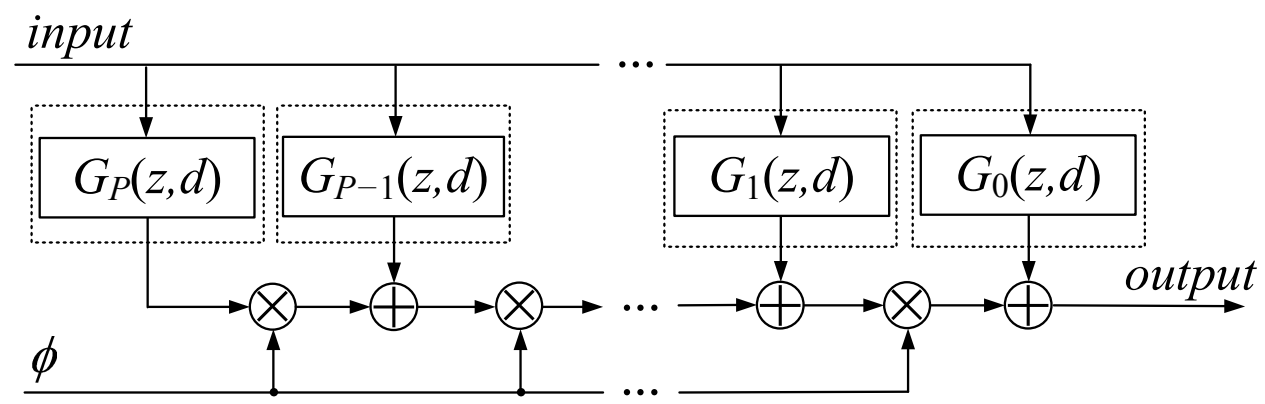

(a) The nested Farrow structure where each subfilter $G p(z, d)$ is implemented by a sub Farrow structure shown in Figure 2.13(b).

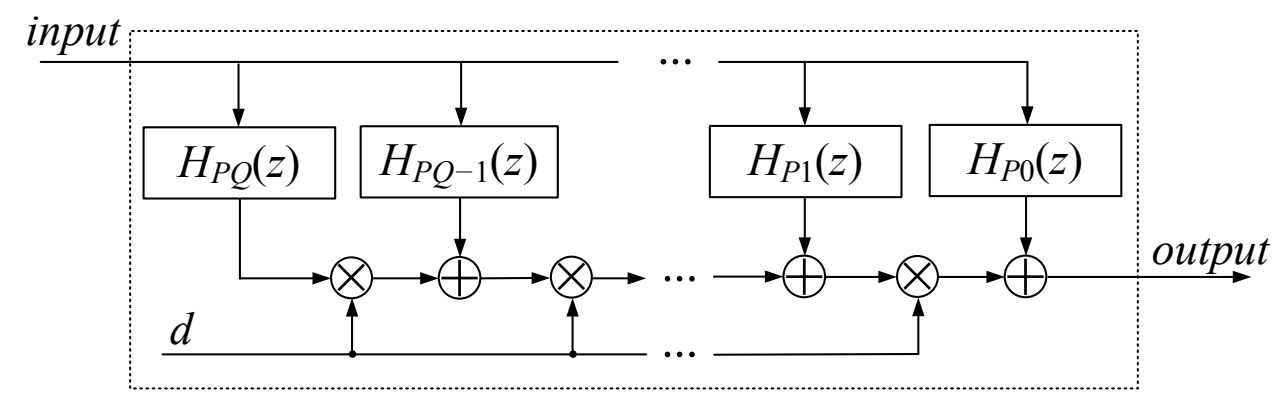

(b) The Farrow module $G_{P}(z, d)$.

Figure 2.13. The nested Farrow structure for the implementation of VBFD filters where $V(z, d)$ is given as

$$
V(z, d)=\sum_{q=0}^{Q} V_{q}(z) d^{q} .
$$

With the common prefilter $U_{p}(z)$ for each sub Farrow structure, the overall computational complexity is reduced. The structure is illustrated in Figure 2.14.

\subsection{Frequency Response Masking and Fast Filter Bank}

As reviewed in Section 2.2, the technique proposed in [31] makes use of frequency response masking (FRM) and fast filter bank (FFB) for the design of variable bandedge filters with very shape transition band. Since the FRM and FFB techniques will also be used in the design of VBFD proposed in Chapters 5 and 6, the review of both two techniques is given in this chapter. 


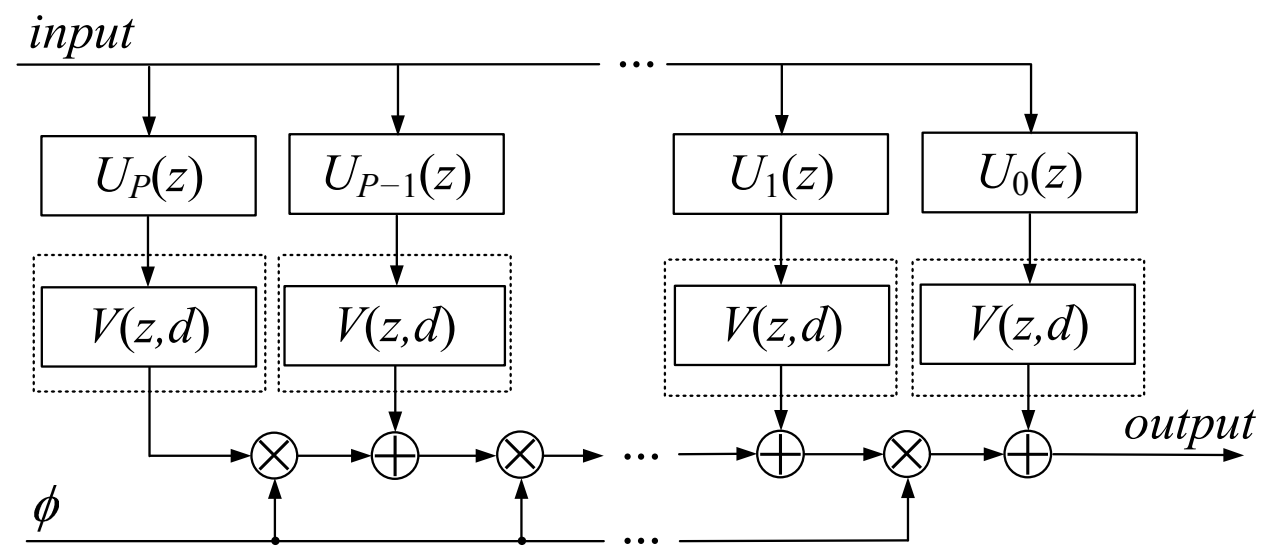

(a) The first level $p$ th subfilter $V(z, d)$ in the Farrow structure is cascaded with a filter $U_{p}(z)$.

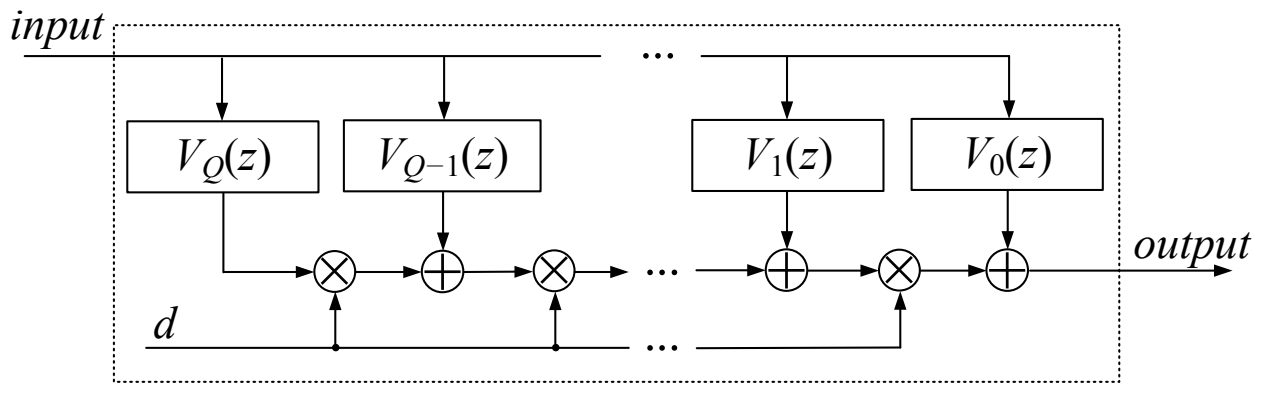

(b) The Farrow module $V(z, d)$ in Figure 2.14(a).

Figure 2.14. Implementation structure for VBFD filters proposed in [91]

\subsubsection{Frequency Response Masking}

The FRM technique was proposed in [120] for the design of linear-phase FIR filters with very sharp transition width and low computational complexity. The structure of the FRM is shown in Figure 2.15. In this structure, the filter $F_{a}\left(e^{j \omega}\right)$ is the frequency compressed version of a prototype bandedge shaping filter $F\left(e^{j \omega}\right)$ obtained by replacing each delay of $F\left(e^{j \omega}\right)$ with $L$ delays. $F\left(e^{j \omega}\right)$ and $F_{a}\left(e^{j \omega}\right)$ are shown in Figures 2.16(a) and (b), respectively. The filter $F_{c}\left(e^{j \omega}\right)$, the complement of $F_{a}\left(e^{j \omega}\right)$, is obtained by subtracting $F_{a}\left(e^{j \omega}\right)$ from a pure delay, $e^{-j L N_{F} \omega}$, where $N_{F}$ is the group delay of $F_{a}\left(e^{j \omega}\right)$. The transition bandwidths of $F_{a}\left(e^{j \omega}\right)$ and $F_{c}\left(e^{j \omega}\right)$ are shrunk by a factor $L$, compared with the prototype filter $F\left(e^{j \omega}\right)$. The outputs of the filters $F_{a}\left(e^{j \omega}\right)$ and $F_{c}\left(e^{j \omega}\right)$ are filtered by two 


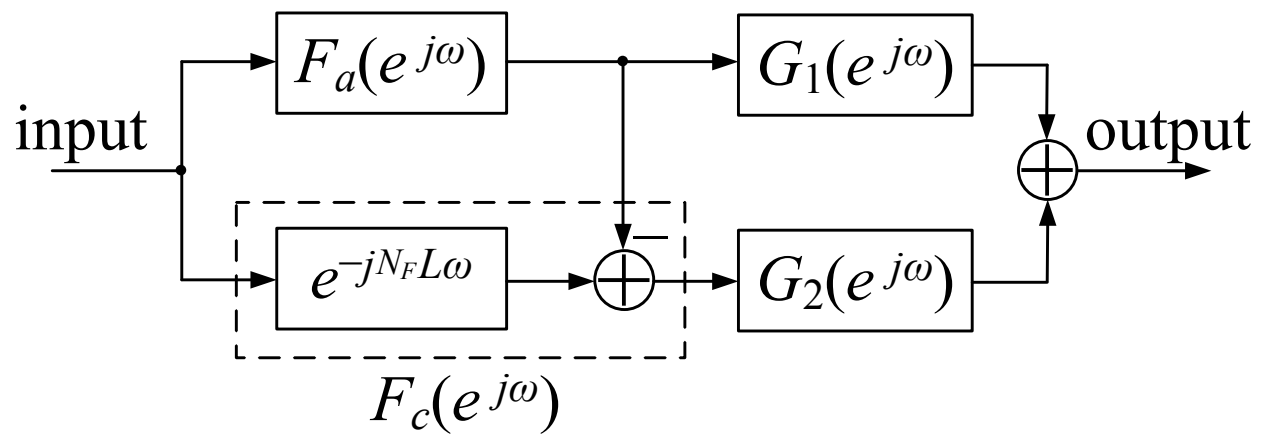

Figure 2.15. The structure of the frequency response masking filter.
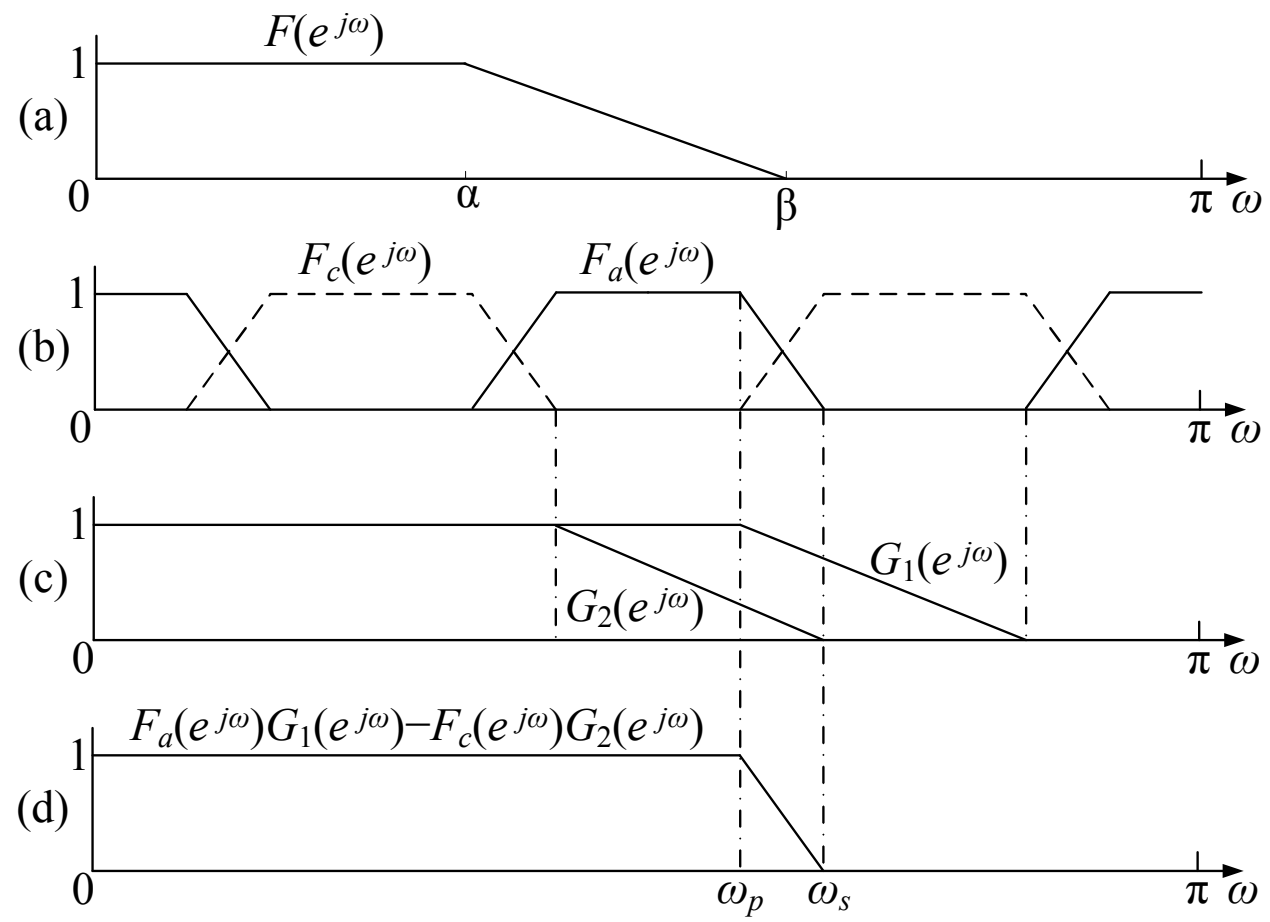

Figure 2.16. The frequency responses of FRM sub-filters.

masking filters $G_{1}\left(e^{j \omega}\right)$ and $G_{2}\left(e^{j \omega}\right)$, respectively, to retain the desired frequency bands. The frequency responses of the two masking filters are shown in Figure 2.16(c). By combining the retained bands of $F_{a}\left(e^{j \omega}\right)$ and $F_{c}\left(e^{j \omega}\right)$, the final frequency response has a very sharp transition band as shown in Figure 2.16(d). The three filters constructing the FRM structure, i.e., $F\left(e^{j \omega}\right), G_{1}\left(e^{j \omega}\right)$ and $G_{2}\left(e^{j \omega}\right)$, all have relatively wider transition bands than the resultant sharp transition width. Therefore, the overall computational complexity of the FRM structure is low. 


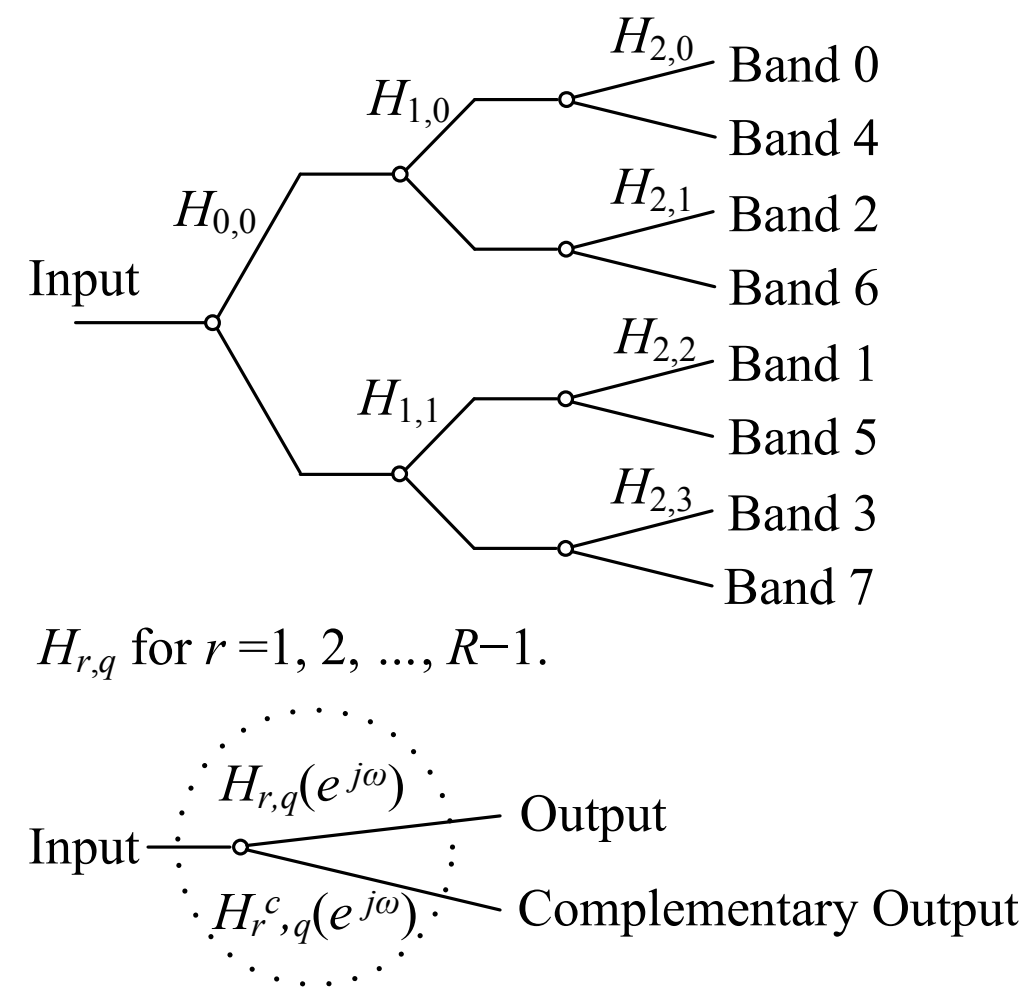

Figure 2.17. The structure of a 3-stage FFB.

\subsubsection{Fast Filter Bank}

The FFB [121] is a filter bank version of the FRM and it is also a generalized sliding fast Fourier transform (FFT). In the FFB, the compressed and shifted versions of the complementary first order prototype FIR filters in the butterfly operations of FFT, $1+z^{-1}$ and $1-z^{-1}$, are replaced by short complementary half band filters, such that the frequency selectivity of the resulting FFB is significantly improved with low complexity. Since it is a generalization of FFT, the original FFB is a multi-stage radix-2 filter bank, where the filters in each stage split each channel of signal further into 2 channels. In an $R$-stage FFB, the input signal is decomposed into $N\left(N=2^{R}\right)$ channels in the frequency range from dc to the sampling frequency. The structure of an eight-channel $(R=3)$ analysis FFB is shown in Figure 2.17.

In Figure 2.17, the filters $H_{r, q}\left(e^{j \omega}\right)$ for $r=0, \ldots, R-1$, is a frequency compressed 
and shifted version of a prototype lowpass half band filter $H_{r}\left(e^{j \omega}\right)$, given by

$$
H_{r, q}\left(e^{j \omega}\right)=H_{r}\left(e^{j\left(2^{R-1-r} \omega+\frac{2 \pi \tilde{q}}{N}\right)}\right)
$$

where $\tilde{q}$ is the bit-reversed version of $q$ in $R-1$ bits.

The filters $H_{r, q}\left(e^{j \omega}\right)$ and $H_{r, q}^{c}\left(e^{j \omega}\right)$ form a complementary pair, given by

$$
H_{r, q}\left(e^{j \omega}\right)+H_{r, q}^{c}\left(e^{j \omega}\right)=2 e^{-j 2^{R-1-r} N_{r} \omega}
$$

where $N_{r}$ is the group delay of the prototype filter $H_{r}\left(e^{j \omega}\right)$.

Figure 2.18 illustrates the frequency responses of the filter in each stage of the 8channel FFB that results in band 1. The frequency responses of the filters $H_{r, q}\left(e^{j \omega}\right)$ and $H_{r, q}^{c}\left(e^{j \omega}\right)$ for $r=0,1,2$, along the path to produce band 1, are shown in Figures 2.18(a), (b) and (d), respectively. Figure 2.18(c) shows the products of $H_{0,0}^{c}\left(e^{j \omega}\right)$ and $H_{1,1}\left(e^{j \omega}\right)$, and the resultant frequency response of band 1 is shown in Figure 2.18(e). Figure 2.18 shows, by shifting the frequency responses of each stage with different phases, different bands can be produced.

\subsection{Summary}

In this chapter, the concepts of phase delay, group delay and fractional delay are illustrated. In the meanwhile, the concepts of variable bandedge filters, variable fractional delay filters and variable bandedge and fractional delay filters are illustrated and their design approaches are reviewed. In addition, two popular applications of fractional delay filters are given. In the end, the techniques FRM and the FFB are reviewed. 
(a)

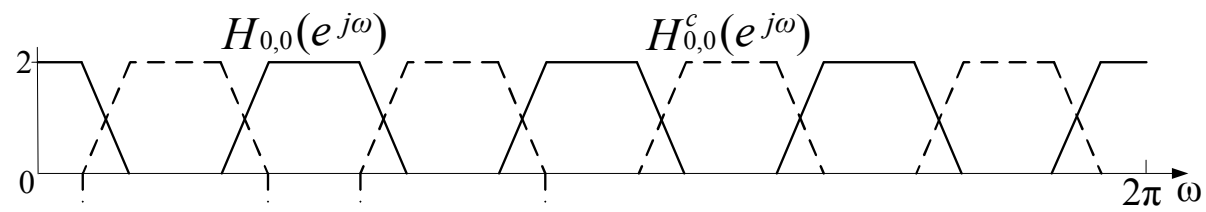

(b)

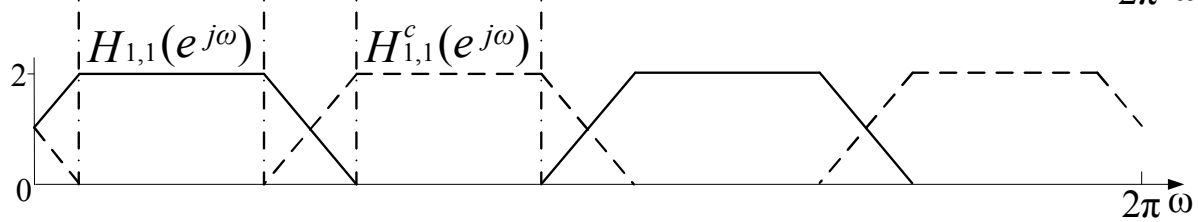

(c)

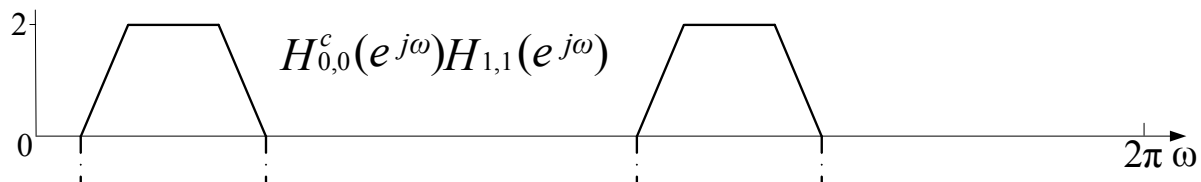

(d)

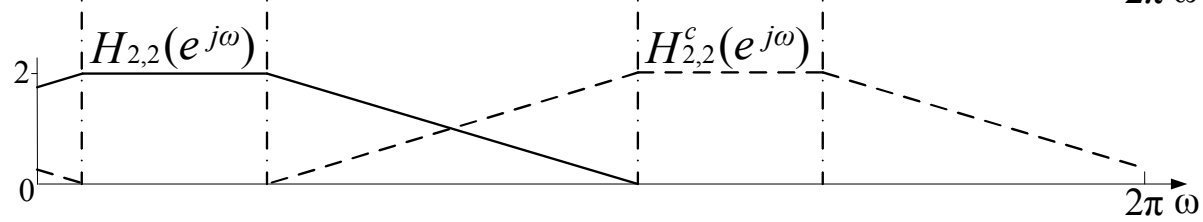

(e)

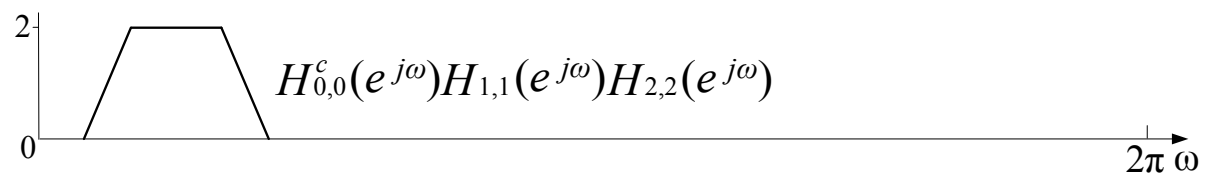

Figure 2.18. The frequency responses of the filters in an 8-channel FFB. 


\section{Chapter 3}

\section{Polynomial Implementation of Lagrange-type Variable Fractional Delay Filters}

As reviewed in Chapter 2, the Lagrange interpolation effectively synthesizes the variable fractional delay (VFD) when it is implemented in the Farrow structure [48]. In this chapter, a polynomial implementation structure is proposed for the Lagrange-type VFD filters, saving the implementation complexity nearly by half. The chapter is organized as follows. The conventional Farrow structure implementations of the Lagrange-type VFD filters are reviewed in Section 3.1. Section 3.2 investigates the coefficient matrix of the Farrow structure based Lagrange-type VFD filters and 3 new properties of the matrix are investigated. Based on these properties, the efficient polynomial implementation of the Lagrange-type VFD filters is proposed in Chapter 3.3. Numerical examples are presented in Chapter 3.4.

\subsection{The Farrow Structure Based Lagrange-Type Vari- able Fractional Delay Filters}

In the Lagrange interpolation, the order of the VFD filter $N$ is the same as $M$, the order of the polynomial for the approximation of each coefficient. The $z$-transform transfer 
function of the VFD filters in (2.30) is enforced to satisfy

$$
\sum_{n=0}^{N} C_{n}(z) D^{n}=z^{-D}
$$

for $D=0, \ldots, N$. The matrix equation (3.1) can be implemented in the Farrow structure as shown in Figure 2.10, where $C_{n}(z)$ is the $z$-transform transfer function of the $n$th subfilter.

The fractional delays other than $0,1, \ldots, N$ are interpolated from those integer delays; for this reason, the technique is named as an interpolation approach. Represented in matrix form, (3.1) can be written to

$$
\mathbf{V C}=\mathbf{z}
$$

where $\mathbf{C}=\left[C_{0}(z), C_{1}(z), \ldots, C_{N}(z)\right]^{T}, \mathbf{z}=\left[1, z^{-1}, \ldots, z^{-N}\right]^{T}$ is a delay vector, and $\mathbf{V}$ is the Vandermonde matrix $\mathbf{V}$, given as

$$
\mathbf{V}=\left(\begin{array}{ccccc}
1 & 0 & 0 & \cdots & 0 \\
1 & 1 & 1 & \cdots & 1 \\
1 & 2 & 4 & \cdots & 2^{N} \\
\cdots & \cdots & \cdots & \cdots & \cdots \\
1 & N & N^{2} & \cdots & N^{N}
\end{array}\right)
$$

Thus (3.2) can be rewritten as

$$
\mathbf{C}=\mathbf{Q z}
$$

where $\mathbf{Q}=(\mathbf{V})^{-1}$ is the coefficient matrix of the Lagrange-type VFD filters, i.e., the $i$ th row of $\mathbf{Q}$ is the impulse response of the $i$ th subfilter in the Farrow structure.

It is well known that the implementation complexity of the Farrow structure based Lagrange-type VFD filters is high, i.e., for an $N$ th order interpolation, $N+1$ subfilters of order $N$ are synthesized and $(N+1)^{2}$ multiplications and $N^{2}+2 N-1$ additions 
are required per sample. In [35], Deng proposed a transformation matrix $\mathbf{S}$, so that the transformed coefficient matrix $\widetilde{\mathbf{Q}}$

$$
\widetilde{\mathbf{Q}}=\mathbf{S Q}
$$

holds symmetric subfilter coefficients, where the $(i, j)$ th element of the $(N+1) \times(N+1)$ transformation matrix $\mathbf{S}$ is given by

$$
s_{i, j}=\left\{\begin{array}{cl}
\left(\frac{N}{2}\right)^{j-i}\left(\begin{array}{c}
j \\
i
\end{array}\right), & 0 \leq i \leq j \leq N \\
0, & \text { otherwise. }
\end{array}\right.
$$

The resulting $\widetilde{\mathbf{Q}}$ with element $\widetilde{q_{i, j}}$ and the delay parameter $d$ satisfy

$$
\widetilde{q_{i, j}}=(-1)^{i} \widetilde{q_{i, N-j}}
$$

and

$$
d=D-\frac{N}{2}
$$

Thus the matrix equation in (3.4) becomes

$$
\widetilde{\mathbf{C}}=\widetilde{\mathbf{Q}} \mathbf{z}
$$

and hence the $z$-transform transfer function of the VFD filters in (3.1) is changed to

$$
\sum_{n=0}^{N} \widetilde{C}_{n}(z) d^{n}=z^{-d}
$$

where $\widetilde{C}_{n}(z)$ is the $n$th row vector of $\widetilde{\mathbf{C}}$, given as

$$
\widetilde{C}_{n}(z)=\sum_{j=0}^{N} \widetilde{q_{n, j}} z^{-j}
$$

From (3.7) and (3.11), it can be found that the transformed subfilter $\widetilde{C}_{n}(z)$ is symmetric for even $n$ and antisymmetric for odd $n$. Such symmetry does not exist in the original coefficient matrix. Hence the even-odd structure [34] as shown in Figure 3.1 is used for the Lagrange interpolation, reducing the implementation complexity by half compared with the original coefficient matrix implemented in the Farrow structure. 


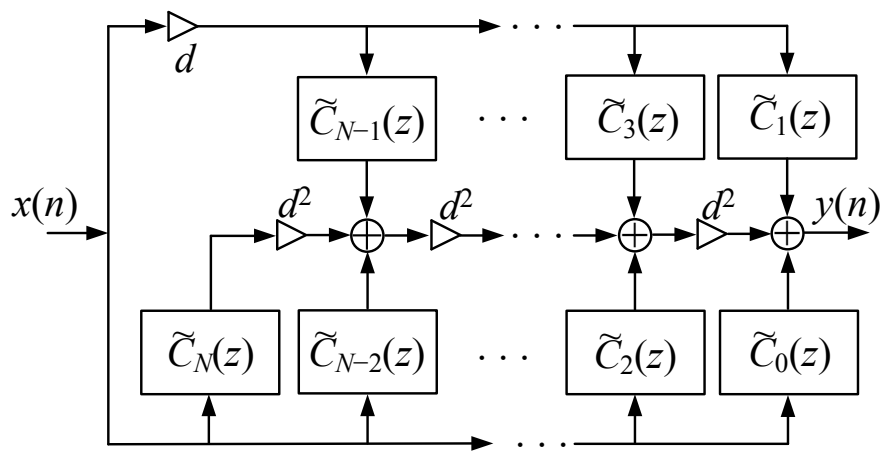

(a)

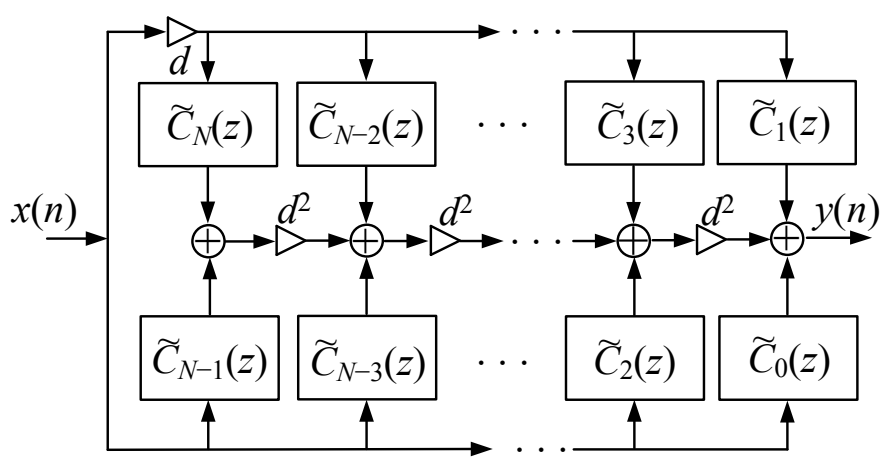

(b)

Figure 3.1. The even-odd structure for the Lagrange interpolation for (a) even $N$ and (b) odd $N$, respectively.

Based on the above transform technique, in the following of this chapter, 3 new properties of the subfilter coefficient matrix $\widetilde{\mathbf{Q}}$ are discovered. These properties can be employed to reduce the implementation complexity further.

\subsection{Properties of the Transformed Coefficient Matrix}

Property 1: For even $N$, the coefficients of the $(2 i-1)$ th subfilter, i.e., the $(2 i-1)$ th row of $\widetilde{\mathbf{Q}}$, are related to those of the $2 i$ th subfilter, i.e., the $2 i$ th row of $\widetilde{\mathbf{Q}}$, by,

$$
\frac{\widetilde{q_{2 i-1, j}}}{\widetilde{q_{2 i, j}}}=j-\frac{N}{2}, \text { for } i=1, \ldots, \frac{N}{2} \text { and } j=0, \ldots, N \text {. }
$$

Proof: The coefficient matrix $\mathbf{Q}$, i.e., the inverse of the Vandermonde matrix $\mathbf{V}$, can be decomposed into an upper triangular matrix $\mathbf{U}$ and a lower triangular matrix $\mathbf{L}$ [128], as

$$
\mathbf{Q}=\mathbf{U L}
$$


The $(i, j)$ th element of the matrices $\mathbf{U}$ and $\mathbf{L}$, denoted as $u_{i, j}$ and $l_{i, j}$, are expressed as (3.13) and (3.14)

$$
\begin{gathered}
u_{i, j}=(-1)^{i+j} \sigma_{j-i}(0,1, \ldots, j), \text { for } 0 \leq i \leq j \leq N, \\
l_{i, j}=\left(\prod_{k=0 \text { and } k \neq j}^{i} j-k\right)^{-1}, \text { for } 0 \leq i \leq j \leq N
\end{gathered}
$$

respectively. In (3.13), $\sigma_{k}\left(x_{1}, \ldots, x_{r}\right)$ denotes the elementary symmetric function of degree $k$ in $r$ variables [129], given by

$$
\sigma_{k}\left(x_{1}, \ldots, x_{r}\right)=\sum_{1 \leq p_{1}<p_{2}<\cdots<p_{k} \leq r} x_{p_{1}} x_{p_{2}} \cdots x_{p_{k}}, 0 \leq k \leq r
$$

i.e., $\sigma_{k}\left(x_{1}, \ldots, x_{r}\right)$ is the sum of all products of $k$-tuples of the $r$ variables. For $k=0$, $\sigma_{0}\left(x_{1}, \ldots, x_{r}\right)$ is defined to be 1 .

According to (3.12)-(3.14) and (3.5)-(3.6), $\widetilde{q_{i, j}}$ is given by

$$
\widetilde{q_{i, j}}=\sum_{k=i}^{N}\left(\frac{N}{2}\right)^{k-i}\left(\begin{array}{l}
k \\
i
\end{array}\right) \frac{(-1)^{N-k} \sigma_{N-k}(0, \ldots, j-1, j+1, \ldots, N)}{\prod_{p=0 \text { and } p \neq j}^{N} j-p} .
$$

And the $j$ th coefficient of the overall Lagrange-type VFD filter, $\widetilde{h(j, d)}$, is given by,

$$
\widetilde{h(j, d)}=\sum_{k=0}^{N} \widetilde{q_{k, j}} d^{k}, \text { for } j=0, \ldots, N
$$

i.e., $\widetilde{h(j, d)}$ is an $N$ th order polynomial of $d$, where the coefficient of the $p$ th power term of $d$ is corresponding to the element $\widetilde{q_{p, j}}$ of the transformed coefficient matrix $\widetilde{\mathbf{Q}}$. 
Substituting (3.16) into (3.17), (3.17) can be rewritten as

$$
\begin{aligned}
\widetilde{h(j, d)} & =\sum_{i=0}^{N} d^{i} \sum_{k=i}^{N}\left(\frac{N}{2}\right)^{k-i}\left(\begin{array}{c}
k \\
i
\end{array}\right) \frac{(-1)^{N-k} \sigma_{N-k}(0,1, \ldots, j-1, j+1, \ldots, N)}{\prod_{p=0 \text { and } p \neq j}^{N} j-p} \\
& =\frac{\sum_{k=0}^{N} \sum_{i=0}^{N-k}(-1)^{k}\left(\begin{array}{c}
N-k \\
i
\end{array}\right)\left(\frac{N}{2}\right)^{N-k-i} d^{i} \sigma_{k}(0,1, \ldots, j-1, j+1, \ldots, N)}{\prod_{p=0 \text { and } p \neq j}^{N} j-p} \\
= & \frac{\sum_{k=0}^{N}(-1)^{k}\left(d+\frac{N}{2}\right)^{N-k} \sigma_{k}(0,1, \ldots, j-1, j+1, \ldots, N)}{\prod_{p=0}^{N} j-p} \\
= & \frac{\left(d+\frac{N}{2}\right)^{N} \sum_{k=0}^{N}\left(-\frac{1}{d+\frac{N}{2}}\right)^{k} \sigma_{k}(0,1, \ldots, j-1, j+1, \ldots, N)}{\prod_{p=0}^{N} \text { and } p \neq j_{j} j-p}
\end{aligned}
$$

According to the generating function of the elementary symmetric function given by [129],

(3.18) is simplified as,

$$
\sum_{k \geq 0} \sigma_{k}\left(x_{1}, x_{2}, \ldots, x_{r}\right) q^{k}=\prod_{1 \leq i \leq r}\left(1+x_{i} q\right)
$$

$$
\begin{aligned}
\widetilde{h(j, d)} & =\prod_{p=0 \text { and } p \neq j}^{N} \frac{d+\frac{N}{2}-p}{j-p} \\
& =\frac{(-1)^{j}\left(d+j-\frac{N}{2}\right)}{(N-j) ! j ! d} \prod_{p=0 \text { and } p \neq\left|\frac{N}{2}-j\right|}^{\frac{N}{2}}\left(d^{2}-p^{2}\right) .
\end{aligned}
$$

Let

$$
G(j, d)=\prod_{p=0 \text { and } p \neq\left|\frac{N}{2}-j\right|}^{\frac{N}{2}}\left(d^{2}-p^{2}\right)=\sum_{i=0}^{\frac{N}{2}} t_{i, j} d^{2 i},
$$

where $t_{i, j}$ is the coefficient of the $2 i$ th power term of $d$ in $G(j, d)$. It is evident that

$$
G(j, d)=G(N-j, d), \text { for } j=0, \ldots, N
$$

Substituting (3.21) into (3.22), it can be found that

$$
t_{i, j}=t_{i, N-j} .
$$


From (3.20), it can be found that the coefficient of the zero-th power term of $d$ is 1 for $j=\frac{N}{2}$ and 0 for $j \neq \frac{N}{2}$, i.e.,

$$
\widetilde{q_{0, j}}=\left\{\begin{array}{l}
1 j=\frac{N}{2}, \\
0 \text { otherwise. }
\end{array}\right.
$$

Furthermore, from (3.17), (3.20) and (3.21), it cab be found that

$$
\widetilde{q_{2 i-1, j}}=(-1)^{j} \frac{\left(j-\frac{N}{2}\right) t_{i, j}}{N !}\left(\begin{array}{c}
N \\
j
\end{array}\right) \text { and } \widetilde{q_{2 i, j}}=(-1)^{j} \frac{t_{i, j}}{N !}\left(\begin{array}{c}
N \\
j
\end{array}\right) \text {. }
$$

Therefore,

$$
\widetilde{\widetilde{q_{2 i-1, j}}}=j-\frac{N}{2}, \text { for } i=1, \ldots, \frac{N}{2} \text { and } j=0, \ldots, N \text {. }
$$

Property 2: For odd $N$, the coefficients of the $2 i$ th subfilter, i.e., the $2 i$ th row of $\widetilde{\mathbf{Q}}$, are related to those of the $(2 i+1)$ th subfilter, i.e., the $(2 i+1)$ th row of $\widetilde{\mathbf{Q}}$, by

$$
\frac{\widetilde{q_{2 i, j}}}{\widetilde{q_{2 i+1, j}}}=j-\frac{N}{2}, \text { for } i=0, \ldots, \frac{N-1}{2} \text { and } j=0, \ldots, N \text {. }
$$

The proof of Property 2 is similar to that of Property 1 and is omitted.

Property 3: $c \widetilde{\mathbf{Q}}$ is an integer matrix, where $c=N$ ! for even $N$ and $c=2^{N} N$ ! for $\operatorname{odd} N$.

Proof: According to the generating function of the elementary symmetric function in (3.19), $G(j, d)$ in (3.21) can be rewritten as

$G(j, d)=d^{N} \prod_{p=0 \text { and } p \neq\left|\frac{N}{2}-j\right|}^{\frac{N}{2}}\left(1-\frac{p^{2}}{d^{2}}\right)=d^{N} \sum_{i=0}^{\frac{N}{2}} \sigma_{i} d^{-2 i}=\sum_{i=0}^{\frac{N}{2}} \sigma_{i} d^{N-2 i}=\sum_{i=0}^{\frac{N}{2}} \sigma_{\frac{N}{2}-i} d^{2 i}$.

Note: $\sigma_{i}$ in (3.26) is an abbreviation of

$$
\sigma_{i}\left(0, \ldots,-\left(\frac{N}{2}-j-1\right)^{2},-\left(\frac{N}{2}-j+1\right)^{2}, \ldots,-\left(\frac{N}{2}\right)^{2}\right) .
$$

Comparing (3.26) with (3.21), it can be obtained that

$$
t_{i, j}=\sigma_{\frac{N}{2}-i}\left(0, \ldots,-\left(\frac{N}{2}-j-1\right)^{2},-\left(\frac{N}{2}-j+1\right)^{2}, \ldots,-\left(\frac{N}{2}\right)^{2}\right) .
$$


Moreover, for even $N$, the variables of the elementary symmetric function in (3.27) are all integers. Therefore, according to the definition of the elementary symmetric function, $t_{i, j}$ are all integers, for $i=1, \ldots, \frac{N}{2}$ and $j=0, \ldots, N$. In addition, the subfilter coefficient values $\widetilde{q_{2 i-1, j}}$ and $\widetilde{q_{2 i, j}}$ are expressed in terms of $t_{i, j}$ as shown in (3.25). According to the binomial property, it can be seen that, scaled by a common factor of $c=N !, \widetilde{q_{i, j}}$ are all integers for $i=1, \ldots, N$ and $j=0, \ldots, N$. Combined with (3.24), Property 3 for even $N$ is proved. Similarly, for odd $N, \widetilde{q_{i, j}}$ are all integers if they are scaled by a factor $c=2^{N} N$ !.

Property 3 shows that, the coefficient matrix can be synthesized as an integer matrix scaled by a common factor $c$. Hence no quantization errors are introduced when the filter coefficients are expressed in $M$ bits for $M \geq\left\lceil\log _{2}(\max (|c \widetilde{\mathbf{Q}}|))\right\rceil$. In contrast, in the existing techniques, the filter coefficients are expressed in infinite precision form and the quantization errors may be introduced.

\subsection{Polynomial Implementation Strategy}

The properties discussed in Section 3.2 show the dependence of coefficient values between adjacent rows of $\widetilde{\mathbf{Q}}$. Therefore, besides the coefficient symmetry exploited in $[34,35,38]$, the dependencies of coefficient values are taken into consideration in this section to develop an efficient implementation strategy, resulting in further reduced complexities. The following subsections present the derivation of the polynomial structures for even and odd order Lagrange-type VFD filters, respectively.

\subsubsection{Polynomial Implementation for even order Lagrange-type VFD filters}

For even order Lagrange-type VFD filters, let $P_{j}(d)$ be an odd polynomial of $d$, given by

$$
P_{j}(d)=\sum_{i=1}^{\frac{N}{2}} p_{j}(2 i) d^{2 i-1}, \text { for } j=0, \ldots, \frac{N}{2} .
$$


where $p_{j}(2 i)=c \widetilde{q_{2 i, j}}$. Thus, according to Property 1 , the $j$ th coefficient of the overall Lagrange-type VFD is given by

$$
\widetilde{h(j, d)}=\widetilde{q_{0, j}}+\sum_{i=1}^{\frac{N}{2}} \widetilde{q_{2 i-1, j}} d^{2 i-1}+\sum_{i=1}^{\frac{N}{2}} \widetilde{q_{2 i, j}} d^{2 i}=\widetilde{q_{0, j}}+\frac{P_{j}(d)}{c}\left(d+j-\frac{N}{2}\right) .
$$

Due to the symmetry of the subfilter coefficients, the coefficient $h(\widetilde{N-j}, d)$ can be obtained as

$$
h(\widetilde{N-j}, d)=\widetilde{q_{0, j}}+\frac{P_{j}(d)}{c}\left(d-\left(j-\frac{N}{2}\right)\right) .
$$

Therefore, the $z$-transform transfer function of the Lagrange-type VFD filters is given by

$$
\begin{aligned}
& \widetilde{H(z)}=\sum_{j=0}^{N} \widetilde{h(j, d)} z^{-j} \\
& =\widetilde{h\left(\frac{N}{2}\right)} z^{-\frac{N}{2}}+\sum_{j=0}^{\frac{N}{2}-1} \widetilde{h(j, d)} z^{-j}+\sum_{j=0}^{\frac{N}{2}-1} h(\widetilde{N-j}, d) z^{-(N-j)} \\
& =\left(\widetilde{q_{0, \frac{N}{2}}}+\frac{1}{c} d P_{\frac{N}{2}}(d)\right) z^{-\frac{N}{2}}+\frac{1}{c} \sum_{j=0}^{\frac{N}{2}-1}\left(P_{j}(d)\left(d+j-\frac{N}{2}\right)\right) z^{-j} \\
& +\frac{1}{c} \sum_{j=0}^{\frac{N}{2}-1}\left(P_{j}(d)\left(d-\left(j-\frac{N}{2}\right)\right)\right) z^{-(N-j)} \\
& =z^{-\frac{N}{2}}+\frac{1}{c}\left(\sum_{j=0}^{\frac{N}{2}-1} P_{j}(d)\left(\frac{N}{2}-j\right)\left(z^{-(N-j)}-z^{-j}\right)\right) \\
& +\frac{1}{c} d\left(P_{\frac{N}{2}}(d) z^{-\frac{N}{2}}+\sum_{j=0}^{\frac{N}{2}-1} P_{j}(d)\left(z^{-(N-j)}+z^{-j}\right)\right) .
\end{aligned}
$$

Hence, the implementation structure is given in Figure 3.2.

In Figure 3.2, it can be seen that an even $N$ th order Lagrange-type VFD filter can be synthesized by implementing $\frac{N}{2}+1$ polynomials of $P_{j}(d)$ for $j=0, \ldots, \frac{N}{2}$. The number of coefficient multiplications used is $\left(\frac{N}{2}+1\right)\left(\frac{N}{2}+2\right)$, where $\frac{N}{2}\left(\frac{N}{2}+2\right)$ are constant multiplications while the others are general multiplications. Compared to the direct implementation using $(N+1) N+N$ multiplications and the implementation exploiting 


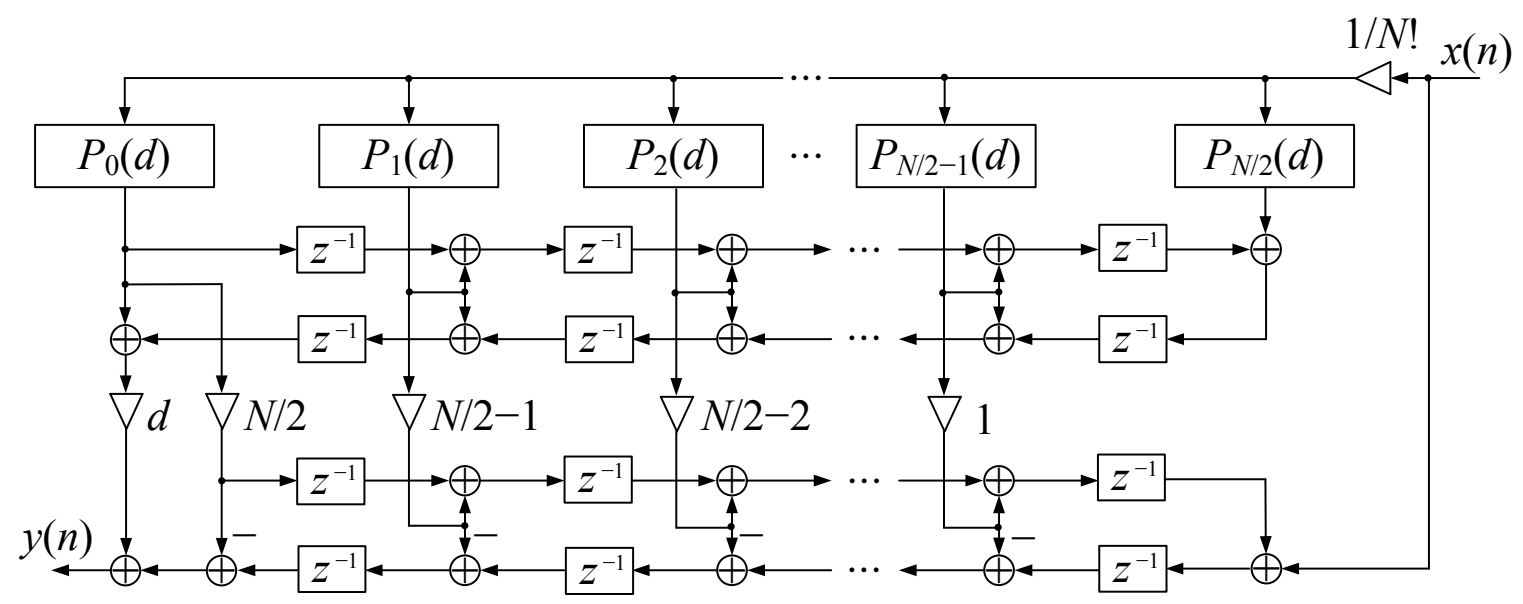

Figure 3.2. Polynomial structure for even $N$ th order Lagrange-type VFD filters. subfilter symmetry [34] using $(N+1) \frac{N}{2}+N$ multiplications, the proposed technique greatly reduces the computational complexity.

\subsubsection{Polynomial Implementation for odd order Lagrange-type VFD filters}

Similarly, for odd order Lagrange-type VFD filters, let $P_{j}(d)$ be an even polynomial of $d$, given by

$$
P_{j}(d)=\sum_{i=0}^{\frac{N-1}{2}} p_{j}(2 i+1) d^{2 i}, \text { for } j=0, \ldots, \frac{N-1}{2} .
$$

Thus, according to Property 2, the $j$ th coefficient of the overall Lagrange-type VFD is given by

$$
\widetilde{h(j, d)}=\sum_{i=0}^{\frac{N-1}{2}} \widetilde{q_{2 i, j}} d^{2 i}+\sum_{i=0}^{\frac{N-1}{2}} \widetilde{q_{2 i+1, j}} d^{2 i+1}=\frac{1}{c} P_{j}(d)\left(d+j-\frac{N}{2}\right) .
$$

Due to the symmetry of the subfilter coefficients, the coefficient $h(\widetilde{N-j}, d)$ is obtained as

$$
h(\widetilde{N-j}, d)=\frac{1}{c} P_{j}(d)\left(d-\left(j-\frac{N}{2}\right)\right) .
$$




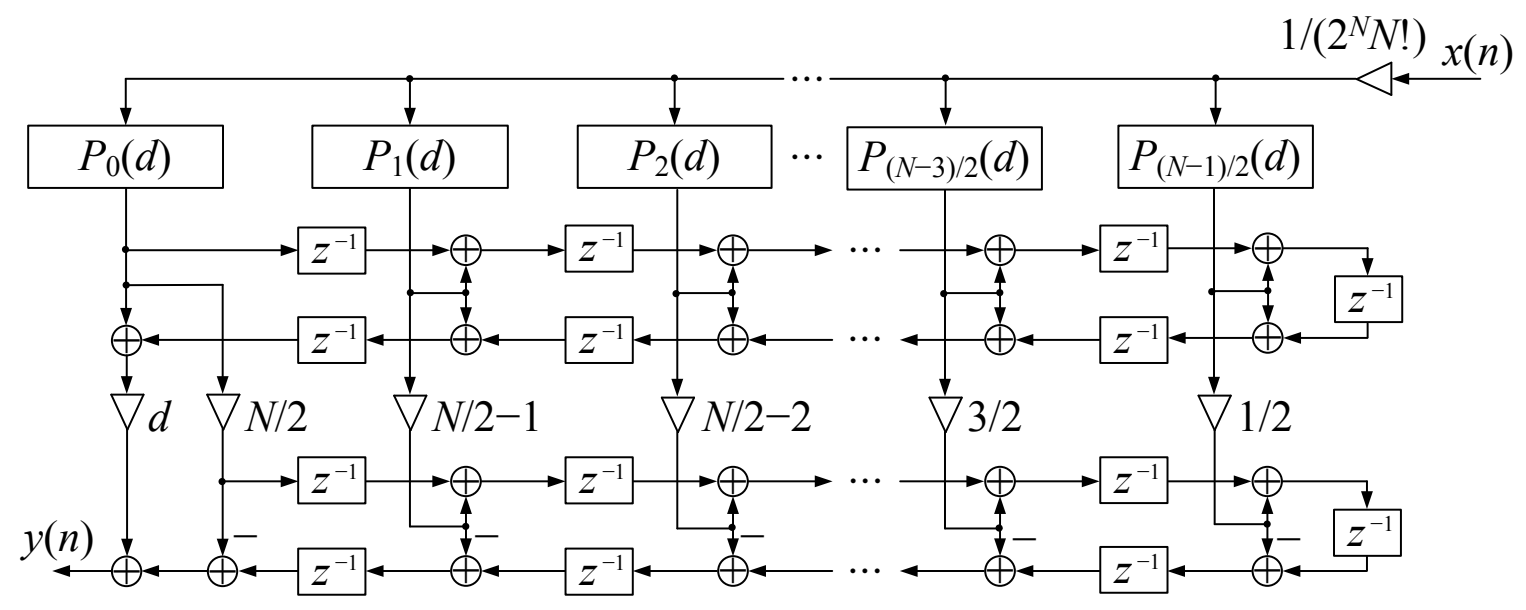

Figure 3.3. Polynomial structure for odd $N$ th order Lagrange-type VFD filters.

Therefore, the $z$-transform transfer function of the Lagrange-type VFD filters is given by

$$
\begin{aligned}
\widetilde{H(z)} & =\sum_{j=0}^{N} \widetilde{h(j, d)} z^{-j} \\
& \left.=\sum_{j=0}^{\frac{N-1}{2}} \widetilde{h(j, d)} z^{-j}+\sum_{j=0}^{\frac{N-1}{2}} \widehat{h(N-j}, d\right) z^{-(N-j)} \\
& =\frac{1}{c} \sum_{j=0}^{\frac{N-1}{2}}\left(P_{j}(d)\left(d+j-\frac{N}{2}\right)\right) z^{-j}+\frac{1}{c} \sum_{j=0}^{\frac{N-1}{2}}\left(P_{j}(d)\left(d-\left(j-\frac{N}{2}\right)\right)\right) z^{-(N-j)} \\
& =\frac{1}{c}\left(\sum_{j=0}^{\frac{N-1}{2}} P_{j}(d)\left(\frac{N}{2}-j\right)\left(z^{-(N-j)}-z^{-j}\right)\right) \\
& +\frac{d}{c} \sum_{j=0}^{\frac{N-1}{2}}\left(P_{j}(d)\left(z^{-(N-j)}+z^{-j}\right)\right) .
\end{aligned}
$$

Hence, the implementation structure is given in Figure 3.3.

Using the proposed technique, an odd $N$ th order Lagrange-type VFD filter can be synthesized by implementing $\frac{N+1}{2}$ polynomials of $P_{j}(d)$ for $j=0, \ldots, \frac{N-1}{2}$. The number of coefficient multiplications used is $\frac{N+1}{2} \frac{N+5}{2}+2$, where $\frac{N+1}{2} \frac{N+3}{2}+1$ are constant multiplications while the others are general multiplications. Compared with the direct implementation using $(N+1)^{2}+N$ multiplications and the implementation exploiting subfilter symmetry [34] using $\frac{(N+1)^{2}}{2}+N$ multiplications, the proposed implementation structure almost reduces the computational complexity by $3 / 4$ and $1 / 2$, respectively. 


\subsection{Numerical Examples}

In this section, a 5th and a 6th order Lagrange-type VFD filters are implemented using the proposed technique. The results are compared with that of [34], which achieves the lowest design cost for all existing Lagrange-type VFD filter techniques [33-35, 38-43].

\subsubsection{Implementation of a 5th order Lagrange-type VFD filter}

For a 5th order Lagrange-type VFD filter, the coefficient matrix according to (3.16) is given as

$$
\widetilde{\mathbf{Q}}=\frac{1}{3840}\left(\begin{array}{rrrrrrr}
45 & -375 & 2250 & 2250 & -375 & 45 \\
-18 & 250 & -4500 & 4500 & -250 & 18 \\
-200 & 1560 & -1360 & -1360 & 1560 & -200 \\
80 & -1040 & 2720 & -2720 & 1040 & -80 \\
80 & -240 & 160 & 160 & -240 & 80 \\
-32 & 160 & -320 & 320 & -160 & 32
\end{array}\right)
$$

Scaled by a factor $c=3840, c \widetilde{\mathbf{Q}}$ is an integer matrix. From (3.32), the 3 polynomials to be synthesized are:

$$
\begin{aligned}
& P_{0}(d)=-18+80 d^{2}+32 d^{4}, \\
& P_{1}(d)=250-1040 d^{2}+160 d^{4}, \\
& P_{2}(d)=-4500+2720 d^{2}-320 d^{4} .
\end{aligned}
$$

The implementation structure of the polynomials is illustrated in Figure 3.4 inside the dash box. Together with the remaining structures, the 5th order Lagrange-type VFD is implemented.

From Figure 3.4, it can be seen that in total 17 multiplications are required for each output sample. In comparison, the numbers of multiplications required in the direct implementation and the implementation exploiting the subfilter symmetry [34] are 41 and 


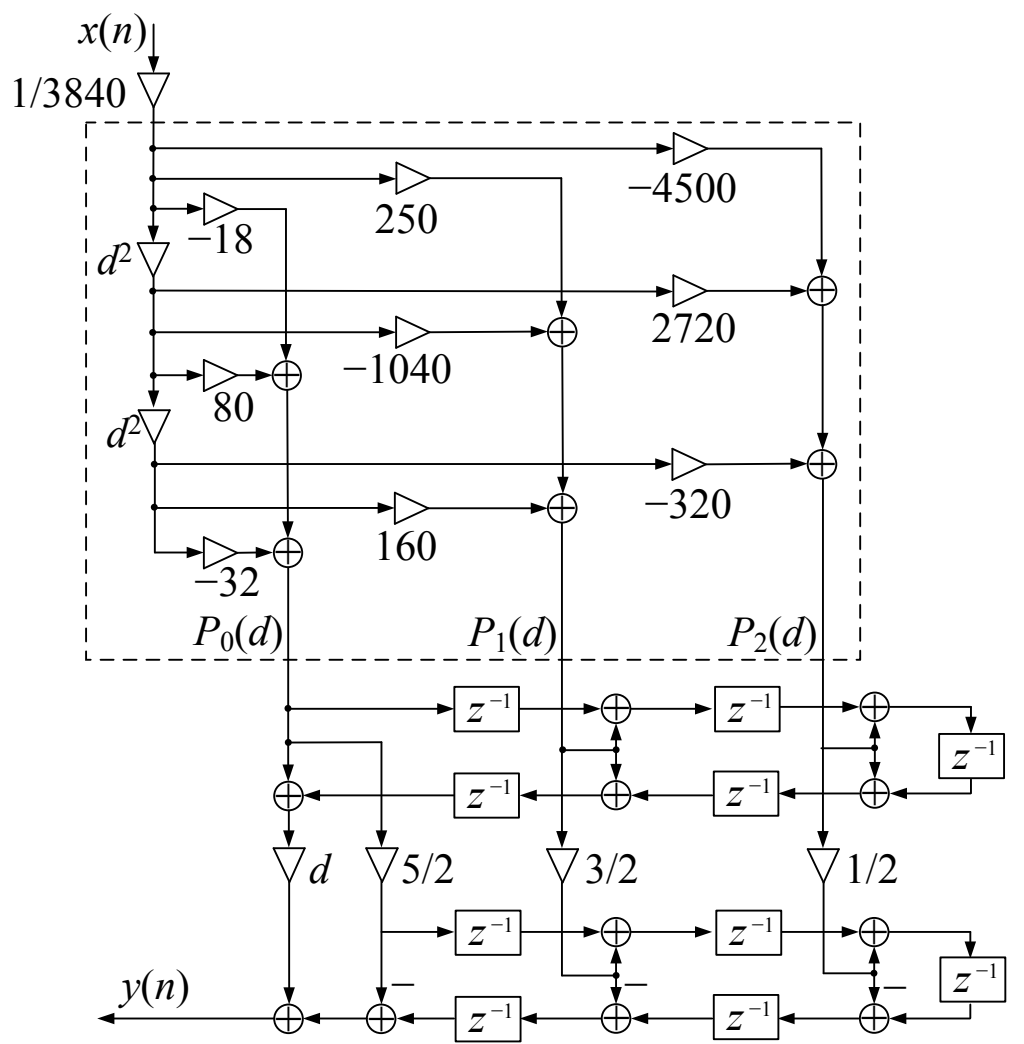

Figure 3.4. The implementation structure of the polynomials (in dash box) as well as the 5th order Lagrange-type VFD filter.

23 , respectively.

In the proposed implementation, since all the subfilter coefficients are integers after scaling, no quantization is introduced when the filter coefficients are expressed in 13 or more bit binary numbers. However, for the technique in [34], the filter coefficients are expressed in infinite precision and hence the quantization errors may be introduced. Figures 3.5(a), (b) and (c) illustrate the frequency response, magnitude and phase errors of the $5^{t h}$-order Lagrange-type VFD filters implemented using the proposed technique and the technique in [34] for $d=-0.3$ with 12-, 13- and 14-bit precisions, respectively.

When all the filter coefficients are represented in 13 or 14 bits, Figure 3.5 shows that the frequency response, magnitude and phase errors of the proposed technique are smaller than those of [34]. In addition, due to no quantization error introduced, the frequency response, magnitude and phase errors of the proposed technique are both 0 at DC. When 


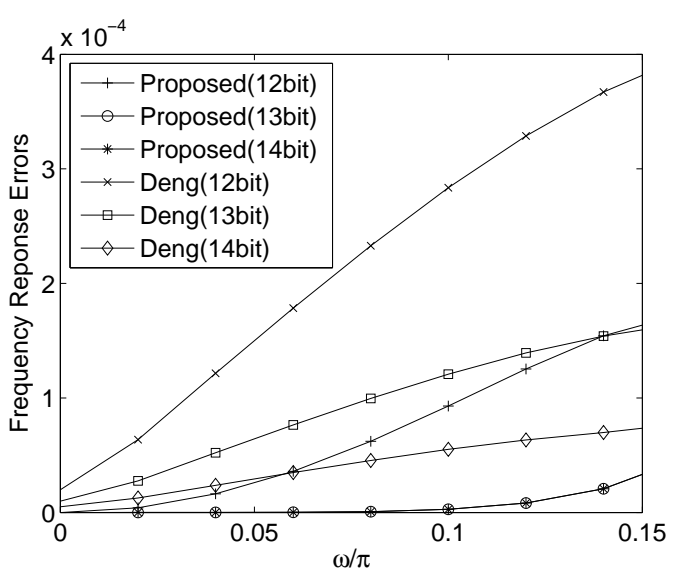

(a)

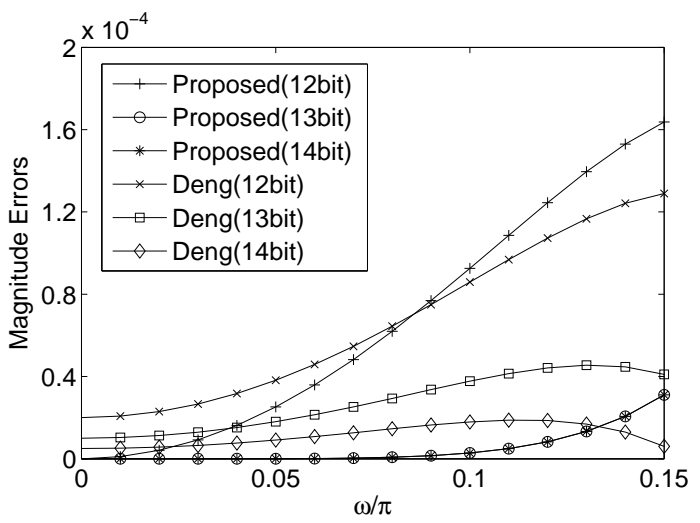

(b)

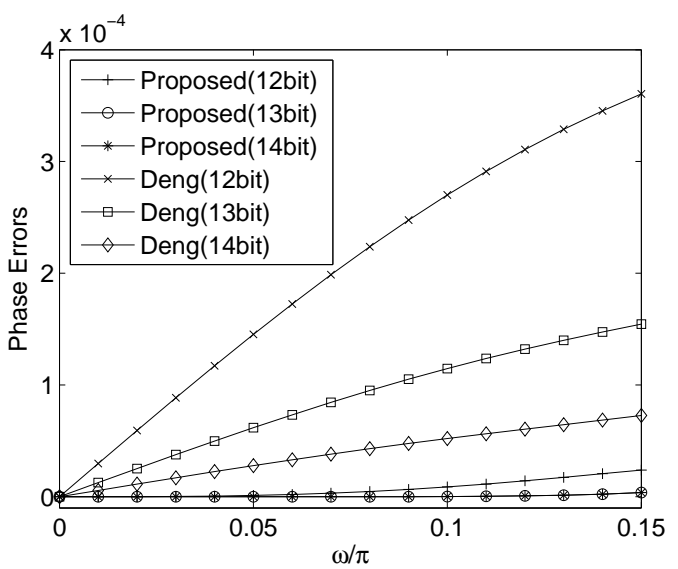

(c)

Figure 3.5. Example A: the (a) frequency response errors (b) magnitude errors and (c) phase errors of the 5th order Lagrange-type VFD filters implemented by the proposed technique and the technique in [34]. 
the coefficients are implemented with 12-bit word length, though rounding errors happen to the integer coefficient matrix, the resulting frequency response, magnitude and phase response both are smaller than that of [34] for the frequency close to DC.

\subsubsection{Design of a 6th order Lagrange-type VFD filter}

In this example, a 6th order Lagrange-type VFD FIR filter is designed using the proposed technique. In addition, the multiplierless technique [130] is applied in the synthesis of the integer coefficients to further reduce the computational complexity.

According to (3.16), the coefficient matrix of a 6th order Lagrange-type VFD FIR filter is calculated as,

$$
\widetilde{\mathbf{Q}}=\frac{1}{720}\left(\begin{array}{cccccccc}
0 & 0 & 0 & 720 & 0 & 0 & 0 \\
-12 & 108 & -540 & 0 & 540 & -108 & 12 \\
4 & -54 & 540 & -980 & 540 & -54 & 4 \\
15 & -120 & 195 & 0 & -195 & 120 & -15 \\
-5 & 60 & -195 & 280 & -195 & 60 & -5 \\
-3 & 12 & -15 & 0 & 15 & -12 & 3 \\
1 & -6 & 15 & -20 & 15 & -6 & 1
\end{array}\right)
$$

Scaled by a factor $c=720, c \widetilde{\mathbf{Q}}$ is an integer matrix. From (3.28), the 4 polynomials to be synthesized are:

$$
\begin{aligned}
& P_{0}(d)=4 d-5 d^{3}+d^{5}, \\
& P_{1}(d)=-54 d+60 d^{3}-6 d^{5}, \\
& P_{2}(d)=540 d-195 d^{3}+15 d^{5}, \\
& P_{3}(d)=-980 d+280 d^{3}-20 d^{5} .
\end{aligned}
$$

The overall polynomial structure is illustrated in Figure 3.6. 


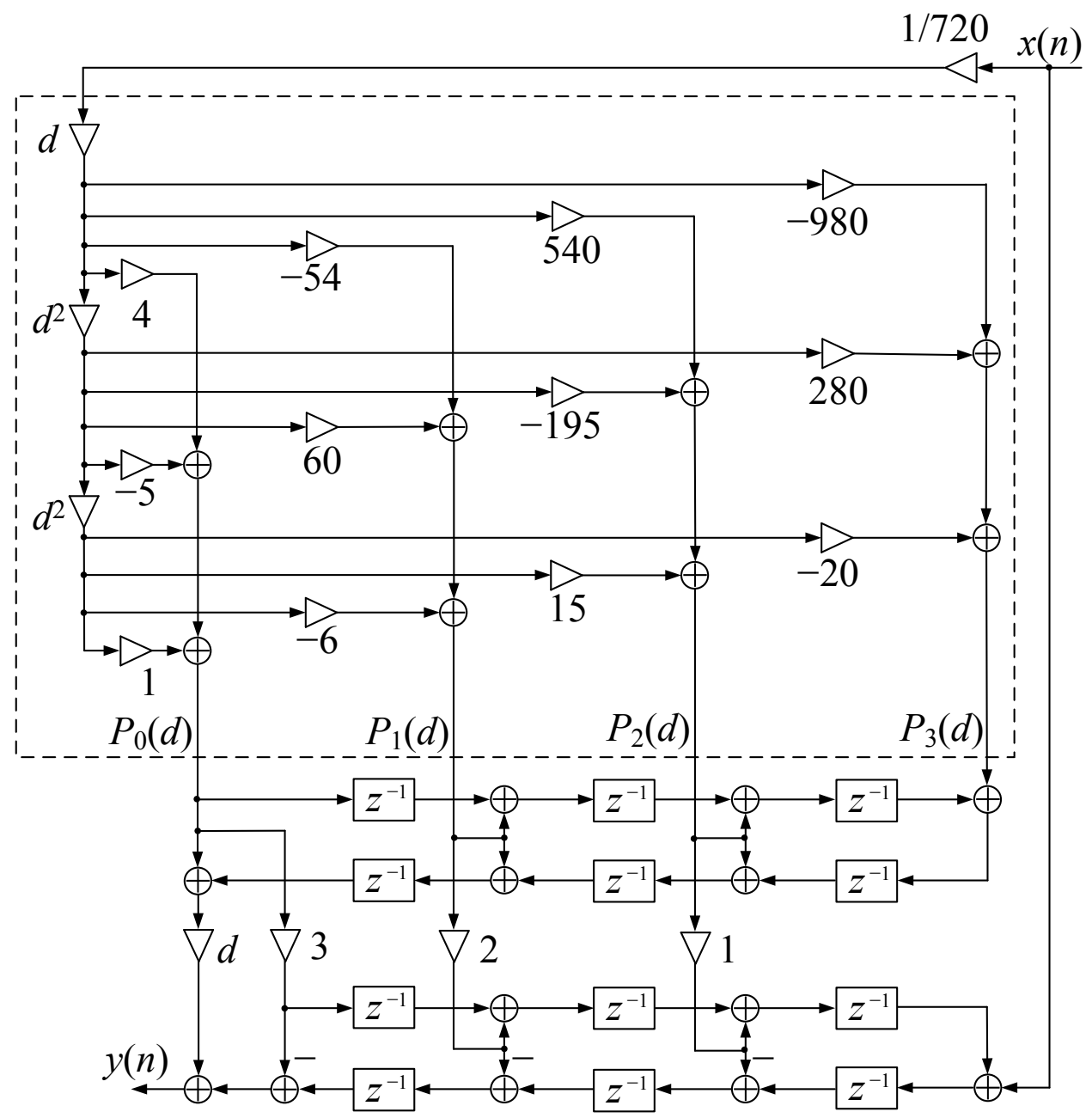

Figure 3.6. Polynomial structure for the 6th order $(N=6)$ Lagrange-type VFD filters.

In Figure 3.6, 20 multiplications in total are required for each output sample. The numbers of multiplications required in the direct implementation and the implementation exploiting subfilter symmetry in [34] are 45 and 27, respectively. Since all the coefficients are integer values, they can be synthesized by using an adder-shift network to further reduce the complexity. The BHM algorithm [130] for the synthesis of the matrix multiplication is applied. Using not less than 10 bits, the coefficients can be synthesized with full precisions. The synthesis of the coefficient multiplication (the block in the dash box in Figure 3.6) is shown in Figure 3.7. Twenty five adders are used in total where 12 adders are used to synthesize the 11 constant coefficient multipliers required in the 


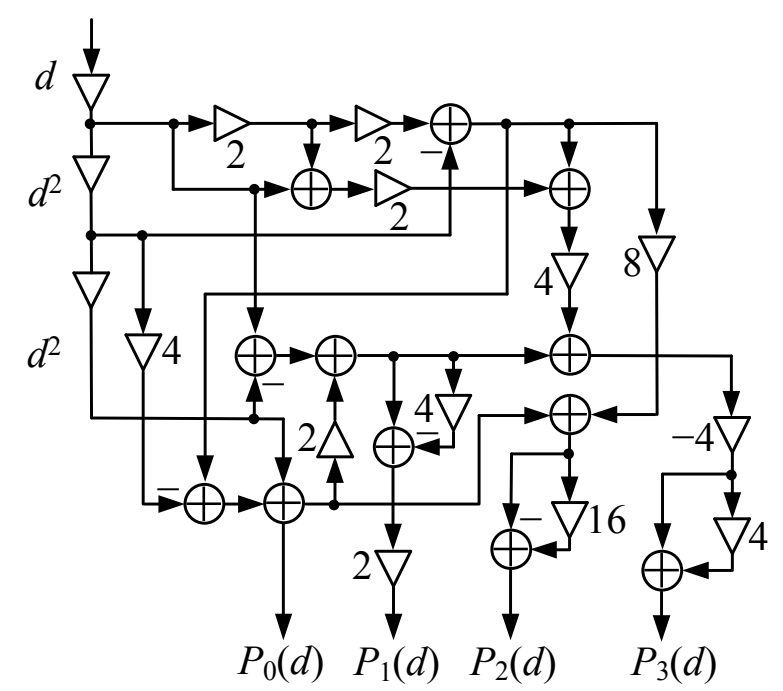

Figure 3.7. MCM techniques applied to the constant matrix multiplication.

Table 3.1

THE NUMBERS OF THE MULTIPLIER BLOCK ADDERS REQUIRED FOR THE SYNTHESIS OF THE COEFFICIENT MATRICES FROM THE PROPOSED TECHNIQUE AND THE TECHNIQUE IN [34] WITH 9-, 10- AND 11-BIT PRECISIONS, RESPECTIVELY.

\begin{tabular}{c|c|c|c}
\hline \hline & 9 bit & 10 bit & 11 bit \\
\hline Proposed & 11 & 12 & 12 \\
\hline The technique in [34] & 21 & 26 & 27 \\
\hline \hline
\end{tabular}

proposed structure and the remaining 13 adders are for the structure adders. The coefficient matrix in [34] containing 21 multipliers can be synthesized using the same BHM algorithm [130]. In comparison, Table 3.1 lists the number of multiplier block adders required for the synthesis of the coefficient matrices from the proposed technique and from [34] with 9-, 10- and 11-bit precisions, respectively.

From Table 3.1, it can be seen that the proposed technique achieves significantly efficient synthesis of the subfilter coefficients due to the dependance of the integer subfilter coefficients after scale.

The frequency response, magnitude and phase errors with fractional delay $d=0.4$ of the proposed technique and the technique in [34] with 9-, 10- and 11-bit precisions are 


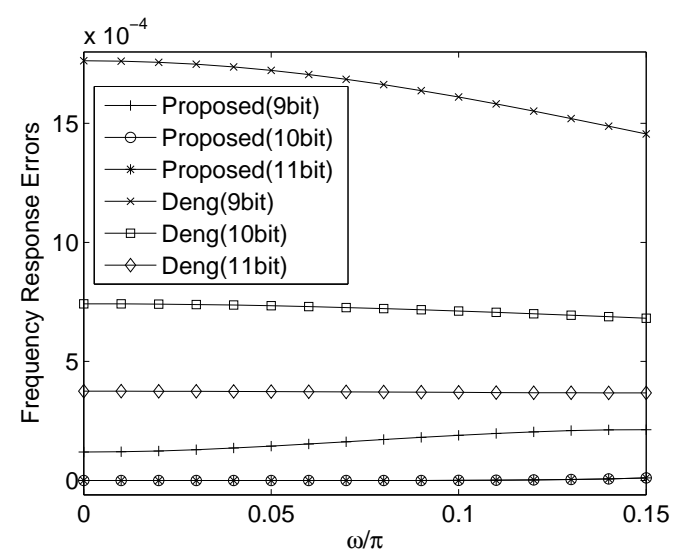

(a)

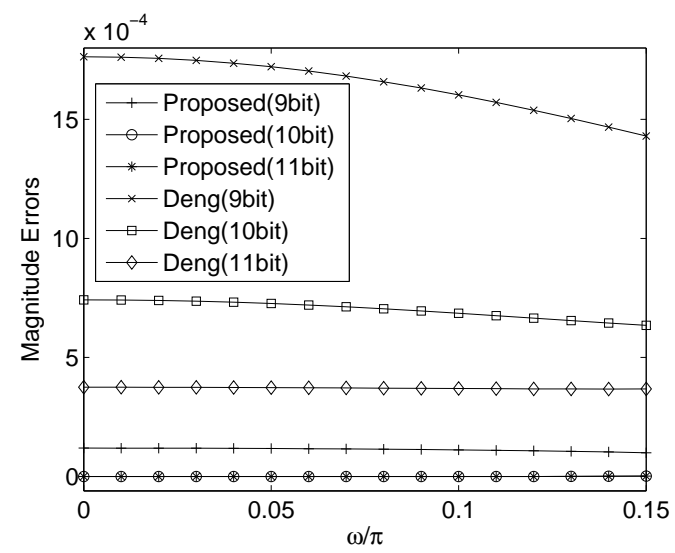

(b)

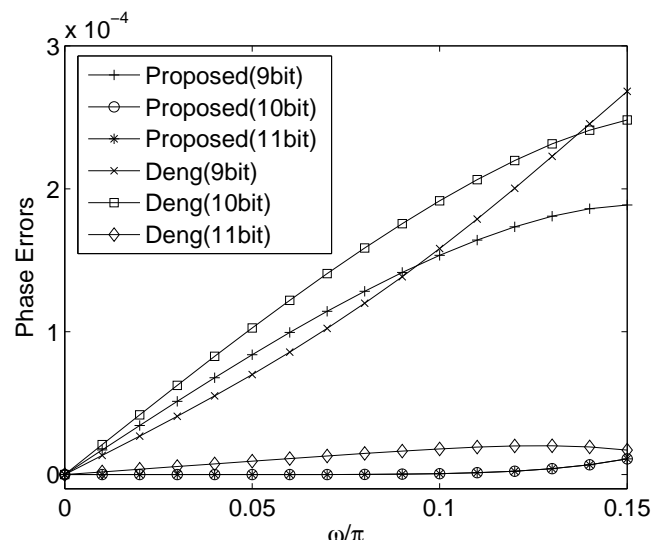

(c)

Figure 3.8. Example B: the (a) frequency response errors, (b) magnitude errors and (c) phase errors of the 6th order Lagrange-type VFD filters implemented by the proposed technique and the technique in [34]. 
shown in Figures 3.8(a), (b) and (c), respectively.

From Figures 3.8, it can be seen that the proposed technique also achieves significantly smaller frequency response errors than those of [34] when the subfilter coefficients are represent in 10 and 11 bits, respectively. Even rounding error occurs when the coefficients are represent in 9 bits, the frequency response of the proposed technique is still much better than that of [34].

It should be noted that the proposed structure can also be used in the implementation of the Farrow structure based truncated Lagrange-type VFD filters [33], which is an alternative for wide-band VFD filter design.

Although the proposed implementation structure has advantages on the low computational complexity and low rounding errors, it suffers from transients because the polynomials are realized before the filtering, and transient occurs when fractional delay changes. Hence, the proposed implementation structure is only applicable when the the fractional delay does not change often and transients are allowable. Similar to [58], the proposed structure provides an alternative implementation of VFD filters with low complexity at a price of transient issues.

\subsection{Summary}

This chapter investigates the subfilter coefficients of the Lagrange interpolation implemented in the Farrow structure and three properties of the subfilter coefficients are discovered. Making use of these properties, a new implementation structure is proposed.

The implementation complexity of the proposed structure is reduced nearly by half. The frequency response errors are also reduced when finite wordlength coefficients are implemented. It should be mentioned that the Lagrange interpolation method is attractive for VFD filter design due to its closed-form formula. However, the Lagrange interpolator can achieve low approximation errors only at low frequencies and the errors at high 
frequencies may be several orders higher than those at low frequencies. Therefore, it is not suitable for the design of wide band VFD filters. Though some techniques such as that in [33] have been proposed to solve this problem, the improved performance of the Lagrange interpolator is still not significant, compared with those obtained using optimization design techniques. 


\section{Chapter 4}

\section{Investigation on the Optimization Criteria for the Design of VFD Filters}

In addition to the closed-form designs of VFD filters such as the Lagrange interpolator described in Chapter 3, VFD filters are also numerically optimized in frequency domain to accurately meet specified requirements [45-71,78-87]. Conventionally, the error of the frequency response deviated from the ideal one is minimized in the optimization of the VFD filters [50-71,79-87, 89,90]. Besides the frequency response errors, phase errors $[80,81]$, phase delay errors $[52,54,79]$, group delay errors $[55,57,71]$, or some combinations of the above three errors $[53,59,85]$ are additionally considered in literature. Recently, more attentions are paid to minimize the group delay errors in addition to the frequency response errors $[57,71]$.

However, from Parseval's theorem, the error energy in time domain for the VFD filter used to estimate the instantaneous delayed-samples is equal to the error energy in frequency domain, i.e., the time domain error energy is determined by the signal energy and the energy of the frequency response errors only. Furthermore, as reviewed in Chapter 2, group delay physically measures the delay of magnitude envelope of a signal and phase measures the delay of instantaneous samples [131]. Therefore, a question raised is what the impact of the group delay errors is on the time domain estimation errors. While the group delay certainly is the measurement of envelop delays of signals, in this Chapter, the 
analysis upon the impact of the group delay error on the time domain error is performed when instantaneous samples are concerned.

\subsection{Review on Design Criteria of VFD Filters}

The ideal frequency response of a VFD filter, denoted as $\hat{H}\left(e^{j \omega}, d\right)$, is $e^{-j \omega d}$, where $d$ is a fraction varying in the range of $[-0.5,0.5]$. Let $H\left(e^{j \omega}, d\right)$ denote the frequency response

of the VFD filter to approximate the ideal response $\hat{H}\left(e^{j \omega}, d\right)$. As reviewed in Chapter 2 , the magnitude error, phase error and group delay error, denoted as $\delta_{m}(\omega, d), \delta_{\Phi}(\omega, d)$ and $\delta_{g}(\omega, d)$, respectively, are given as,

$$
\begin{gathered}
\delta_{m}(\omega, d)=\left|H\left(e^{j \omega}, d\right)\right|-1, \\
\delta_{\Phi}(\omega, d)=\Phi(\omega, d)-(-\omega d) .
\end{gathered}
$$

and

$$
\delta_{g}(\omega, d)=-\frac{\partial \delta_{\Phi}(\omega, d)}{\partial \omega}=-\frac{\partial \Phi(\omega, d)}{\partial \omega}-d .
$$

The frequency response error $\delta_{f}(\omega, d)$ is given by,

$$
\begin{aligned}
\delta_{f}(\omega, d) & =\left|H\left(e^{j \omega}, d\right)-\hat{H}\left(e^{j \omega}, d\right)\right| \\
& =\left|\left(1+\delta_{m}(\omega, d)\right) e^{-j\left(\omega d-\delta_{\Phi}(\omega, d)\right)}-e^{-j \omega d}\right| \\
& =\sqrt{\left(1+\delta_{m}(\omega, d)\right)^{2}+1-2\left(1+\delta_{m}(\omega, d)\right) \cos \left(\delta_{\Phi}(\omega, d)\right)}
\end{aligned}
$$

If $\delta_{\Phi}(\omega, d)$ and $\delta_{m}(\omega, d)$ are much smaller than 1, ignoring the third and high order error terms, (4.4) is simplified to

$$
\delta_{f}(\omega, d) \approx \sqrt{\delta_{m}^{2}(\omega, d)+\delta_{\Phi}^{2}(\omega, d)}
$$

It is worth mentioning that the ignored terms in (4.5) are the higher power terms of $\delta_{\Phi}$, i.e., $\delta_{\Phi}^{3}, \delta_{\Phi}^{4}$, ect., and have nothing to do with $\delta_{g}$, the derivative of $\delta_{\Phi}$.

In literature, besides the frequency responses given in (4.4), more attention is recently paid to minimize (4.3) and (4.4) simultaneously [57, 71]. 
In practical applications, the time-domain magnitude errors of the predicted instantaneous delayed samples with respect to the ideal delayed samples are the concern in the design of filters. In the following sections, the relations of the time-domain errors of the estimated samples with $\delta_{f}(\omega, d)$ and $\delta_{g}(\omega, d)$ are analyzed and simulated.

\subsection{Theoretical Analysis}

When a VFD filter is used to predict the delayed samples, say delayed by a fraction $d$, for input signal $x_{n}$, let $\hat{y}_{n}(d)$ denote the ideally delayed sample, and $y_{n}(d)$ the output signal. Through filtering, $y_{n}(d)$ is obtained as,

$$
y_{n}(d)=x_{n} * h_{n}(d)=\sum_{k=-\infty}^{\infty} h_{k}(d) x_{n-k}
$$

where $h_{n}(d)$ corresponds to the impulse responses of the filter generating the fractional delay $d$ and '*' represents the convolution operation. The error energy of the estimated samples with respect to the ideally delayed samples, denoted as $e_{t}^{2}(d)$, is given by

$$
e_{t}^{2}(d)=\sum_{n=-\infty}^{\infty}\left(h_{n}(d) * x_{n}-\hat{y}_{n}(d)\right)^{2} \text {. }
$$

When the signal $x_{n}$ has a continuous spectrum, the error function (4.7) can be written in frequency domain via Parseval theorem, i.e.,

$$
\begin{aligned}
e_{t}^{2}(d) & =\frac{1}{2 \pi} \int_{-\pi}^{\pi}\left|X\left(e^{j \omega}\right)\left(H\left(e^{j \omega}, d\right)-\hat{H}\left(e^{j \omega}, d\right)\right)\right|^{2} d \omega \\
& =\frac{1}{2 \pi} \int_{-\pi}^{\pi}\left|X\left(e^{j \omega}\right)\right|^{2} \delta_{f}^{2}(\omega, d) d \omega
\end{aligned}
$$

where $X\left(e^{j \omega}\right)$ is the discrete-time Fourier transform (DTFT) of $x_{n}$. When the input signal is bandlimited, the integral range is reduced accordingly. And if the signal contains only discrete frequency components, i.e., a sum of a limited number of sinusoid waves, the time domain error and frequency domain error can be rewritten as

$$
e_{t}^{2}(d)=\sum_{n=0}^{N-1}\left(h_{n}(d) * x_{n}-\hat{y}_{n}(d)\right)^{2}=\frac{1}{N} \sum_{k=0}^{N-1}|X(k)|^{2} \delta_{f}^{2}\left(\frac{2 k \pi}{N}, d\right)
$$


where $X(k)$ is the $N$-point discrete Fourier transform (DFT) of $x_{n}$.

From (4.8) and (4.9), it can be seen that other than the energy of the signal itself, the estimation error energy is determined by $\delta_{f}(\omega, d)$ or $\delta_{f}\left(\frac{2 k \pi}{N}, d\right)$.

Detailed to a particular time domain output sample $y_{n}(d)$, the analysis is given in the following.

Assume that signal $x_{n}$, for $n=0, \ldots, N$ is a sequence consisting of $M$ frequency component $\omega_{i}$, each with a magnitude $a_{i}$ and an initial phase $\psi_{i}$, for $i=0, \ldots, M-1$, given by,

$$
x_{n}=\sum_{i=0}^{M-1} a_{i} \sin \left(\omega_{i} n+\psi_{i}\right)
$$

When $x_{n}$ is filtered by a VFD filter with magnitude response $\left|H\left(e^{j \omega}, d\right)\right|$ and phase response $\Phi(\omega, d)$, the delayed samples are generated as,

$$
y_{n}(d)=\sum_{i=0}^{M-1} a_{i}\left|H\left(e^{j \omega_{i}}, d\right)\right| \sin \left[\omega_{i} n+\psi_{i}+\Phi\left(\omega_{i}, d\right)\right]
$$

and the ideally delayed sample $\hat{y}_{n}(d)$ is given by,

$$
\hat{y}_{n}(d)=\sum_{i=0}^{M-1} a_{i} \sin \left[\omega_{i}(n-d)+\psi_{i}\right]
$$

Thus, the estimated time domain error of the delayed sample, $y_{n}(d)$, with respect to the ideal delayed sample, $\hat{y}_{n}(d)$, denoted as $\epsilon(n, d)$, is given by

$$
\epsilon(n, d)=\left|y_{n}(d)-\hat{y}_{n}(d)\right|=\left|\sum_{i=0}^{M-1} a_{i} \epsilon_{i}(n, d)\right|
$$


where $\epsilon_{i}(n, d)$ is given as

$$
\begin{aligned}
\epsilon_{i}(n, d) & =\left(1+\delta_{m}\right) \sin \left[\omega_{i} n+\psi_{i}+\Phi\left(\omega_{i}, d\right)\right]-\sin \left[\omega_{i}(n-d)+\psi_{i}\right] \\
& =\left(1+\delta_{m}\right) \sin \left[\omega_{i}(n-d)+\psi_{i}+\delta_{\Phi}\right]-\sin \left[\omega_{i}(n-d)+\psi_{i}\right] \\
& =\delta_{m} \sin \left[\omega_{i}(n-d)+\psi_{i}+\delta_{\Phi}\right]+2 \sin \frac{\delta_{\Phi}}{2} \cos \left[\omega_{i}(n-d)+\psi_{i}+\frac{\delta_{\Phi}}{2}\right] \\
& =\delta_{m} \sin \left[\omega_{i}(n-d)+\psi_{i}+\delta_{\Phi}\right]+2 \sin \frac{\delta_{\Phi}}{2} \cos \left[\omega_{i}(n-d)+\psi_{i}+\delta_{\Phi}-\frac{\delta_{\Phi}}{2}\right] \\
& =\delta_{m} \sin \left[\omega_{i}(n-d)+\psi_{i}+\delta_{\Phi}\right] \\
& +2 \sin \frac{\delta_{\Phi}}{2}\left(\cos \left[\omega_{i}(n-d)+\psi_{i}+\delta_{\Phi}\right] \cos \frac{\delta_{\Phi}}{2}+\sin \left[\omega_{i}(n-d)+\psi_{i}+\delta_{\Phi}\right] \sin \frac{\delta_{\Phi}}{2}\right) \\
& =\sin \delta_{\Phi} \cos \left[\omega_{i}(n-d)+\psi_{i}+\delta_{\Phi}\right]+\left(\delta_{m}+2 \sin ^{2} \frac{\delta_{\Phi}}{2}\right) \sin \left[\omega_{i}(n-d)+\psi_{i}+\delta_{\Phi}\right] \\
& =A_{i} \sin \left[\omega_{i}(n-d)+\psi_{i}+\delta_{\Phi}+\alpha_{i}\right],
\end{aligned}
$$

where $A_{i}$ and $\alpha_{i}$ are given by

$$
A_{i}=\sqrt{\left(\delta_{m}+2 \sin ^{2} \frac{\delta_{\Phi}}{2}\right)^{2}+\sin ^{2} \delta_{\Phi}}=\sqrt{\left(1+\delta_{m}\right)^{2}+1-2\left(1+\delta_{m}\right) \cos \delta_{\Phi}}
$$

and

$$
\alpha_{i}= \begin{cases}\arcsin \left(\frac{\sin \delta_{\Phi}}{A_{i}}\right) & \text { if } \delta_{m}+2 \sin ^{2} \frac{\delta_{\Phi}}{2} \geq 0 \\ \pi-\arcsin \left(\frac{\sin \delta_{\Phi}}{A_{i}}\right) & \text { otherwise. }\end{cases}
$$

In (4.13-4.16), $\delta_{m}$ and $\delta_{\Phi}$ are short for $\delta_{m}\left(\omega_{i}, d\right)$ and $\delta_{\Phi}\left(\omega_{i}, d\right)$, respectively. Since (4.15) is the same as (4.4), approximating (4.15) in the same way as (4.4) when $\delta_{m}(\omega, d)$ and $\delta_{\Phi}(\omega, d)$ are small, (4.13) can be simplified as

$$
\epsilon(n, d) \leq \sum_{i=0}^{M-1}\left|a_{i} A_{i}\right| \approx \sum_{i=0}^{M-1} a_{i} \sqrt{\delta_{m}^{2}\left(\omega_{i}, d\right)+\delta_{\Phi}^{2}\left(\omega_{i}, d\right)}
$$

Equation (4.17) shows when $\delta_{f}(\omega, d)$ in (4.4) is minimized, the upper bound of $\epsilon(n, d)$ in (4.13) is also minimized.

The above analysis shows that the time domain estimation error (for instantaneous delayed samples) are determined by the frequency response error $\delta_{f}(\omega, d)$, which is further determined by the magnitude error $\delta_{m}(\omega, d)$ and phase error $\delta_{\Phi}(\omega, d)$. 
For the group delay error $\delta_{g}(\omega, d)$, it relates with $\delta_{\Phi}(\omega, d)$ by $(4.3)$, so that for the same phase error energy or maximum phase error, the group delay error can be any value depending on the smoothness of $\delta_{\Phi}(\omega, d)$ with respect to the frequency. On the other hand, when a sample requires a fractional delay $d$, it corresponds to a phase shift amount $\omega d$. However, the samples with phase shift of $\omega d+\Phi_{0}$ for any $\Phi_{0}$ all satisfy the condition that the group delay is $d$ (due to the derivative relation), but these samples are not the samples with sample delay $d$.

Therefore, when VFD filters are optimized by minimizing $\delta_{f}(\omega, d)$, either in least square sense or in minimax sense, the corresponding time domain error is also minimized. In the case that $\delta_{g}(\omega, d)$ is additionally minimized, if the minimization of $\delta_{g}(\omega, d)$ does not affect $\delta_{f}(\omega, d)$, the time domain error is not affected as well; if the minimization of $\delta_{g}(\omega, d)$ is at the price of increasing $\delta_{f}(\omega, d)$, the time domain error is increased as well. On the other hand, it is impossible for a converged optimization algorithm that $\delta_{f}(\omega, d)$ is further improved when additional objective $\delta_{g}(\omega, d)$ is minimized or even just constrained.

The above analysis result is not surprising since group delay measures the delay of the magnitude envelop of a signal, not the delay of the instantaneous samples [131]. Phase measures the later.

Overall, Parseval's theorem in (4.8) specifies the time domain error in least squares sense, while (4.17) gives the maximum error bound of an instantaneous sample. The bound in (4.17) is conservative though, because the effect of the phase error and magnitude error in the time domain may cancel each other, and the errors of different frequency components may also cancel each other. 
Table 4.1

FREQUENCY RESPONSE ERROR $\delta_{f}$, MAGNITUDE ERROR $\delta_{m}$, PHASE ERROR $\delta_{\Phi}$ AND GROUP DELAY ERROR $\delta_{g}$ AT $\omega_{1}$ OF $F A$ AND $F B$, AND THE CORRESPONDING MAXIMUM SAMPLE ESTIMATION ERROR $\epsilon_{m}$ AND THE TOTAL TIME DOMAIN ERROR ENERGY $e_{t}^{2}$ FOR SINGLE FREQUENCY SINUSOID INPUT SIGNAL.

\begin{tabular}{c|c|c|c|c|c|c|c|c|c|c|c|c|c}
\hline \hline \multirow{2}{*}{$d$} & \multicolumn{7}{|c|}{ Frequency domain error } & \multicolumn{4}{c}{ Time domain error } \\
\cline { 2 - 14 } & $\delta_{f} \times 10^{5}$ & $\delta_{m} \times 10^{5}$ & $\delta_{\Phi} \times 10^{5}$ & \multicolumn{2}{|c|}{$\delta_{g} \times 10^{5}$} & \multicolumn{3}{|c|}{$\epsilon_{m} \times 10^{5}$} & \multicolumn{2}{|c|}{$e_{t}^{2} \times 10^{6}$} \\
\cline { 2 - 14 } & $F A$ & $F B$ & $F A$ & $F B$ & $F A$ & $F B$ & $F A$ & $F B$ & $F A$ & $F B$ & $F A$ & $F B$ \\
\hline \pm 0.1 & 7.49 & 6.14 & 6.84 & 1.17 & 3.04 & 6.02 & 936 & 53.6 & 7.49 & 6.14 & 2.80 & 1.88 \\
\hline \pm 0.2 & 7.38 & 11.5 & 4.16 & 4.32 & 6.09 & 10.6 & 1870 & 97.2 & 7.38 & 11.5 & 2.72 & 6.57 \\
\hline \pm 0.3 & 9.17 & 15.8 & 0.31 & 8.64 & 9.16 & 13.2 & 2801 & 121 & 9.17 & 15.8 & 4.20 & 12.4 \\
\hline \pm 0.4 & 13.9 & 18.8 & 6.55 & 13.1 & 12.3 & 13.4 & 3728 & 122 & 13.9 & 18.8 & 9.66 & 17.6 \\
\hline \pm 0.5 & 21.2 & 17.4 & 14.6 & 14.4 & 15.4 & 9.77 & 4650 & 107 & 21.2 & 17.4 & 22.5 & 15.2 \\
\hline
\end{tabular}

\subsection{Verification by Numerical Examples}

Two pairs of VFD filters, denoted as Pair 1 and Pair 2, are designed to perform VFD in the frequency range $[0,0.8 \pi]$. In Pair 1, two FIR VFD filters, denoted as $F A$ and $F B$ are designed using the techniques in [93] and [64], respectively. In Pair 2, two allpass VFD filters, denoted as $F C$ and $F D$, are designed using the technique in [69]. Since allpass filters have an unit magnitude response in the full frequency range, the simulations to the allpass filters are the supplements to that of FIR filters to show the time domain errors caused by only phase errors and group delay errors.

To show the contrast, $F A$ and $F C$ are designed purposely with large $\delta_{g}$, compared with $F B$ and $F D$, respectively. Three types of signals, i.e., single frequency sinusoid signal, white noise signal, and deterministic wide band chirp signal, are tested. The related frequency domain errors of the filters are listed along with each test.

\subsubsection{Single Frequency Sinusoid Signal}

Two sequences of sinusoid signals, each containing a single frequency with unit magnitudes and random initial phases, are generated with $N=10^{4}$ samples. The frequencies 
Table 4.2

FREQUENCY RESPONSE ERROR $\delta_{f}$, MAGNITUDE ERROR $\delta_{m}$, PHASE ERROR $\delta_{\Phi}$ AND GROUP DELAY ERROR $\delta_{g}$ AT $\omega_{2}$ OF $F C$ AND $F D$, AND THE CORRESPONDING MAXIMUM SAMPLE ESTIMATION ERROR $\epsilon_{m}$ AND THE TOTAL TIME DOMAIN ERROR ENERGY $e_{t}^{2}$ FOR SINGLE FREQUENCY SINUSOID INPUT SIGNAL.

\begin{tabular}{c|c|c|c|c|c|c|c|c|c|c}
\hline \hline \multirow{2}{*}{$d$} & \multicolumn{4}{|c|}{ Frequency domain error } & \multicolumn{3}{c}{ Time domain error } \\
\cline { 2 - 11 } & $\delta_{f} \times 10^{5}$ & $\delta_{\Phi} \times 10^{5}$ & $\delta_{g} \times 10^{3}$ & \multicolumn{2}{c}{$\epsilon_{m} \times 10^{5}$} & \multicolumn{2}{|c}{$e_{t}^{2} \times 10^{6}$} \\
\cline { 2 - 12 } & $F C$ & $F D$ & $F C$ & $F D$ & $F C$ & $F D$ & $F C$ & $F D$ & $F C$ & $F D$ \\
\hline 0.1 & 10.11 & 0.169 & 10.11 & 0.169 & 36.88 & 9.221 & 10.11 & 0.169 & 5.108 & 0.001 \\
\hline-0.1 & 6.380 & 3.088 & 6.380 & 3.088 & 12.68 & 4.499 & 6.380 & 3.088 & 2.305 & 0.477 \\
\hline 0.2 & 18.53 & 9.427 & 18.53 & 9.427 & 122.5 & 25.83 & 18.53 & 9.427 & 17.17 & 4.444 \\
\hline-0.2 & 8.895 & 6.384 & 8.895 & 6.384 & 14.41 & 6.118 & 8.894 & 6.384 & 3.956 & 2.038 \\
\hline 0.3 & 3.910 & 37.86 & 3.910 & 37.86 & 299.8 & 53.59 & 3.910 & 37.86 & 0.764 & 71.68 \\
\hline-0.3 & 8.784 & 8.559 & 8.784 & 8.559 & 11.98 & 6.097 & 8.784 & 8.559 & 3.858 & 3.662 \\
\hline 0.4 & 100.2 & 101.8 & 100.2 & 101.8 & 640.7 & 97.82 & 100.2 & 101.8 & 501.9 & 518.0 \\
\hline-0.4 & 7.318 & 9.279 & 7.318 & 9.279 & 8.590 & 5.251 & 7.317 & 9.279 & 2.677 & 4.305 \\
\hline 0.5 & 483.2 & 226.3 & 483.2 & 226.3 & 1257 & 165.9 & 483.2 & 226.2 & 11674 & 2559 \\
\hline-0.5 & 5.412 & 8.723 & 5.412 & 8.723 & 5.556 & 4.087 & 5.412 & 8.723 & 1.464 & 3.805 \\
\hline
\end{tabular}

of the two sinusoid sequences are arbitrarily selected as $\omega_{1}=0.630 \pi$ (for Pair 1) and $\omega_{2}=0.252 \pi$ (for Pair 2), respectively, for Test 1 using the filters in Pair 1 and Test 2 using the filters in Pair 2.

The sequences are processed by the two filters in each pair, respectively, with $d$ varying among the values of $\pm 0.1, \pm 0.2, \pm 0.3, \pm 0.4, \pm 0.5$, where for each $d$, in total 1,000 samples are processed by each filter. The resultant output samples are compared with the ideal delayed ones; the later is obtained according to (4.12). The frequency domain errors at $\omega_{1}$ for the filters $F A$ and $F B$ in Pair 1 are listed in Table 4.1 and that at $\omega_{2}$ for the filters $F C$ and FD in Pair 2 are listed in Table 4.2.

The time domain estimation errors introduced in Test 1 and Test 2 for different fractional delays, including the maximum sample error $\epsilon_{m}$ and total error energy $e_{t}^{2}$, are also listed in Tables 4.1 and 4.2, respectively. 


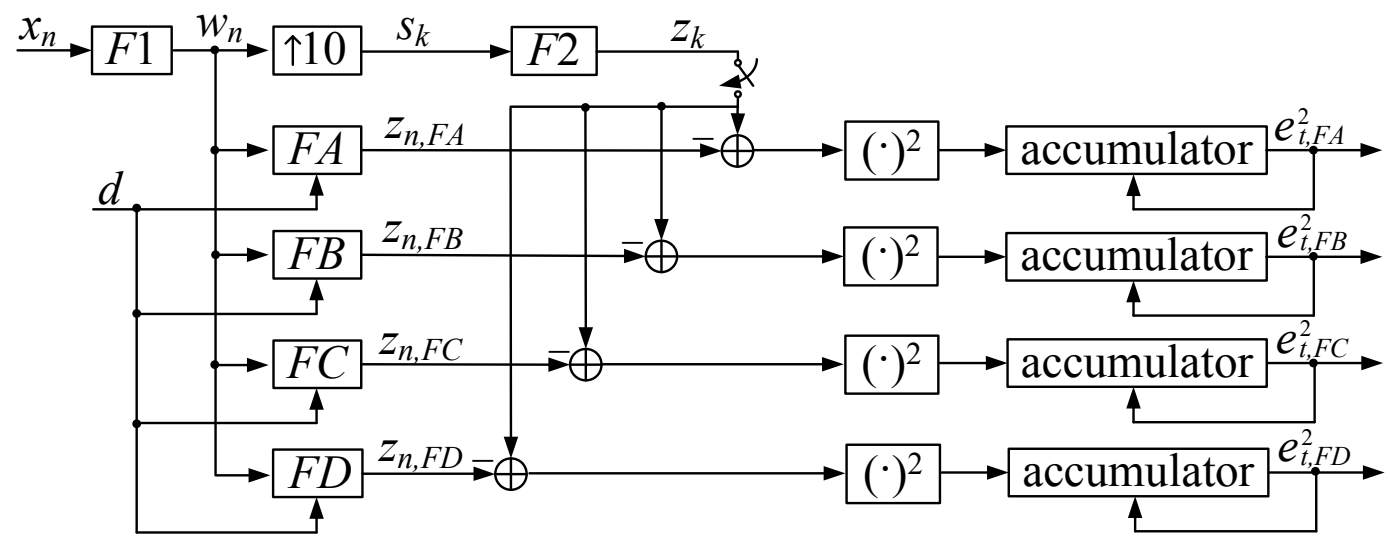

Figure 4.1. The time domain approximation system, where $(.)^{2}$ denotes the square operation.

From Tables 4.1 and 4.2 , it can be seen that $\delta_{f}$ follows the relation in (4.5) with $\delta_{m}$ and $\delta_{\Phi}$. Furthermore, the maximum time domain error $\epsilon_{m}$ is exactly the same as $\delta_{f}$; this is because that the single frequency sinusoid input is a special case of the analysis in (4.10)-(4.17), i.e., no cancelation among different frequency component exists. The bound given in (4.17) becomes the actual maximum error, because the sine term in (4.13) always has chances to reach 1 .

For the same reason, the obtained simulation result $e_{t}^{2}$ is also exactly the same as that obtained theoretically by (4.9). This example verifies that $\delta_{f}$ determines the time domain error; the significantly smaller $\delta_{g}$ in $F B$ and $F D$ have not slight influence to the time domain error, in Test 1 and Test 2, respectively.

\subsubsection{White Noise Signal}

The experiment is designed as follows: the Gaussian white noise sequence $x_{n}$ for $n=$ $0,1, \ldots, N$ is generated, with $N=10^{4}$. The spectrum is uniformly distributed in the normalized frequency range $[0, \pi]$. Since the VFD filters work in the frequency range $[0,0.8 \pi]$, a lowpass filter, denoted as $F 1$, with passband and stopband edges of $0.78 \pi$ and $0.80 \pi$, respectively, is applied to remove the signal frequency beyond $0.8 \pi$, as shown in Figure 4.1. The stopband attenuation of $F 1$ is as high as $150 \mathrm{~dB}$ to remove the unnecessary frequency components. The output sequence of $F 1$, denoted as $w_{n}$, is the input 
Table 4.3

$e_{f}^{2}$ AND $e_{g}^{2}$ GIVEN IN (4.18), OF $F A$ AND $F B$ FOR, AND THE CORRESPONDING TIME DOMAIN ERRORS $\epsilon_{m}$ AND $e_{t}^{2}$ FOR WHITE NOISE SIGNAL.

\begin{tabular}{c|c|c|c|c|c|c|c|c}
\hline \hline \multirow{2}{*}{$d$} & \multicolumn{3}{|c|}{ Frequency domain error } & \multicolumn{4}{c}{ Time domain error } \\
\cline { 2 - 9 } & \multicolumn{2}{|c|}{$e_{f}^{2} \times 10^{8}$} & \multicolumn{2}{|c}{$e_{g}^{2} \times 10^{4}$} & \multicolumn{3}{c}{$\epsilon_{m} \times 10^{4}$} & \multicolumn{2}{|c}{$e_{t}^{2} \times 10^{5}$} \\
\cline { 2 - 9 } & $F A$ & $F B$ & $F A$ & $F B$ & $F A$ & $F B$ & $F A$ & $F B$ \\
\hline 0.1 & 6.99 & 1.46 & 1.58 & 0.03 & 3.88 & 1.54 & 1.34 & 0.26 \\
-0.1 & & & & & 4.43 & 1.73 & 1.37 & 0.30 \\
\hline 0.2 & \multirow{2}{*}{4.32} & 5.38 & 6.33 & \multirow{2}{*}{0.11} & 2.86 & 3.32 & 0.80 & 0.96 \\
-0.2 & & & & & 2.99 & 4.11 & 0.89 & 1.11 \\
\hline 0.3 & \multirow{2}{*}{4.27} & \multirow{2}{*}{10.3} & \multirow{2}{*}{14.2} & \multirow{2}{*}{0.17} & 2.81 & 4.70 & 0.85 & 2.14 \\
-0.3 & & & & & 2.98 & 5.17 & 0.88 & 2.04 \\
\hline 0.4 & \multirow{2}{*}{12.1} & \multirow{2}{*}{14.2} & 25.3 & \multirow{2}{*}{0.19} & 5.01 & 6.27 & 2.32 & 2.79 \\
-0.4 & & & & & 5.10 & 5.19 & 2.35 & 2.59 \\
\hline 0.5 & \multirow{2}{*}{36.1} & \multirow{2}{*}{16.5} & 40.9 & \multirow{2}{*}{0.17} & 8.72 & 5.45 & 6.95 & 3.00 \\
-0.5 & & & & & 9.15 & 6.58 & 7.77 & 3.53 \\
\hline
\end{tabular}

sequence to the VFD filters.

The signal $w_{n}$ is then interpolated by a factor of 10 to obtain $s_{k}$, i.e.,

$$
s_{k}= \begin{cases}w_{n} & \text { if } k=10 n \text { for } n=0,1, \ldots, N, \\ 0 & \text { otherwise }\end{cases}
$$

The sequence $s_{k}$, for $k=0, \ldots, 10 N$, is thereafter passed through an anti-image filter, denoted as $F 2$, resulting in a sequence $z_{k}$ for $k=0,1, \ldots, 10 N . z_{k}$ contains delayedsamples with $d=-0.5,-0.4, \ldots, 0.4,0.5$. The filter $F 2$, with passband and stopband edges at $0.08 \pi$ and $0.12 \pi$, respectively, has ripples in the passband and stopband as small as $-170 \mathrm{~dB}$ to interpolate the signal with high fidelity.

The sequence $w_{n}$ is processed by the filters $F A, F B, F C$ and $F D$ with $d$ varying among the values of $\pm 0.1, \pm 0.2, \pm 0.3, \pm 0.4, \pm 0.5$. To maximally eliminate the effect of different error energy in frequency domain for different $d$, the appearing frequencies of different $d$ are the same, i.e., each $d$ among the 10 values appears 1,000 times. The 
Table 4.4

$e_{f}^{2}$ AND $e_{g}^{2}$ GIVEN IN (4.18), OF $F C$ AND $F D$ FOR, AND THE CORRESPONDING TIME DOMAIN ERRORS $\epsilon_{m}$ AND $e_{t}^{2}$ FOR WHITE NOISE SIGNAL.

\begin{tabular}{c|c|c|c|c|c|c|c|c}
\hline \hline \multirow{2}{*}{$d$} & \multicolumn{3}{|c|}{ Frequency domain error } & \multicolumn{3}{c}{ Time domain error } \\
\cline { 2 - 10 } & $e_{f}^{2} \times 10^{5}$ & $e_{g}^{2} \times 10^{3}$ & \multicolumn{2}{c}{$\epsilon_{m} \times 10^{3}$} & \multicolumn{2}{c}{$e_{t}^{2} \times 10^{3}$} \\
\cline { 2 - 10 } & $F C$ & $F D$ & $F C$ & $F D$ & $F C$ & $F D$ & $F C$ & $F D$ \\
\hline 0.1 & 0.529 & 0.715 & 6.678 & 0.716 & 2.636 & 3.343 & 0.750 & 1.033 \\
\hline-0.1 & 0.078 & 0.218 & 1.006 & 0.224 & 1.313 & 1.900 & 0.118 & 0.383 \\
\hline 0.2 & 5.271 & 4.996 & 66.24 & 4.926 & 10.38 & 9.981 & 8.136 & 7.577 \\
\hline-0.2 & 0.115 & 0.460 & 1.490 & 0.479 & 1.254 & 2.670 & 0.132 & 0.540 \\
\hline 0.3 & 28.86 & 19.29 & 361.6 & 18.68 & 22.94 & 17.34 & 41.20 & 29.22 \\
\hline-0.3 & 0.090 & 0.524 & 1.191 & 0.551 & 1.131 & 2.745 & 0.130 & 0.678 \\
\hline 0.4 & 122.3 & 57.94 & 1526 & 55.01 & 44.61 & 28.14 & 185.8 & 93.26 \\
\hline-0.4 & 0.053 & 0.448 & 0.714 & 0.474 & 0.695 & 2.133 & 0.070 & 0.555 \\
\hline 0.5 & 449.1 & 151.0 & 5526 & 140.4 & 94.22 & 51.89 & 716.1 & 224.9 \\
\hline-0.5 & 0.026 & 0.314 & 0.351 & 0.334 & 0.708 & 2.162 & 0.034 & 3.92 \\
\hline
\end{tabular}

resultant signals $z_{n, F A}, z_{n, F B}, z_{n, F C}$ and $z_{n, F D}$ are compared with the particular $z_{k}$ corresponding to every $d$ to obtain $\epsilon_{m, F A}, e_{t, F A}^{2}, \epsilon_{m, F B}, e_{t, F B}^{2}, \epsilon_{m, F C}, e_{t, F C}^{2}$ and $\epsilon_{m, F D}, e_{t, F D}^{2}$.

The frequency and time domain errors for $F A$ and $F B$ are listed in Table 4.3, where $e_{f}^{2}(d)$ and $e_{g}^{2}(d)$ are the energy of the frequency response error and group delay error, given by

$$
e_{f}^{2}(d)=2 \int_{a}^{b} \delta_{f}^{2}(\omega, d) d \omega \text { and } e_{g}^{2}(d)=2 \int_{a}^{b} \delta_{g}^{2}(\omega, d) d \omega
$$

respectively. In (4.18), $a=0$ and $b=0.8 \pi$. In the white noise input example, only the integrated errors for different $d$ in frequency domain are considered because the filter designed is not able to be equiripple over the entire frequency range. The time domain estimation errors for each particular $d$ are listed in Table 4.3 for the test using $F A$ and $F B$, and Table 4.4 for $F C$ and $F D$.

Tables 4.3 and 4.4 show that the time domain errors for each particular $d$ are consistent with the frequency response errors, i.e., larger $e_{f}^{2}$ results in larger $\epsilon_{m}$ and $e_{t}^{2}$ regardless 
of the values of $e_{g}^{2}$. In other words, the significantly smaller group delay error energy $e_{g}^{2}$ of $F B$ and $F D$ cannot improve the time domain performance. More specifically, the values of $e_{t}^{2}(d)$ computed by (4.18) from Tables 4.3 and 4.4 are very close to $\frac{e_{f}^{2}(d)}{2 \pi N}$. The slight differences between them are because although the spectrum of the white noise signal is flat, it is not as flat as a straight line.

For reference, the time domain differences between the sequence $y_{n}$ and $z_{10 n}$ are also calculated. The corresponding maximum error $\epsilon_{m 0}$ and error energy $e_{t 0}^{2}$ are $6.58 \times$ $10^{-9}$ and $2.50 \times 10^{-15}$, respectively. These values show that the interpolation system in Figure 4.1 controls the noise of individual sample to a low level, which is insignificant in the evaluation of $\epsilon_{m}$ and $e_{t}^{2}$.

\subsubsection{Chirp Signal}

Chirp signals are tested to show that the relation between the frequency domain and time domain holds for deterministic wide band signals as well. The chirp signal under test is given by,

$$
x_{n}=\cos \left(\omega_{n} n\right)
$$

where $\omega_{n}=\frac{n \pi}{N}$ for $N=2 \times 10^{4}$ and $n=0, \ldots, N$.

From (4.19), it can be seen that the frequency components carried by the signal at different time durations are different. More specifically, when $n$ is small, the energy is concentrated at low frequency, and when $n$ becomes larger, the signal energy concentrates on higher frequency. For example, in the sequence $x_{n}$ for $n=0, \ldots, 20,000$, the frequency spectrums of the signal segments for $n=0, \ldots, 999, n=1000, \ldots, 1999$, $n=2000, \ldots, 2999$ and $n=3000, \ldots, 3999$ are shown in Figures 4.2, respectively.

The simulation is conducted in the same way as that of white noise and the parameter $d$ is randomly varying among the values $\pm 0.1, \ldots, \pm 0.5$, where the duration of the signal segment for each delay value is also randomly selected from 1, 000 to 1,500. The actual order of the random selection of $d$ is given in Tables 4.5 and 4.6. 


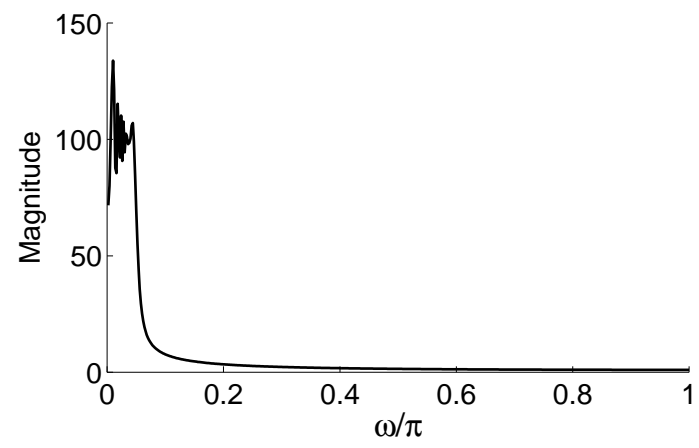

(a)

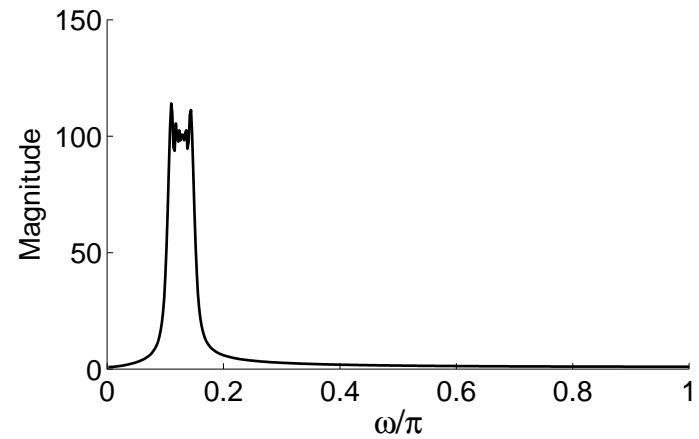

(c)

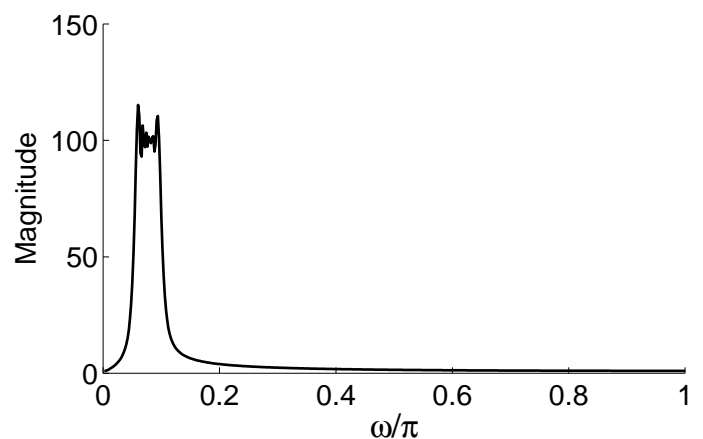

(b)

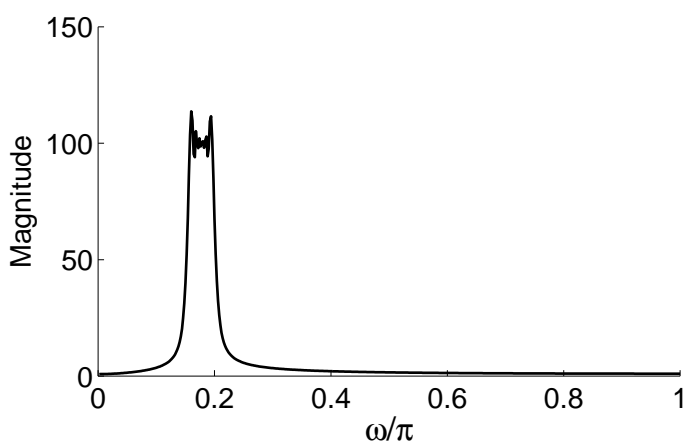

(d)

Figure 4.2. Frequency spectrum of signal segments of a chirp signal $x_{n}$ given in (4.19) for (a) $n=0, \ldots, 999$, (b) $n=1000, \ldots, 1999$, (c) $n=2000, \ldots, 2999$ and (d) $n=3000, \ldots, 3999$.

First, $e_{f}^{2}(d)$ and $e_{g}^{2}(d)$ which were used in the simulation for the white noise are used for chirp signal, and the integration range in (4.18) is the frequency spectrum range of the signal segment for each particular $d$. The frequency spectrum range of each signal segment is specified to the range that the magnitude of the spectrum is decreased to a certain percentage of the peak values. These error values are listed in Tables 4.5 and 4.6 for each selected $d$ of Pair 1 and Pair 2, respectively.

It can be seen that in most cases, larger $e_{f}^{2}$ causes larger $\epsilon_{m}$ and $e_{t}^{2}$, although the ratio of $e_{f, F A}^{2} / e_{f, F B}^{2}$ is no longer so consistent to $e_{t, F A}^{2} / e_{t, F B}^{2}$ as that in white noise simulation. 
Table 4.5

$e_{f}^{2}, e_{g}^{2}$ GIVEN IN (4.18), $\hat{e}_{f}^{2}, \hat{e}_{g}^{2}$ GIVEN IN (4.20) AND (4.21), OF $F A$ AND $F B$, AND THE TIME DOMAIN ERRORS $\epsilon_{m}$ AND $e_{t}^{2}$ FOR CHIRP INPUT SIGNAL.

\begin{tabular}{|c|c|c|c|c|c|c|c|c|c|c|c|c|}
\hline \multirow{3}{*}{$d$} & \multicolumn{8}{|c|}{ Frequency Domain Error } & \multicolumn{4}{|c|}{ Time Domain Error } \\
\hline & \multicolumn{2}{|c|}{$e_{f}^{2}(d) \times 10^{10}$} & \multicolumn{2}{|c|}{$e_{g}^{2}(d) \times 10^{8}$} & \multicolumn{2}{|c|}{$\hat{e}_{f}^{2}(d) \times 10^{5}$} & \multicolumn{2}{|c|}{$\hat{e}_{g}^{2}(d) \times 10^{3}$} & \multicolumn{2}{|c|}{$\epsilon_{m} \times 10^{8}$} & \multicolumn{2}{|c|}{$e_{t}^{2} \times 10^{5}$} \\
\hline & $F A$ & $F B$ & $F A$ & $F B$ & $F A$ & $F B$ & $F A$ & $F B$ & $F A$ & $F B$ & $F A$ & $F B$ \\
\hline 0.1 & 14.55 & 2.52 & 318.87 & 3.58 & 0.93 & 0.22 & 19.60 & 0.12 & 1.79 & 0.78 & 0.93 & 0.22 \\
\hline-0.3 & 9.52 & 19.29 & 09.86 & 25.62 & 53 & 0.52 & 175.57 & 2.34 & 1.60 & 1.67 & 0.51 & 0.52 \\
\hline 0.2 & 9.70 & 9.54 & 41.76 & 16.41 & 0.56 & 0.81 & 80.36 & 0.55 & 1.43 & 1.51 & 0.57 & 0.81 \\
\hline 0.4 & 26.37 & 40.33 & 5529.69 & 44.66 & 1.48 & 1.93 & 320.75 & 3.20 & 2.53 & 3.09 & 1.51 & 1.94 \\
\hline 0.5 & 2.60 & .27 & 684.01 & 61.60 & 31 & 2.68 & 513.31 & 3.56 & 4.12 & 3.29 & 4.17 & 2.67 \\
\hline-0.1 & 15.36 & 6.51 & 341.11 & 6.83 & 0.87 & 0.45 & 19.34 & 0.33 & 1.80 & 1.23 & 0.86 & 0.45 \\
\hline-0.4 & 25.67 & 60.03 & 5500.96 & 58.23 & 1.5 & 2.48 & 323.73 & 4.52 & 2.42 & 3.70 & 1.54 & 2.48 \\
\hline 0.2 & 9.73 & 19.56 & 363.52 & 22.36 & 0.58 & 1.56 & 77.31 & 0.73 & 1.45 & 2.09 & 0.59 & 1.56 \\
\hline 0.3 & 8.96 & 26.96 & 3059.43 & 23.88 & 0.53 & 0.88 & 176.96 & 2.20 & 1.64 & 2.52 & 0.54 & 0.89 \\
\hline-0.5 & 80.13 & 10.49 & 8314.62 & 19.79 & 4.33 & 0.92 & 509.83 & 0.62 & 4.09 & 1.69 & 4.18 & 0.90 \\
\hline 0.1 & 15.35 & 1.48 & 341.28 & 2.75 & 0.83 & 0.10 & 20.17 & 0.14 & 1.80 & 0.60 & 0.81 & 0.10 \\
\hline 0.4 & 25.68 & 20.48 & 5499.68 & 16.02 & 1.49 & 0.59 & 311.50 & 1.51 & 2.51 & 1.73 & 1.50 & 0.60 \\
\hline 0.3 & 9.60 & .14 & 77 & .75 & 0.53 & 1.14 & 31 & 8.46 & 1.62 & 1.87 & 0.51 & 1.13 \\
\hline
\end{tabular}

There also exists one case of which $d=-0.2$, whose $e_{f, F A}^{2}$ is larger than $e_{f, F B}^{2}$, but the corresponding $e_{f, F A}^{2}$ is smaller than $e_{f, F B}^{2}$. This is because that both the frequency responses of the filters and the signal spectrums are not flat. For example, when the peak frequency response error $\delta_{f}^{2}$ occurs at the frequency where the magnitude of the signal component is very small, the resultant time domain error is small; in this case, the value $e_{f}^{2}$, which is the integral of $\delta_{f}^{2}$ without any weighting, does not directly reflect the time domain error. A more accurate and fairer comparison is to evaluate the integral of weighted frequency response errors, which is actually the frequency domain error energy of the output signal, given as

$$
\hat{e}_{f}^{2}(d)=\frac{1}{\pi} \int_{0}^{a}\left|X_{i}\left(e^{j \omega}\right) \delta_{f}(\omega, d)\right|^{2} d \omega
$$


Table 4.6

$e_{f}^{2}, e_{g}^{2}$ GIVEN IN (4.18), $\hat{e}_{f}^{2}, \hat{e}_{g}^{2}$ GIVEN IN (4.20) AND (4.21), OF $F C$ AND $F D$, AND THE TIME DOMAIN ERRORS $\epsilon_{m}$ AND $e_{t}^{2}$ FOR CHIRP INPUT SIGNAL.

\begin{tabular}{|c|c|c|c|c|c|c|c|c|c|c|c|c|}
\hline \multirow{3}{*}{$d$} & \multicolumn{8}{|c|}{ Frequency Domain Error } & \multicolumn{4}{|c|}{ Time Domain Error } \\
\hline & \multicolumn{2}{|c|}{$e_{f}^{2}(d) \times 10^{6}$} & \multicolumn{2}{|c|}{$e_{g}^{2}(d) \times 10^{3}$} & \multicolumn{2}{|c|}{$\hat{e}_{f}^{2}(d) \times 10^{4}$} & \multicolumn{2}{|c|}{$\hat{e}_{g}^{2}(d)$} & \multicolumn{2}{|c|}{$\epsilon_{m} \times 10^{2}$} & \multicolumn{2}{|c|}{$e_{t}^{2} \times 10^{4}$} \\
\hline & \begin{tabular}{|l|}
$F C$ \\
\end{tabular} & $F D$ & $F C$ & $F D$ & $F C$ & $F D$ & $F C$ & $F D$ & $F C$ & $F D$ & $F C$ & $F D$ \\
\hline 0.1 & 55 & & & & & & & & & & & \\
\hline & & & & & 63 & & & & & & c & 0.00 \\
\hline & & & & & & & & & & & & \\
\hline 0 & 46 & & & & 874.4 & .2 & 42 & & 1. & & 8 & 350 \\
\hline & & & & & & & & & & & & \\
\hline & 39 & & & & & & 50 & & & & & 1.0 \\
\hline 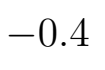 & 22 & & & & & & 23 & & & & ( & 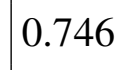 \\
\hline & & & & & & & & & & & & 46 \\
\hline & & & & & 2 & & 9 & & 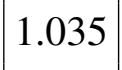 & & 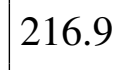 & 14 \\
\hline . & 8 & & & & & & 0 & & & & c & 0.4 \\
\hline 0 & & & & & & & & & & & 21 & 1.4 \\
\hline & & & 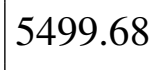 & & & & .50 & & 2.51 & 1.73 & 1.50 & 0.6 \\
\hline 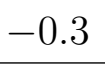 & 64 & & 97 & 0.054 & 1.260 & .31 & 225 & 0.044 & .0 & .189 & 1.322 & 11.3 \\
\hline
\end{tabular}

and

$$
\hat{e}_{g}^{2}(d)=\frac{1}{\pi} \int_{0}^{a}\left|X_{i}\left(e^{j \omega}\right) \delta_{g}(\omega, d)\right|^{2} d \omega
$$

where $X_{i}\left(e^{j \omega}\right)$ denotes the frequency spectrum of the signal segment processed by the VFD filters of a particular $d$ and $a=0.8 \pi$.

Such evaluated frequency domain errors of Pair 1 filters $(F A$ and $F B)$ and Pair 2 filters $(F C$ and $F D)$ are also given in Table 4.5 and 4.6, respectively. Comparing $\hat{e}_{f}^{2}$ and $\hat{e}_{t}^{2}$, it can be seen that, $e_{t}^{2}$ is consistent with $e_{f}^{2}$, and the later one is determined by $\left|X\left(e^{j \omega}\right)\right|^{2}$ and $\delta_{f}^{2}$. Obviously, this is a verification of Parsevals theorem. It is noted that significantly smaller $\delta_{g}(\omega, d), e_{g}^{2}(d)$ and $\hat{e}_{g}^{2}(d)$ of $F B$ and $F D$ have not shown slight effect to the time domain error. 
Table 4.7

THE FREQUENCY DOMAIN ERROR $\hat{e}_{f}^{2}, \hat{e}_{g}^{2}$ GIVEN IN (4.20) AND (4.21), OF $F A$ AND $F B$, AND THE TIME DOMAIN ERRORS $\epsilon_{m}$ AND $e_{t}^{2}$ FOR THE MUSIC INPUT SIGNAL.

\begin{tabular}{c|c|c|c|c||c|c|c|c}
\hline \hline \multirow{2}{*}{$d$} & \multicolumn{3}{|c|}{ Frequency Domain Error } & \multicolumn{3}{c}{ Time Domain Error } \\
\cline { 2 - 9 } & $\hat{e}_{f}^{2}(d) \times 10^{4}$ & \multicolumn{2}{|c|}{$\hat{e}_{g}^{2}(d)$} & \multicolumn{2}{c}{$\epsilon_{m} \times 10^{2}$} & \multicolumn{2}{c}{$e_{t}^{2} \times 10^{4}$} \\
\cline { 2 - 9 } & $F A$ & $F B$ & $F A$ & $F B$ & $F A$ & $F B$ & $F A$ & $F B$ \\
\hline-0.3 & 0.236 & 2.402 & 216.0 & 2.842 & 0.826 & 0.862 & 0.245 & 2.424 \\
0.1 & 2.279 & 0.686 & 80.36 & 2.780 & 0.924 & 0.412 & 2.287 & 0.701 \\
-0.4 & 1.542 & 19.49 & 1266 & 12.25 & 1.249 & 1.909 & 1.539 & 19.53 \\
0.3 & 0.045 & 0.097 & 3.255 & 0.500 & 0.846 & 1.303 & 0.045 & 0.102 \\
0.5 & 3.982 & 5.438 & 508.2 & 4.977 & 2.126 & 1.698 & 4.002 & 5.453 \\
-0.2 & 0.251 & 0.954 & 143.5 & 1.980 & 0.738 & 0.782 & 0.273 & 0.985 \\
-0.5 & 0.257 & 0.419 & 52.75 & 0.089 & 2.110 & 0.872 & 0.256 & 0.423 \\
-0.1 & 1.244 & 0.566 & 26.30 & 4.915 & 0.923 & 0.635 & 1.244 & 0.570 \\
0.2 & 0.094 & 0.345 & 26.38 & 0.712 & 0.748 & 1.078 & 0.102 & 0.351 \\
0.4 & 0.324 & 0.425 & 317.0 & 2.845 & 1.305 & 1.594 & 0.335 & 0.452 \\
\hline
\end{tabular}

\subsubsection{Real World Signal}

A music "Jingle bells" is also tested by the VFD filters to show that the relation between the frequency domain and time domain holds for deterministic wide band signals.

Since the sampling rate of the music is $44.1 \mathrm{kHz}$, the data computation is very huge if the whole music signal is processed. Hence, in this test, 1 second duration (41400 data samples) of the music signal is taken for the test to reduce the data computation, where the Fourier transform of the data is illustrated in Figure 4.3.

To make sure all the 41400 data samples are processed by the VFD filters $F C$ and $F D$, the simulation is conducted in the similar way the parameter $d$ is randomly nonrepeatablely varying among the values $\pm 0.1, \ldots, \pm 0.5$, where the duration of the signal segment for each delay value is fixed at 4,140 . The actual order of the random selection of $d$ is given in Table 4.7 and 4.8. Since the signal spectrum is not flat, $\hat{e}_{f}^{2}$ and $\hat{e}_{g}^{2}$ are also used to measure the integrated frequency errors and group delay errors, which are 
Table 4.8

THE FREQUENCY DOMAIN ERROR $\hat{e}_{f}^{2}, \hat{e}_{g}^{2}$ GIVEN IN (4.20) AND (4.21), OF FC AND FD, AND THE TIME DOMAIN ERRORS $\epsilon_{m}$ AND $e_{t}^{2}$ FOR THE MUSIC INPUT SIGNAL.

\begin{tabular}{c|c|c|c|c||c|c|c|c}
\hline \hline \multirow{3}{*}{$d$} & \multicolumn{3}{|c||}{ Frequency Domain Error } & \multicolumn{3}{c}{ Time Domain Error } \\
\cline { 2 - 10 } & $\hat{e}_{f}^{2}(d) \times 10^{4}$ & \multicolumn{2}{c||}{$\hat{e}_{g}^{2}(d)$} & \multicolumn{2}{|c|}{$\epsilon_{m} \times 10^{2}$} & \multicolumn{2}{c}{$e_{t}^{2} \times 10^{4}$} \\
\cline { 2 - 10 } & $F C$ & $F D$ & $F C$ & $F D$ & $F C$ & $F D$ & $F C$ & $F D$ \\
\hline-0.3 & 0.116 & 1.742 & 0.043 & 0.017 & 0.039 & 0.141 & 0.120 & 1.737 \\
0.1 & 4.933 & 15.34 & 1.448 & 0.139 & 0.065 & 0.113 & 4.928 & 15.29 \\
-0.4 & 0.263 & 5.944 & 0.090 & 0.038 & 0.015 & 0.071 & 0.260 & 5.940 \\
0.3 & 15.80 & 15.96 & 3.255 & 0.241 & 0.528 & 0.520 & 15.88 & 16.02 \\
0.5 & 2803 & 1364 & 457.9 & 13.25 & 2.015 & 1.406 & 2807 & 1366 \\
-0.2 & 0.210 & 2.226 & 0.075 & 0.018 & 0.021 & 0.069 & 0.225 & 2.234 \\
-0.5 & 0.011 & 0.228 & 0.002 & 0.003 & 0.014 & 0.064 & 0.010 & 0.225 \\
-0.1 & 0.522 & 2.019 & 0.068 & 0.015 & 0.020 & 0.038 & 0.525 & 2.038 \\
0.2 & 6.719 & 10.31 & 1.504 & 0.131 & 0.023 & 0.024 & 6.728 & 10.39 \\
0.4 & 6.796 & 3.870 & 9.134 & 0.491 & 1.295 & 0.893 & 6.812 & 3.891 \\
\hline
\end{tabular}

tabulated in Tables 4.7 and 4.8.

From Tables 4.7 and 4.8, it can be found that significantly small group delay errors of the VFD filters $F B$ and $F D$ do not shown any impact on the time domain errors neither.

\subsection{Summary}

This chapter investigated the optimization criteria for the design of VFD filters when VFD filters are used to estimate the instantaneous samples. The analysis and simulation results show that the traditional frequency response error is the criterion to be minimized in order to minimize the time domain estimation errors when instantaneous samples are concerned. However, it must be emphasized that the group delay certainly is the measurement of envelop delay, and the group delay error of VFD filters may also have an impact in other applications that were not considered in this chapter. 


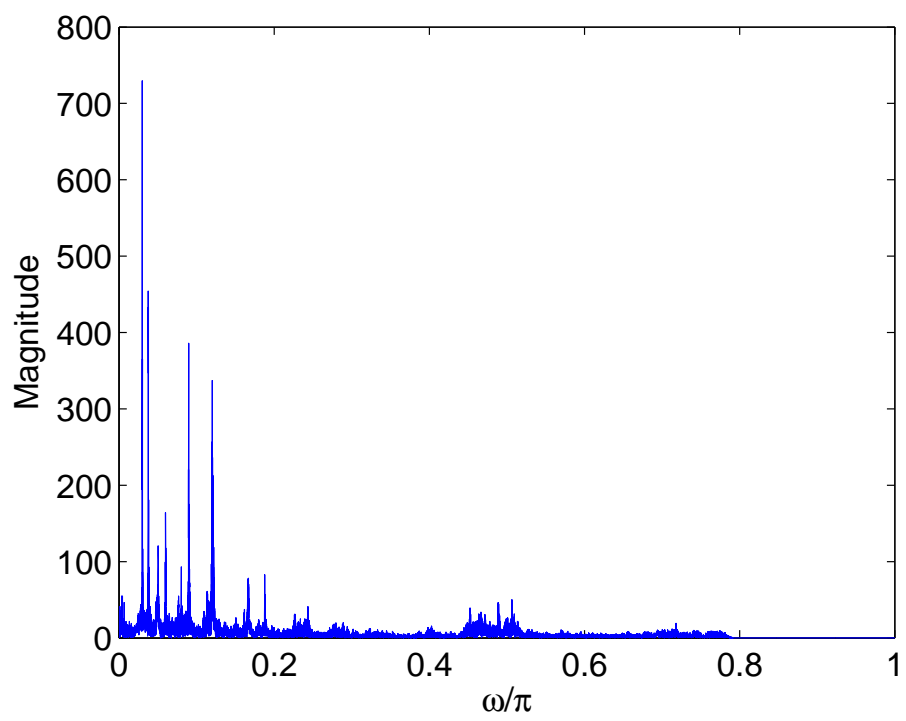

Figure 4.3. The frequency spectrum of the data samples of the music for test. 


\section{Chapter 5}

\section{Fast Filter Bank Approach for the Design of Variable Fractional Delay Filters}

Variable fractional delay (VFD) filters realized by polynomial based finite impulse response (FIR) filters [33-36,38-71] have been very popular due to the real-time update of coefficient values, accurate control of variable frequency characteristics and regular implementation patterns since 1988 when the Farrow structure [48] was introduced. Other VFD techniques include the weighted Lagrange interpolators [68, 132] and CORDIC rotations [133]. In this chapter, a filter bank approach is proposed for the design of VFD filters. The basic idea of the approach is to split the full band signals into subbands and each subband is respectively shifted by a phase which is determined by the required fractional delay. The overall fractional delay is achieved by combining all the subbands. The design examples show that the VFD can be accurately approximated by the proposed filter bank approach.

However, it has to be emphasized that the proposed filter bank approach requires more hardware cost than the existing techniques $[65,70,88]$ for the design of VFD filters. The purpose of the proposed technique is to provide a design technique of VFD filters that can be modified easily and effectively to design VBFD filters. The design of VBFD filters making use of the proposed VFD filters is present in Chapter 6. The combination 
of Chapter 5 and 6 is a complete presentation of the proposed design technique of VBFD filters. However, if two chapters are merged into a single chapter, it is too long to present. That is the reason that the design technique of VFD filters is presented standalone in this chapter, although its hardware cost is higher. Meanwhile, due to the same reason, the design examples in this chapter are arbitrarily chosen (because anyway the hardware cost of the proposed approach is higher than the existing techniques to achieve the same specifications). The two examples are chose in such a way to show that the proposed technique can realize the VFD filters no matter the filter specifications are relaxed or stringent.

The remaining of the chapter is organized as follows. Section 5.1 presents the principle and structure of the filter bank for the design of VFD filter. The implementation and computational complexity of the proposed VFD filter are also discussed in Section 5.1. The optimization of the prototype filter in the FFB is presented in Section 5.2. The design procedure of VFD filters is given in Section 5.3 and the design examples are shown in Section 5.4 to illustrate the efficiency of the proposed technique.

\subsection{Proposed Mixed-radix Fast Filter Bank}

In this section, a mixed-radix fast filter bank approach is proposed for the design of VFD filter, i.e., the filter is to approximate the ideal frequency response of

$$
\hat{H}\left(e^{j \omega}, d\right)=e^{-j \omega d}, 0 \leq \omega \leq \omega_{p}<\pi
$$

where $d$ is the variable fractional delay which varies in between -0.5 and 0.5 for a frequency range $\left[0, \omega_{p}\right]$ as illustrated in Section 2.4. The proposed technique can incorporate the variable bandedge conveniently into the VFD filter to achieve the simultaneously tuning of bandedge and fractional delay; the design of the simultaneously tunable bandedge and fractional delay filters are presented in Chapter 6. 


\subsubsection{Problem Formulation}

Ideally, if a full band signal $X\left(e^{j \omega}\right)$ is decomposed into fine subbands $X_{n}\left(e^{j \omega_{n}}\right)$, each band containing a signal with a single frequency $\omega_{n}$, i.e.,

$$
X\left(e^{j \omega}\right)=\sum_{n} X_{n}\left(e^{j \omega_{n}}\right)
$$

the fractional delay of $X_{n}\left(e^{j \omega_{n}}\right)$ can be achieved by multiplying the signal with a phase shift $e^{-j \omega_{n} d}$. The fractional delay of the original signal $X\left(e^{j \omega}\right)$ is obtained by

$$
Y\left(e^{j \omega}\right)=\sum_{n} X_{n}\left(e^{j \omega_{n}}\right) \cdot e^{-j \omega_{n} d}
$$

where $Y\left(e^{j \omega}\right)$ is the frequency spectrum of the delayed output.

However, practical signals are not able to be decomposed into subbands containing single frequency and the number of frequency components generally is infinite. In this section, a practical technique where signals are decomposed into a finite number of subbands is proposed to realize the variable fractional delay.

First, the full spectrum of the input signal is split by an $N$-band filter bank into $N$ uniform bands in the frequency range $[-\pi, \pi]$ centered at $\frac{2 \pi n}{N}$ respectively, for $-\left(\frac{N}{2}-\right.$ $1) \leq n \leq \frac{N}{2}$. The frequency response of each band is denoted as $H_{n}\left(e^{j \omega}\right)$. The output of each band is then multiplied respectively by a phase shift $e^{-j \frac{2 \pi}{N} n d}$. It can be expected that the center frequency component of each band has been shifted by a precise phase for a fractional delay $d$, while the phase shifts of the other frequency components are deviated from the ideal ones, if these bands are examined individually. However, if the bands are designed in such a manner that the superposition of adjacent bands results in phase shifts close to the ideal shifts at all frequency grids, the overall fractional delay is obtained by combining all the shifted subband signals (except band $\frac{N}{2}$. (The reason is explained in Section 5.1.3). Thus, the frequency response of the overall system can be represented as,

$$
H\left(e^{j \omega}, d\right)=\sum_{n=-\left(\frac{N}{2}-1\right)}^{\frac{N}{2}-1} H_{n}\left(e^{j \omega}\right) \cdot e^{-j \frac{2 \pi}{N} n d} .
$$




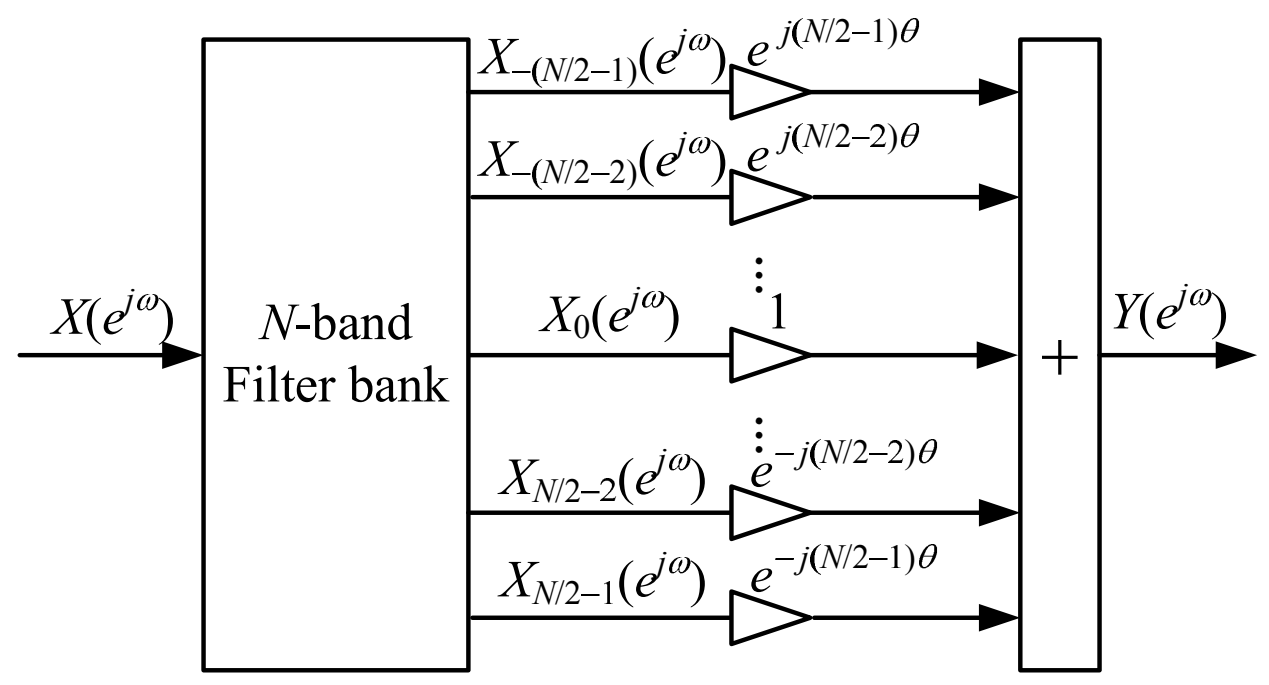

Figure 5.1. The conceptual diagram of the filter bank approach for the design of VFD filters, where $\theta=\frac{2 \pi}{N} d$.

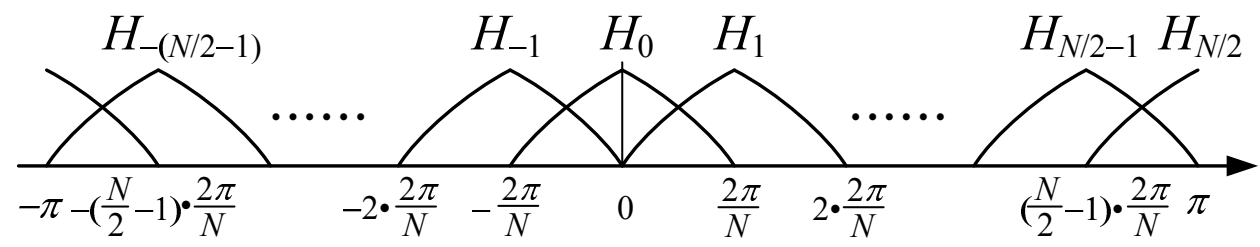

Normalized frequency $\omega$

Figure 5.2. Illustration of a filter bank with uniform bands of triangular frequency responses.

The conceptual diagram of the proposed technique is shown in Figure 5.1.

\subsubsection{Design of Filter Banks}

Intuitively, filter banks with triangular frequency response as shown in Figure 5.2 for each band may approximate the ideal fractional delay $\hat{H}\left(e^{j \omega}, d\right)$. Before an optimization problem is formulated in Section 5.2 for the design of a prototype filter, this section first proposes an efficient strategy to construct the filter bank based on a given prototype filter. To design the triangular filter bank with low computational complexity, based on the original FFB illustrated in Section 2.8.2, a mixed-radix fast filter bank (FFB) is 


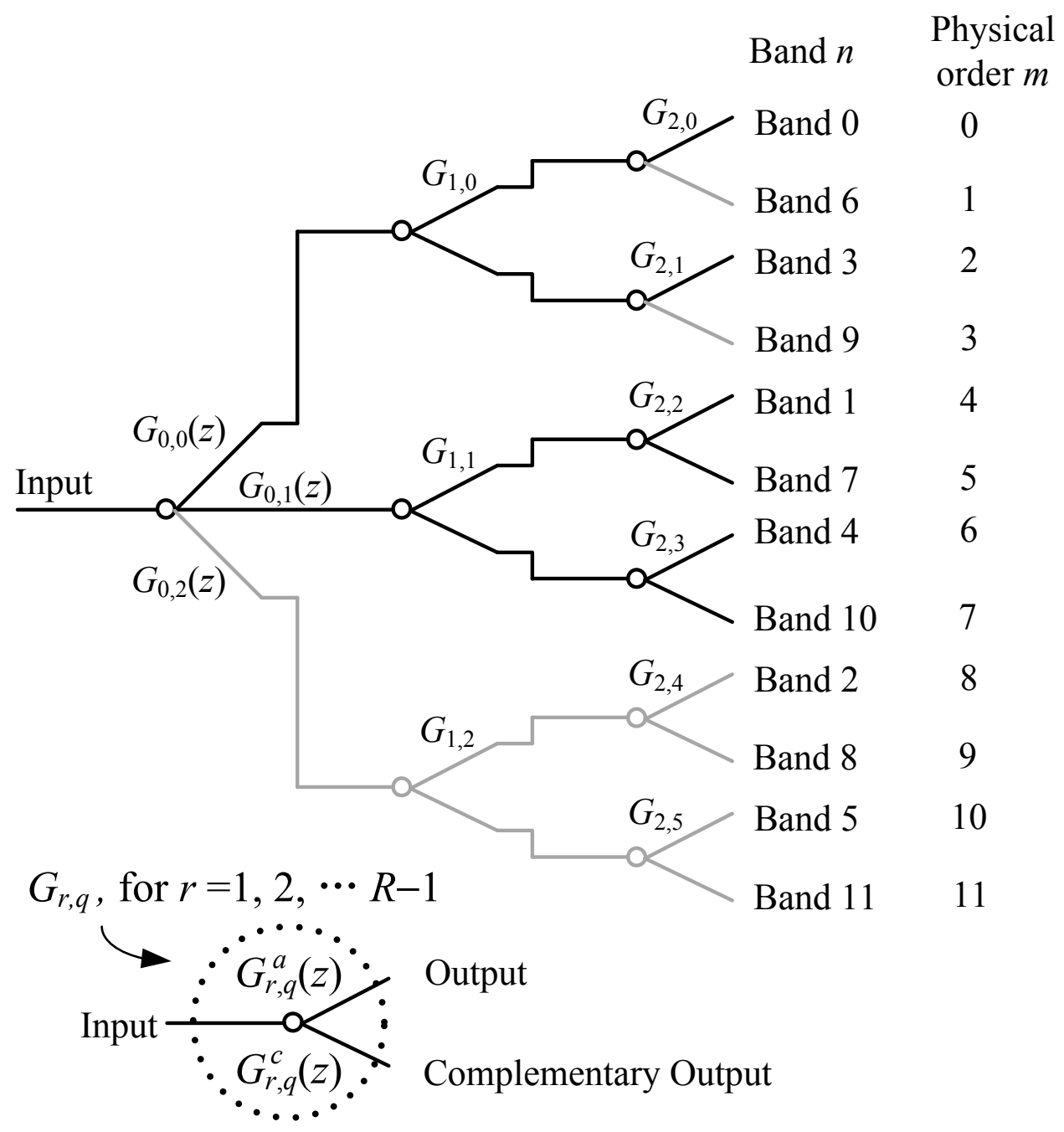

Figure 5.3. The structure of a 3-stage mixed-radix (3/2) FFB.

proposed. The filter structure of a 3-stage mixed-radix FFB is shown in Figure 5.3. In the first stage (stage 0), 3 filters split the full band signal (in normalized frequency $[0,2 \pi]$ ) into 3 channels, as shown in Figures 5.4(b)-(d); the 3 filters are compressed and frequency shifted versions of a prototype filter, whose frequency response is shown in Figure 5.4(a). Instead of the 2 complementary half-band filters used in the traditional FFB, 3 filters are used for the following reason: to split a full band signal into triangular bands using 2 filters, the frequency response of the prototype lowpass triangular filter extends its passband edge to $\pi$, which is adjacent to its own image in frequency range of $[\pi, 2 \pi]$, 
(a)

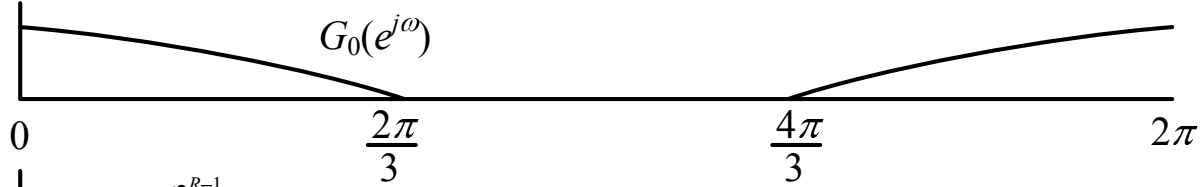

(b)

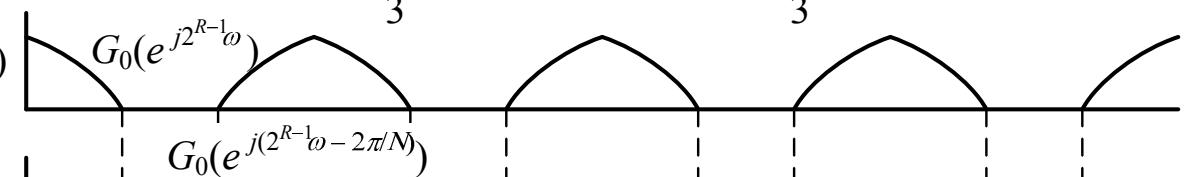

(c)

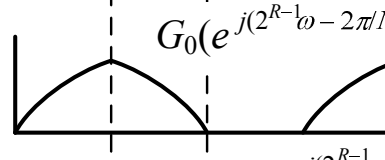

(d)

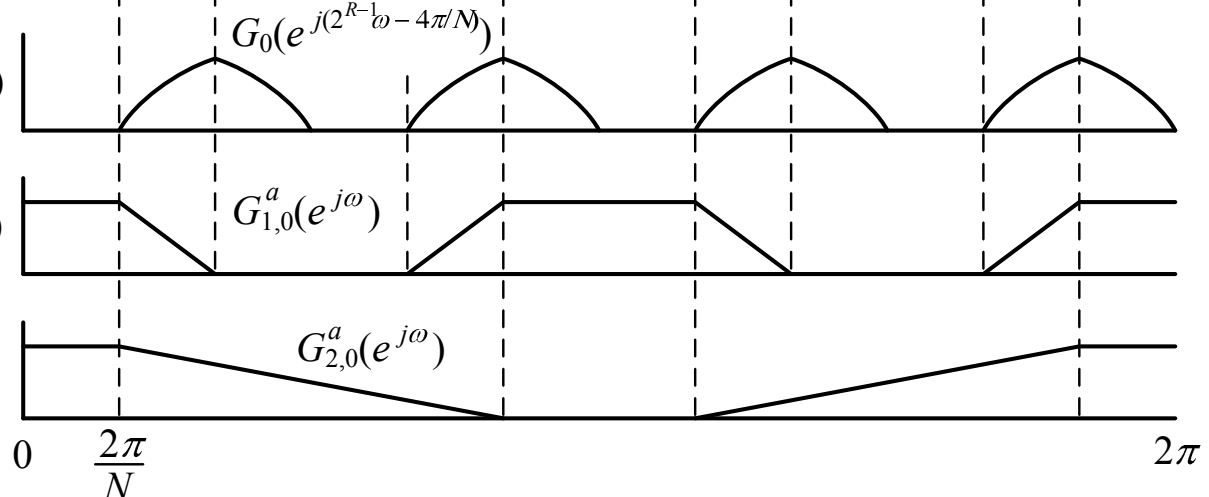

Figure 5.4. The frequency response of (a) the prototype filter $G_{0}\left(e^{j \omega}\right)$, and its compressed and shifted versions of (b) $G_{0}\left(e^{j 2^{R-1} \omega}\right)$, (c) $G_{0}\left(e^{j\left(2^{R-1} \omega-\frac{2 \pi}{N}\right)}\right)$, and (d) $G_{0}\left(e^{j\left(2^{R-1} \omega-\frac{4 \pi}{N}\right)}\right)$. The frequency response of (e) $G_{1,0}^{a}\left(e^{j \omega}\right)$ and (f) $G_{2,0}^{a}\left(e^{j \omega}\right) . \quad N=3 \times 2^{R-1}$ for an $R$-stage mixed-radix (3/2) FFB. In this example, $R=3$.

and hence after compression the bands in the same channel cannot be extracted using the subsequent filter stages without causing serious distortion. Three is the minimum number of filters that can split full band signal into triangular bands, which can be further extracted by subsequent filter stages. Once the full band signal is split into 3 channels, in the subsequent stages, each channel is further split into 2 channels (see Figure 5.3) as that in the original FFB. Since the first stage of the proposed FFB splits the signal into 3 channels, the overall FFB proposed is a mixture of radix-3 and radix- 2 structure. An $R$ stage mixed-radix (3/2) FFB generates $N$ channels in total, where $N=3 \times 2^{R-1}$.

The three filters of stage 0 , denoted as $G_{0,0}(z), G_{0,1}(z)$ and $G_{0,2}(z)$, are obtained by replacing each $z$ in a prototype filter $G_{0}(z)$ by $e^{-j \frac{2 \pi q}{N}} z^{2^{R-1}}$, for $q=0,1,2$, i.e.,

$$
G_{0, q}(z)=G_{0}\left(e^{-j \frac{2 \pi q}{N}} z^{2^{R-1}}\right), \text { for } q=0,1,2
$$


where, $G_{0}(z)$ is the $z$-transform transfer function of the prototype filter. The optimization of the prototype filter $G_{0}(z)$ is presented in Section 5.2.

In the subsequent stage $r$ for $r=1,2, \ldots, R-1, G_{r, q}$ for $q=0,1, \ldots, 3 \times 2^{r-1}-1$, represents a pair of complementary filters, $G_{r, q}^{a}(z)$ and $G_{r, q}^{c}(z) . G_{r, q}^{a}(z)$ is obtained by replacing each $z$ in a prototype lowpass half-band filter $G_{r}^{a}(z)$ by $e^{-j \frac{2 \pi \widetilde{q}}{N}} z^{2^{R-r-1}}$, i.e.,

$$
G_{r, q}^{a}(z)=G_{r}\left(e^{-j \frac{2 \pi \widetilde{q}}{N}} z^{2^{R-r-1}}\right)
$$

where $\widetilde{q}$ is the digit reverse version of $q$ in $R-1$ digits with mixed radices 3 and 2 . More specifically, $q$ is represented as an $(R-1)$-digit mixed-radix number where the most significant digit is of radix 3 , while the other digits are of radix $2 ; \widetilde{q}$ thus is obtained by reversing the $R-1$ digits of $q$, and $\widetilde{q}$ is also an $(R-1)$-digit mixed-radix number where the least significant digit is of radix 3 , while the other digits are of radix 2 .

The complimentary filter $G_{r, q}^{c}(z)$ is related to $G_{r, q}^{a}(z)$ by

$$
G_{r, q}^{c}(z)=1-G_{r, q}^{a}(z)
$$

Two examples of the frequency responses of the subsequent filter stage of $G_{1,0}^{a}\left(e^{j \omega}\right)$ and $G_{2,0}^{a}\left(e^{j \omega}\right)$ are illustrated in Figures 5.4(e) and 5.4(f). The function of these complimentary filter pairs is to extract individual bands from each channel, and therefore, the passband and stopband edges of the prototype half band filter $G_{r}^{a}(z)$ for stage $r$, denoted as $\omega_{p}^{r}$ and $\omega_{s}^{r}$, can be determined as,

$$
\omega_{p}^{r}=\frac{2 \pi}{N} \cdot 2^{R-r-1}
$$

and

$$
\omega_{s}^{r}=\pi-\omega_{p}^{r}
$$

In the above descriptions of (5.5)-(5.7), the filters are all assumed to be non-causal. Causality can be reinstated if necessary by delaying the non-causal impulse response by an appropriate number of samples. 
The $N$ bands obtained in this technique are in the order of $n=\widetilde{m}$, where $\widetilde{m}$ is the digit reverse version of $m$ in $R$ digits with mixed-radices 3 and 2, and $m$ is the physical order from top to down (corresponding to 0 to $N-1$ ) in the filter structure shown in Figure 5.3.

The $N$ bands generated using the proposed mixed-radix FFB are bands 0 to $(N-1)$ in the frequency range $[0,2 \pi]$. Due to the periodic property of frequency response, the band $n$ for $n \geq \frac{N}{2}$ in frequency range $[\pi, 2 \pi]$ is equivalent to band $(n-N)$ (negative) in the frequency range $[-\pi, 0]$, as those shown in Figure 5.2. In the following of this chapter, band $n$ and band $(n-N)$ are used interchangeably to refer to the band $n$ for $n \geq \frac{N}{2}$.

\subsubsection{Implementation Issue}

From Figure 5.1, it can be seen that band $n$, for $-\left(\frac{N}{2}-1\right) \leq n \leq \frac{N}{2}-1$, is to be shifted by a phase $e^{-j \frac{2 \pi}{N} n d}$. Since the phases to be shifted at $\pi$ and $-\pi$ for fractional delay are different, the approximation of the ideal fractional delay at frequency $\pi$ is not achievable. Therefore, only are bands from band $-\left(\frac{N}{2}-1\right)$ to band $\left(\frac{N}{2}-1\right)$ shifted and combined to achieve the overall fractional delay for frequency range $\left[-\left(\frac{N}{2}-1\right) \frac{2 \pi}{N},\left(\frac{N}{2}-1\right) \frac{2 \pi}{N}\right]$ at most. In addition, the output of band $H_{n}\left(e^{j \omega}\right)$, denoted as $X_{n}\left(e^{j \omega}\right)$, for $1 \leq n \leq \frac{N}{2}-1$, is a complex conjugate of $X_{-n}\left(e^{j \omega}\right)$; the sum of the phase shifted $X_{n}\left(e^{j \omega}\right)$ and $X_{-n}\left(e^{j \omega}\right)$ is equivalent to twice of the real part of $X_{n}\left(e^{j \omega}\right) e^{-j \frac{2 \pi}{N} n d}$ or $X_{-n}\left(e^{j \omega}\right) e^{j \frac{2 \pi}{N} n d}$, i.e.,

$$
\begin{aligned}
X_{n}\left(e^{j \omega}\right) e^{-j \frac{2 \pi}{N} n d}+X_{-n}\left(e^{j \omega}\right) e^{j \frac{2 \pi}{N} n d} & =2 \mathfrak{R}\left(X_{n}\left(e^{j \omega}\right) e^{-j \frac{2 \pi}{N} n d}\right) \\
& =2 \mathfrak{R}\left(X_{-n}^{*}\left(e^{j \omega}\right) e^{-j \frac{2 \pi}{N} n d}\right)
\end{aligned}
$$

where, $\mathfrak{R}(\cdot)$ finds the real part of '.' and $X^{*}$ is the complex conjugate of $X$. Therefore, in

actual implementation, only $\frac{N}{2}$ bands are shifted and summed together. In each conjugate pair of $X_{n}\left(e^{j \omega}\right)$ and $X_{-n}\left(e^{j \omega}\right)$, either $X_{n}\left(e^{j \omega}\right) e^{-j \frac{2 \pi}{N} n d}$ or $X_{-n}^{*}\left(e^{j \omega}\right) e^{-j \frac{2 \pi}{N} n d}$ is used. The computational complexity is the factor to determine which of them is used. For example, 


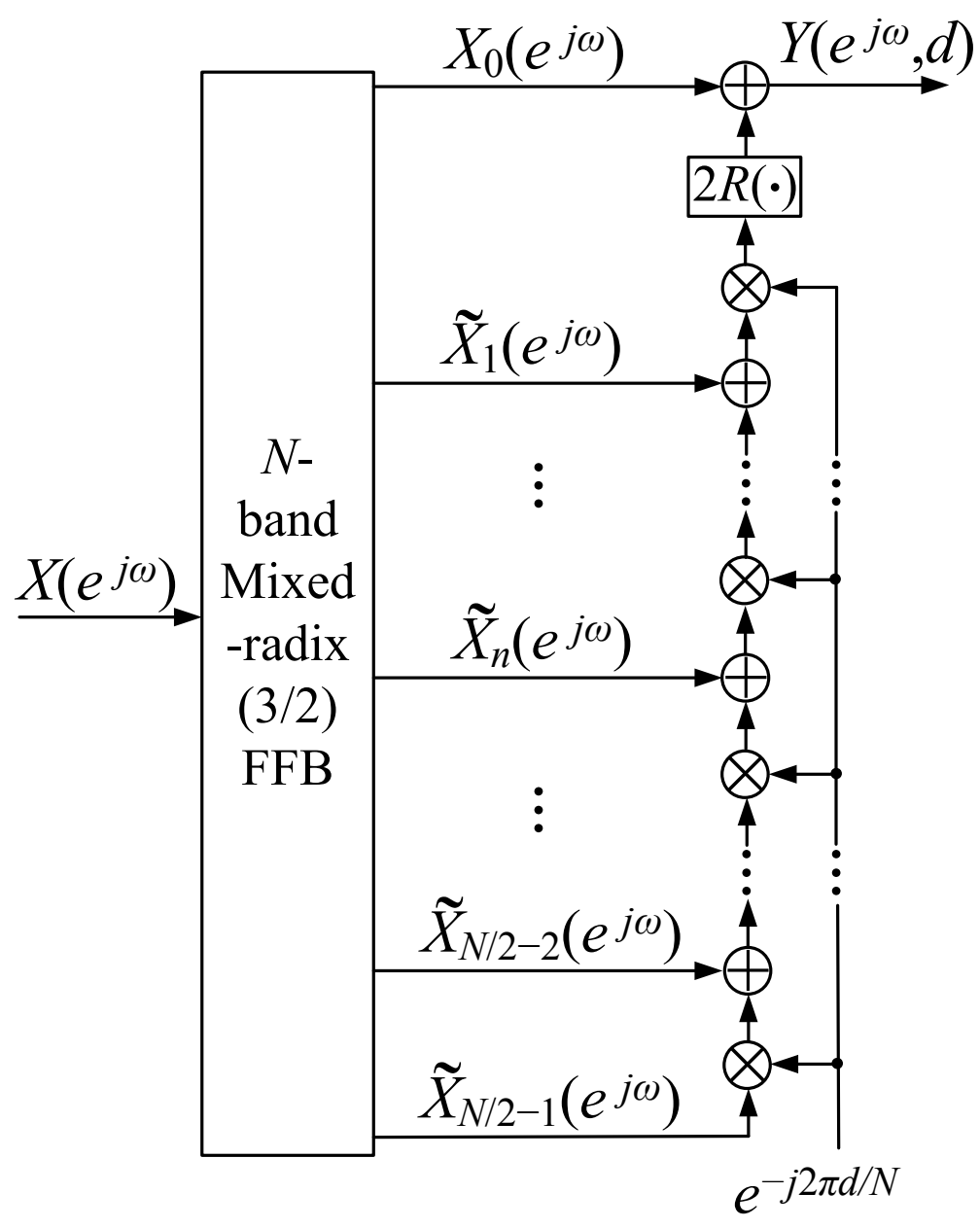

Figure 5.5. The refined implementation structure for VFD filter, where $\tilde{X}_{n}\left(e^{j \omega}\right)$ represents $X_{n}\left(e^{j \omega}\right)$ or $X_{-n}^{*}\left(e^{j \omega}\right)$ for $n=1, \ldots, \frac{N}{2}-1$.

in the 3-stage mixed-radix FFB shown in Figure 5.3, the bands led to by black lines, i.e., bands $0,3,1,7,4$ and 10 are used, while those led to by grey lines need not to be computed at all, such that the number of branches to be computed is minimized. For an $R$-stage $(R \geq 3)$ mixed-radix (3/2) FFB, the bands used are band $n=\widetilde{m}$, for

$$
m \in\{0\} \cup_{r=1}^{R-2}\left\{2^{r}, 2^{r}+1, \ldots, 2^{r}+2^{r-1}-1\right\} \cup\left\{2^{R-1}, 2^{R-1}+1, \ldots, 2^{R}-1\right\} .
$$

In general, it can be seen that the filters in the last branch from $G_{0,2}(z)$ need not be computed.

The conceptual diagram in Figure 5.1, thus, can be refined to the implementation 
structure of VFD filter shown in Figure 5.5, where $\widetilde{X}_{n}\left(e^{j \omega}\right)$ represents either $X_{n}\left(e^{j \omega}\right)$ or $X_{-n}^{*}\left(e^{j \omega}\right)$, to minimize the computational complexity.

\subsubsection{Computational Complexity}

In the operation of the proposed VFD filters, multiplications dominate the computational complexity. Two types of multiplications are involved in the structure in Figure 5.5. One is the multiplication for the mixed-radix FFB, and the other the phase shift terms.

According to the bands selected for further processing in Section 5.1.3, stage 0 has 3 filters, but only 2 of them have to be computed. Stage 1 includes 3 pairs of filters where only 2 pairs are actually used. Furthermore, the filters in each pair are complimentary and thus only one filter in each pair requires multipliers. Stage $r$ involves $3 \times 2^{r-1}+1$ filters for $r=2, \ldots, R-2$, and $3 \times 2^{r-1}$ filters for $r=R-1$, but in both cases $3 \times 2^{r-2}+1$ filters actually have to be computed. Among these filters, there are three types of filters in view of the computational complexity: type A filter has real filter coefficients and real input signal, type B has complex filter coefficients and real input signal, whereas both filter coefficients and input signal are complex for type $\mathrm{C}$ filter. To generate one output sample, the number of real multiplications for the filters of type $\mathrm{A}, \mathrm{B}$, and $\mathrm{C}$ are once, twice and three times of the number of the nontrivial filter taps, respectively. Note that the multiplication of two complex numbers can be realized using three real multipliers in the price of one more real adder (in total three real adders) [134]. Stage 0 has 1 type A filter and 1 type B filter, stage 1 has 1 type A filter and 1 type $\mathrm{C}$ filter, while all the other stages have 1 type A filter, 1 type B filter, and $3 \times 2^{r-2}-1$ type C filters, for Stage $r$.

Let the orders of the prototype filters of $G_{r}(z)$, for $r=0,1, \ldots, R-1$ be $K_{r}$. Since $G_{r}(z)$ for $r=1,2, \ldots, R-1$ are half band filters, $K_{r}=4 \kappa_{r}+2$, where $\kappa_{r}$ is a nonnegative integer. For $G_{0}(z)$, even order is used to avoid half delay. Thus, for an $R$ stage FFB, the overall number of real multiplications, denoted as $C_{F F B}$, to compute $\frac{N}{2}$ output 
samples, one for each band, is given by:

$$
\begin{aligned}
C_{F F B} & =\left(\frac{K_{0}+2}{2}\right) \times 3-1+\left(\frac{K_{1}+2}{4}\right) \times 4-1 \\
& +\sum_{r=2}^{R-1}\left(\frac{K_{r}+2}{4}\left(9 \times 2^{r-2}\right)-3 \times 2^{r-2}\right) .
\end{aligned}
$$

where the real-value middle taps of the complex filters are considered.

The implementation of the phase shifting $e^{-j \frac{2 \pi}{N} d}$ in time domain is to multiply the signal, say $x(l)$, by $e^{j \frac{2 \pi}{N} d T_{s}}=\cos \left(\frac{2 \pi}{N} d T_{s}\right)+j \sin \left(\frac{2 \pi}{N} d T_{s}\right)$. To shift each band the corresponding phase, $\frac{N}{2}-1$ complex multipliers are used as shown in Figure 5.5. In addition to that, the values of $\sin \left(\frac{2 \pi}{N} d T_{s}\right)$ and $\cos \left(\frac{2 \pi}{N} d T_{s}\right)$ have to be computed before they can be multiplied into the structure. Standard look-up table and angular decomposition [31] can be used to obtain high frequency resolution sine and cosine values using 8 multipliers. Alternative techniques of digital frequency synthesis may also be found in [135]. Therefore, the number of real multipliers for phase shift, denoted by $C_{\text {phase }}$, is given by

$$
C_{\text {phase }}=3\left(\frac{N}{2}-1\right)+8+1=\frac{3 N}{2}+6
$$

where the term ' 1 ' before the last equal sign is to calculate $\frac{2 \pi}{N} d T_{s}$.

In all these multipliers, those used to calculate the sine and cosine values, and used to compute the phase shifting are general multipliers (i.e., multipliers carrying two variable operands); the others are constant multipliers (i.e., multipliers carrying a constant operand and a variable operand). Therefore, for the total $C_{F F B}+C_{\text {phase }}$ multipliers, $C_{F F B}+1$ are constant multipliers and $C_{\text {phase }}-1$ are general multipliers.

\subsection{Optimization Design of Prototype Filter $G_{0}(z)$ in FFB}

In this section, an optimization technique for the 0th stage prototype filter is proposed by taking all the errors introduced by the FFB into consideration, to reduce the overall error.

Equations (5.6) and (5.7) show that $G_{r, q}^{a}\left(e^{j \omega}\right)$ and $G_{r, q}^{c}\left(e^{j \omega}\right)$ are a pair of complementary filters, constructed from a prototype filter $G_{r}\left(e^{j \omega}\right)$. The prototype filters $G_{r}\left(e^{j \omega}\right)$, 
for $r=1, \ldots, R-1$, are half band filters with the passband and stopband edges given in (5.8) and (5.9). In the improved optimization technique, first, these half band filters are designed independently with ripple tolerance $\delta_{r}=\delta / 2^{r}$ for $r=1, \ldots, R-1$, where $\delta$ is the allowable accumulated ripple of the filters of all stages. The determination of $\delta$ from the specifications $\delta_{m}$ and $\delta_{p}$ is given in Section 5.5.2. It can be seen that the ripple of the $r$ th stage prototype half band filter is chosen to be half of that of $(r-1)$ th stage, because the transition width of $r$ th stage filter is wider than that of $(r-1)$ th stage. Such choices of ripples may make the orders of all half band prototype filters at the similar level, and the computational complexity of the overall filter designed in such a way is low according to our design experiences.

Once the prototype filters of $G_{r}\left(e^{j \omega}\right)$ for $r=1, \ldots, R-1$ are designed, the next step is to design $G_{0}\left(e^{j \omega}\right)$.

The $R$-stage $N$-band mixed-radix (3/2) FFB structure used for the synthesis of VFD is shown in Figure 5.3, where the 0th stage splits the signals into 3 channels and subsequently each channel is split into two in the $r$ th stage, for $r=1, \ldots, R-1$. Figure 5.3 shows bands $r$ for $r=0, \ldots, N-1$ with $N=12$. As stated in the end of Section 5.1.2, the bands $\frac{N}{2}, \frac{N}{2}+1, \ldots, N-1$ in Figure 5.3 are equivalent to the bands $-\frac{N}{2},-\frac{N}{2}+1, \ldots,-1$ in Figure 5.1 due to the periodic frequency response, and they are interchangeable in the remainder of the chapter for the expression convenience.

In the 0th stage in Figure 5.3, $G_{0, q}$, short for $G_{0, q}\left(e^{j \omega}\right)$, is a compressed and shifted version of a prototype filter $G_{0}\left(e^{j \omega}\right)$, where $q$ indicates the index of filters in the stage, given by

$$
G_{0, q}\left(e^{j \omega}\right)=G_{0}\left(e^{-j \frac{2 \pi q}{N}} e^{-j 2^{R-1} \omega}\right), \text { for } q=0,1,2
$$

Assuming an even order $G_{0}(z)$ with order $K_{0}$ and symmetric impulse response $g(k)$, for 
$k=-\frac{K_{0}}{2}, \ldots, 0, \ldots, \frac{K_{0}}{2}$, the zero-phase frequency response of $G_{0}\left(e^{j \omega}\right)$ is given by

$$
G_{0}\left(e^{j \omega}\right)=\sum_{k=-\frac{K_{0}}{2}}^{\frac{K_{0}}{2}} g(k) e^{-j k \omega}=\mathbf{L}(\omega) \mathbf{g}
$$

where

$$
\mathbf{L}(\omega)=\left[1,2 \cos (\omega), 2 \cos (2 \omega) \ldots, 2 \cos \left(\frac{K_{0}}{2} \omega\right)\right]
$$

and

$$
\mathbf{g}=\left[g(0), g(1), \ldots, g\left(\frac{K_{0}}{2}\right)\right]^{T} .
$$

Substituting (5.15)-(5.17) into (5.14), $G_{0, q}\left(e^{j \omega}\right)$ can be written in the matrix form as

$$
G_{0, q}\left(e^{j \omega}\right)=\mathbf{L}\left(2^{R-1}\left(\omega-q \frac{2 \pi}{3}\right)\right) \mathbf{g}, \text { for } q=0,1,2
$$

Hence, the frequency response of channel $n$, denoted as $H_{n}\left(e^{j \omega}\right)$, is given as

$$
H_{n}\left(e^{j \omega}\right)=G_{0, q(n, 0)}\left(e^{j \omega}\right) \Pi_{r=1}^{R-1} G_{r, q(n, r)}^{*}\left(e^{j \omega}\right),
$$

where $G_{r, q(n, r)}^{*}\left(e^{j \omega}\right)$ denotes $G_{r, q}^{a}\left(e^{j \omega}\right)$ when $\left\lfloor\frac{n}{3 \times 2^{r-1}}\right\rfloor$ is even and $G_{r, q}^{c}\left(e^{j \omega}\right)$ when $\left\lfloor\frac{n}{3 \times 2^{r-1}}\right\rfloor$ is odd, and $q(n, r)$ is given as

$$
q(n, r)= \begin{cases}\bmod (n, 3) & \text { for } r=0,1 \\ \tilde{s}(n, r)+\bmod (n, 3) 2^{r-1} & \text { otherwise. }\end{cases}
$$

In (5.20), $\tilde{s}$ stands for the bit reverse version of $s$ in $r-1$ bits, where $s$ is given as

$$
s=\left\lfloor\frac{\bmod \left(n, 3 \times 2^{r-1}\right)}{3}\right\rfloor
$$

with $\lfloor x\rfloor$ representing the largest integer not larger than $x$.

Therefore, the overall frequency response of the VFD filter, synthesized using the $\mathrm{FFB}$, is given as

$$
H\left(e^{j \omega}, d\right)=\sum_{n=-\frac{N}{2}+1}^{\frac{N}{2}-1} G_{0, q(0,0)}\left(e^{j \omega}\right) \Pi_{r=1}^{R-1} G_{r, q(n, r)}^{*}\left(e^{j \omega}\right) e^{-j \frac{2 \pi}{N} n d}
$$


Substituting (5.18) into (5.22), the frequency response of the overall filter can be written in the matrix form as

$$
H\left(e^{j \omega}, d\right)=\mathbf{T}\left(e^{j \omega}, d\right) \mathbf{g}
$$

where $\mathbf{T}\left(e^{j \omega}, d\right)$ is given by

$$
\mathbf{T}\left(e^{j \omega}, d\right)=\sum_{k=-1}^{1} t_{k}\left(e^{j \omega}, d\right) \mathbf{L}\left(2^{R-1}\left(\omega-k \frac{2 \pi}{3}\right)\right) .
$$

In (5.24), the parameter $t_{k}\left(e^{j \omega}, d\right)$ is given by

$$
t_{0}\left(e^{j \omega}, d\right)=\Pi_{r=1}^{R-1} G_{r, q(0, r)}^{*}\left(e^{j \omega}\right)
$$

and

$$
t_{k}\left(e^{j \omega}, d\right)=\sum_{n=1}^{\frac{N}{2}-1} \Pi_{r=1}^{R-1} G_{r, q(n, r)}^{*}\left(e^{j \omega}\right) e^{-j \frac{2 \pi}{N} n k d}
$$

for $k= \pm 1$.

The frequency response error of the overall VFD filter, denoted as $J(\mathbf{g})$, is thus obtained as

$$
J(\mathbf{g})=\left|H\left(e^{j \omega}, d\right)-e^{-j \omega d}\right|=\left|\mathbf{T}\left(e^{j \omega}, d\right) \mathbf{g}-e^{-j \omega d}\right|
$$

The prototype filter coefficients of stage 0 can be obtained in the minimax sense of frequency response error as

$$
\text { minimize: } \max _{0<\omega \leq \omega_{p},-0.5 \leq d \leq 0.5} \delta_{f}(\omega, d)=J(\mathbf{g})=\left|\mathbf{T}\left(e^{j \omega}, d\right) \mathbf{g}-e^{-j \omega d}\right|
$$

The optimization problem in (5.28) can be solved by a general convex programming package [136] as well. To optimize $G_{0}\left(e^{j \omega}\right)$ by solving (5.28), the filter order of $G_{0}\left(e^{j \omega}\right)$, $K_{0}$, has to be determined first. For the allowable accumulated ripple $\delta, K_{0}$ can be estimated based on a lowpass filter with transition bandwidth $\frac{2 \pi}{3}$ and a ripple tolerance $\delta_{0}=\frac{\delta}{2^{R-1}}$. 


\subsection{Design Parameters}

In this section, the determination of the stage number $R$ and the estimation of the prototype filter orders of the FFB when using the improved optimization technique are discussed.

\subsubsection{Determination of the stage number of the FFB}

Two aspects of the filter specifications determine the number of stages of the FFB. The first specification is the passband width $\omega_{p}$. Since the band $N / 2$ is always discarded in the synthesis of VFD, the upper band of the frequency range with controlled VFD $\omega_{p}$ is given by $\pi-\frac{2 \pi}{N}$. When the band $N / 2$ is discarded, the sidelobe of band $N / 2-1$ cannot be properly canceled. Hence, the frequency approximation errors increase dramatically when the frequency goes beyond $\pi-\frac{4 \pi}{N}$ and $\pi-\frac{2 \pi}{N}$. The plots of the maximum frequency response errors versus $\omega_{p}$ are shown in Figure 5.6 for $R=3,4,5$ and 6 , respectively. For example, for $R=3$, it can be found that the frequency response errors increase significantly when $\omega$ becomes larger than $\frac{2}{3} \pi$ and $\frac{5}{6} \pi$.

The second specification is the frequency response error requirements. In the working passband range $\left[0, \omega_{p}\right]$, the maximum frequency response errors decrease with the increasing of the number of stages $R$ and the decreasing $\omega_{p}$. For given passband edge and maximum magnitude and phase errors, the number of the FFB stage $R$ can be determined according to Figure 5.6.

\subsubsection{Determination of $\delta$ for the estimation of the Prototype Filter orders}

Once $R$ is determined, the values of $K_{r}$, for $r=0, \ldots, R-1$, for each stage has to be estimated to satisfy both the magnitude and phase specifications. As stated in Section 5.3 , the orders are based on an allowable accumulated ripple, $\delta$, of the filters of all stages. This chapter derives the expression of $\delta$ based on $\delta_{p}$ and $\delta_{m}$. 


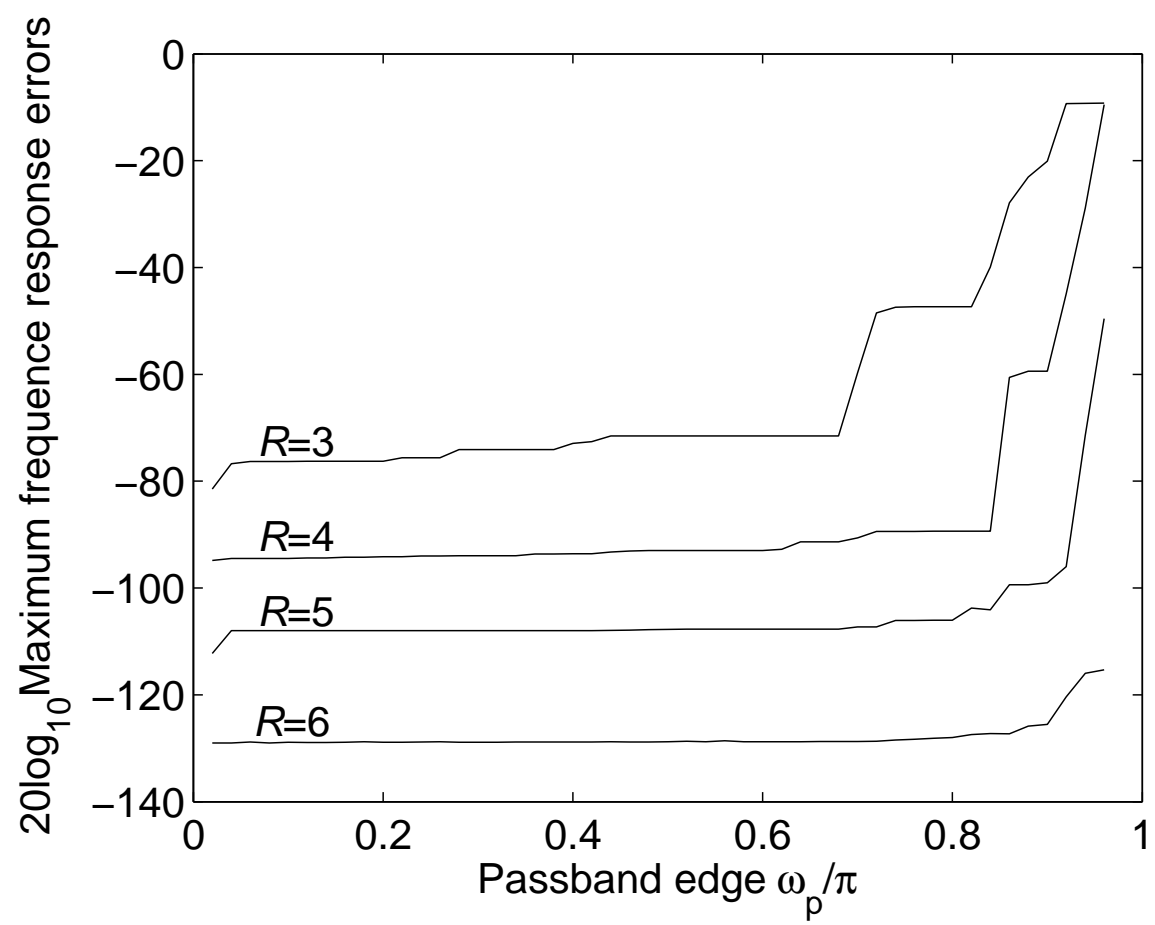

Figure 5.6. The maximum frequency response errors vs. the number of stages of the FFB $R$ and the passband edge $\omega_{p}$.

The principle of the filter bank approach for the synthesis of VFD is the superposition of the adjacent bands with phase shifts as shown in Figure 5.7(a). At an arbitrary frequency $\omega_{a}$, assume that $a_{k-1}$ and $a_{k}$ are the magnitudes of bands $H_{k-1}$ and $H_{k}$. The synthesis of the fractional delay is shown in Figure 5.7(b), where $\vec{a}_{k-1}$ and $\vec{a}_{k}$ are the vectors of $a_{k-1}$ and $a_{k}$ with the phase shift factors. Therefore, the phase difference $\beta$ of $\vec{a}_{k-1}$ and $\vec{a}_{k}$ is

$$
\beta=\frac{2 \pi d}{N}
$$

The vector sum of $\vec{a}_{k-1}$ and $\vec{a}_{k}$ is the magnitude and phase responses of the overall filter. Suppose $a_{k-1}$ and $a_{k}$ are the ideal magnitude responses so that the resultant $\frac{2 \pi(k-1) d}{N}+\theta$ is the ideal angle corresponding to the fractional delay, i.e., there is no phase error. It satisfies

$$
\frac{a_{k}}{\sin \theta}=\frac{a_{k-1}}{\sin (\beta-\theta)}
$$




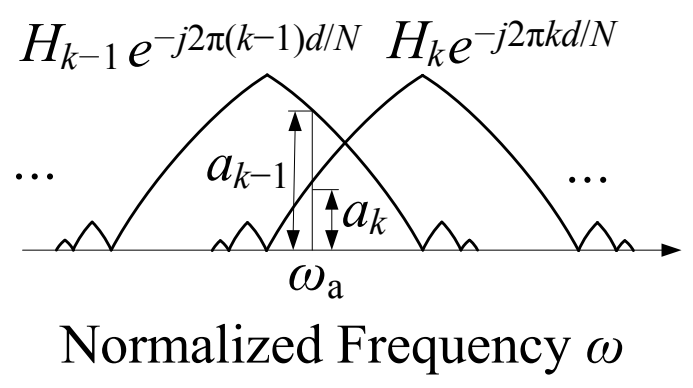

(a)

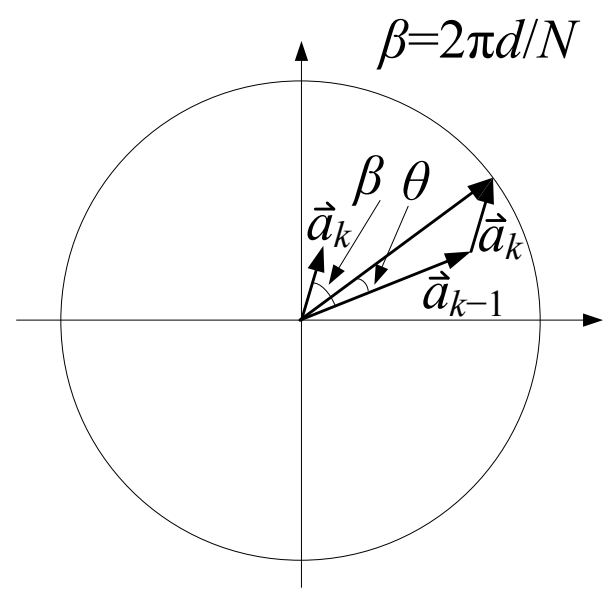

(b)

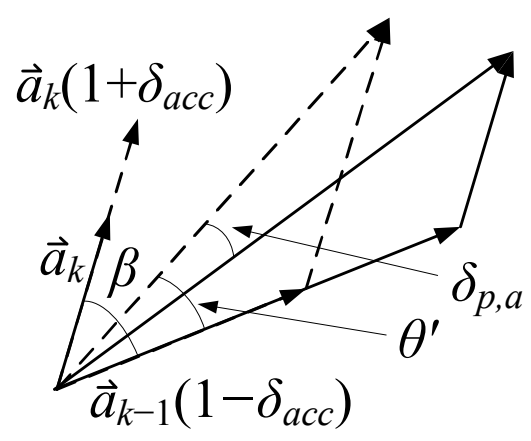

(c)

Figure 5.7. The principle of the synthesis of VFD using filter bank. (a) the frequency responses of two adjacent bands $k-1$ and $k$, (b) the vector representations of the superposition of the phase shifted signals and (c) the error effect. 
Substituting (5.29) into (5.30), (5.30) can be rewritten as

$$
\tan \theta=\frac{a_{k} \sin \frac{2 \pi d}{N}}{a_{k-1}+a_{k} \cos \frac{2 \pi d}{N}} .
$$

Equation (5.31) can be simplified as

$$
\theta \approx \tan \theta=\frac{a_{k} \sin \frac{2 \pi d}{N}}{a_{k-1}+a_{k} \cos \frac{2 \pi d}{N}},
$$

when $\theta$ is small for the case $N \geq 12(R \geq 3)$, and further simplified as

$$
\theta \approx a_{k} \frac{2 \pi d}{N}
$$

due to $a_{k-1}+a_{k} \approx 1$ for small $\theta$.

Assuming that the maximum accumulated magnitude error of the filters of all stages is $\delta_{a c c}$, in the worst case, $a_{k-1}$ and $a_{k}$ are deviated in the opposite direction from the ideal values, i.e., becoming $a_{k-1}\left(1-\delta_{a c c}\right)$ and $a_{k}\left(1+\delta_{a c c}\right)$, or vice versa. Figure 5.7(c) shows the case. Thus, the actual resultant phase is given by

$$
\theta^{\prime} \approx a_{k}\left(1-\delta_{a c c}\right) \frac{2 \pi d}{N}
$$

and the resultant phase error $\delta_{p, a}$ at $\omega_{a}$ is given as

$$
\delta_{p, a}=\theta-\theta^{\prime} \approx a_{k} \delta_{a c c} \frac{2 \pi d}{N}
$$

Since the maximum value of $a_{k}$ is 1 , given the maximum phase error $\delta_{p}$, the allowable maximum accumulated ripples of the filters of all stages, when considering the phase specification only, is given by

$$
\delta_{a c c}=\frac{\delta_{p}}{\frac{\pi}{N}} \text { when } d= \pm 0.5 .
$$

When considering both $\delta_{p}$ and $\delta_{m}$, the allowable accumulated magnitude errors, $\delta$, of the filters of all stages, is given by

$$
\delta=\min \left(\delta_{a c c}, \delta_{m}\right)=\min \left(\frac{\delta_{p}}{\frac{\pi}{N}}, \delta_{m}\right),
$$

for the estimation of the prototype filter orders in Section 5.3. 


\subsubsection{Summary of Design Steps}

The steps for the design of VFD filters using the improved optimization technique are summarized as follows:

1. For the given specifications of $\omega_{p}, \delta_{m}$ and $\delta_{p}$, the numbers of the FFB stages is determined according to Section 5.4.1;

2. The half band prototype filters $G_{r}\left(e^{j \omega}\right)$ for $r=1,2 \ldots, R-1$ are optimized according to the specifications given in the second paragraph of Section 5.3;

3. The order of the 0th stage prototype filter is determined according to the last paragraph of Section 5.3. And $G_{0}\left(e^{j \omega}\right)$ is optimized by solving the problem formulated in (5.33).

\subsection{Design Example}

In this section, two VFD filter examples are designed to illustrate the proposed filter bank technique in designing VFD filters with relax and stringent specifications, respectively.

\subsubsection{Example A}

The specifications of the VFD filter are given as follows:

$$
\begin{aligned}
& \omega_{p}=0.84 \pi \text { (passband edge); } \\
& \delta_{f}=5 \times 10^{-4} \text { (maximum magnitude error); } \\
& d \in[-0.5,0.5] \text { (fractional delay variation range). }
\end{aligned}
$$

To design VFD filters with the bandedge no less than $0.84 \pi$ and the maximal frequency response error no larger than $5 \times 10^{-4}$, a 4-stage FFB is required according to Figure 5.6, and $K_{0}=6, K_{1}=22, K_{2}=14$ and $K_{3}=10$ is desired according to the design steps in Section 5.3.3.

The frequency response, magnitude and phase errors of the design examples are illustrated in Figures 5.8. 

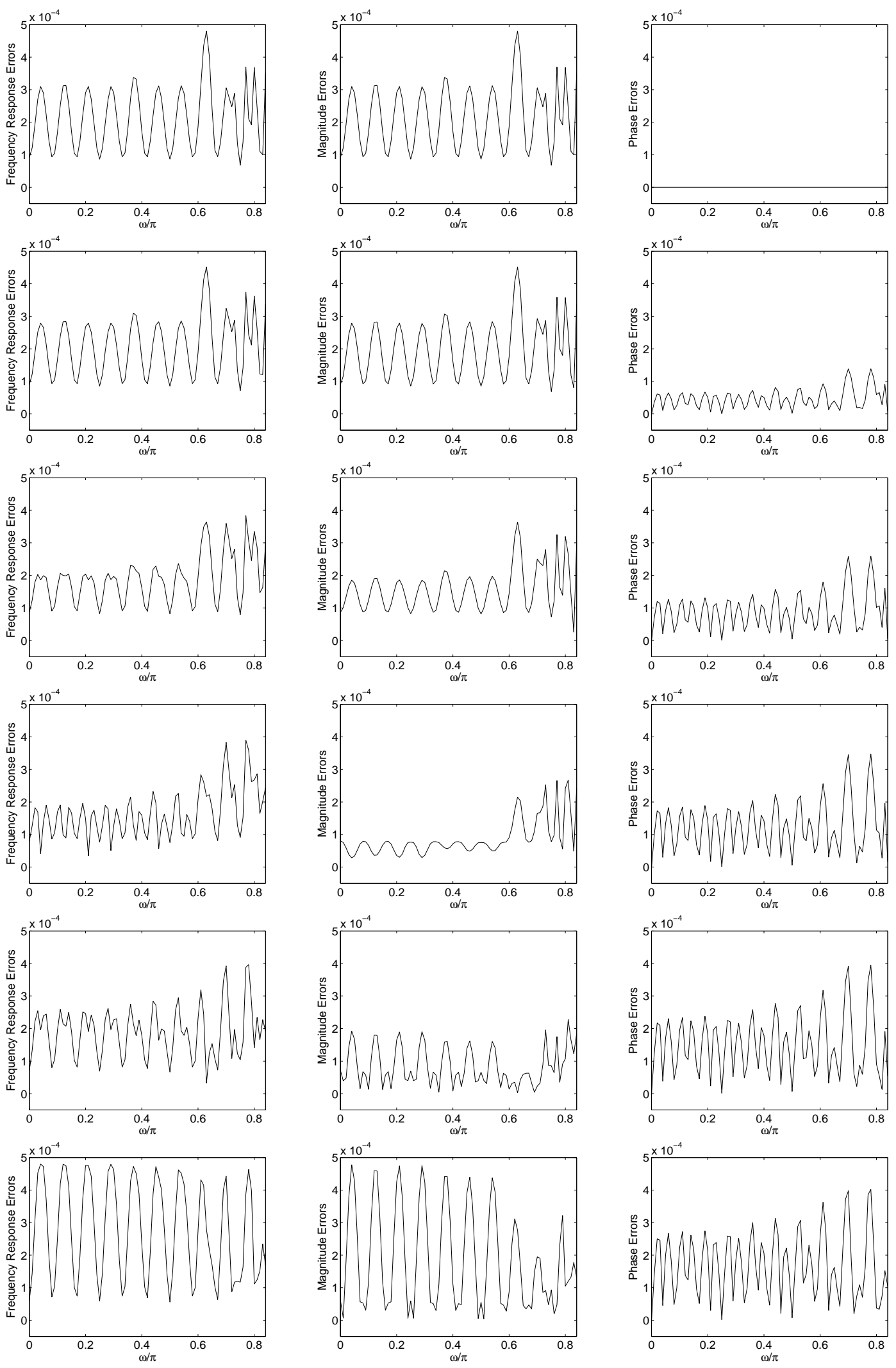

Figure 5.8. Example A: The frequency response errors (Left column), magnitude errors (Middle column) and phase errors (Right column) of the designed VFD filter for $d=$ $0, \pm 0.1, \ldots, \pm 0.5$ (rows from top to bottom). 
Table 5.1

THE COMPLEXITIES OF THE VFD FILTERS USING THE PROPOSED TECHNIQUE AND THE TECHNIQUES IN [65], RESPECTIVELY.

\begin{tabular}{c|c|c|c}
\hline \hline Technique & Multiplications & Additions & Group Delays \\
\hline The technique in [65] & 77 & 75 & 14 \\
\hline Proposed & 157 & 374 & 87 \\
\hline \hline
\end{tabular}

To meet the same specifications, the technique in [65] requires the filter order and the polynomial order 28 and 5, respectively. The computational complexities of the designs using the proposed technique and the technique in [65] are listed in Table 5.1.

\subsubsection{Example B}

The specifications of the VFD filter are cited from [70, 88], given as follows:

$$
\begin{aligned}
& \omega_{p}=0.95 \pi \text { (passband edge); } \\
& \delta_{f}=1 \times 10^{-3} \text { (maximum frequency response error); } \\
& d \in[-0.5,0.5] \text { (fractional delay variation range). }
\end{aligned}
$$

To design VFD filters with bandedge no less than $0.95 \pi$ and frequency response error no larger than $1 \times 10^{-3}$, a 5 -stage FFB can meet this requirement according to Figure 5.6, and $K_{0}=14, K_{1}=22, K_{2}=10, K_{3}=10$ and $K_{4}=6$ are desired. The frequency response, magnitude and phase errors of the VFD filter are illustrated in Figures 5.9.

The complexities of the design examples as well as the approximation errors are tabulated in Table 5.2, which reveals that the proposed technique could also achieve the VFD filter with ultra wide bandwidth, although its complexity is still high, compared with the recent techniques $[70,88]$. 

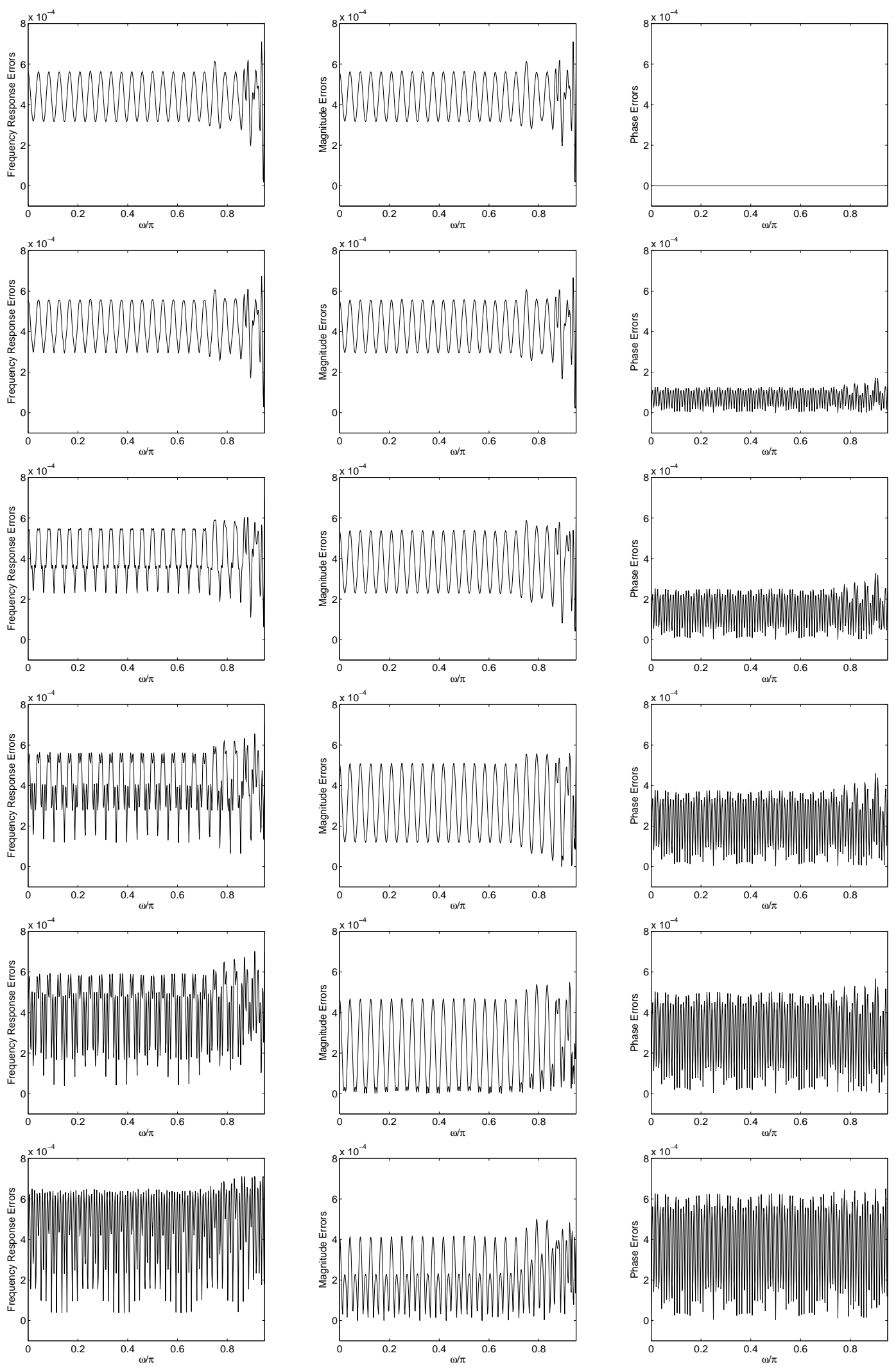

Figure 5.9. The frequency response (Left column), magnitude (Middle column) and phase errors (Right column) of the VFD filter designed by the proposed technique for $d=$ $0, \pm 0.1, \ldots, \pm 0.5$ (rows from top to bottom). 
Table 5.2

THE COMPLEXITY AND THE MAXIMUM FREQUENCY RESPONSE ERROR OF THE VFD FILTER USING THE PROPOSED TECHNIQUE, THE TECHNIQUES IN [70] AND [88], RESPECTIVELY.

\begin{tabular}{c|c|c|c|c}
\hline \hline Technique & Multiplications & Additions & Group Delays & $\delta_{f}(\mathrm{~dB})$ \\
\hline Hybrid, WLS [70] & 141 & 271 & 116 & -59.48 \\
\hline $\mathrm{LP}_{1}[88]$ & 64 & 117 & 82 & -61.57 \\
\hline $\mathrm{LP}_{-} \mathrm{RFM}_{1}[88]$ & 43 & 73 & 88 & -64.20 \\
\hline $\mathrm{LP}_{2}[88]$ & 58 & 108 & 84 & -61.63 \\
\hline $\mathrm{LP}_{-} \mathrm{RFM}_{2}[88]$ & 36 & 62 & 88 & -62.78 \\
\hline Proposed & 253 & 661 & 201 & -62.97 \\
\hline \hline
\end{tabular}

\subsection{Discussion}

The design examples in Section 5.5 show that the proposed techniques can realize VFD, but it does not show any advantages in terms of less complexity or approximation errors, compared with the techniques for designing VFD filters in $[65,70,88]$.

In fact, the objective of this chapter is to illustrate an innovative prototype idea for realizing VFD and validates its feasibility. Based on this principle, VBFD could be simply achieved as illustrated in Chapter 6.

\subsection{Summary}

This chapter proposed a mixed-radix FFB approach for the design of VFD filters. An optimization technique is presented to optimize the prototype filters of the FFB. Through the FFB, the input full band signals are split into several subbands. By shifting each subband a proper phase, the fractional delay is realized by combining necessary subbands. Design examples show that the proposed technique may design VFD filters for any given specifications. However, the computational and implementation complexities are higher than those achieved by the polynomial based approximation. As stated in the introduction of this chapter, the VFD filters based on the FFB approach are proposed to be used 
in the design of the VBFD filters in Chapter 6, and only are trivial extra computations required, while the existing techniques for the design of the VBFD filters may increase the hardware cost significantly. 


\section{Chapter 6}

\section{Fast Filter Bank Approach for the Design of Variable Bandedge and Fractional Delay Filters}

As reviewed in Chapters 2 and 5, the polynomial based Farrow structure has been widely employed in the synthesis of variable fractional delay (VFD) filters [33-36, 38-71]. When it comes to the digital filters with simultaneously and independently variable bandedge and fractional delay (VBFD), two-dimensional polynomial approximation is required [89-91]. Parallel form [89,90] and nested Farrow structure [91] were proposed to implement the two-dimensional polynomials [89-91], but the computational complexities of such structures are considerably high. An improved nested Farrow structure is proposed in [92], where each branch of the first level Farrow structure is cascaded with a fixed filter, such that the overall complexity is reduced.

In this chapter, based on the mixed-radix FFB approach proposed in Chapter 5 for the design of VFD filters, two approaches for the realization of VBFD filters are proposed. In the first approach, the bandwidth of the individual band of the FFB is designed to be not wider than the required transition band of the VBFD filter. In such a method, the VBFD filter can be realized by simply keeping the bands in the passband of the VBFD filter and discarding the bands out of the passband. However, the narrow band constraint of the FFB results in unnecessary computational and implementation complexity. In the second 
approach, band shaping filters are introduced to shape the last retained band to form the transition band of the variable filter, such that the bandwidth of the individual band may be relaxed to twice of the transition bandwidth of the VBFD filter. Although more than one shaping filters have to be implemented, for a particular passband edge variation, only one shaping filter is in use and hence the computational complexity incurred due to the band shaping is low. The overall computational complexity of the filter obtained using the second approach therefore is further reduced.

While the computational complexity may be reduced by the introduction of the shaping filters, the implementation complexity is increased if every band has to be cascaded with a shaping filter, since when the bandedge is varying, every band may become the last retained band. Further investigations show that all involved bands can share 3 shaping filters constructed from a prototype filter. By employing such a shaping filter sharing technique, the overall implementation complexity of the VBFD filter remains low.

The remainder of the chapter is organized as follows. Sections 6.1 and 6.2 propose the two techniques incorporating the variable bandedge into the VFDs, respectively. Computational and implementation complexities of the two proposed techniques are analyzed in Section 6.3. Section 6.4 gives the design procedure of VBFD filters and Section 6.5 presents three design examples to show the advantages of the proposed approaches.

\subsection{Approach 1: Band Combination for the Synthesis of VBFD}

As reviewed in Section 2.6, the design of VBFD filters is to optimize the filter such that the frequency response satisfies the constraints:

$$
\begin{aligned}
& || H\left(e^{j \omega}, \phi, d\right)|-1| \leq \delta_{m, p}, \text { for } \omega \in[0, \phi] \\
& \left|H\left(e^{j \omega}, \phi, d\right)\right| \leq \delta_{m, s}, \text { for } \omega \in[\phi+\Delta \omega, \pi] ; \\
& |\Phi(\omega, \phi, d)-(-d \omega)| \leq \delta_{\Phi}, \text { for } \omega \in[0, \phi] ;
\end{aligned}
$$


where $d$ and $\phi$ denote the variable fractional delay and passband edge, varying in the range $[-0.5,0.5]$ and $\left[\omega_{l}, \omega_{u}\right]$, respectively. $\Delta \omega$ is the transition bandwidth of the VBFD filter. An illustration of the specification refers to Figure 2.11.

Since the full band signal can be split into multibands by the FFB proposed in Chapter 5, these bands can be either included into the final response forming a passband or discarded becoming a stopband. The tuning of the bandedge, $\phi$, thus can be realized by varying the number of bands to be included. According to the technique proposed in Chapter 5, the fractional delay requirement is satisfied for frequencies up to $\omega_{m}$ as specified in (5.1). The maximum value of $\phi$ is thus bounded by $\omega_{p}$, i.e., $\omega_{u} \leq \omega_{p}$, such that the passband of the filter always satisfies the fractional delay requirement, whereas the fractional delay in the stopband is not cared. To ensure the continuously tuning of the bandedge by discretely varying the number of bands to be included in the passband, the bandwidth of the individual band $\left(\frac{4 \pi}{N}\right)$ in the FFB must be controlled.

When the variable bandedge is achieved by discarding the outputs of the bands out of the passband of the VBFD filter, the side lobes of the filter $G_{0, q\left(e^{j \omega}\right)}$ given in (5.14) and (5.18) for $q=0,1$ and 2, must be controlled during the optimization. This is because in the minimization of $J_{2}(\mathbf{g})$ in $(5.31)$, the side lobes of each band are compensated by other bands; when some bands are discarded in the synthesis of VBFD filters, the uncompensated side lobes may violate the filter specifications.

Figure 6.1 shows the frequency responses of bands $0, \ldots, \frac{N}{2}$ of the $R$-stage $N$-band FFB, denoted as $H_{0}, H_{1}, \ldots, H_{N / 2-1}$, respectively. In Figure 6.1, the side lobes of the bands are presented, where $\gamma$ is the width of the side lobes containing significant leakage energy. Generally, $\gamma$ is smaller than $\frac{\pi}{N}$. In Figure 6.1, the bandwidth of each band is $\frac{4 \pi}{N}$ and the center frequency of band $k$, denoted as $\omega_{k}$, is given as

$$
\omega_{k}=\frac{2 \pi k}{N}
$$

For a particular variation of the passband edge $\phi$ falling in the range $\left(\omega_{k-1}-\gamma, \omega_{k}-\gamma\right]$, 


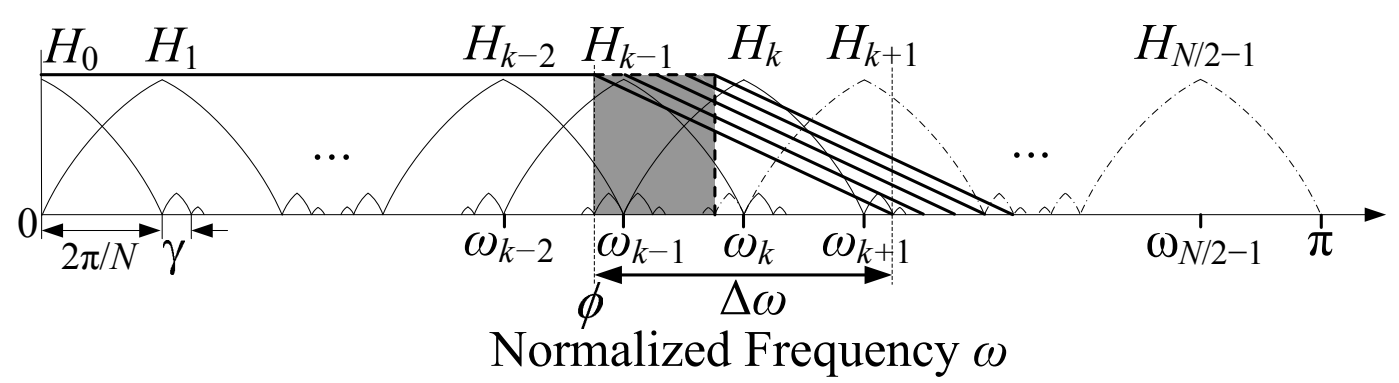

Figure 6.1. The structure of an $R$-stage $N$-band mixed-radix (3/2) FFB to synthesize the VBFD filter.

shown as the shadow region in Figure 6.1 , the outputs of bands $0,1 \ldots, k$ (in solid line) are summed to meet the passband requirement of the VBFD and the outputs of the other bands (in dash-dot line) are discarded to meet the stopband requirement of the VBFD. This requires a transition bandwidth of the VBFD, $\Delta \omega$, not smaller than $\frac{4 \pi}{N}+2 \gamma$, i.e.,

$$
\Delta \omega \geq \frac{4 \pi}{N}+2 \gamma
$$

otherwise, the main lobe and/or the side lobe of band $k$ may locate in the passband or stopband of the VBFD filter and violate the ripple tolerances.

Thus, for a given $\Delta \omega$ in the VBFD, according to (6.2), the FFB must split the full band signal into $N$ bands with $N \geq \frac{4 \pi}{\Delta \omega-2 \gamma}$. And when $\phi$ varies, the criteria to determine which bands are retained or discarded is, for band $k$, if $\phi>\omega_{k-1}-\gamma$, the band is kept; otherwise it is discarded. Furthermore, for given $\Delta \omega$ and $N$, according to (6.2), $\gamma$ has to be constrained to

$$
\gamma \leq \frac{\Delta \omega}{2}-\frac{2 \pi}{N}
$$

Since $\gamma$ is the width of the side lobe of the individual band $H_{n}\left(e^{j \omega}\right)$ in the FFB, translated back to the prototype filter $G_{0}\left(e^{j \omega}\right)$, the corresponding allowed width of the side lobe of $G_{0}\left(e^{j \omega}\right)$ is $2^{R-1} \gamma$. Thus, in the optimization of $G_{0}\left(e^{j \omega}\right)$, the ripples at the frequency beyond the passband and the allowed side lobe at $\omega$ for $\frac{2 \pi}{3}+2^{R-1} \gamma \leq \omega \leq \pi$ must be controlled.

Therefore, when the FFB is optimized to synthesize a VBFD, besides minimizing the objective function given in (5.28), additional constraints are applied to $G_{0}\left(e^{j \omega}\right)$ and the 


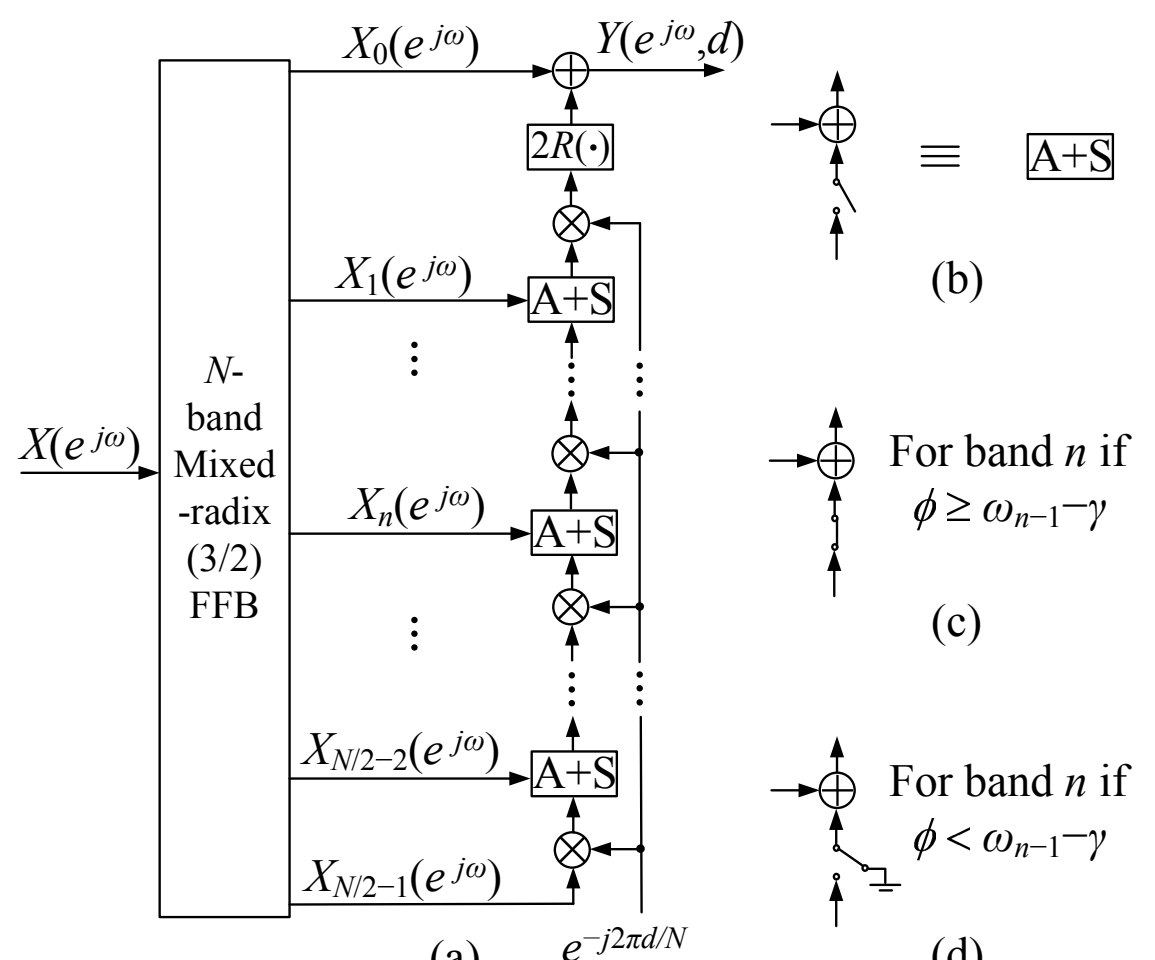

(a) $\quad e^{-j 2 \pi d / N}$

(d)

Figure 6.2. Structure of the variable filter. (a) Overall structure; (b) an "A+S" unit; (c) and (d) the switch status of the "A+S" unit for different bands, where $\tilde{X}_{n}\left(e^{j \omega}\right)$ represents $X_{n}\left(e^{j \omega}\right)$ or $X_{-n}^{*}\left(e^{j \omega}\right)$ for $n=1, \ldots, \frac{N}{2}-1$.

optimization problem becomes

$$
\begin{aligned}
& \text { minimize: } \max _{0<\omega \leq \omega_{u},-0.5 \leq d \leq 0.5} J_{2}(\mathbf{g})=\left|\mathbf{T}\left(e^{j \omega}, d\right) \mathbf{g}-e^{-j \omega d}\right|, \\
& \text { subject to: }-b \leq \mathbf{L}(\omega) \mathbf{g} \leq b, \text { for } \frac{2 \pi}{3}+2^{R-1} \gamma \leq \omega \leq \pi .
\end{aligned}
$$

where $b$ is the tolerance of the insignificant side lobes. According to the superposition principle, $b$ should be smaller than $\delta / 2^{R-1}$, where $\delta$ is the allowable accumulated magnitude error derived in Section 5.4.2. The optimization problem in (6.4) can be solved by the same convex programming package [136].

For the synthesis of the VBFD filter, the VFD structure in Figure 5.5 may be revised to Figure 6.2 to implement the proposed variable filters with simultaneously tunable fractional delay and bandedge. Since $X_{n}\left(e^{j \omega}\right)$ and $X_{-n}^{*}\left(e^{j \omega}\right)$ are interchangeable for $n=1, \ldots, \frac{N}{2}-1, X_{n}\left(e^{j \omega}\right)$ only is used in this chapter for expression convenience. 
In Figure 6.2, the $N / 2$ bands generated from the mixed-radix (3/2) FFB are fed into an "A+S"-unit-and-multiplier-chain. An "A+" unit is shown in Figure 6.2(b) consisting of an adder and a switch. The switch in the " $\mathrm{A}+\mathrm{S}$ " unit of band $n$ controls if the signals generated in the bands $n+1$ and onwards should be included into the final output or not. To achieve this, the condition to close or open the switch of band $n$ is if $\phi \geq \omega_{n-1}-\gamma$, as shown in Figures 6.2(c) and (d), i.e., when $\phi \geq \omega_{n-1}-\gamma$, the band $n$ is kept, otherwise it is discarded.

By controlling the connections of these switches in such a way, the $N / 2$ bands are either contributing to the passband or to the stopband of the final response.

\subsection{Approach 2: Band Shaping for the Synthesis of VBFD}

To synthesize the VBFD filter by combining the necessary bands of the FFB and discarding the remaining as discussed in Section 6.1, the transition bandwidth of the VBFD filter must be wider than $\frac{4 \pi}{N}+2 \gamma$ as shown in (6.2). To design the VBFD filter with narrower transition band, either $N$ is increased by increasing the number of stages of the FFB, or decreasing $\gamma$. However, either approach increases the computational complexity of the FFB significantly.

The basic idea of the approach is to employ 3 fixed multi-band over designed shaping filters to shape the bands of the FFB. The over designed shaping filters have sharper transition band than the required transition band of the VBFD filter. Thus, although the 3 fixed multi-band shaping filter only provide discontinuously variable bandedges, the continuously variable bandedge requirement of the VBFD filter can be satisfied.

In this section, a band shaping approach is introduced to the bands of the FFB for the synthesis of VBFD filters. 


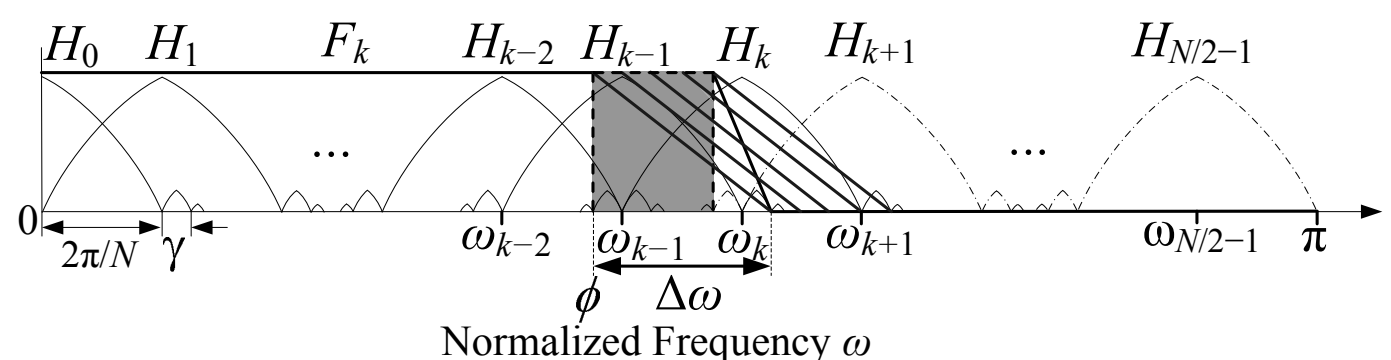

Figure 6.3. The structure of an $R$-stage $N$-band mixed-radix (3/2) FFB with a shaping filter $F_{k}\left(e^{j \omega}\right)$ for band $k$.

\subsubsection{Construction of Shaping Filters}

From Figure 6.1 and the same as in Figure 6.3, it can be seen that the constraint in (6.2) is due to the fact that when the variable passband edge $\phi$ falls in $\left(\omega_{k-1}-\gamma, \omega_{k}-\gamma\right]$, the entire or the major part of the last retained band $k$ is outside the passband of the VBFD filter; these part has to be constrained inside the transition band of the VBFD filter. If a lowpass shaping filter $F_{k}$ is cascaded to band $k$ to keep the frequency response up to $\omega_{k}-\gamma$ and mask out the frequency response $\omega_{k}+\gamma$ onwards, as shown in Figure 6.3, the transition bandwidth $\Delta \omega$ of the VBFD in (6.2) may be decreased to

$$
\Delta \omega \geq \frac{2 \pi}{N}+2 \gamma
$$

i.e., for the same $N$ and $\gamma$, sharper VBFD filter can be synthesized.

Given the passband variation range $\left[\phi_{\min }, \phi_{\max }\right]$, the band $k$ that may become the last retained band satisfies

$$
N_{L} \leq k \leq N_{H}
$$

where $N_{L}$ and $N_{H}$ denote the smallest and largest index of the band that may be the last retained band, given as

$$
N_{L}=\left\lceil\frac{N\left(\phi_{\min }+\gamma\right)}{2 \pi}\right\rceil
$$

and

$$
N_{H}=\left\lceil\frac{N\left(\phi_{\max }+\gamma\right)}{2 \pi}\right\rceil,
$$

respectively. 


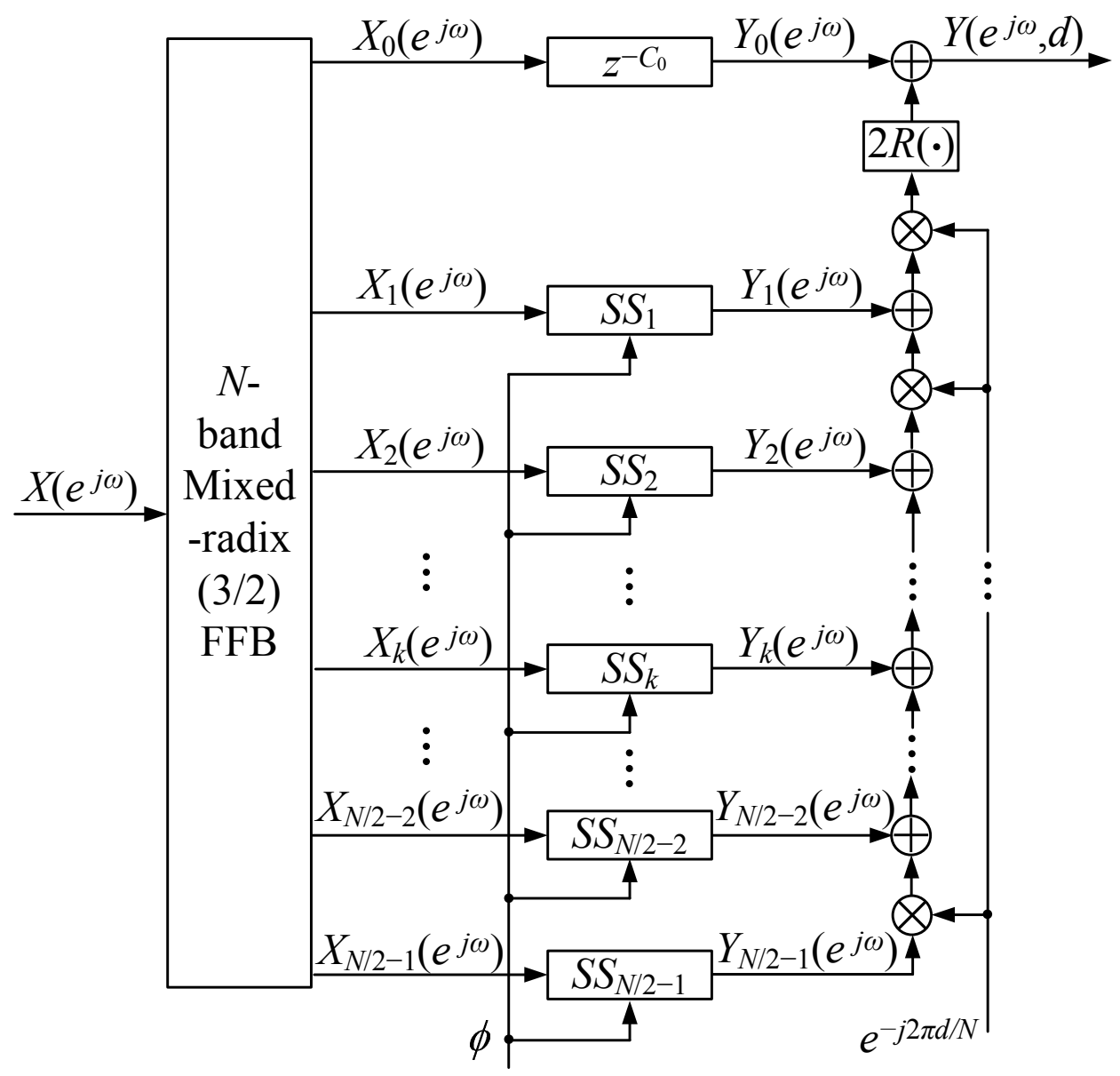

Figure 6.4. Block diagram of the synthesis of the VBFD filter with a configurable shaping structure.

Therefore, in the proposed technique, each of these bands is cascaded with a configurable shaping structure $S S_{k}$ for band $k$, as shown in Figure 6.4. The detailed shaping structure $S S_{k}$ is shown in Figure 6.5. For a particular $\phi$, let $H_{k}\left(e^{j \omega}\right)$ for $k=\bar{k}=$ $\left\lceil\frac{N(\phi+\gamma)}{2 \pi}\right\rceil$ be the last retained band. The switches $S_{\bar{k}, 1}$ and $S_{\bar{k}, 2}$ of the band $\bar{k}$ of the shaping structure are configured to port 2. Thus, the output of $H_{\bar{k}}\left(e^{j \omega}\right)$ is shaped by the shaping filter $F_{\bar{k}}\left(e^{j \omega}\right)$. The cascaded shaping structure of bands $H_{k}\left(e^{j \omega}\right)$ for $1 \leq k<\bar{k}$ are configured such that $S_{k, 1}$ and $S_{k, 2}$ are connected to port 1, and thus the outputs of these bands are delayed and aligned with that of band $\bar{k}$. For the remaining bands, i.e., band $k$ for $k>\bar{k}$, switch $S_{k, 2}$ is connected to port 3 and the signals of these bands are discarded. The structures in Figures 6.4 and 6.5 show that, at any moment with a particular $\phi$, only one shaping filter is in use. 


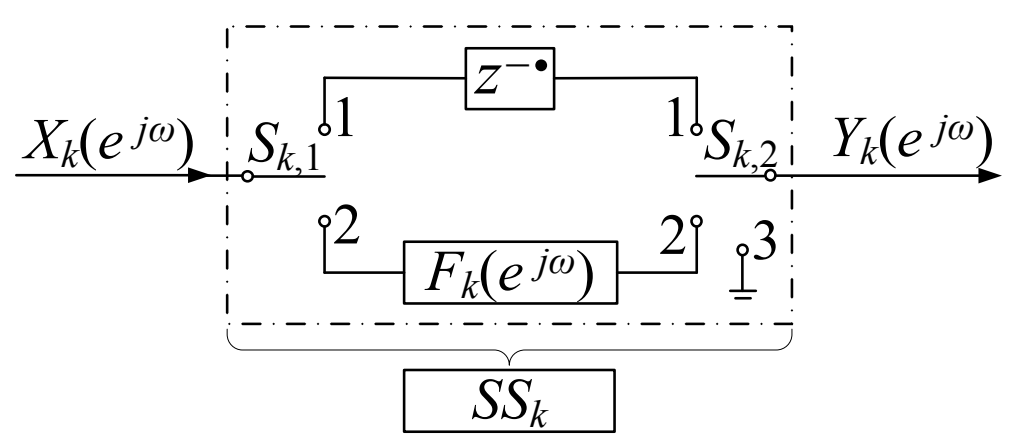

Figure 6.5. The configurable shaping structure to be cascaded to each band.

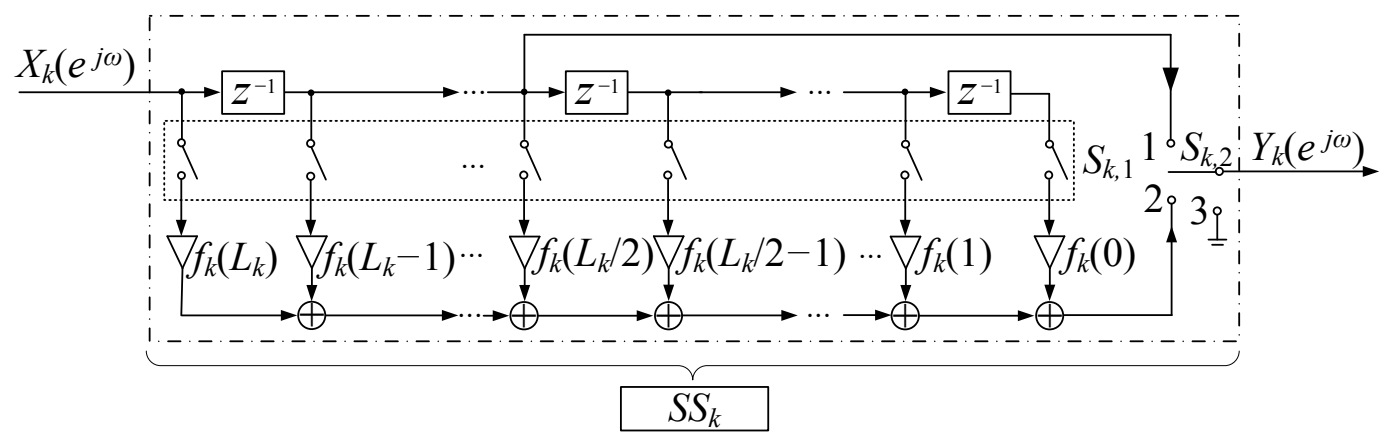

Figure 6.6. The implementation of the delay and shaping filter of a shaping structure in Figure 6.4.

To share the delays in the delay chain with that in $F_{k}\left(e^{j \omega}\right)$ within each shaping filter structure, the shaping filter $F_{k}\left(e^{j \omega}\right)$ is implemented in the direct form with embedded switches, as shown in Figure 6.6, where $f_{k}(l)$ for $l=0, \ldots, L_{k}$ is the impulse response of $F_{k}\left(e^{j \omega}\right)$ with even order $L_{k}$. For a varying $\phi$, the switches in Figure 6.6 for band $k$ are configured as that listed in Table 6.1.

The shaping structure proposed in Figure 6.6 also prevents the transient issue when a shaping filter $F_{k}\left(e^{j \omega}\right)$ gets connected or disconnected for the varying $\phi$, because the signals in each band are delayed properly.

\subsubsection{Sharing of the Shaping Filters}

The above subsection shows that the transition width of the VBFD filter can be reduced when each FFB band is cascaded with a configurable shaping structure. However, if the shaping filters in the shaping structures are implemented independently for each band, the implementation complexity may be high although the computational complexity is 
Table 6.1

SWITCH STATES FOR DELAY AND SHAPING FILTER BLOCK $k$ AND $\bar{k}=\left[\frac{N(\phi+\gamma)}{2 \pi}\right]$, WHERE $\phi$ IS THE PASSBAND EDGE, FOR THE SHAPING STRUCTURE IN FIGURE 6.6.

\begin{tabular}{c|c|c|c}
\hline \hline Switches & $k=\bar{k}$ & $k<\bar{k}$ & $k>\bar{k}$ \\
\hline$S_{k, 1}$ & on & off & off \\
\hline$S_{k, 2}$ & to port 2 & to port 1 & to port 3 \\
\hline \hline
\end{tabular}

controlled (because in every moment only one shaping filter is in use). In this subsection, an analysis shows that the shaping filters can be shared among different bands, and in total, at most three shaping filters are necessary to be implemented.

As shown in Figure 6.3, the function of the shaping filter $F_{k}\left(e^{j \omega}\right)$ is to mask out the frequency components of band $k$ from $\omega_{k}+\gamma$ onwards, and meanwhile keep the frequency components up to $\omega_{k}-\gamma$. Since the signal of band $k$ is a bandpass signal whose energy concentrates in the range of $\left[\omega_{k-1}-\gamma, \omega_{k+1}+\gamma\right]$, a bandpass filter with passband $\left[\omega_{k-1}-\right.$ $\left.\gamma, \omega_{k}+\gamma\right]$ can produce the necessary signal. On the other hand, the upper transition band of the bandpass filter is restricted to $\left[\omega_{k}-\gamma, \omega_{k}+\gamma\right]$ in order to accommodate the narrow $\Delta \omega$ given in (6.5). To have symmetric upper and lower transition bands for the bandpass filter, the lower transition band is given by $\left[\omega_{k-1}-3 \gamma, \omega_{k-1}-\gamma\right]$, as shown in Figure 6.7. It is noted that this bandpass shaping filter, denoted as $B_{3 i+k}\left(e^{j \omega}\right)$, can also be used for band $k+3 i$, if it contains a passband $\left[\omega_{k-1+3 i}-\gamma, \omega_{k+3 i}+\gamma\right]$, with the transition bands $\left[\omega_{k-1+3 i}-3 \gamma, \omega_{k-1+3 i}-\gamma\right]$ and $\left[\omega_{k+3 i}-\gamma, \omega_{k+3 i}+\gamma\right]$, for an integer $i$. Thus, $B_{3 i+k}\left(e^{j \omega}\right)$ is a multiple-band filter. To avoid that the energy of band $k$, both in the main lobe and the significant side lobes, leaks into the adjacent passband of $B_{3 i+k}\left(e^{j \omega}\right)$, i.e., band $k+3$, it is required that

$$
\omega_{k+2}-3 \gamma \geq \omega_{k+1}+\gamma
$$

Hence the side lobe width $\gamma$ should satisfy

$$
\gamma \leq \frac{\pi}{2 N}
$$




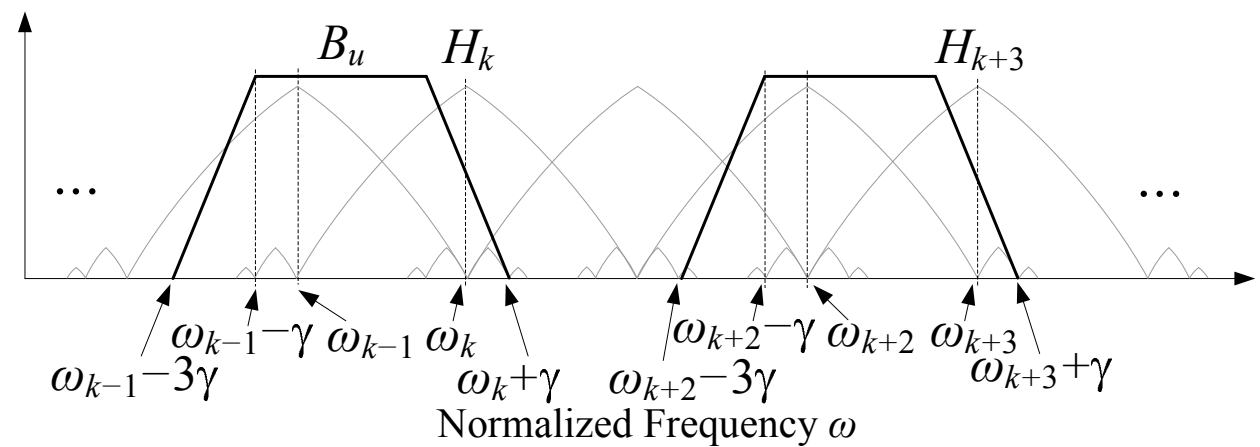

Figure 6.7. Frequency responses of bandpass shaping filters for bands $k$ and $k+3$.

Based on the above analysis, 3 such bandpass filters $B_{3 i+u}\left(e^{j \omega}\right)$, for an integer $i$ and $u=0,1,2$, are required to take care of the 3 sets of bands $3 i+u$. The three filters can be constructed from a prototype filter with a frequency compression factor $\frac{N}{3}$ and proper shifts. To reduce the computational complexity, the prototype filter is constrained to be a half band filter. This requires the passband width equals to the stopband width in Figure 6.7, i.e.,

$$
\omega_{k}-\gamma-\left(\omega_{k-1}-\gamma\right)=\omega_{k+2}-3 \gamma-\omega_{k}-\gamma
$$

Thus, it can be obtained that

$$
\gamma=\frac{\pi}{2 N}
$$

The prototype half band filter, denoted as $B\left(e^{j \omega}\right)$, is shown in Figure 6.8(a), with passband and stopband edges at $\frac{\pi}{3}$ and $\frac{2 \pi}{3}$, respectively. By frequency compression and shifting, the 3 shaping filters, denoted as $B_{u}\left(e^{j \omega}\right)$ for $u=0,1,2$, are given by

$$
B_{u}\left(e^{j \omega}\right)=B\left(e^{j \frac{N}{3}\left(\omega-\frac{4 u-3}{2 N} \pi\right)}\right)
$$

Figures 6.8(b)-(d) show the 3 bandpass filters $B_{u}\left(e^{j \omega}\right)$. Every band in each bandpass filter takes care of one set of FFB bands, if one of the sets of bands is to be shaped. Thus, these 3 filters can be shared among all the FFB outputs. The VBFD structure in Figure 6.6 is revised to Figure 6.9 when this sharing is employed, where $Q$ denotes the order of $B\left(e^{j \omega}\right)$. Figure 6.9(a) shows that each output of the FFB is connected to a delay chain. The tap delayed signals are either connected to one of the three bandpass filters 
(a)

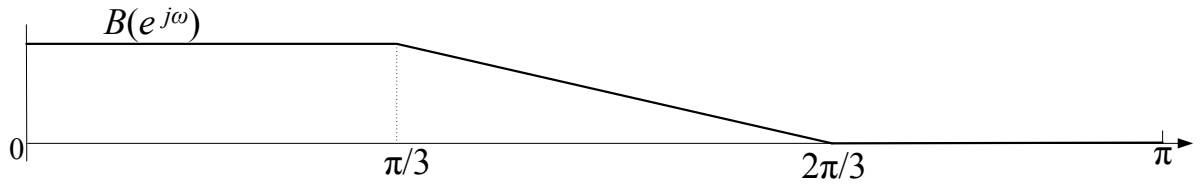

(b)

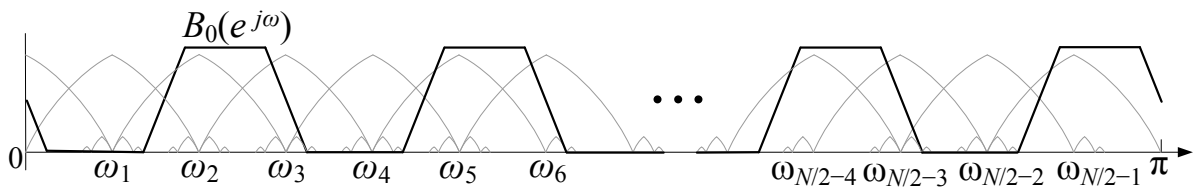

(c)

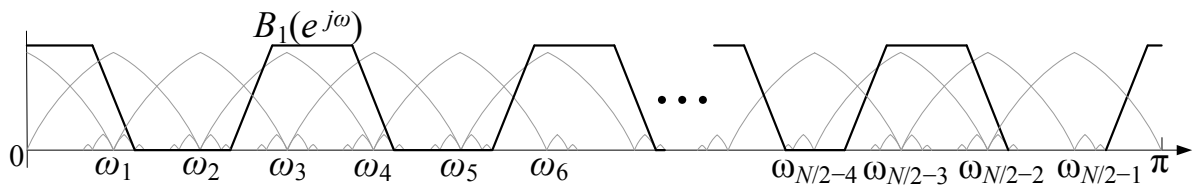

(d)

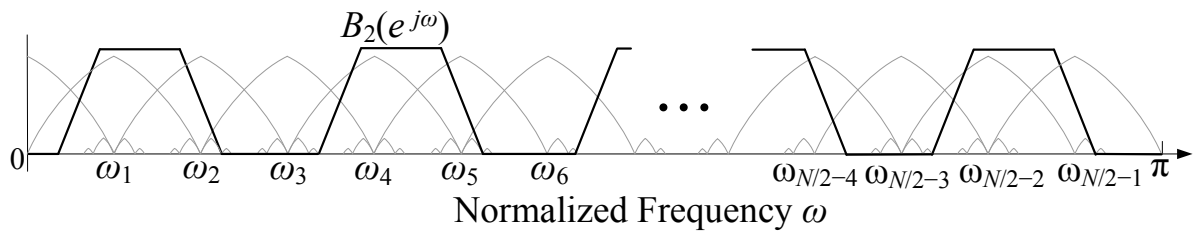

Figure 6.8. The frequency responses of (a) the prototype filter (b) $B_{0}\left(e^{j \omega}\right)$ (c) $B_{1}\left(e^{j \omega}\right)$ (d) $B_{2}\left(e^{j \omega}\right)$.

by turning on $S_{k, 1}$ and switching $S_{k, 2}$ to port 2, if the band $k$ is the last retained band $\bar{k}$, or directly sent to the right adder chain by turning off $S_{k, 1}$ and switching $S_{k, 2}$ to port 1 , if $k<\bar{k}$, or discarded by switching $S_{k, 2}$ to port 3, if $k>\bar{k}$. Figures 6.9(b)-(d) show the coefficient multipliers of the 3 shaping filters, where $b_{u}(n)$ for $n=0, \ldots, Q$ are the impulse responses of $B_{u}\left(e^{j \omega}\right)$. The suspending terminals in Figure 6.9(a) for band $k$ are connected to the filter $B_{u}\left(e^{j \omega}\right)$ in Figures 6.9(b)-(d) for

$$
u=\bmod (k, 3)
$$

as labeled in Figures 6.9. The ports Out_u, $\left(L_{0}, u\right),\left(L_{1}, u\right), \ldots,\left(L_{Q}, u\right)$ of the delay lines in each channel and those of the shaping filters in Figures 6.9(b)-(d) are connected for $u=0,1,2$, respectively.

\subsection{Complexity Analysis}

In this section, the computational and implementation complexities of the proposed technique without and with band shaping filters are analyzed. The numbers of multiplications 


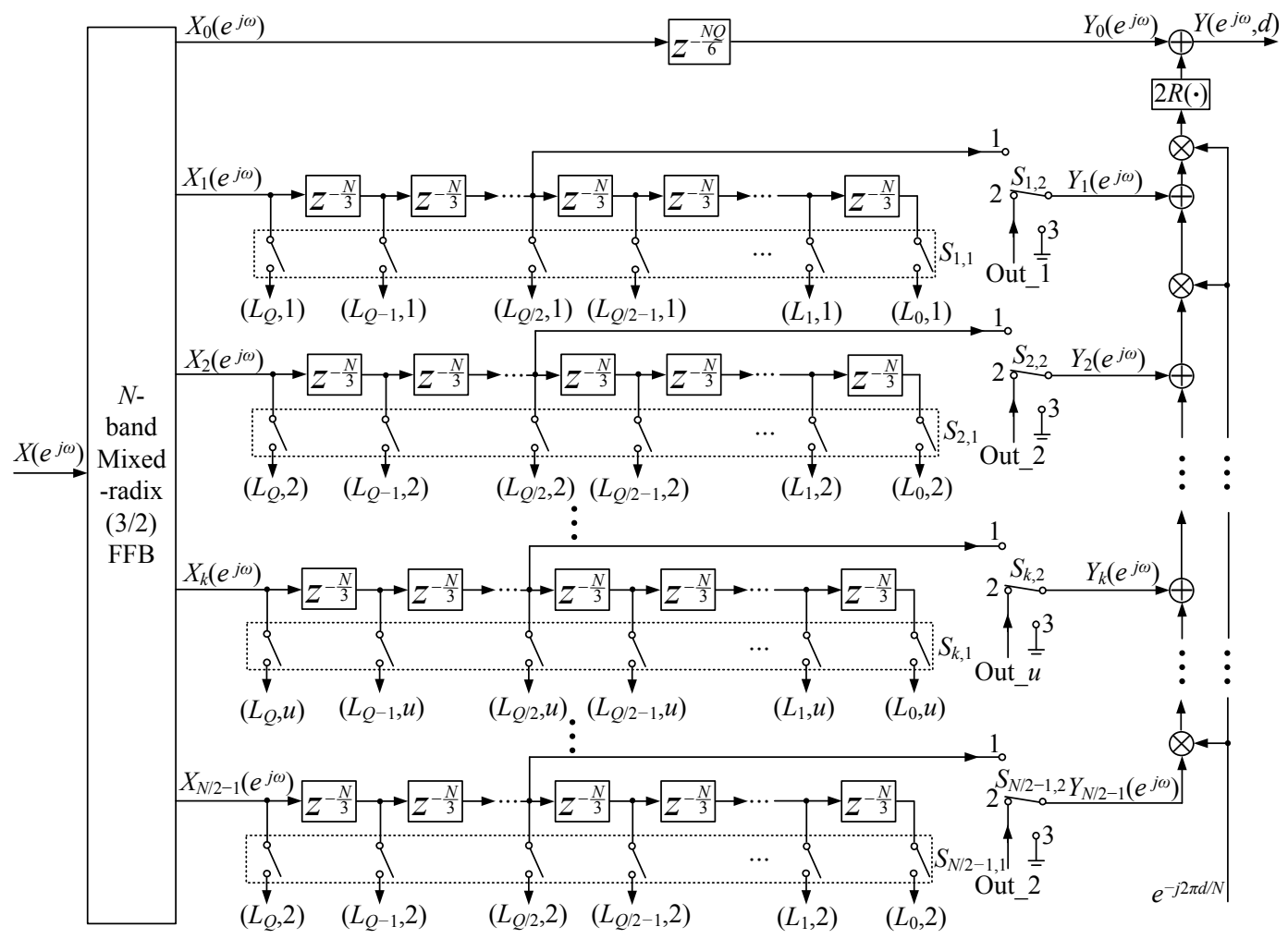

(a)

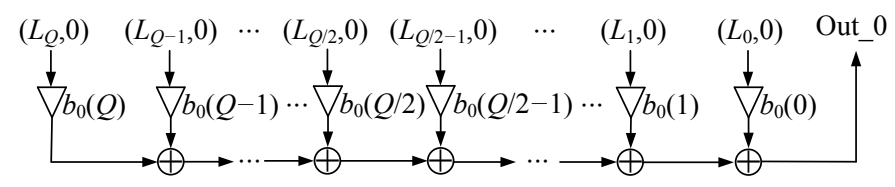

(b)

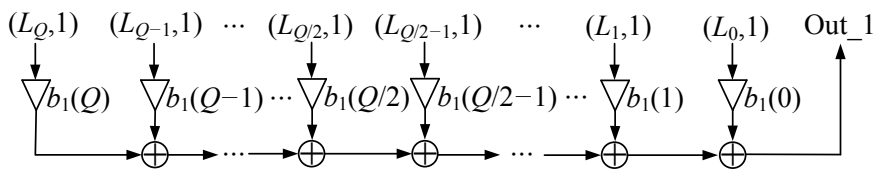

(c)

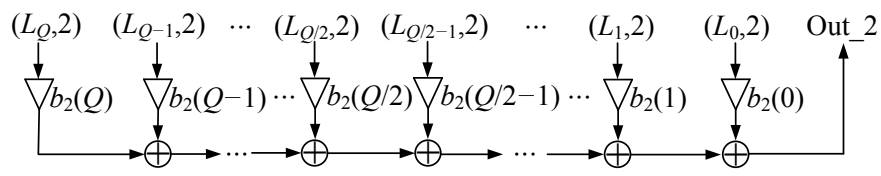

(d)

Figure 6.9. The structure of (a) an $R$-stage $N$-band mixed-radix FFB with the technique of shaping filter sharing and (b)(c)(d) the multiplier blocks of the 3 shaping filters, respectively. 
per output sample are investigated in the computational complexity and the numbers of multipliers and delay units are calculated in the implementation complexity.

\subsubsection{Computational Complexity}

As given in (6.6), $N_{H}$ denotes the last retained FFB band that may served as the passband of the VBFD filter. For the technique without shaping filters, the number of real multiplications required for each sample out, denoted as $C_{m u l, n b s}$, has already been given in (5.12) as

$$
\begin{aligned}
C_{\text {mul }, \text { nbs }} & =C_{F F B}+C_{\text {phase }} \\
& =\left(\frac{K_{0}+2}{2}\right) \times 3-1+\left(\frac{K_{1}+2}{4}\right) \times 4-1 \\
& +\sum_{r=2}^{R-1}\left(\frac{K_{r}+2}{4}\left(9 \times 2^{r-2}\right)-3 \times 2^{r-2}\right)+3 N_{H}+9 \\
& =\left(\frac{K_{0}+2}{2}\right) \times 3-1+\left(\frac{K_{1}+2}{4}\right) \times 4-1 \\
& +\sum_{r=2}^{R-1}\left(3 \times \frac{K_{r}+2}{4}-1\right)\left(3 \times 2^{r-2}\right)+3 N_{H}+9 .
\end{aligned}
$$

where $3 N_{H}+8$ are general multiplications to multiply the phase shift of each retained band signal and the others are constant multiplications. In the notation, $C_{m u l, n b s}$, "nbs" stands for "no band shaping".

When the shaping filters are employed, extra multiplications are introduced. Denote the number of multiplications incurred for each output sample due to the shaping filters as $C_{\text {shaping. }}$ It is noted that the shaping filters $B_{u}\left(e^{j \omega}\right)$ for $u=1,2$, constructed from $B\left(e^{j \omega}\right)$, are complex-valued coefficients, whereas $B_{0}\left(e^{j \omega}\right)$ is a pure imaginary coefficient filter,

and the signals from band $1, \ldots, \frac{N}{2}-1$ are complex. Hence, in the worst case, i.e., either $B_{1}\left(e^{j \omega}\right)$ or $B_{2}\left(e^{j \omega}\right)$ is used for each sample output, $\frac{Q+2}{4}$ times of complex multiplications are required. Since the multiplication of two complex numbers can be realized using 
three real multiplications [134], the value of $C_{\text {mul,shaping }}$ hence is given by

$$
C_{\text {mul,shaping }}=\frac{3}{4}(Q+2)-1 \text {. }
$$

Therefore, the number of multiplications for each sample output, $C_{m u l, b s}$, is given as,

$$
\begin{aligned}
C_{\text {mul }, \text { ss }} & =C_{\text {mul,shaping }}+C_{\text {mul }, n b s} \\
& =\frac{3}{4}(Q+2)-1+\left(\frac{K_{0}+2}{2}\right) \times 3-1+\left(\frac{K_{1}+2}{4}\right) \times 4-1 \\
& +\sum_{r=2}^{R-1}\left(3 \times \frac{K_{r}+2}{4}-1\right)\left(3 \times 2^{r-2}\right)+3 N_{H}+9,
\end{aligned}
$$

where $b s$ stands for "with band shaping".

\subsubsection{Implementation Complexity}

The multipliers implemented for the FFB and phase shifting without shaping filters, denoted as $I_{m u l, n b s}$, is the same as the number of multiplications to produce an output sample, i.e.,

$$
I_{m u l, n b s}=C_{m u l, n b s}
$$

The delay units in this case, are all used for the construction of the FFB. As shown

in Figure 5.3, if the filters are implemented in direct form, the branches $G_{0,0}$ and $G_{0,1}$ of stage 0 have the same signal input and hence the delay line can be shared; in the remaining stages, each filter pair $G_{r, q}$ can share the delay chain. Depending on the complex-valued or real-valued coefficients and signals as described in Section 5.1.3, the number of overall delay units is given as

$$
\begin{aligned}
I_{\text {del }, n b s} & =2^{R-1} K_{0}+3 \times 2^{R-2} K_{1}+\sum_{r=2}^{R-1} 2^{R-1-r}\left(1+1+2 \times\left(3 \times 2^{r-2}-1\right)\right) K_{r} \\
& =2^{R-1} K_{0}+3 \times 2^{R-2} \sum_{r=1}^{R-1} K_{r}
\end{aligned}
$$

For the proposed band shaping technique, the operation of band shaping incurs extra implementation complexity. Three shaping filters are implemented, where the coefficients of $B_{0}\left(e^{j \omega}\right)$ are pure imaginary values and the other 2 are complex values. The 
number of real multipliers required for band shaping is thus given as

$$
I_{\text {mul,shaping }}=2 \times \frac{Q+2}{4}+2 \times\left(3 \times \frac{Q+2}{4}-1\right)=2 Q+2
$$

Hence, the total number of real multipliers for the proposed technique with band shaping is given as

$$
I_{m u l, b s}=I_{m u l, n b s}+I_{m u l, s h a p i n g}
$$

As for delay units, each band for band $1, \ldots, N_{H}$ requires $2 \times \frac{N Q}{3}$ delays, whereas band 0 requires $\frac{N Q}{6}$ delays. Hence the number of delays for the band shaping is given by

$$
I_{\text {del,shaping }}=N_{H} \times \frac{2 N Q}{3}+\frac{N Q}{6}=\left(4 N_{H}+1\right) \frac{N Q}{6} .
$$

Therefore, the total number of delay units implemented for the proposed technique with band shaping is given by

$$
I_{d e l, b s}=I_{d e l, \text { shaping }}+I_{d e l, n b s}=\left(4 N_{L}+1\right) \frac{N Q}{6}+2^{R-1} K_{0}+3 \times 2^{R-2} \sum_{r=1}^{R-1} K_{r} .
$$

\subsubsection{Switch Complexity}

Both Proposed ${ }_{1}$ and Proposed ${ }_{2}$ approaches introduce switches for band selection.

For Proposed ${ }_{1}$, switches are used to select which bands to compose the overall output. The number of total switches is given as

$$
N_{s, n b s}=N_{H}-N_{L}+1
$$

where $N_{H}$ and $N_{L}$ are given in (6.8) and (6.7), respectively.

For Proposed ${ }_{2}$, besides selecting which bands to compose the overall output, switches are also used to select which band is filtered by the shaping filter. The number of total switches is given as

$$
N_{s, b s}=(Q+1)\left(N_{H}-N_{L}+1\right)+\left(N_{H}-N_{L}+1\right)=(Q+2)\left(N_{H}-N_{L}+1\right)
$$


Switches can be simply realized by transmission gates, each containing 2 transistors. To control the switches, comparators are required. According to Figure 6.2 and Table 6.1, 3 and 6 comparators are required respectively for Proposed Pa $_{1}$ Proposed ${ }_{2}$. Each comparator can be realized by either an XOR and an AND gate (for equal comparison) or an subtractor (for larger or smaller comparison). Hence, the implementation complexity incurred due to the switches is very low.

In addition, the transmission gates and the comparators switch only when the desired bandedge of the filter varies. Hence the power consumption incurred due to the switches is negligible.

From Figure 6.2(b)(c)(d) and Table 6.1, it can be found that the switches in both structures are combinational logic circuits, which of each is composed of an XOR (exclusive or) gate and a comparator. In digital circuits, an XOR gate can be simply implemented using 4 NAND gates and a comparator can be replaced by a transmission gate. Hence the control complexity due to switching activity is negligible.

\subsection{Design Parameters}

In this section, the determination of the stage number $\mathrm{R}$ and the adoption of the shaping filters are discussed.

\subsubsection{Determination of the Number of the FFB Stages}

Similar to the design of VFD filters, two filter specifications determine the number of stages of the FFB for the design of VBFD filters. The first specification is the tran-

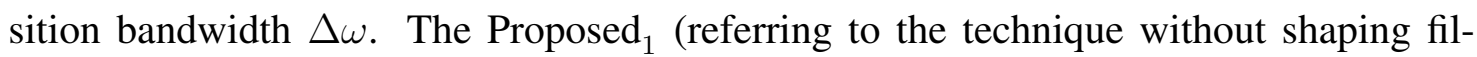
ters) requires the number of bands of the FFB $N=N_{1} \geq \frac{5 \pi}{\Delta \omega}$ according to (33) when $\gamma=\frac{\pi}{2 N}$. With shaping filters, $N$ is relaxed to $N=N_{2} \geq \frac{3 \pi}{\Delta \omega}$ according to (36) for the Proposed $_{2}$ (referring to the technique with shaping filters). The second specification is the magnitude and phase error requirements. In the working bandedge variation range 
$\left[\max \left(0, \frac{5 \pi}{2 N}-\Delta \omega\right), \pi-\frac{5 \pi}{2 N}\right]$, the maximum magnitude and phase errors decrease when $R$ and/or $\Delta \omega$ increase, as shown in Figure 6.10. Therefore, the selection of $R$ must satisfy the condition that the corresponding magnitude and phase errors are smaller than $\delta_{m}$ and $\delta_{p}$, respectively.

\subsubsection{Adoption of the Shaping Filters}

Once the numbers of the FFB stages for the Proposed ${ }_{1}\left(N_{1}\right)$ and the $\operatorname{Proposed}_{2}\left(N_{2}\right)$ are determined according to the above considerations, whether the Proposed ${ }_{2}$, i.e., the technique with the shaping filters, can be used to further reduce the complexity is determined as follows: if $N_{2}$ is smaller than $N_{1}$, the Proposed ${ }_{2}$ is used since the Proposed 2 is beneficial only if the number of the FFB stages can be reduced, compared with the Proposed $_{1}$.

\subsubsection{Summary of Design Steps}

The steps for the design of VBFD filters are summarized as follows:

1. For the given specifications of $\Delta \omega, \delta_{m}$ and $\delta_{p}$, the numbers of the FFB stages for the Proposed ${ }_{1}$ and the Proposed ${ }_{2}$ are determined according to Section 6.4.1;

2. Determine if the Proposed ${ }_{1}$ or the Proposed $_{2}$ is used according to Section 6.4.2;

3. The half band prototype filters $G_{r}\left(e^{j \omega}\right)$ for $r=1,2 \ldots, R-1$ are optimized in the same way at that for the VFD filters, described in the third paragraph of Section 5.3;

4. The order of the 0th stage prototype filter is determined also in the same way as that for the VFD filters, described in the last paragraph of Section 5.3. And $G_{0}\left(e^{j \omega}\right)$ is optimized by solving the problem formulated in (6.4);

5. The half band prototype shaping filter is designed according to the bandedges and ripple tolerances given in Section 6.2.2 if the introduction of shaping filters is beneficial. 


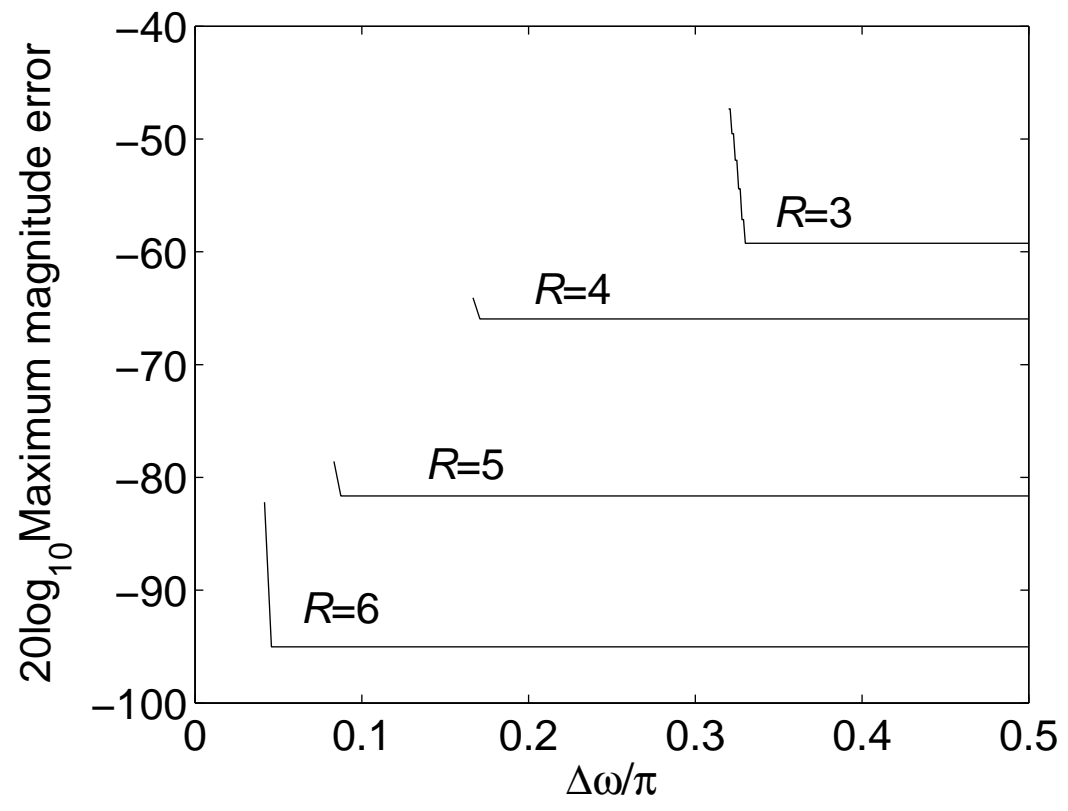

(a)

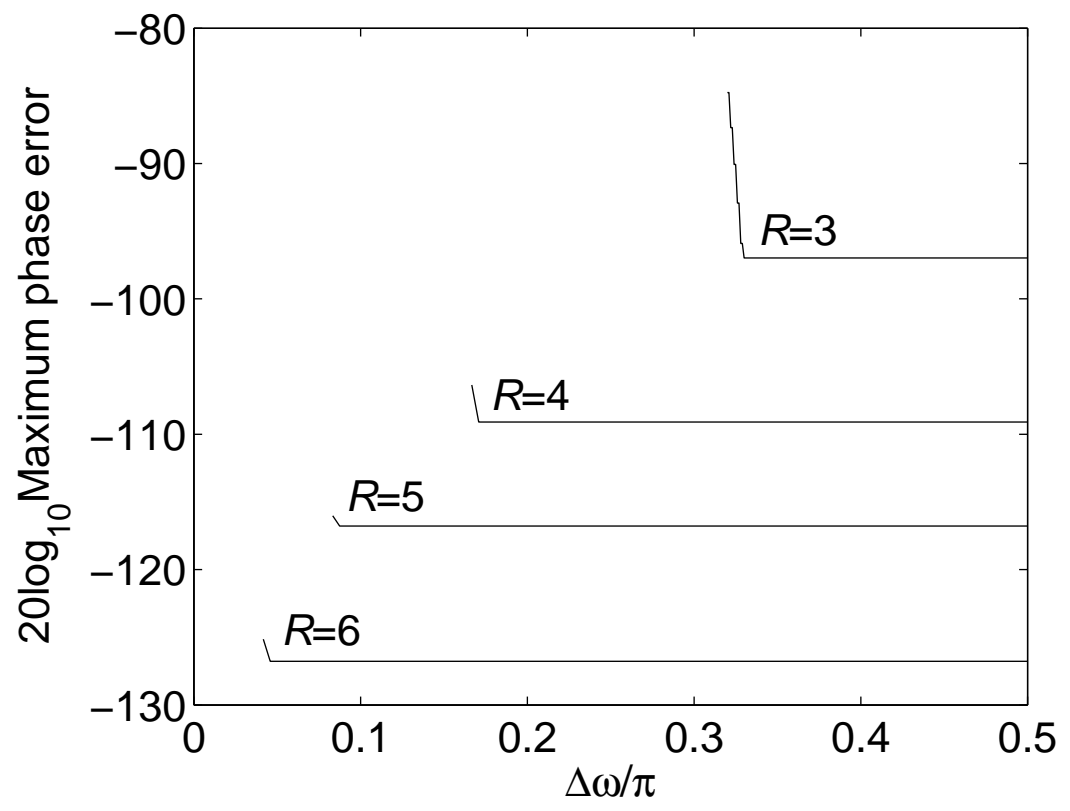

(b)

Figure 6.10. The maximum (a) magnitude and (b) phase errors vs. $\Delta \omega$ for $R=3,4,5$ and 6. 


\subsection{Design examples}

Three design examples are presented to illustrate the different scenarios of the proposed approaches for the design of the VBFD filters.

\subsubsection{Example A}

The example is taken from [91] and the specifications of the VBFD filter are given as follows:

$\phi \in[0.1 \pi, 0.42 \pi]$ (passband variation range);

$d \in[-0.5,0.5]$ (fractional delay variation range);

$\Delta \omega=0.24 \pi$ (transition band width);

$\delta_{m, p}=\delta_{m, s}=0.023$ (maximum magnitude error of the passband and the stopband);

$\delta_{\Phi}=0.003$ (maximum phase error of the passband).

To meet the specifications, the proposed technique requires a 4 -stage $(N=24)$ FFB and a 3-stage $(N=12)$ FFB for the Proposed 1 and the Proposed ${ }_{2}$, respectively. The orders of the prototype filters for the two FFBs are $K_{0}=8, K_{1}=10, K_{2}=6, K_{3}=6$ and $K_{0}=8, K_{1}=14$ and $K_{2}=6$, respectively, where in the case of the Proposed ${ }_{2}$, the order $Q$ of the prototype filter for band shaping is 14 .

Table 6.2 shows the computational and implementation complexities of the filters designed using the proposed techniques Proposed $\mathrm{d}_{1}$ and Proposed $_{2}$, and the comparisons with that of [91]. Compared with [91], the Proposed ${ }_{1}$ has significant savings in the arithmetic units and operations at the price of a moderate increase in the number of delay units to be implemented, while the saving of the Proposed $_{2}$ is more significant.

The maximum magnitude and phase errors vs. $d$ and $\phi$, of the filters obtained using

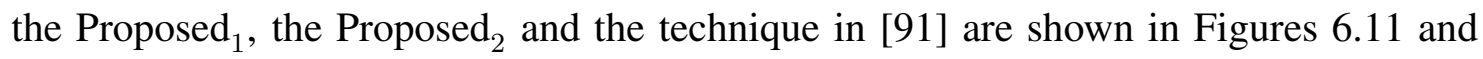
6.12 , respectively. The magnitude and phase errors for an arbitrary chosen passband 


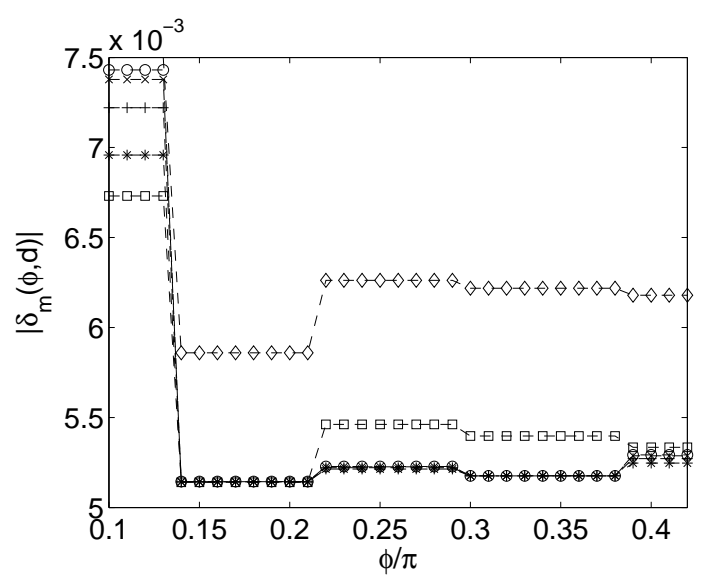

(a)

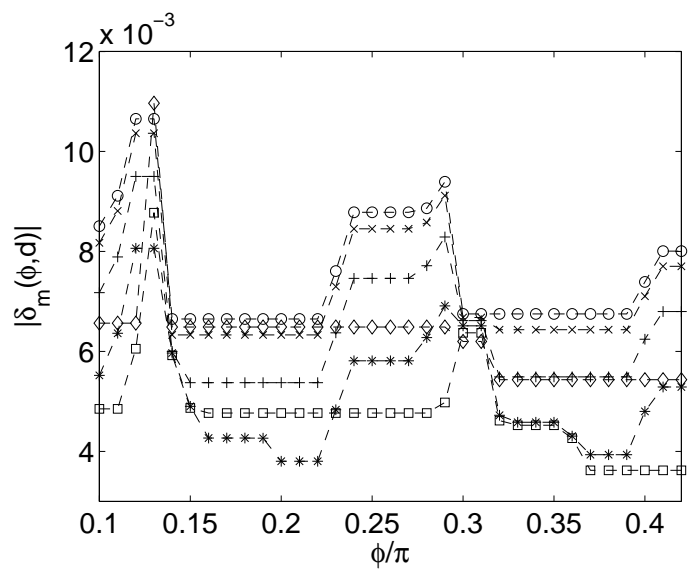

(b)

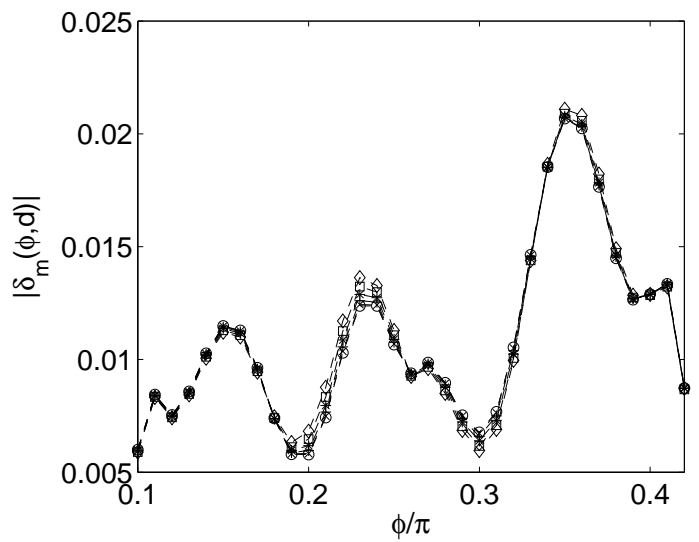

(c)

Figure 6.11. Example A: the maximum magnitude errors vs. $d$ and $\phi$, of the VBFD filters designed by (a) the $\operatorname{Proposed}_{1}$ (b) the $\operatorname{Proposed}_{2}$ (c) the technique in [91]. 


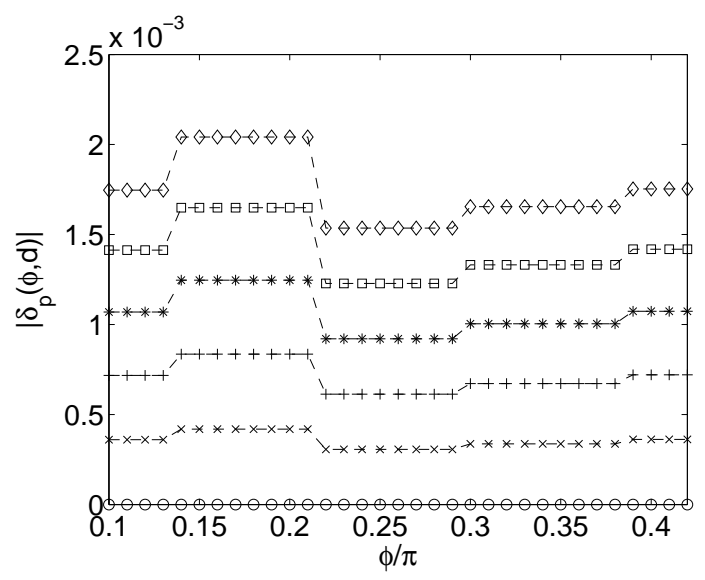

(a)

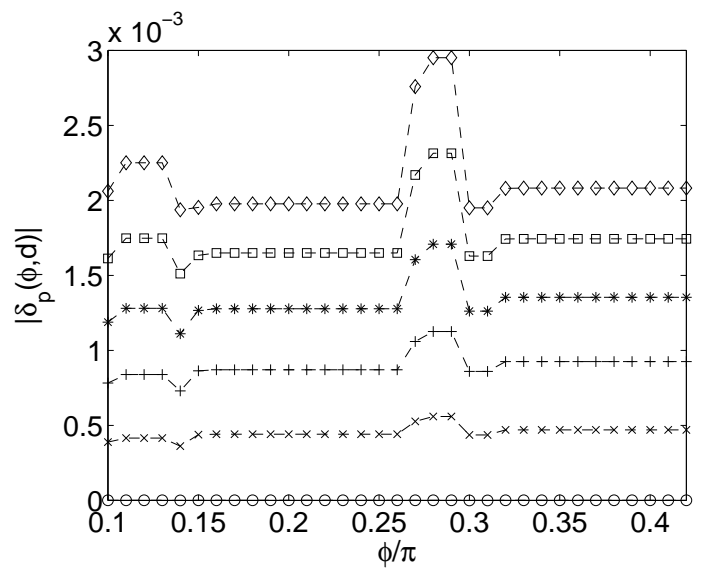

(b)

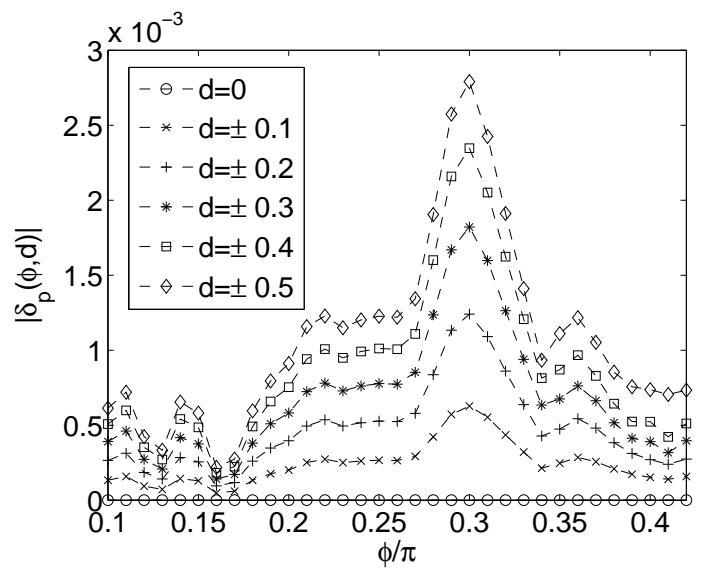

(c)

Figure 6.12. Example A: the maximum phase errors vs. $d$ and $\phi$, of the VBFD filters designed by (a) the Proposed $_{1}$ (b) the Proposed 2 (c) the technique in [91]. 


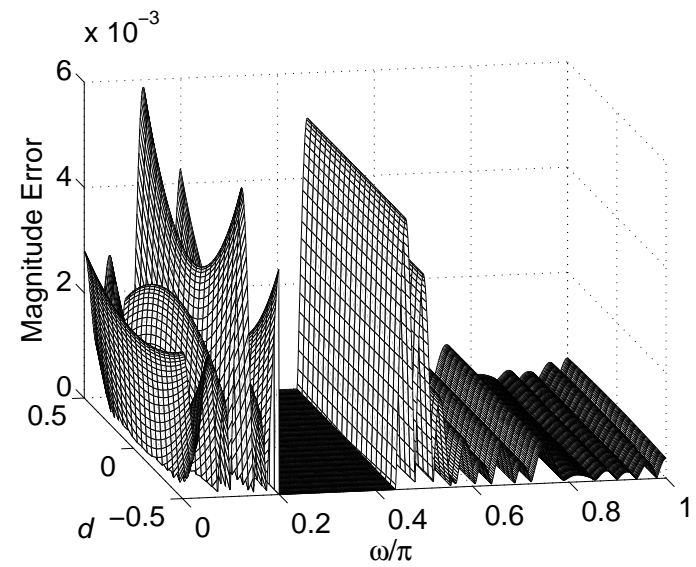

(a)

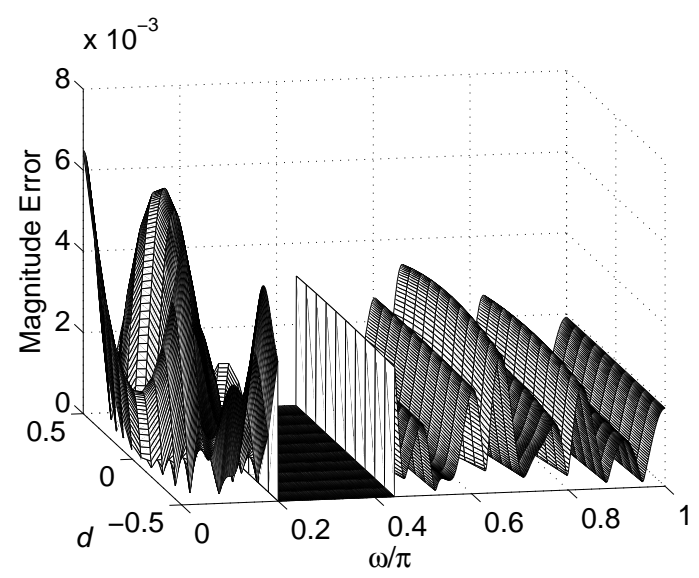

(c)

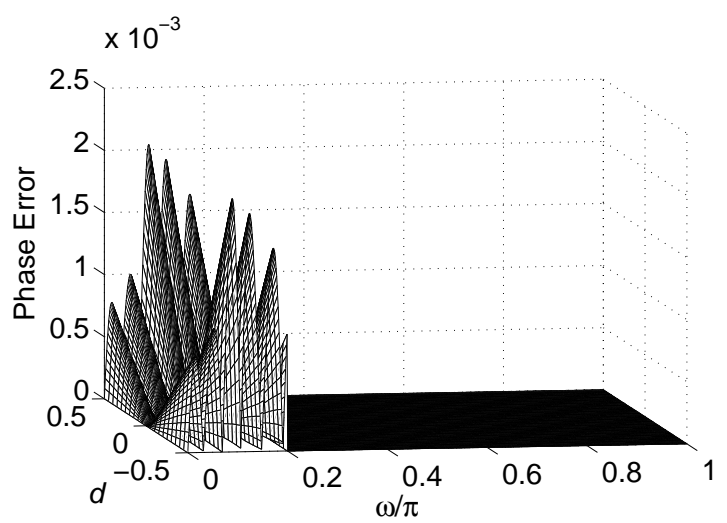

(b)

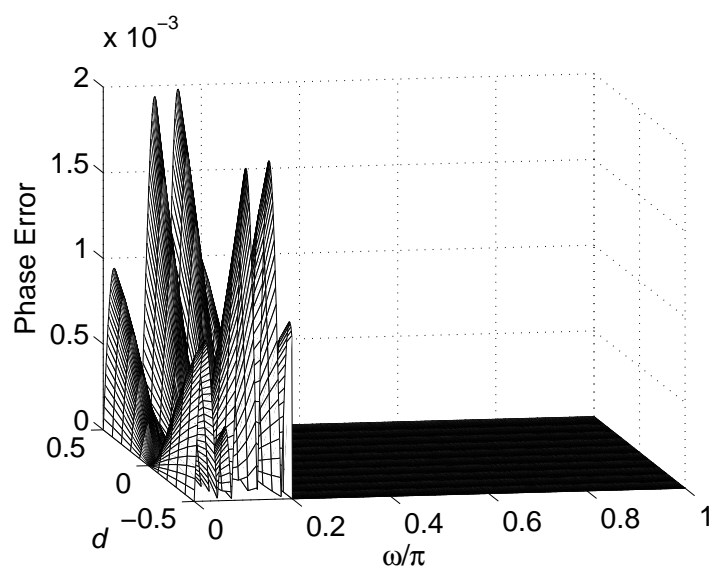

(d)

Figure 6.13. Example A: (a)(c)Magnitude errors and (b)(d) phase errors of the filters designed using the Proposed $\mathrm{P}_{1}$ and the Proposed ${ }_{2}$, respectively, for $\phi=0.2 \pi$. 


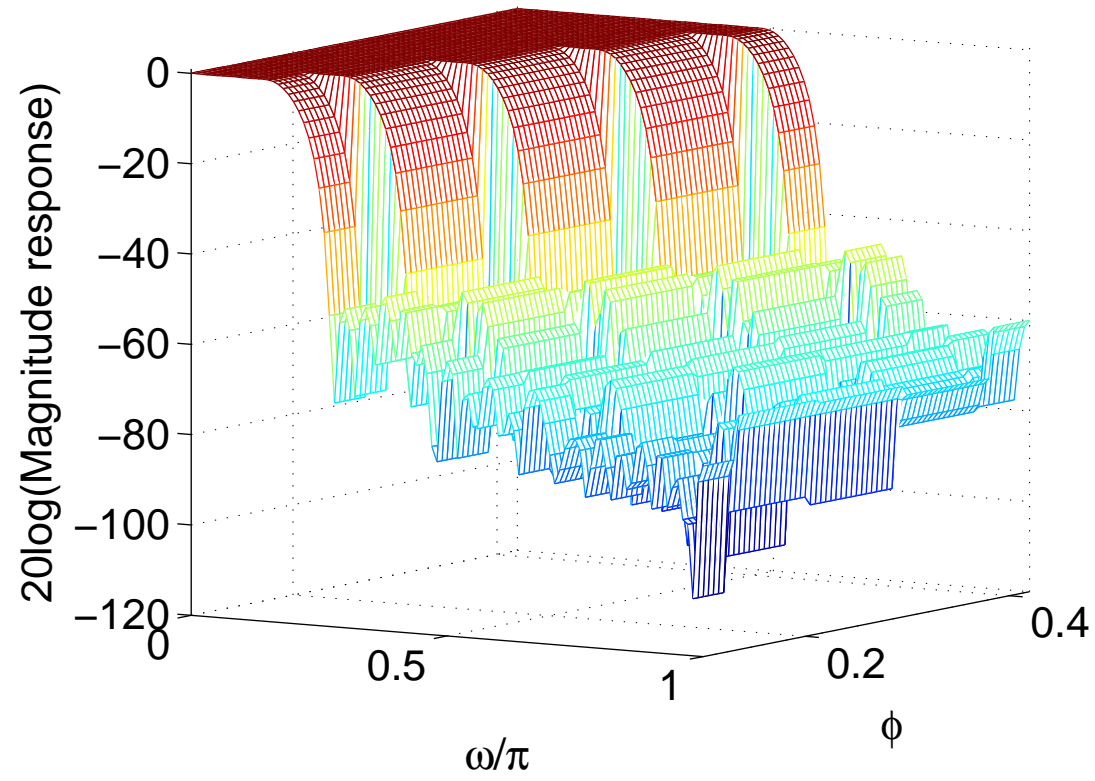

(a)

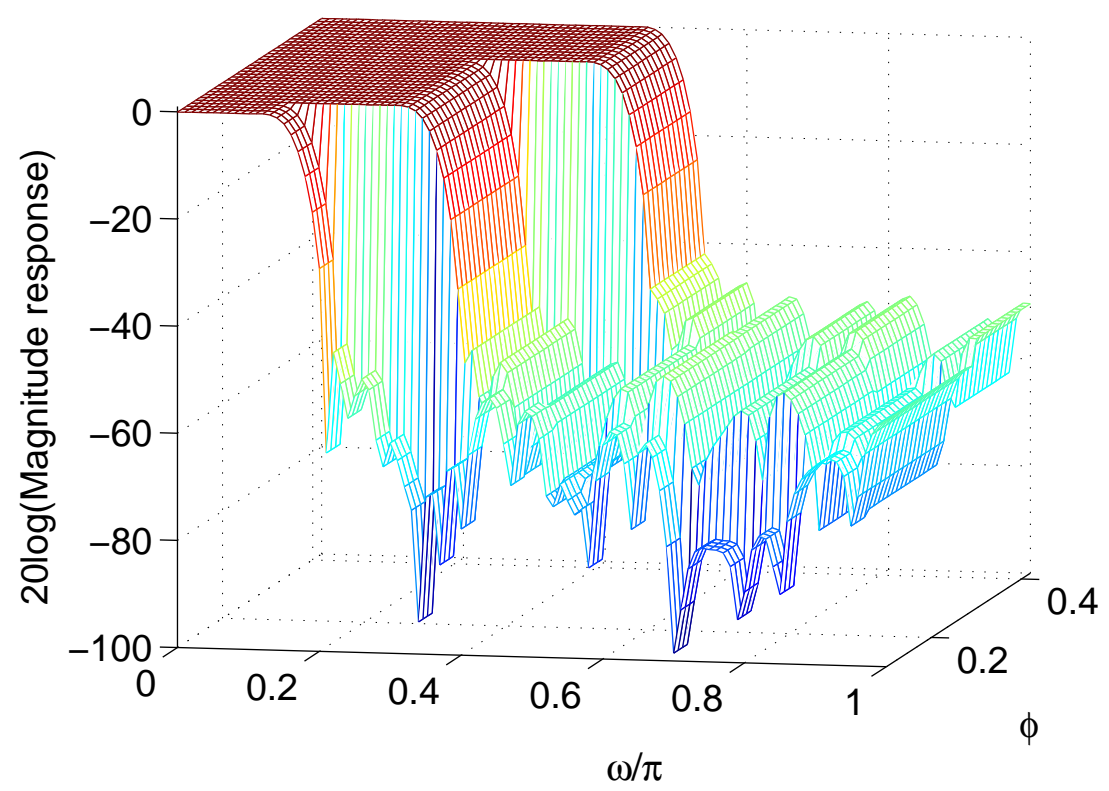

(b)

Figure 6.14. Example A: the magnitude responses of the VBFD filters with passband edge $\phi$ varying from $0.1 \pi$ to $0.42 \pi$ and an arbitrarily selected fractional delay $d=0.4$ using (a) the Proposed 1 and (b) the Proposed ${ }_{2}$, respectively. 
edge $0.2 \pi$ are shown in Figures 7.3. The magnitude responses of the VBFD filters using the Proposed ${ }_{1}$ and the Proposed 2 vs. $\phi$ for $d=0.4$ are shown in Figures 6.14 (a) and (b), respectively.

\subsubsection{Example B}

The example is taken from [92], where the passband variation range as well as the transition band is the same as that in Example A, but the specifications of the maximum magnitude and phase errors are given as:

$$
\begin{aligned}
& \delta_{m, p}=\delta_{m, s}=0.03 \\
& \delta_{\Phi}=0.0005 .
\end{aligned}
$$

To meet the specifications, the proposed technique requires a 4-stage $(N=24)$ FFB for the Proposed ${ }_{1}$, and the orders of the prototype filters are $K_{0}=10, K_{1}=18, K_{2}=10$ and $K_{3}=6$, respectively. In this example, no additional saving is achieved if shaping filters are employed.

The maximum magnitude and phase errors are shown in Figures 6.15(a) and (b), respectively.

The comparison of the computational and implementation complexity of the technique in [92] and the Proposed ${ }_{1}$ is listed in Table 6.3. From Table 6.3, it is seen that Proposed $_{1}$ significantly reduces the numbers of constant multiplications and additions at the cost of a larger group delay and more delay units, compared with the technique in [92].

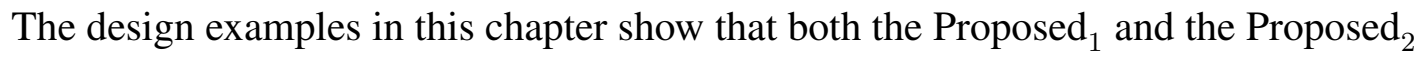
achieve significant savings in the arithmetic operation and units, compared with [92]. Although the hardware cost is saved, the proposed designs result in longer latencies and hence they are not preferred in low group delay applications. This technique offers tradeoffs between the arithmetic complexity and delay elements, and provides more options 


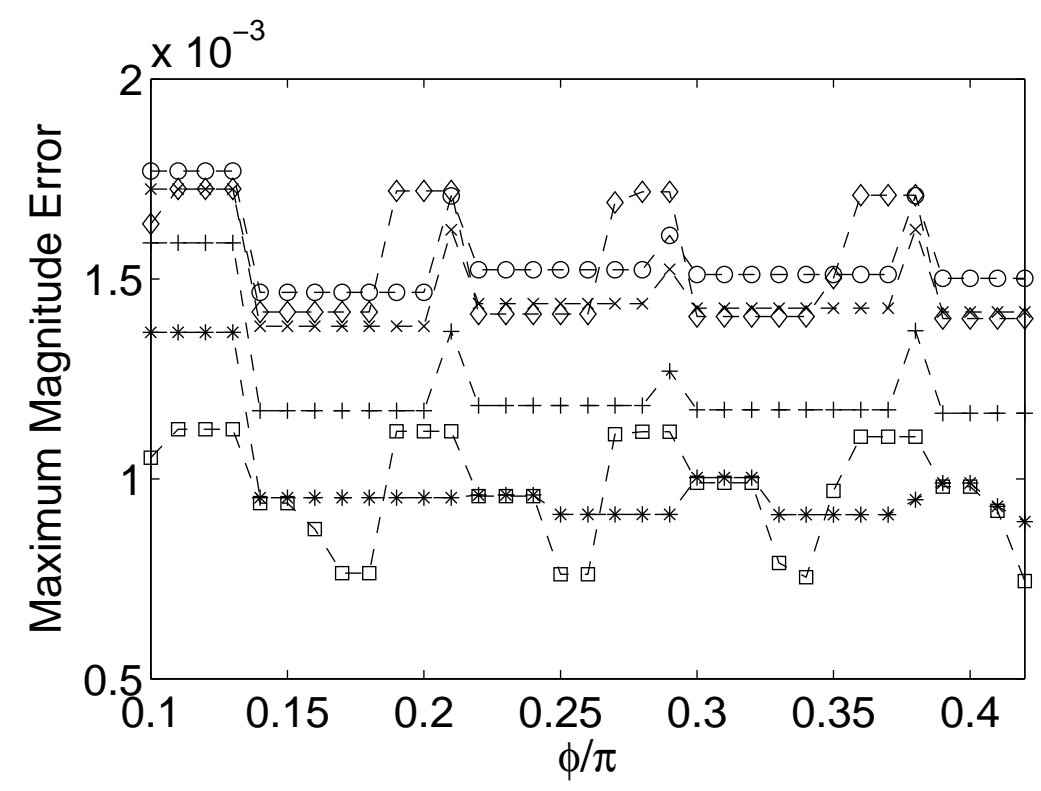

(a)

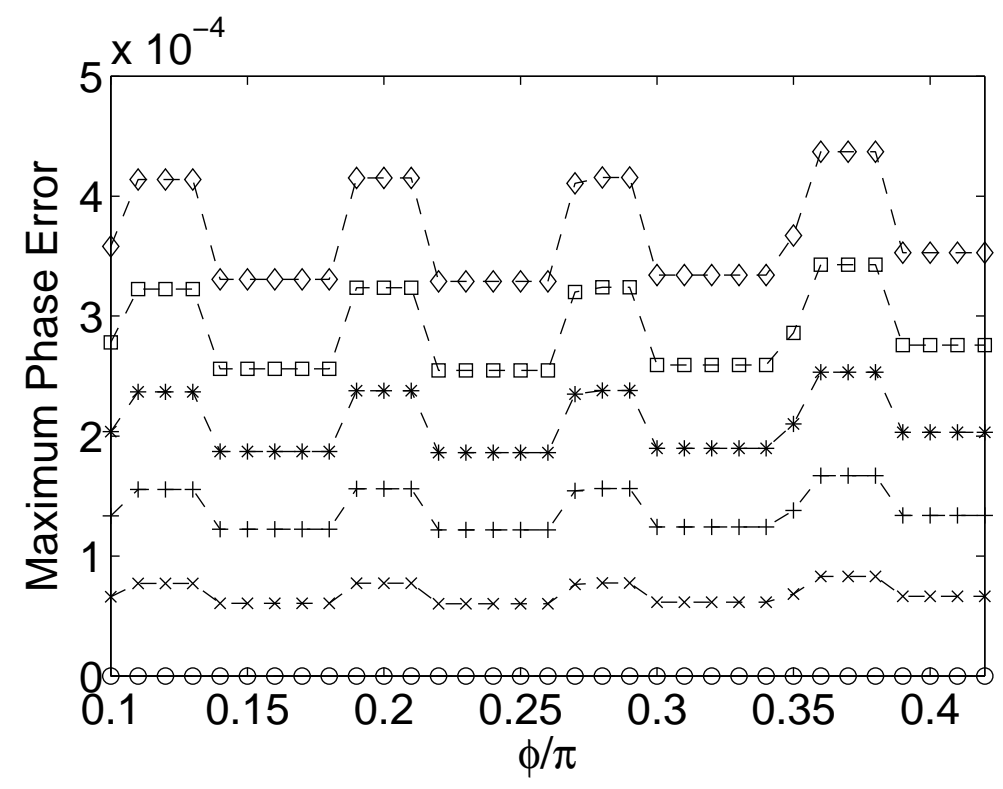

(b)

Figure 6.15. Example B: the maximum (a) magnitude errors and (b) phase errors vs. $d$ and $\phi$ of the VBFD filters designed by the Proposed . $_{\text {. }}$ 
in the implementation, and the preferred structure will be determined by the cost ratio between arithmetic and delay units which in turn depends on hardware architectures and platforms.

\subsubsection{Example C}

In this example, the specifications are more stringent, i.e., sharper transition bandwidth, smaller magnitude and phase error tolerances and wider passband variation range, as follows:

$$
\begin{aligned}
& \phi \in[0.06 \pi, 0.64 \pi] ; \\
& d \in[-0.5,0.5] ; \\
& \Delta \omega=0.15 \pi ; \\
& \delta_{m}=2 \times 10^{-3} ; \\
& \delta_{p}=8 \times 10^{-4} .
\end{aligned}
$$

A 5-stage $(N=48)$ FFB and a 4-stage $(N=24)$ FFB are required for the Proposed 1 and the Proposed ${ }_{2}$, respectively. The orders of the prototype filters for the two FFBs are $10,18,14,10,6$ and 14, 22, 10, 6, respectively. In the case of the Proposed ${ }_{2}$, the order of the prototype filter for band shaping is 14 .

The maximum magnitude and phase errors vs. $d$ and $\phi$ of the design examples are illustrated in Figures 6.16 and 6.17, respectively. The magnitude responses of the VBFD filters using the Proposed ${ }_{1}$ and the Proposed ${ }_{2}$ vs. $\phi$ for $d= \pm 0.3$ are shown in Figures 6.18(a) and (b), respectively.

While the three techniques all meet the design specification, Table 6.4 lists the computational and implementation complexity of each technique. When shaping filters are applied, the numbers of both multiplications (multipliers) and additions (adders) of the computational (implementation) complexity are significantly reduced, but at the price of significant increase in the implementation of delay units. 


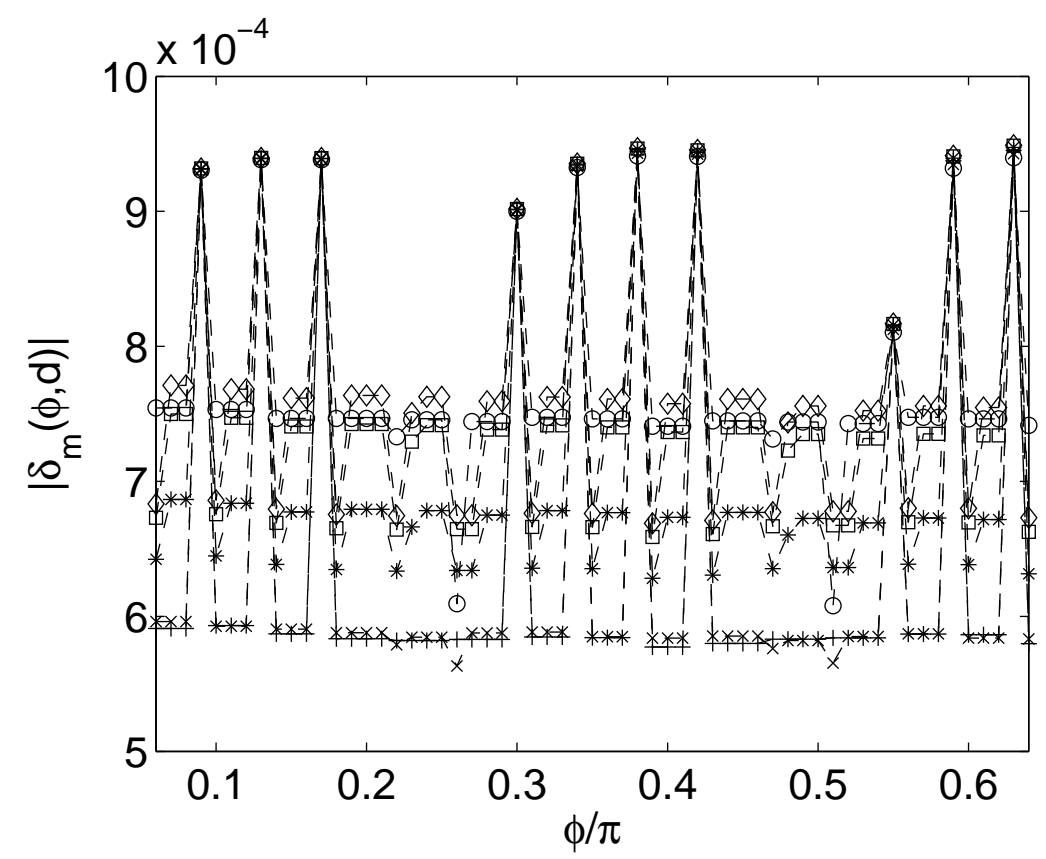

(a)

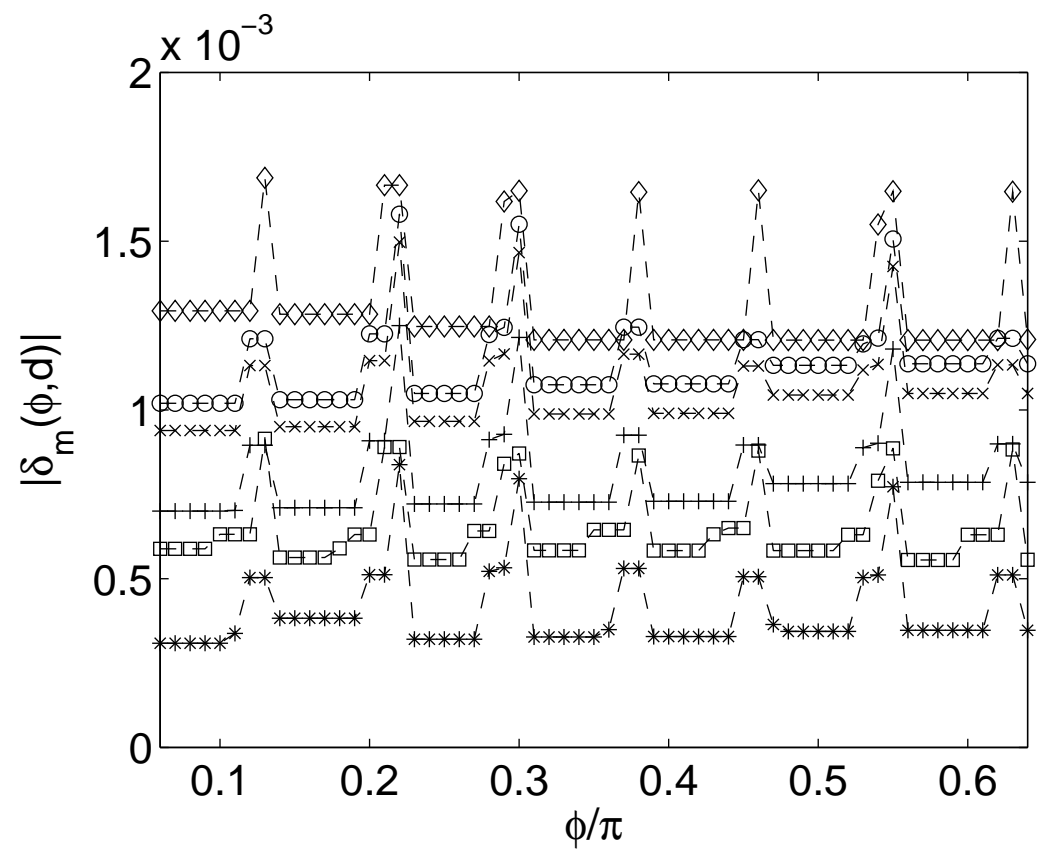

(b)

Figure 6.16. Example C: the maximum magnitude errors vs. $d$ and $\phi$, of the VBFD filters designed by (a) the Proposed ${ }_{1}$ and (b) the Proposed ${ }_{2}$, respectively. 


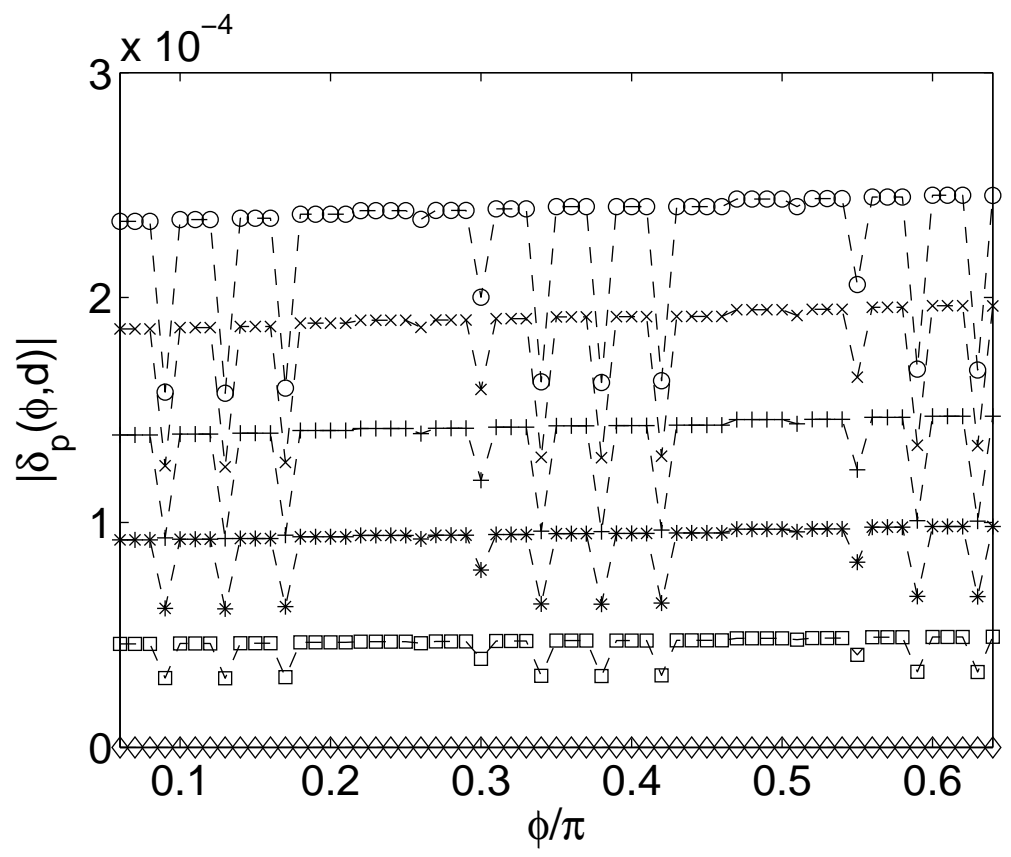

(a)

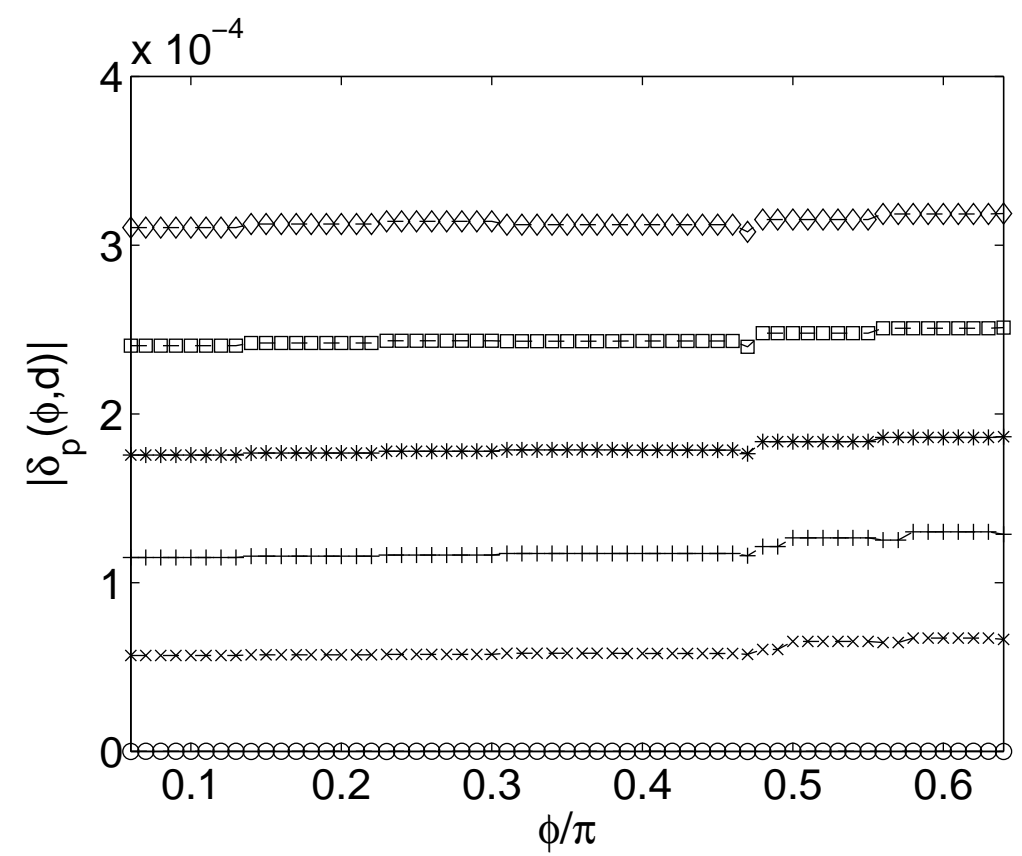

(b)

Figure 6.17. Example C: the maximum phase errors vs. $d$ and $\phi$, of the VBFD filters designed by (a) the Proposed 1 and (b) the Proposed ${ }_{2}$, respectively. 


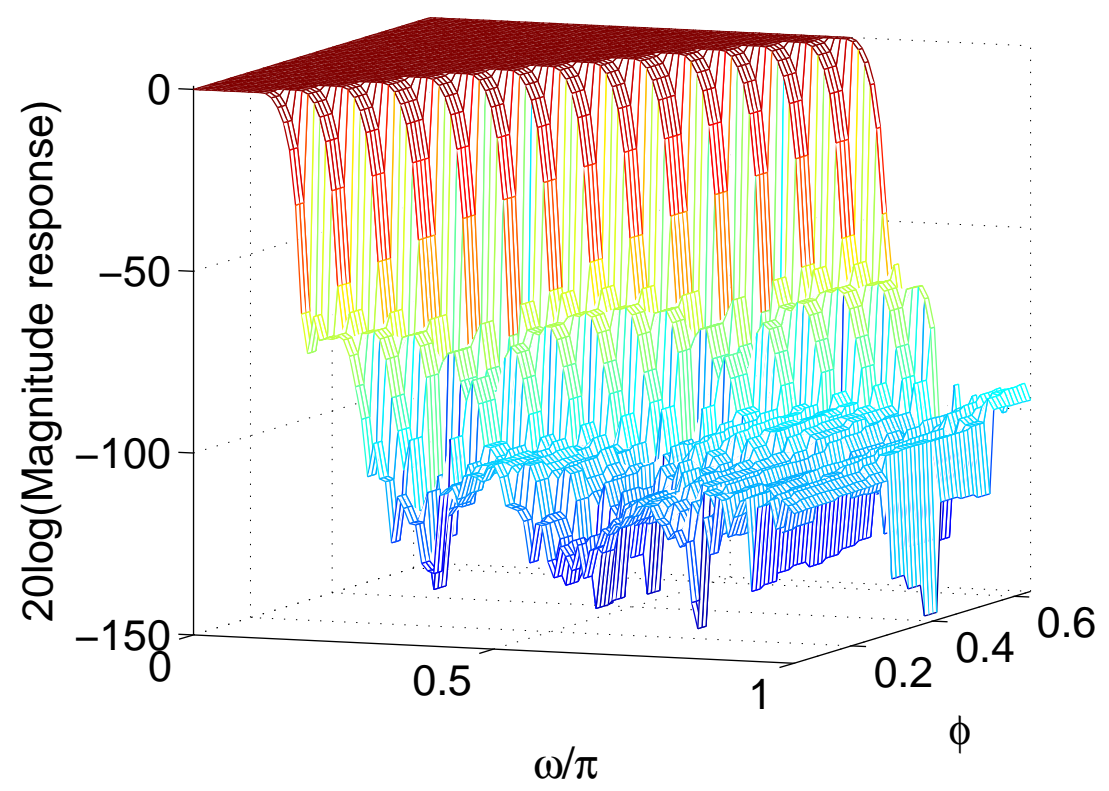

(a)

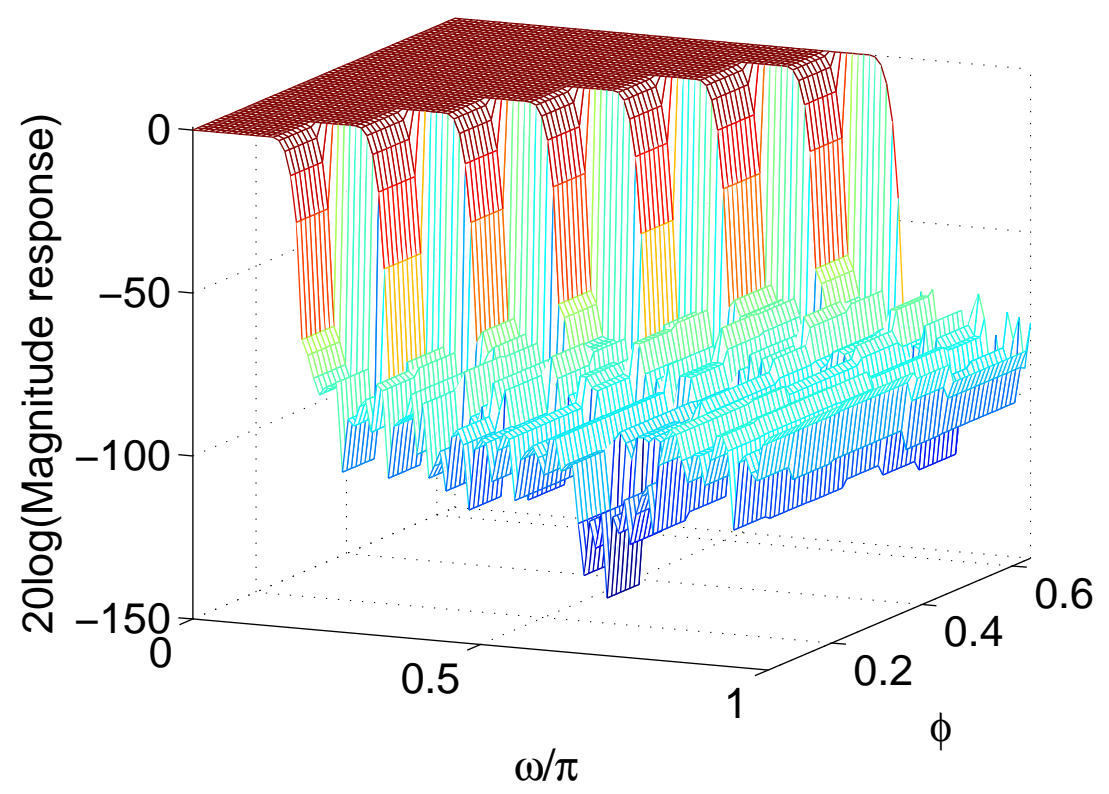

(b)

Figure 6.18. Example C: the magnitude responses of the VBFD filters with passband edge $\phi$ varying from $0.06 \pi$ to $0.64 \pi$ and fixed fractional delay $d= \pm 0.3$ using (a) the Proposed and (b) the Proposed 2 , respectively. 
It is noted that currently the two-dimensional Farrow structure cannot design the VBFD filters with such stringent specifications.

\subsection{Summary}

This chapter incorporates the variable bandedge characteristic into the VFD filter proposed in Chapter 5. Two approaches are proposed. The first approach directly combines the necessary bands and discard the undesired bands produced by the FFB to generate the VBFD, and the other one introduces shaping filters for the design of VBFD filters with sharper transition band. When the shaping filters are applicable, the transition width of the bands in the FFB can be relaxed, such that the FFB complexity can be reduced. Both approaches reduce the computational complexity at the price of more delay ele-

ments. Dependent on the specifications of the VBFD filters. the saving in the adders and constant multipliers varies in the range of $[20 \%, 80 \%]$ and $[40 \%, 90 \%]$, respectively. 
Table 6.2

COMPUTATIONAL COMPLEXITY AND IMPLEMENTATION COMPLEXITY OF EXAMPLE A

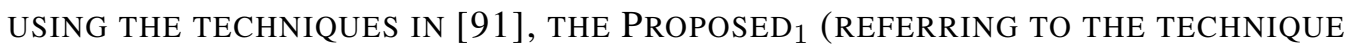
WITHOUT SHAPING FILTERS) AND THE PROPOSED 2 (REFERRING TO THE TECHNIQUE WITH SHAPING FILTERS).

\begin{tabular}{|c|c|c|c|c|c|}
\hline \multicolumn{3}{|c|}{ Technique } & Deng [91] & $\begin{array}{c}\text { Proposed }_{1} \\
\text { (saving to }[91] \text { ) }\end{array}$ & $\begin{array}{c}\text { Proposed }_{2} \\
\text { (saving to [91]) }\end{array}$ \\
\hline \multicolumn{3}{|c|}{$R$} & - & 4 & 3 \\
\hline \multicolumn{3}{|c|}{$K_{r} ; Q$} & - & $8,10,6,6$ & $8,14,6 ; 14$ \\
\hline \multirow{4}{*}{ Computation } & \multicolumn{2}{|c|}{ Additions } & 874 & 242 & 155 \\
\hline & \multirow{2}{*}{ Multiplications } & Constant & 450 & $\begin{array}{c}71 \\
84.22 \%\end{array}$ & $\begin{array}{c}56 \\
87.56 \%\end{array}$ \\
\hline & & General & 24 & $\begin{array}{c}26 \\
-8.33 \%\end{array}$ & $\begin{array}{c}17 \\
29.17 \%\end{array}$ \\
\hline & \multicolumn{2}{|c|}{ Group Delay } & 17 & $\begin{array}{c}61 \\
-258.82 \% \\
\end{array}$ & $\begin{array}{c}61 \\
-258.82 \% \\
\end{array}$ \\
\hline \multirow{4}{*}{ Implementation } & \multicolumn{2}{|c|}{ Adders } & 874 & $\begin{array}{c}242 \\
72.31 \%\end{array}$ & $\begin{array}{c}207 \\
76.32 \%\end{array}$ \\
\hline & \multirow{2}{*}{ Multipliers } & Constant & 450 & $\begin{array}{c}71 \\
84.22 \%\end{array}$ & $\begin{array}{c}75 \\
83.33 \%\end{array}$ \\
\hline & & General & 24 & $\begin{array}{c}26 \\
-8.33 \%\end{array}$ & $\begin{array}{c}17 \\
29.17 \%\end{array}$ \\
\hline & \multicolumn{2}{|c|}{ Delay Units } & 34 & $\begin{array}{c}328 \\
-864.71 \%\end{array}$ & $\begin{array}{c}516 \\
-1417.65 \%\end{array}$ \\
\hline
\end{tabular}


Table 6.3

COMPUTATIONAL COMPLEXITY AND IMPLEMENTATION COMPLEXITY OF EXAMPLE B USING THE TECHNIQUES IN [92] AND THE PROPOSED 1 (REFERRING TO THE TECHNIQUE WITHOUT SHAPING FILTERS).

\begin{tabular}{|c|c|c|c|c|}
\hline \multicolumn{3}{|c|}{ Technique } & Johansson [92] & $\begin{array}{c}\text { Proposed }_{1} \\
\text { (saving to [92]) }\end{array}$ \\
\hline \multicolumn{3}{|c|}{$R$} & - & 4 \\
\hline \multicolumn{3}{|c|}{$K_{r} ; Q$} & $-;-$ & $10,18,10,6 ;-$ \\
\hline \multirow{4}{*}{ Computation } & \multicolumn{2}{|c|}{ Additions } & 384 & $\begin{array}{c}283 \\
(26.30 \%) \\
\end{array}$ \\
\hline & \multirow{2}{*}{ Multiplications } & Constant & 174 & $\begin{array}{c}91 \\
(47.70 \%)\end{array}$ \\
\hline & & General & 23 & $\begin{array}{c}26 \\
(-13.04 \%) \\
\end{array}$ \\
\hline & \multicolumn{2}{|c|}{ Group Delay } & 21 & $\begin{array}{c}89 \\
(-323.81 \%) \\
\end{array}$ \\
\hline \multirow{4}{*}{ Implementation } & \multicolumn{2}{|c|}{ Adders } & 384 & $\begin{array}{c}283 \\
(26.30 \%)\end{array}$ \\
\hline & \multirow{2}{*}{ Multipliers } & Constant & 174 & $\begin{array}{c}91 \\
(47.70 \%) \\
\end{array}$ \\
\hline & & General & 23 & $\begin{array}{c}26 \\
(-13.04 \%)\end{array}$ \\
\hline & \multicolumn{2}{|c|}{ Delay Units } & 56 & $\begin{array}{c}488 \\
(-771.43 \%)\end{array}$ \\
\hline
\end{tabular}


Table 6.4

COMPUTATIONAL COMPLEXITY AND IMPLEMENTATION COMPLEXITY OF EXAMPLE C USING THE PROPOSED 1 (REFERRING TO TECHNIQUE WITHOUT SHAPING FILTERS) AND PROPOSED $_{2}$ (REFERRING TO THE TECHNIQUE WITH SHAPING FILTERS) TECHNIQUES.

\begin{tabular}{|c|c|c|c|c|}
\hline \multicolumn{3}{|c|}{ Technique } & Proposed $_{1}$ & Proposed $_{2}$ \\
\hline \multicolumn{3}{|c|}{$R$} & 5 & 4 \\
\hline \multicolumn{3}{|c|}{$K_{r} ; Q$} & $10,18,14,10,6 ;-$ & $14,22,10,6 ; 14$ \\
\hline \multirow{4}{*}{ Computation } & \multicolumn{2}{|c|}{ Additions } & 641 & 340 \\
\hline & \multirow{2}{*}{ Multiplications } & Constant & 178 & 112 \\
\hline & & General & 56 & 32 \\
\hline & \multicolumn{2}{|c|}{ Group Delay } & 193 & 169 \\
\hline \multirow{4}{*}{ Implementation } & \multicolumn{2}{|c|}{ Adders } & 641 & 392 \\
\hline & \multirow{2}{*}{ Multipliers } & Constant & 178 & 131 \\
\hline & & General & 56 & 32 \\
\hline & \multicolumn{2}{|c|}{ Delay Units } & 1312 & 2416 \\
\hline
\end{tabular}




\section{Chapter 7}

\section{Conclusion and Future Work}

In this chapter, the research work is concluded and some potential future work is briefly discussed.

\subsection{Research Work Conclusion}

In this thesis, both the closed-form and numerical optimization of VFD filters are investigated.

In the closed-form design, the coefficient dependance of the Farrow structure based Lagrange interpolation is studied. It is discovered that the coefficients of the subfilters can be represented as scaled versions of their respective adjacent subfilter coefficients. Meanwhile, scaled by a common integer factor, all the subfilter coefficients can be transformed to integers and thus no quantization error is incurred for finite word implementation. Based on these discoveries, an efficient implementation structure is proposed to achieve the lower computational complexity and smaller frequency response errors than the existing techniques.

On the other hand, in the numerical optimization approach, the design criteria of VFD filters must be first identified. Among various approaches, VFD filters are mainly used for the estimation of time domain instantaneous delayed sample. This thesis proves that the frequency response error, determined by the magnitude error and phase error, is the 
criteria that determines the time domain sample errors. This claim is also verified by time domain simulations for different types of signals.

Besides the investigation on the design criteria, a filter bank based technique for the design of VFD filters is proposed. In this approach, the input signal spectrum is split by a mixed-radix FFB into several bands. By shifting each band a fractional delay related phase, the VFD filter is realized by combining all bands. The optimization of the FFB is discussed in detail. The further improved optimization of the FFB is also proposed.

Based on the mixed-radix FFB approach, two approaches incorporating variable bandedge for the design of VBFD filters are proposed. One is to directly combine the necessary bands and discard the remaining, while the other introduces shaping filters to shape the last retained band. The latter approach may relax the design of the FFB to reduce the complexity at the cost of extra delayed units. The optimization of the FFB for the VBFD filters and the implementation structure of the technique are presented in detail.

\subsection{Future Work Briefing}

Future works may be developed from the following aspects.

1. Applying the FFB based VFD technique into the FFB based tunable notch filters and simultaneously tunable fractional delay and notch filters.

In audio signal processing, tonal equalization and filtering are the most fundamental processes in audio recording. Besides the VFD filters, tunable notch filters are also widely used in this domain. The proposed FFB based VFD technique is applicable in the design of tunable notch filters. The implementation structure using the FFB is shown in Figure 7.1.

In Figure 7.1, the switches $S_{k}\left(k=0, \ldots, \frac{N}{2}\right)$ are all connected except $S_{n}$ when the desired notch frequency falls into the range of band $n$. It can be found that the notch frequencies are the center frequencies of the bands. 


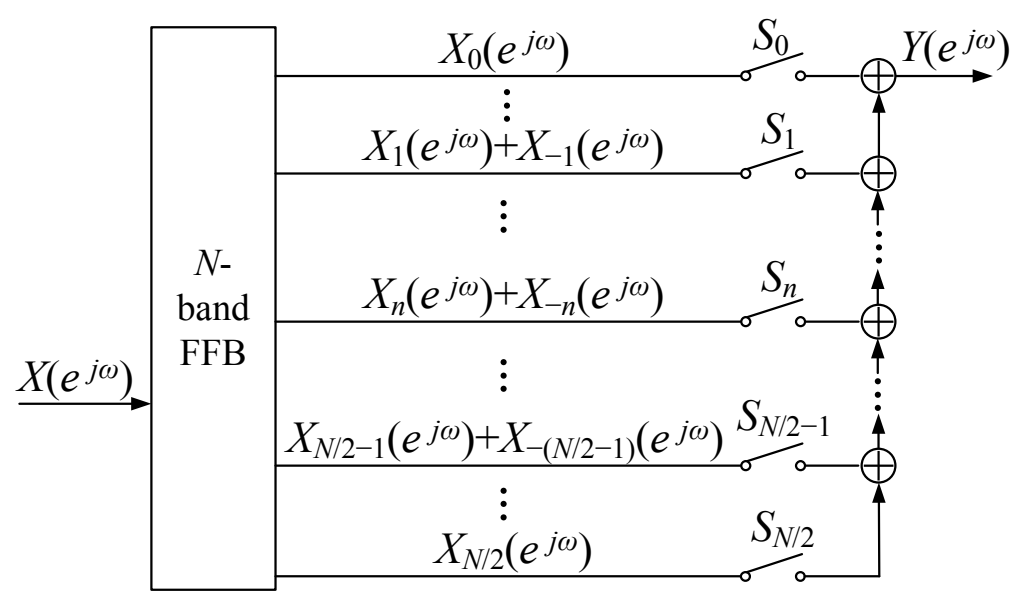

Figure 7.1. The implementation of tunable notch filters based on the FFB technique.

For some applications which requires both tunable fractional delay and notch frequency, simultaneously tunable fractional delay and notch filters can be designed using the proposed FFB technique similarly as shown in Figure 7.2.

This implementation structure avoids transients, compared with the cascade of a VFD filter and a tunable notch filter. Different from VFD filters, which are regarded as all pass filter and VBFD filters which are regarded as low pass filters, both tunable notch filter and simultaneously tunable fractional delay and notch filter require precise stopband edges, and hence the over design of the FFB is not helpful and the challenge is to find a solution to this issue.

2. Applying the FFB based VFD technique into the design of VFD filters with different fractional delays for different frequency ranges.

In some applications, the desired fractional delay may not be consistent with time, but with frequency. Hence the design of VFD filters with different fractional delays for different frequency ranges is useful. In Figure 5.7 of Chapter 5, the VFD is synthesized by applying phase shift $e^{-j 2 k d \pi / N}$ to band $N$ and hence the resultant fractional delay is $d$ for the whole frequency range. Beneficial from the proposed FFB based VFD technique, different fractional delays in different frequency ranges can be simply realized by 


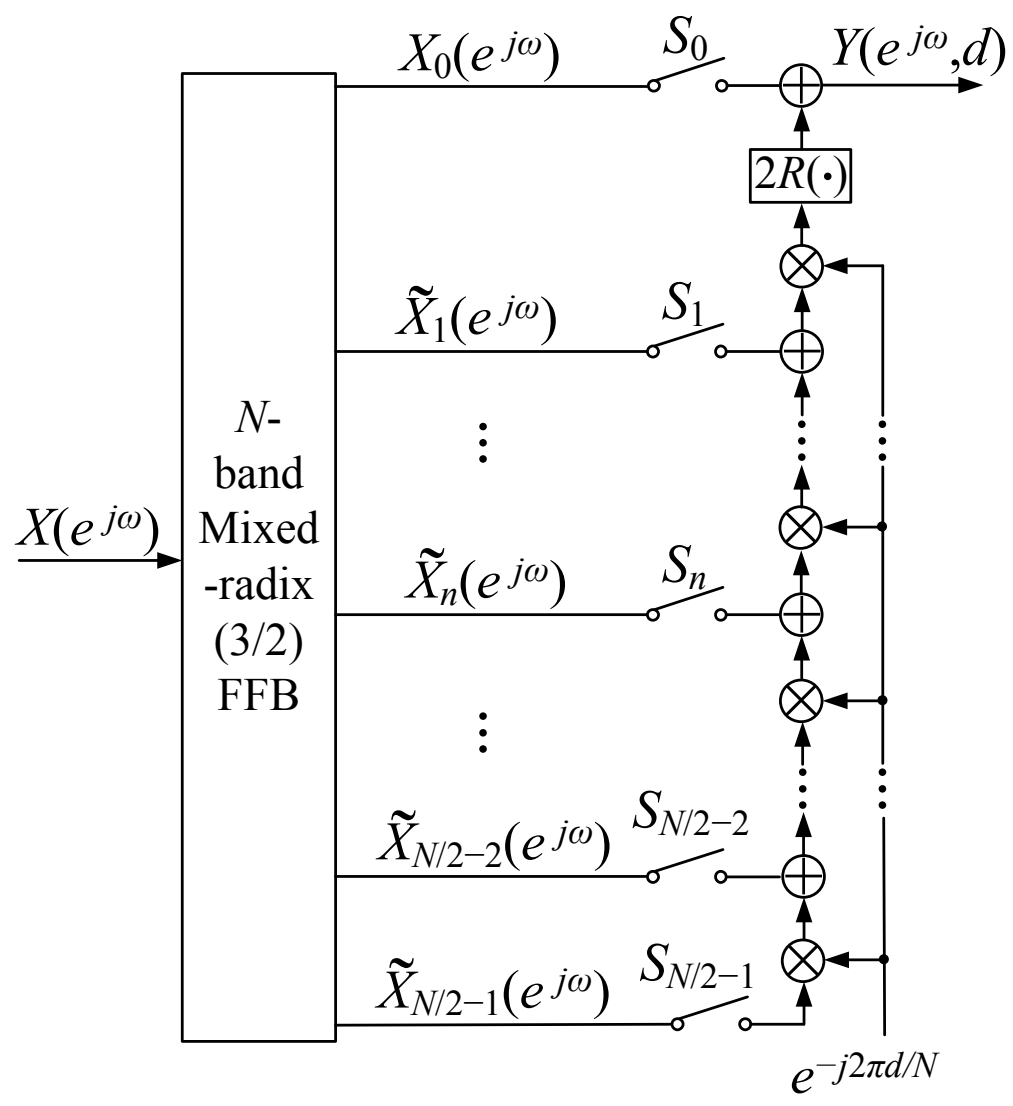

Figure 7.2. The implementation of simultaneously tunable fractional delay and notch filters based on the FFB technique.

applying different $d$ for phase shifts of different bands.

A design example is given where a 4-stage FFB with $K 0=6, K_{1}=22, K_{2}=14$ and $K_{3}=10$ is in use. By applying $d=0.1,0.2,0.3$ to the phase shifts of bands $\pm 1, \pm 2, \pm 3, \pm 4$, bands $\pm 5, \pm 6, \pm 7, \pm 8$ and bands $\pm 9, \pm 10, \pm 11$, the magnitude response and phase delay are illustrated in Figures 7.3(a) and (b), respectively.

It can be found the magnitude still remains almost unity for the passband from Figure 7.3(a), and the fractional delay values has adjusted to $0.1,0.2$ and 0.3 for different frequency ranges as shown in Figure 7.3(b). During the fractional delay value changes, there exist transition bands. Hence, to reduce the transition bands, finer FFB bands are desired.

3. Optimization of the VFD filters in discrete coefficient space for the purpose of 


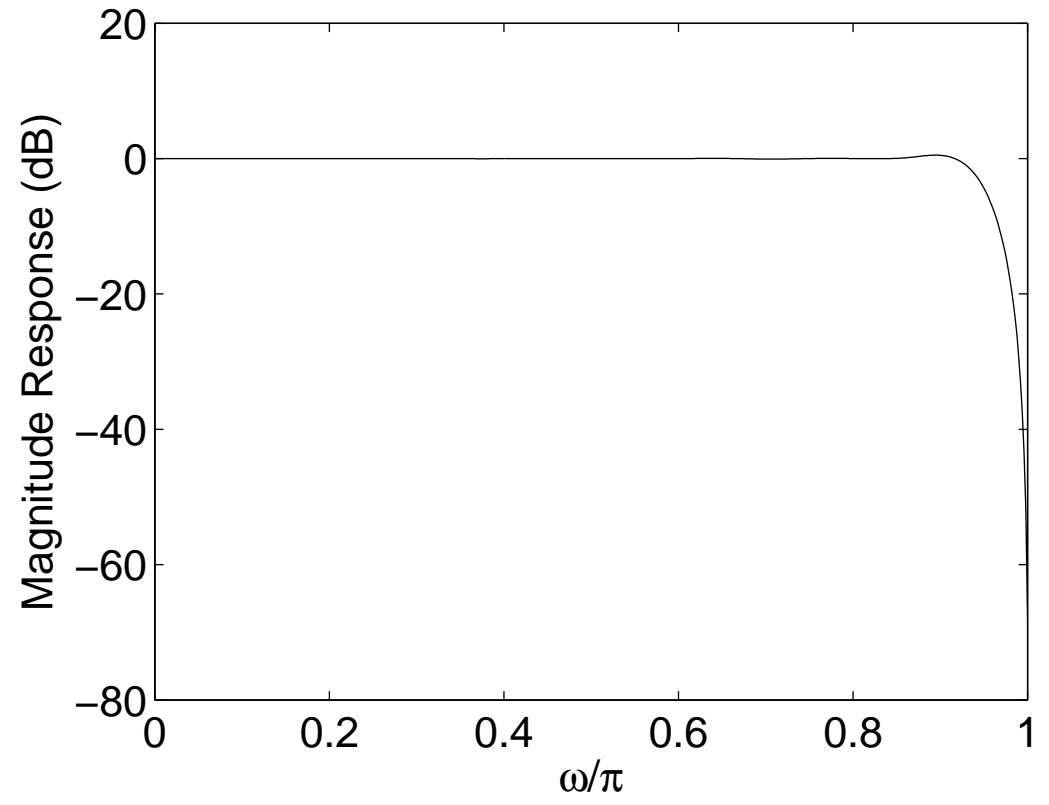

(a)

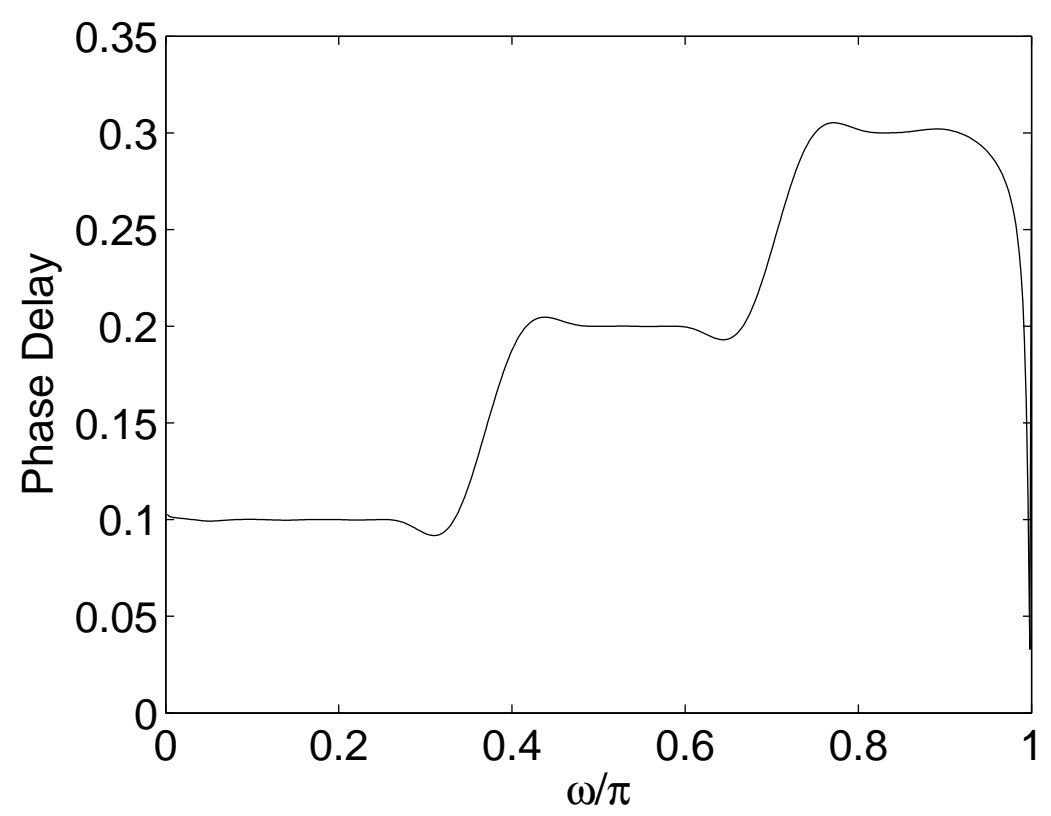

(b)

Figure 7.3. (a) The magnitude response and (b) the phase delay of the FFB based VFD filters. 
digital implementations.

For practical applications, the coefficients of digital filters are implemented in the discrete space. The coefficient multipliers with finite length can be implemented by adders and hard-wired shifts, hence multiplierless VFD filters can be resulted. A recently technique of optimization of discrete-value linear phase FIR filters [137] can be applied in the proposed FFB based VFD and VBFD approach.

4. New structure for the filter bank based VFD filters.

The proposed filter bank based technique suffers from long group delays. New structures employing filter bank without long delay, such as cosine modulated filter banks [138], may be considered.

5. Besides the application of the estimation of the time domain instantaneous delayed samples like time delay estimation [113], sampling rate conversion [102, 124-127], the VFD filters may also find their applications in image processing and biomedical signal processing. In the meanwhile, investigating the design criteria for these applications are meaningful.

6. Study how to design stable IIR variable filters with simultaneously adjustable bandedge and fractional delay.

With the recent design techniques of IIR VFD filters [84, 139], the FIR filter bank in the proposed FFB based VFD and VBFD filters may be replaced with IIR filters. 


\section{Bibliography}

[1] R. E. Crochiere and L. R. Rabiner, Multirate Digital Signal Processing. Englewood Cliffs, New Jersey: Prentice-Hall, 1983.

[2] S. Haykin, Adaptive Filter Theory. Englewood Cliffs, New Jersey: Prentice-Hall, 1986.

[3] P. P. Vaidyanathan, Multirate Systems and Filter Banks. Englewood Cliffs, New Jersey: Prentice-Hall, 1993.

[4] T. Saramäki, "Finite impluse response filter design," in Handbook for digital signal processing, S. K. Mitra and J. F. Kaiser, Eds. New York: John Wiley and Sons, 1993, ch. 4.

[5] S. K. Mitra, Digital Signal Processing, A Computer-Based Approach. McGrawHill, 1998, ch. 10.

[6] F. A. Marvasti, Nonuniform Sampling. Springer, 2001.

[7] T. W. Parks and C. S. Burrus, Digital filter design. Wiley, 1987.

[8] P. P. Vaidyanathan, "Multirate digital filters, filter banks, polyphase networks, and applications: A tutorial," Proc. IEEE, vol. 78, pp. 56-93, Jan. 1990.

[9] T. Saramäki and S. K. Mitra, "Multiple branch FIR filters for sampling rate conversion," in Proc. IEEE ISCAS'92, 1992, pp. 1007-1010. 
[10] W. Schuessler and W. Winkelnkemper, "Variable digital filters," Arch. Electr. Ubertr., vol. 24, pp. 524-525, 1970.

[11] A. V. Oppenheim, W. F. G. Mechlenbräuker, and R. M. Mersereau, "Variable cutoff linear phase digital filters," IEEE Trans. Circuits Syst., vol. 23, pp. 199-203, Apr. 1976.

[12] R. E. Crochiere and R. L. Rabiner, "On the properties of frequency transformations for variable cutoff linear phase digital filters," IEEE Trans. Circuits Syst., vol. 23, pp. 684-686, Nov. 1976.

[13] S. C. D. Roy and S. S. Ahuja, "Frequency transformation for linear-phase variablecutoff digital filters," IEEE Trans. Circuits Syst., vol. 26, pp. 73-75, Jan. 1979.

[14] S. S. Ahuja and S. C. D. Roy, "Linear phase variable digital bandpass filters," in Proc. IEEE, vol. 67, Jan. 1979, pp. 173-174.

[15] S. N. Hazra, "Linear phase bandpass digital filters with variable cutoff frequencies," IEEE Trans. Circuits Syst., vol. 31, pp. 661-663, 1984.

[16] Y. N. P. Jarske and S. K. Mitra, "A simple approach to the design of linear phase FIR digital filters with variable characteristics," Signal Processing (Elsevier), vol. 14, pp. 313-326, 1988.

[17] P. Jarske, S. C.-H. Chang, and Y. Neuvo, "Variable linear phase FIR filters," in Proc. IEEE ICASSP'88, 1988, pp. 1463-1466.

[18] R. Zarour and M. M. Fahmy, "A design technique for variable digital filters," IEEE Trans. Circuits Syst. I, vol. 36, pp. 1473-1478, Nov. 1989.

[19] G. Stoyanov and M. Kawamata, "Variable digital filters," J. Signal Processing, vol. 1, pp. 275-289, Apr. 1997. 
[20] T.-B. Deng, "Decomposition-based design of linear phase variable digital filters," IEEE Trans. Circuits Syst. I, vol. 46, pp. 399-402, Mar. 1999.

[21] _ _ "Weighted least-squares method for designing arbitrarily variable 1-D FIR digital filters," Signal Processing (Elsevier), vol. 4, pp. 597-613, 2000.

[22] — - "Design of linear-phase variable 2-D digital filters using matrix-array decomposition," IEEE Trans. Circuits Syst. II, vol. 50, pp. 267-277, 2003.

[23] H. Johansson and P. Löwenborg, "On linear-phase FIR filters with variable bandwidth,” IEEE Trans. Circuits Syst. II, vol. 51, pp. 181-184, 2004.

[24] S. C. Chan, C. K. S. Pun, and K. L. Ho, "A new method for designing FIR filters with variable characteristics," IEEE Signal Processing Lett., vol. 11, pp. 274-277, Feb. 2004.

[25] K. M. Tsui, K. S. Yeung, and S. C. Chan, "On the minimax design of passband linear-phase variable digital filters using semidefinite programming," IEEE Signal Processing Lett., vol. 11, pp. 867-870, Nov. 2004.

[26] H. Johansson and P. Löwenborg, "On linear-phase FIR filters with variable bandwidth," IEEE Trans. Circuits Syst. II, vol. 51, pp. 181-184, 2004.

[27] P. Löwenborg and H. Johansson, "Minimax design of adjustable-bandwidth linearphase FIR filters," IEEE Trans. Circuits Syst. I, vol. 53, pp. 431-439, Feb. 2006.

[28] J. Yli-Kaakinen and T. Saramäki, "Efficient recursive digital filters with variable magnitude characteristics," in Proc. IEEE NORSIG'06, June 2006, pp. 30-33.

[29] S. Kidambi, "An efficient closed-form approach to the design of linear-phase FIR digital filters with variable-bandwidth characteristics," Signal Processing (Elsevier), vol. 86, pp. 1656-1669, 2006. 
[30] T.-B. Deng, "Weighted least-squares method for designing arbitrarily variable 1-D FIR digital filters," Signal Processing (Elsevier), vol. 4, pp. 597-613, 2000.

[31] Y. J. Yu, Y. C. Lim, and D. Shi, "Low-complexity design of variable bandedge linear phase FIR filters with sharp transition band," IEEE Trans. Signal Processing, vol. 57, pp. 1328-1338, Apr. 2009.

[32] J. J. Shyu, S. C. Pei, and Y. D. Huang, “3-D FIR cone-shaped filter design by a nest of McClellan transformations and its vaariable design," IEEE Trans. Circuits Syst. I, vol. 57, pp. 1697-1707, 2010.

[33] V. Välimäki and A. Haghparast, "Fractional delay filter design based on truncated Lagrange interpolation,” IEEE Signal Processing Lett., vol. 14, pp. 816-819, Nov. 2007.

[34] T.-B. Deng, "Coefficient-symmetries for implementing arbitrary-order Lagrangetype variable fractional-delay digital filters," IEEE Trans. Signal Processing, vol. 55, pp. 4078-4090, Aug. 2007.

[35] — - "Robust structure transformation for causal Lagrange-type variable fractional-delay filters," IEEE Trans. Circuits Syst. I, vol. 56, pp. 1681-1688, Aug. 2009.

[36] W. J. Xu and Y. J. Yu, "Polynomial implementation structure for Lagrange-type variable fractional delay," in Proc. IEEE ISCAS'2010, May 2010, pp. 733-736.

[37] C.-C. Tseng and S.-L. Lee, "Design of fractional delay filter using discrete fourier transform interpolation method," Signal Processing, vol. 90, pp. 1313-1322, Apr. 2010.

[38] V. Välimäki, “A new filter implementation strategy for Lagrange interpolation,” in Proc. IEEE ISCAS'95, vol. 1, 1995, pp. 361-364. 
[39] A. Haghparast and V. Välimäki, "A computationally efficient coefficient update technique for Lagrange fractional delay filters," in Proc. IEEE ICASSP'2008, Mar. 2008, pp. 3737-3740.

[40] E. Hermanowicz, "Explicit formulas for weighting coefficients of maximally flat tunable FIR delayers," Electronics Lett., vol. 28, pp. 1936-1937, Sept. 1992.

[41] A. G. Dempster and N. P. Murphy, "Lagrange interpolator filters and binomial windows," Signal Processing (Elsevier), vol. 76, pp. 81-91, Jan. 1999.

[42] J. Yekta and M. Mahdi, "Half-band FIR fractional delay filters with closed-form coefficient formulas and modular implementation based on lagrange interpolators," Signal Processing (Elsevier), vol. 88, pp. 2913-2916, Dec. 2008.

[43] _ _ "A frequency domain proof for the equivalence of the maximally flat FIR fractional delay filter and the lagrange interpolator," Digital Signal Processing (Elsevier), vol. 21, pp. 13-16, Jan. 2011.

[44] C.-C. Tseng, "Design of variable fractional delay FIR filter using differentiator bank," in Proc. IEEE ISCAS'02, vol. 4, May 2002, pp. 421-424.

[45] C. K. S. Pun, S. C. Chan, K. S. Yeung, and K. L. Ho, "On the design and implementation of FIR and IIR digital filters with variable frequency characteristics," IEEE Trans. Circuits Syst. II, vol. 49, pp. 689-703, Nov. 2002.

[46] J. Yli-Kaakinen and T. Saramäki, "Mulitplication-free polynomial-based FIR filters with an adjustable fractional delay," in Circuits Syst. Signal Process, vol. 25, 2006, pp. 265-294.

[47] S.-C. Pei and H.-S. Lin, “Tunable FIR and IIR fractional-delay filter design and structure based on complex cepstrum," IEEE Trans. Circuits Syst. I, vol. 56, pp. 2195-2206, Oct. 2009. 
[48] C. W. Farrrow, "A continuously variable digital delay element," in Proc. IEEE ISCAS'88, vol. 3, 1988, pp. 2641-2645.

[49] T. I. Laakso, V. Välimäki, M. Karjalainen, and U. K. Laine, "Splitting the unit delay: Tools for fractional delay filter design," IEEE Trans. Signal Processing, vol. 13, pp. 30-60, Jan. 1996.

[50] J. Vesma and T. Saramäki, "Interpolation filters with arbitrary frequency response for all-digital receivers," in Proc. IEEE ISCAS'96, vol. 2, 1996, pp. 568-571.

[51] — - "Optimization and efficient implementation of FIR filters with adjustable fractional delay," in Proc. IEEE ISCAS'97, vol. 4, 1997, pp. 2256-2259.

[52] _ _ "Design and properties of polynomial-based fractional delay filters," in Proc. IEEE ISCAS'2000, vol. 1, 2000, pp. 104-107.

[53] H. Johansson and P. Löwenborg, "On the design of adjustable fractional delay FIR filters," IEEE Trans. Circuits Syst. II, vol. 50, pp. 164-169, 2003.

[54] J. Yli-Kaakinen and T. Saramäki, "A simplified structure for FIR filters with an adjustable fractional delay," in Proc. IEEE ISCAS'2007, vol. 3, May 2007, pp. $3439-3442$.

[55] J.-J. Shyu, S.-C. Pei, C.-H. Chan, and Y.-D. Huang, "Minimax design of variable fractional-delay FIR digital filters by iterative weighted least-squares approach," IEEE Signal Processing Lett., vol. 15, pp. 693-696, 2008.

[56] T.-B. Deng, "Decoupling minimax design of low-complexity variable fractionaldelay FIR digital filters," IEEE Trans. Circuits Syst. I, vol. 58, pp. 2398-2408, Oct. 2011. 
[57] T.-B. Deng, S. Chivapreecha, and K. Dejhan, "Bi-minimax design of even-order variable fractional-delay FIR digital filters," IEEE Trans. Circuits Syst. I, vol. 59, pp. 1766-1774, Aug. 2012.

[58] T.-B. Deng and W. Qin, "Coefficient relation-based minimax design and lowcomplexity structure of variable fractional-delay digital filters," Signal Processing (Elsevier), vol. 93, pp. 923-932, 2013.

[59] A. Eghbali, H. Johansson, and T. Saramäki, "A method for the design of farrowstructure based variable fractional-delay FIR filters," Signal Processing (Elsevier), vol. 93, pp. 1314-1318, May 2013.

[60] T.-B. Deng, "Design of variable FIR digital filters with arbitrary frequency responses," in Tech. Rep. IEICE, vol. DSP96-153, Mar. 1997, pp. 9-15.

[61] A. Tarczynski, G. D. Cain, E. Hermanowicz, and M. Rojewski, "WLS design of variable frequency response FIR filters,' in Proc. IEEE ISCAS'97, vol. 4, 1997, pp. 2244-2247.

[62] W.-S. Lu and T.-B. Deng, "An improved Weighted Least-Squares design for variable fractional delay FIR filters," IEEE Trans. Circuits Syst. II, vol. 46, pp. 10351040, Aug. 1999.

[63] C.-C. Tseng, "Design of variable fractional delay FIR filter using symmetry," in Proc. IEEE ISCAS'2004, vol. 4, Vancouver, Canada, May 2004, pp. 477-480.

[64] T.-B. Deng and Y. Nakagawa, "SVD-based design and new stuctures for variable fractional-delay digital filters," IEEE Trans. Signal Processing, vol. 52, pp. 25132527, Sept. 2004. 
[65] T.-B. Deng and Y. Lian, "Weighted-least-squares design of variable fractionaldelay FIR filters using coefficient symmetry," IEEE Trans. Signal Processing, vol. 54, pp. 3023-3038, Aug. 2006.

[66] T.-B. Deng, "Symmetric structures for odd-order maximally flat and weightedleast-squares variable fractional-delay filters," IEEE Trans. Circuits Syst. I, vol. 54, pp. 2718-2732, Dec. 2007.

[67] J.-J. Shyu and S.-C. Pei, "A generalized approach to the design of variable fractional-delay FIR digital filters," Signal Processing (Elsevier), vol. 88, pp. 1428-1435, 2008.

[68] J. Selva, "An efficient structure for the design of variable fractional delay filters based on the windowing method," IEEE Trans. Signal Processing, vol. 56, pp. 3770-3775, Aug. 2008.

[69] H. K. Kwan and A. Jiang, "FIR, Allpass, and IIR variable fractional delay digital filter design,” IEEE Trans. Circuits Syst. I, vol. 56, pp. 2064-2074, Sept. 2009.

[70] T.-B. Deng, "Hybrid structure for low-complexity variable fractional-delay FIR filters," IEEE Trans. Circuits Syst. I, vol. 57, pp. 897-910, Apr. 2010.

[71] J. J. Shyu, S. C. Pei, C. H. Chan, Y. D. Huang, and S. H. Lin, "A new criterion for the design of variable fractional-delay FIR digital filters," IEEE Trans. Circuits Syst. I, vol. 57, pp. 368-377, Feb. 2010.

[72] C.-C. Tseng and S.-L. Lee, "Efficient design and implementation of variable fractional delay filter using differentiators," IEEE Trans. Circuits Syst. I, vol. 58, pp. 1311-1322, 2011.

[73] V. Välimäki, "Simple design of fractional delay allpass filters," in Proc. EUSIPCO ISCAS'97, Sept. 2000, pp. 1881-1884. 
[74] M. Makundi, V. Välimäki, and T. I. Laakso, "Closed-form design of tunable fractional-delay allpass filter structure," in Proc. IEEE ISCAS'01, vol. 4, May 2001, pp. 434-437.

[75] S. S. Kidami, "Closed-form approach to design of allpass digital filters using cepstrum coefficients," Electronics Letters, vol. 40, pp. 720-721, 2004.

[76] J. Yekta and M. Mahdi, "Fast design of relatively wide band fractional delay allpass filters," Signal Processing (Elsevier), vol. 88, pp. 612-623, Mar. 2008.

[77] S.-C. Pei, H.-S. Lin, and P.-H. Wang, "Design of allpass fractional delay filter and fractional Hilbert transformer using closed-form of cepstral coefficients," in Proc. IEEE ISCAS’2007, May 2007, pp. 3443-3446.

[78] K. M. Tsui, S. C. Chan, and H. K. Kwan, "A new design method for designing causal stable IIR variable fractional delay digital filters," IEEE Trans. Circuits Syst. II, vol. 54, pp. 999-1003, Nov. 2007.

[79] J. Yli-Kaakinen and T. Saramäki, “An algorithm for the optimization of adjustable fractional-delay all-pass filters," in Proc. IEEE ISCAS'2004, vol. 3, Vancouver, Canada, May 2004, pp. 153-156.

[80] J.-J. Shyu, S.-C. Pei, and C.-H. Chan, "Minimax phase error design of allpass variable fractional-delay digital filters by iterative weighted least-squares method," Signal Processing (Elsevier), vol. 89, pp. 1774-1781, Sept. 2009.

[81] C.-C. Tseng, "Design of variable fractional delay allpass filter using weighted least squares method," in Proc. IEEE ISCAS'02, vol. 5, May 2002, pp. 713-716.

[82] _ _ "Design of 1-D and 2-D variable fractional delay allpass filters using weight leasted-squares method," IEEE Trans. Circuits Syst. I, vol. 49, pp. 1413-1422, Oct. 2002. 
[83] T.-B. Deng, "Noniterative WLS design of allpass variable fractional-delay digital filters," IEEE Trans. Circuits Syst. I, vol. 53, pp. 358-371, Feb. 2006.

[84] H. Zhao and H. K. Kwan, “Design of 1-D stable fractional delay IIR filters," IEEE Trans. Circuits Syst. II, vol. 54, pp. 86-90, Jan. 2007.

[85] L. W. Rong, L. Caccetta, and V. Rehbock, “Optimal design of All-Pass variable fractional-delay digital filters," IEEE Trans. Circuits Syst. I, vol. 55, pp. 12481256, May 2008.

[86] T.-B. Deng, "Generalized WLS method for designing All-Pass variable fractionaldelay digital filters," IEEE Trans. Circuits Syst. I, vol. 56, pp. 2207-2220, Oct. 2009.

[87] H. K. Kwan and A. Jiang, "Low-order fixed denominator IIR VFD filter design," in Proc. IEEE ISCAS'09, May 2009, pp. 481-484.

[88] H. Johansson and E. Hermanowicz, "Two-rate based low-complexity variable fractional-delay FIR filter structures," IEEE Trans. Circuits Syst. I, vol. 60, pp. 136-149, Jan. 2013.

[89] T.-B. Deng, "Optimal design and parallel implementation of FIR filters with variable magnitude and fractional-delay responses," in Proc. IEEE ISCAS'02, vol. 1, May 2002, pp. 401-404.

[90] _ _ "Design and parallel implementation of FIR digital filters with simultaneously variable magnitude and non-integer phase-delay," IEEE Trans. Circuits Syst. II, vol. 50, pp. 243-250, May 2003.

[91] _ _ "Closed-form design and efficient implementation of variable digital filters with simultaneuously tunable magnitude and fractional-delay," IEEE Trans. Signal Processing, vol. 52, pp. 194-199, June 2004. 
[92] H. Johansson and A. Eghbali, "A realization of FIR filters with simultaneously variable bandwidth and fractional delay," in Proc. EUSIPCO'2012, Aug. 2012, pp. $2178-2182$.

[93] Y. J. Yu and W. J. Xu, "Mixed-radix fast filter bank approach for the design of variable digital filters with simultaneously tunable bandedge and fractional delay," IEEE Trans. Signal Processing, vol. 60, pp. 100-111, Jan. 2012.

[94] W. J. Xu, Y. J. Yu, and H. Johansson, “Improved filter bank approach for the design of variable bandedge and fractional delay filters," IEEE Trans. Circuits Syst. I, vol. PP, pp. 1-14, 2014.

[95] F. M. Gardner, "Interpolation in digital modems-part I," IEEE Trans. comm., vol. 41, pp. 502-508, Mar. 1993.

[96] L. Erup, F. M. Gardner, and F. A. Harris, "Interpolation in digital modems-part II: implementation and performance," IEEE Trans. comm., vol. 41, pp. 998-1008, 1993.

[97] T. A. Ramstad, "Sample-rate conversion by arbitrary ratios," in Proc. IEEE ICASSP'82, vol. 7, 1982, pp. 101-104.

[98] J. O. Smith and P. Gossett, "A flexible sampling-rate conversion method," in Proc. IEEE ICASSP'84, vol. 9, 1984, pp. 112-115.

[99] S. Cucchi, F. Desinin, and G. Sicuranza, "DSP implementation of arbitrary sampling frequency conversion for high quality sound application," in Proc. IEEE ICASSP'91, Mar. 1991, pp. 3609-3612.

[100] A. Tarczynski, W. Kozinski, and G. D. Cain, "Sampling rate conversion using fractional-sample delay," in Proc. IEEE ICASSP'94, 1994, pp. 285-288. 
[101] T. Hentschel and G. Fettweis, "Continuous-time digital filters for sample-rate conversion in reconfigurable radio terminals," in Proc. Eur. Wireless', Dresden, Germany, Sept. 2000, pp. 55-59.

[102] K. M. Tsui, S. C. Chan, and K. W. Tse, "Design of complex-valued variable digital filters and its application to the realization of arbitrary sampling rate conversions for complex signals," IEEE Trans. Circuits Syst. II, vol. 52, pp. 424-428, 2005.

[103] K. J. Cho, J. S. Park, B. K. Kim, J. G. Chung, and K. K. Parhi, "Design of a sample-rate converter from CD to DAT using fractional delay allpass filter," IEEE Trans. Circuits Syst. II, vol. 54, pp. 19-23, Jan. 2007.

[104] J. S. Marques, I. M. Trancoso, J. M. Tribolet, and L. B. Almeida, "Improved pitch predicition with fractional delays in CELP coding," in Proc. IEEE ICASSP'90, Mar. 1990, pp. 665-668.

[105] P. Kroon and B. S. Atal, "On the use of pitch predictors with high temporal resolution,” IEEE Trans. Signal Processing, vol. 39, pp. 733-735, Mar. 1991.

[106] Y. Medan, "Using super resolution pitch in waveform speech coders," in Proc. IEEE ICASSP'91, Mar. 1991, pp. 633-636.

[107] M. Karjalainer and U. K. Laine, "A model for real-time sound synthesis of guitar on a floating-point signal processor," in Proc. IEEE ICASSP'91, Mar. 1991, pp. $3653-3656$.

[108] V. Välimäki, M. Karjalainer, and U. K. Laine, "A real-time DSP implementation of a flute model," in Proc. IEEE ICASSP'92, Mar. 1992, pp. 249-252.

[109] — - "Real-time implementation techniques for a continuously variable digital delay in modeling musical instruments," in Proc. Int. Computer Music Conf. ICMC-92, Oct. 1992, pp. 140-141. 
[110] J. O. Smith and B. Friedlander, "Adaptive interpolated time-dealy estimation," IEEE Trans. Aerosp. Electron. Syst., vol. 21, pp. 180-199, Feb. 1985.

[111] S.-C. Pei and C.-C. Tseng, "A comb filter design using fractional-sample delay," IEEE Trans. Circuits Syst. II, vol. 45, pp. 649-653, May 1998.

[112] C.-C. Tseng, "Digital differentiator design using fractional delay filter and limit computation,” IEEE Trans. Circuits Syst. I, vol. 52, pp. 2248-2259, Oct. 2005.

[113] M. Olsson, H. Johansson, and P. Löwenborg, "Time-delay estimation using Farrow-based fractional-delay FIR filters: Approximation vs. estimation errors," in Proc. EUSIPCO’2006, 2006.

[114] C.-C. Tseng, "Improved design of digital fractional-order differentiators using fractional smaple delay," IEEE Trans. Circuits Syst. I, vol. 53, pp. 193-203, Jan. 2006.

[115] C.-C. Tseng and S.-L. Lee, "Digital IIR integrator design using Richardson extrapolation and fractional delay," IEEE Trans. Circuits Syst. I, vol. 55, pp. 2300-2309, Aug. 2008.

[116] C.-C. Tseng, "Series expansion design of variable fractional order integrator and differentiator using logarithm," Signal Processing, vol. 88, pp. 2278-2292, Sept. 2008.

[117] G. W. Medlin, "A novel design technique for tunable notch filters," in Proc. IEEE ISCAS'1990, 1990, pp. 471-474.

[118] K. E. Nelson and M. A. Soderstrand, "Full tunable digital heterodyne IIR filters," in Proc. IEEE ICASSP'1999, 1999, pp. 1141-1144. 
[119] A. Azam, D. Sasidaran, K. Nelson, G. Ford, L. Johnson, and M. Soderstrand, "Efficient pipelined tunable heterodyne notch filter implementation in FPGAs," in Proc. IEEE ISCAS'2000, 2000, pp. 373-376.

[120] Y. C. Lim, "Frequency-response masking approach for the synthesis of sharp linear phase digital filters," IEEE Trans. Circuits Syst., vol. 33, pp. 357-364, Apr. 1986.

[121] Y. C. Lim and B. Farhang-Boroujeny, "Fast filter bank (FFB)," IEEE Trans. Circuits Syst. II, vol. 39, pp. 316-318, May 1992.

[122] C. C. Tseng and S. L. Lee, "Design of fractional delay filter using discrete fourier transform interpolation method," Signal Processing (Elsevier), vol. 90, pp. 13131322, Apr. 2010.

[123] K. C. Pohlman, Principles of Digital Audio. Third Edition. New York: McGrawHill, 1995.

[124] T. A. Ramstad, "Digital methods for conversion between arbitrary sampling frequencies," IEEE Trans. Acoust., Speech, Signal Processing, vol. 32, pp. 577-591, Mar. 1984.

[125] K. Rajamani, Y.-S. Lai, and C. W. Farrow, "An efficient algorithm for sample rate conversion from CD to DAT," IEEE Signal Processing Lett., vol. 7, pp. 288-290, Oct. 2000.

[126] J.-S. Park, B.-K. Kim, J.-G. Chung, and K. K. Parhi, "An asynchronous samplerate converter from CD to DAT," in Proc. IEEE ICASSP'2003, vol. 2, 2003, pp. $509-512$.

[127] K.-J. Cho, J.-S. Park, B.-K. Kim, J.-G. Chung, and K. K. Parhi, "Design of a sample-rate converter from CD to DAT using fractional delay allpass filter," IEEE Trans. Circuits Syst. II, vol. 54, pp. 19-23, Jan. 2007. 
[128] H. Oru, "LU factorization of the Vandermonde matrix and its applications," $A p$ plied Mathematics Letters, vol. 56, pp. 982-987, Sept. 2007.

[129] I. G. Macdonald, "Symmetric Functions and Hall Polynomials." Oxford, England: Oxford Science Publications, 1999.

[130] A. G. Dempster and M. D. Macleod, "Constant integer multiplication using minimum adders," IEE Proc. Circuits, Devices, Syts., vol. 141, pp. 407-413, Oct. 1994.

[131] H. J. Pain, The Physics of Vibrations and Waves. John Wiley \& Sons, 2005.

[132] J. Selva, "Functionally weighted Lagrange interpolation of band-limited signals from nonuniform samples," IEEE Trans. Signal Processing, vol. 57, pp. 168-181, Jan. 2009.

[133] — - "Convolution-based trigonometric interpolation of band-limited signals," IEEE Trans. Signal Processing, vol. 56, pp. 5465-5477, Nov. 2008.

[134] A. W. M. van den Enden, "Efficiency in multirate and complex digital signal processing,” Ph.D. dissertation, Tech. Univ. Eindhoven, Eindhoven, The Netherlands, 2001.

[135] J. M. P. Langlois and D. Al-Khalili, "Phase to sinusoid amplitude conversion techniques for direct digital frequency synthesis," Proc. IEE Proc.-Circuits, Devices Syst., vol. 151, pp. 519-528, Dec. 2004.

[136] S. Boyd and L. Vandenberghe, Convex Optimization. Cambridge University Press, 2004.

[137] W. B. Ye and Y. J. Yu, "Single-stage and cascade design of high order multiplierless linear phase FIR filters using genetic algorithm," IEEE Trans. Circuits Syst. I, vol. 60, pp. 2987-2997, Nov. 2013. 
[138] R. Bregović and T. Saramäki, "Multiple branch FIR filters for sampling rate conversion," in Proc. IEEE ISCAS'2002, 2002, pp. 825-828.

[139] S. C. Pei, J. J. Shyu, Y. D. Huang, and C. H. Chan, "Improved methods for the design of variable fractional delay IIR digital filters," IEEE Trans. Circuits Syst. I, vol. 59, pp. 989-1000, May 2012. 